University of New Mexico

UNM Digital Repository

Nuclear Engineering ETDs

Engineering ETDs

$2-1-2012$

\title{
A novel method for modeling the neutron Time of Flight (nTOF) detector response in current mode to inertial confinement fusion experiments
}

Alan Nelson

Follow this and additional works at: https://digitalrepository.unm.edu/ne_etds

\section{Recommended Citation}

Nelson, Alan. "A novel method for modeling the neutron Time of Flight (nTOF) detector response in current mode to inertial confinement fusion experiments." (2012). https://digitalrepository.unm.edu/ne_etds/16

This Dissertation is brought to you for free and open access by the Engineering ETDs at UNM Digital Repository. It has been accepted for inclusion in Nuclear Engineering ETDs by an authorized administrator of UNM Digital Repository. For more information, please contact disc@unm.edu. 
Alan John Nelson

Candidate

Department of Chemical and Nuclear Engineering

Department

This dissertation is approved, and it is acceptable in quality and form for publication:

Approved by the Dissertation Committee:

Dr. Gary Cooper

, Chairperson

Dr. Carlos Ruiz

Dr. Anil Prinja

Dr. Mark Gilmore 
A NOVEL METHOD FOR MODELING THE NEUTRON TIME OF FLIGHT (nTOF) DETECTOR RESPONSE IN CURRENT MODE TO INERTIAL CONFINEMENT FUSION EXPERIMENTS

By

\section{ALAN JOHN NELSON}

B.S. NUCLEAR ENGINEERING

THE UNIVERSITY OF NEW MEXICO, 1988

M.S. NUCLEAR ENGINEERING

THE UNIVERISITY OF NEW MEXICO, 2003

DISSERTATION

Submitted in Partial Fulfillment of the

Requirements of the Degree of

Doctor of Philosophy

Engineering

The University of New Mexico

Albuquerque, New Mexico

December, 2011 
(C) 2011, Alan John Nelson 


\section{DEDICATION}

To Kathleen, the love of my life.

And to my family. To my mother Joyce and father Henry, to Uncle Lou and lyay (Aunt Alice), and to my brothers Eric, Jay and Tim, to my sister Rosie and her husband Bill, to my nephew Robby (son of Hip) and his mother Ann. To my nephews on the west coast, Zack, his wife Cyndy and their son Sam, and Ike (sons of Eric), their stepsister Molly and her mother Caren. And especially to my identical twin brother Lou.

I would like to thank them all for their support and encouragement.

And finally, to all MCNP users everywhere who have ever tried to read - and understand - an MCNP manual. Believe me, I feel your pain.

Umpïyeo!! 


\section{ACKNOWLEDGEMENTS}

I would like to acknowledge Dr. Gary Cooper, my faculty advisor, and Dr. Carlos Ruiz of Sandia National Laboratories (PMTS), for their never-ending patience and unwavering support and encouragement during the course of this work. I would also like to thank Carlos for all our countless conversations where he "set me straight" on a myriad of topics. I would also like to thank Dr. Ray Leeper for his steadfast support. In addition, I would like to acknowledge Sandia National Laboratories, and in particular, Dr. Keith Matzen and Dr. John Porter for providing funding for this work. Also, special thanks go to Dr. Gordon Chandler, Dr. Kelly Hahn, and technologists José Torres and Ruth Smelser for providing support and encouragement. Great thanks goes out to Dr. Dave Fehl for all his assistance as well.

And finally, I would like to thank Brent Davis, Irene Garza, Larry Robbins, Dr. Chris Hagen, Dr. Lee Ziegler, Steve Molnar and Tim Meehan of National Security Technologies (NSTec) for not only building the nTOF detectors, but also testing and characterizing them, and providing the support and facilities necessary for calibrating them as well. 


\title{
A Novel Method for Modeling the Neutron Time of Flight (nTOF) \\ Detector Response in Current Mode to Inertial Confinement Fusion Experiments
}

By

\author{
Alan John Nelson \\ B.S., Nuclear Engineering, University of New Mexico, 1988 \\ M.S., Nuclear Engineering, University of New Mexico, 2003 \\ Ph.D., Engineering, University of New Mexico, 2011
}

\begin{abstract}
There are several machines in this country that produce short bursts of neutrons for various applications. A few examples are the Z-machine, operated by Sandia National Laboratories in Albuquerque, $\mathrm{NM}^{\dagger}$; the OMEGA Laser Facility at the University of Rochester in Rochester, NY ${ }^{\S}$; and the National Ignition Facility (NIF) operated by the Department of Energy at Lawrence Livermore National Laboratory in Livermore, California ${ }^{\ddagger}$. They all incorporate neutron time of flight (nTOF) detectors which measure neutron yield, and the shapes of the waveforms from these detectors contain germane information about the plasma conditions that produce the neutrons. However, the signals can also be "clouded" by a certain fraction of neutrons that scatter off structural components and also arrive at the detectors, thereby making analysis of the plasma

\footnotetext{
†'Matzen, K., Phys. Plasmas 4, 1519 (1997).

${ }^{\S}$ T.R. Boehly, D.L. Brown, R.S. Craxton, R.L. Keck, J.P. Knauer, J.H. Kelly, T.J. Kessler, S.A. Kumpan, S.J. Loucks, S.A. Letzring, F.J. Marshall, R.L. McCrory, S.F.B. Mose, W. Seka, J.M. Soures, and C.P. Verdon, "Initial performance results of the OMEGA laser system," Opt. Commun., vol. 133, pp. 495-506, 1997.

"E.I. Moses, "The National Ignition Facility: Status and plans for the experimental program," Fusion Sci. Technol., vol 44, pp. 11-18, 2003.
} 
conditions more difficult. These detectors operate in current mode - i.e., they have no discrimination, and all the photomultiplier anode charges are integrated rather than counted individually as they are in single event counting. Up to now, there has not been a method for modeling an nTOF detector operating in current mode. MCNP-PoliMi ${ }^{\nabla}$ was developed in 2002 to simulate neutron and gamma-ray detection in a plastic scintillator, which produces a collision data output table about each neutron and photon interaction occurring within the scintillator; however, the post-processing code which accompanies MCNP-PoliMi assumes a detector operating in single-event counting mode and not current mode. Therefore, the idea for this work had been born: could a new post-processing code be written to simulate an nTOF detector operating in current mode? And if so, could this process be used to address such issues as the impact of neutron scattering on the primary signal? Also, could it possibly even identify sources of scattering (i.e., structural materials) that could be removed or modified to produce "cleaner" neutron signals?

This process was first developed and then applied to the axial neutron time of flight detectors at the Z-Facility mentioned above. First, MCNP-PoliMi was used to model relevant portions of the facility between the source and the detector locations. To obtain useful statistics, variance reduction was utilized. Then, the resulting collision output table produced by MCNP-PoliMi was further analyzed by a MATLAB postprocessing code. This converted the energy deposited by neutron and photon interactions in the plastic scintillator (i.e., nTOF detector) into light output, in units of

\footnotetext{
${ }^{\nabla}$ S.A. Pozzi, E. Padovani, M. Marsequerra, Nucl. Instr. and Meth. A 513 (2003) 550-558.
} 
$\mathrm{MeVee}^{\phi}$ (electron equivalent) vs time. The time response of the detector was then folded into the signal via another MATLAB code. The simulated response was then compared with experimental data and shown to be in good agreement.

To address the issue of neutron scattering, an "Ideal Case," (i.e., a plastic scintillator was placed at the same distance from the source for each detector location) with no structural components in the problem. This was done to produce as "pure" a neutron signal as possible. The simulated waveform from this "Ideal Case" was then compared with the simulated data from the "Full Scale" geometry (i.e., the detector at the same location, but with all the structural materials now included). The "Ideal Case" was subtracted from the "Full Scale" geometry case, and this was determined to be the contribution due to scattering. The time response was deconvolved out of the empirical data, and the contribution due to scattering was then subtracted out of it. A transformation was then made from $d N / d t$ to $d N / d E$ to obtain neutron spectra at two different detector locations.

$\overline{\phi_{1} \mathrm{MeVee}=\text { amount of light produced }}$ by $1 \mathrm{MeV}$ deposited by a Compton scattered electron. 


\section{TABLE OF CONTENTS}

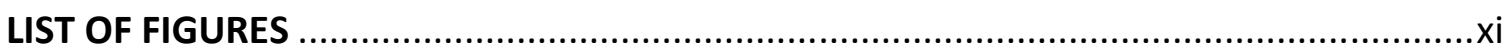

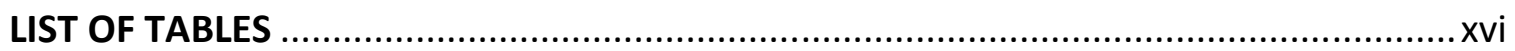

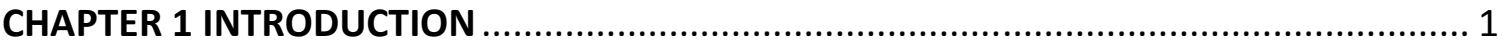

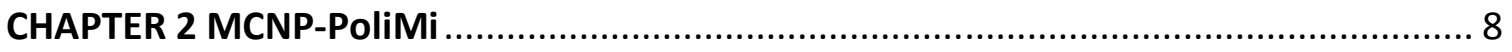

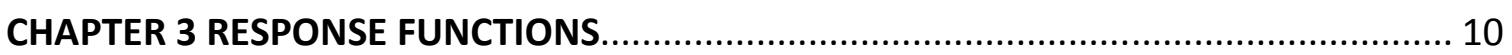

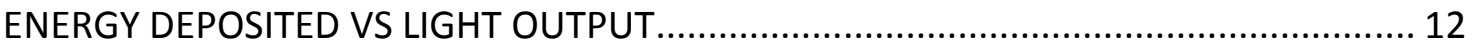

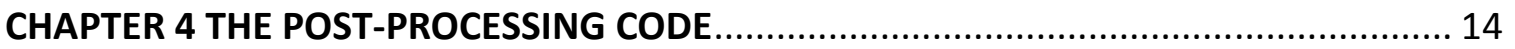

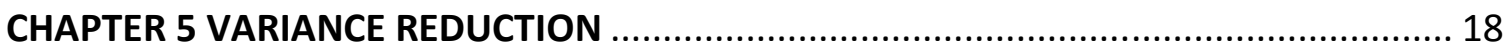

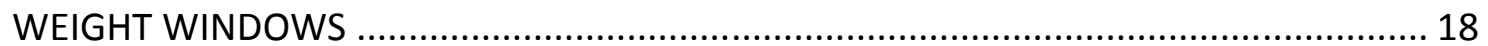

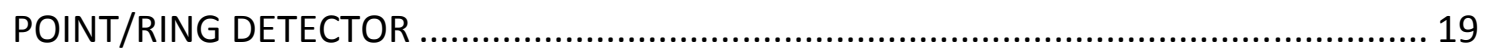

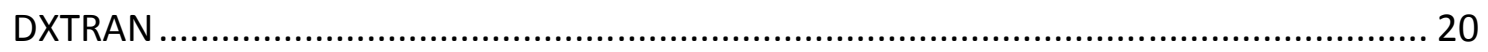

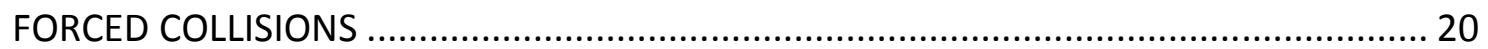

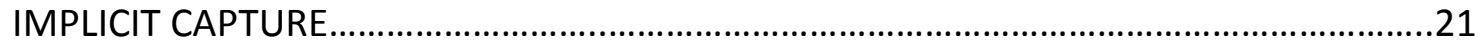

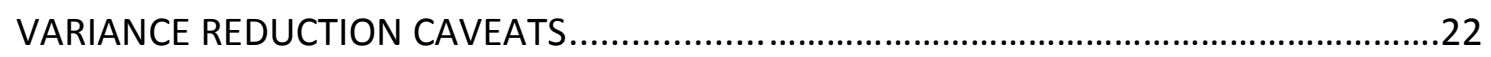

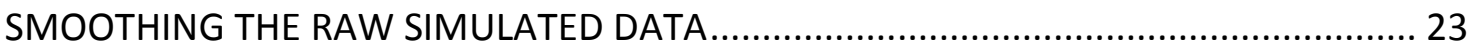

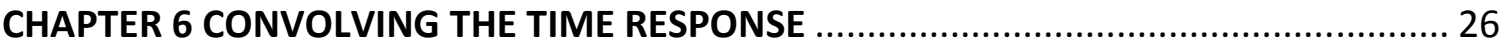

BROADENING DUE TO TEMPERATURE AND TIME RESPONSE................................... 27

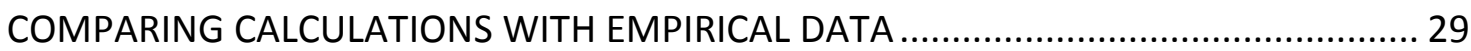

CHAPTER 7 DECONVOLVING THE TIME RESPONSE FROM THE SIMULATED DATA........ 41

CHAPTER 8 CONVOLVING A NEUTRON IMPULSE RESPONSE WITH THE KNOWN TIME

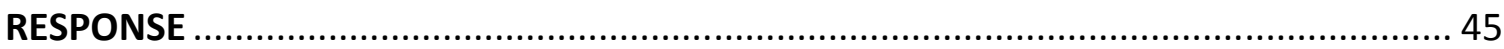


CHAPTER 9 DECONVOLVING THE NEUTRON AND TIMING INSTRUMENT RESPONSE OUT

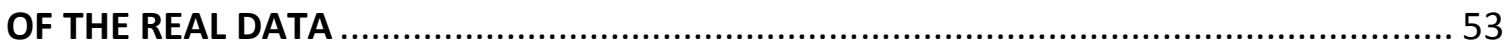

SUBTRACTING THE CONTRIBUTION DUE TO NEUTRON SCATTERING ......................... 53

CHAPTER 10 MAKING THE TRANSFORMATION FROM $(d N / d t)$ to $(d N / d E)$................. 58

CHAPTER 11 IDENTIFYING SOURCES OF NEUTRON SCATTERING ............................. 71

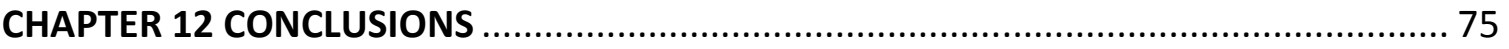

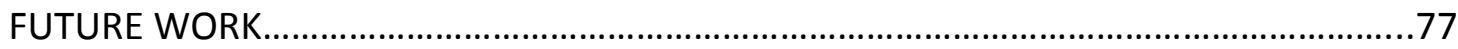

APPENDICIES.

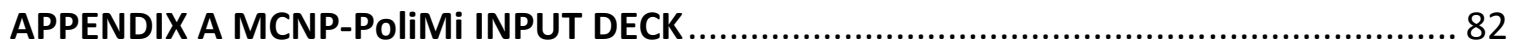

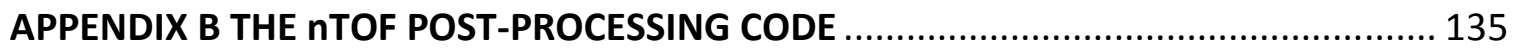

APPENDIX C THE CONVOLUTION ("FOLDING IN") CODE ....................................... 141

APPENDIX D THE DECONVOLUTION ("UNFOLDING") CODE................................. 150

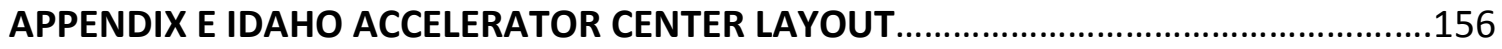

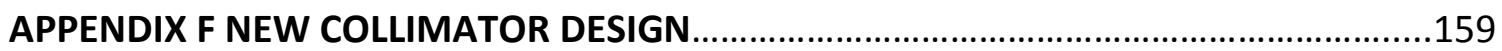

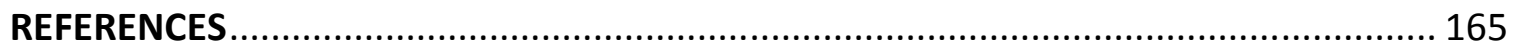




\section{LIST OF FIGURES}

Figure 1. Schematic of nTOF Detector Positions relative to ICF Capsule ........................ 2

Figure 2. Axial Cross Sectional Diagram of the Z-Facility ............................................ 3

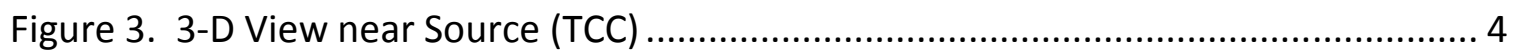

Figure 4. Original Basement "Pig" and its MCNP-PoliMi model ................................... 5

Figure 5. 3-D View of Polyethylene Collimator and Top nTOF ................................. 6

Figure 6. 3-D View of Top and Bottom nTOF Detectors .......................................... 7

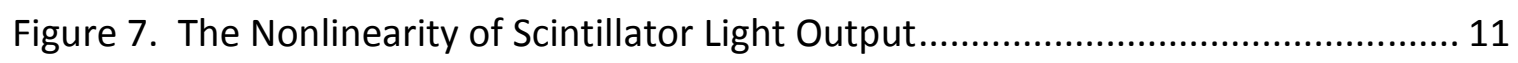

Figure 8. Energy Deposition (MeV) vs Light Output (MeVee) ..................................... 13

Figure 9. Flowchart of the Post-Processing Code .................................................. 15

Figure 10. An analog MCNP-PoliMi model (i.e., without any Variance Reduction

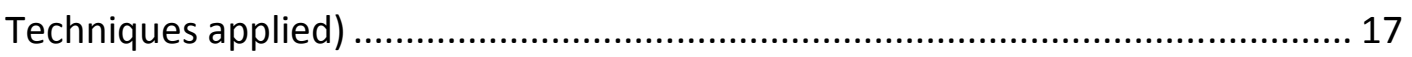

Figure 11. Output of the Post-Processing code for the largest amount of scattering seen in an nTOF signal for this type of experiment ............................................... 24

Figure 12. Smoothing the data with the Savitzky-Golay smoothing filter........................25

Figure 13. Detector time response of an nTOF detector found at the Idaho Accelerator

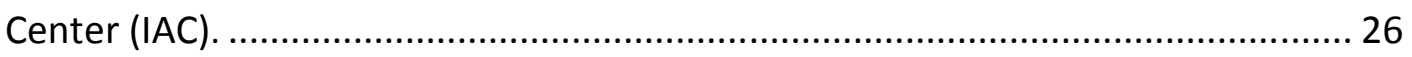

Figure 14. Broadening due to Temperature and Time Response............................... 28

Figure 15. Area normalized comparison between shot z1217 without TIVAR 1000

Collimator (red) and MCNP-PoliMi model with folded-in time response for a $4 \mathrm{keV}$

DD fusion source for a detector located at "D" in Figure 1................................ 30 
Figure 16. Close-up of the primary neutron peak in Figure 15, for the bottom nTOF detector located at "D" in Figure 1.

Figure 17. Area normalized comparison between shot z1217 without TIVAR Collimator (red) and MCNP-PoliMi model with folded-in time response for a 4 keV DD fusion source (blue) for a detector located at " $\mathrm{C}$ " in Figure 1 32

Figure 18. Close-up of the primary neutron peak in Figure 17, for the top nTOF detector located at "C" in Figure 1. .33

Figure 19. A closer view of the UHMW TIVAR 1000 collimator incorporated into the machine on neutron producing shots in order to help "clean up" neutron signals

Figure 20. "Shadow" of TIVAR 1000 Collimator. 35

Figure 21. With the pig aligned 3 degrees off-axis, the collimator "cone" just encompassed both detectors. 36

Figure 22. Area normalized comparison of shot z1549 (red) for detector at location "D" in Figure 1 with TIVAR 1000 Collimator in place and MCNP-PoliMi model with folded in time response of $7.5 \mathrm{~ns}$

Figure 23. Close-up of the primary neutron peak in Figure 22, for the bottom nTOF detector located at "D" in Figure 1, with the TIVAR 1000 Collimator in place 38

Figure 24. Area normalized comparison of shot z1549 (red) for detector at location "C" in Figure 1 with TIVAR 1000 Collimator in place and MCNP-PoliMi model with folded in time response of 7.5 ns.... 
Figure 25. Close-up of the primary neutron peak in Figure 21, for the top nTOF detector located at "C" in Figure 1 with the TIVAR 1000 Collimator in place 40

Figure 26. Preparation for the signal prior to taking Fast Fourier Transforms. 42

Figure 27. "Wrap-Around Order." 43

Figure 28. The smoothed calculated data (blue, also shown in Figure 12 with a red trace) is compared to the deconvolved fit using Fast Fourier Transforms (red). 44

Figure 29. The neutron impulse response for a $2.54 \mathrm{~cm}$ (1 inch) scintillator placed 809 $\mathrm{cm}$ from a monoenergetic source of $2.45 \mathrm{MeV}$ DD neutrons 45

Figure 30. The neutron impulse response divided up into its component parts: primary and secondary scattering 48

Figure 31. The convolution of the neutron impulse response (Figure 29) with the time response (Figure 13) found at the Idaho Accelerator Center (IAC) 50

Figure 32. Schematic of the time delays that need to be taken into account from the time a neutron impinges upon the scintillator to the time an output pulse is generated by the photomultiplier tube and base. 51

Figure 33. The Neutron Impulse/Time Response corrected for the time when radiation first impinges upon the detector 52

Figure 34. The empirical data from z1217 with the neutron impulse and timing information (Figure 31) deconvolved out of it 54

Figure 35. Area Normalized plot of the empirical data from z1217 (blue) compared with Figure 33 and Figure 34 being convolved together (red) as a "check" of the deconvolution 55 
Figure 36. The "Ideal Case" (red) - i.e., a $2.54 \mathrm{~cm}$ (1 inch) scintillator placed $809 \mathrm{~cm}$ away from a 4 keV DD Fusion Source is subtracted out from the "Full Scale" Geometry 56

Figure 37. The empirical data from z1217 with the time response and neutron impulse response deconvolved out of it, and now with the contribution due to scattering (green in Figure 36) subtracted out of it

Figure 38. The Transformation from $d N / d t$ (Figure 34) to $d N / d E$, the neutron spectrum for shot z1217 at detector location "D" in Figure 1

Figure 39. The "Ideal Case" (red) -- i.e., a $2.54 \mathrm{~cm}$ (1 inch) scintillator placed $730 \mathrm{~cm}$ (location "C" in Figure 1) away from a 4 keV DD Fusion Source is subtracted out from the "Full Scale" geometry (blue) to produce the contribution to scattering (green)

Figure 40. Energy Spectra for both the Top and Bottom nTOF detectors at locations " $\mathrm{C}$ " and " $D$ " in Figure 1 from shot z1217. 65

Figure 41. The "Ideal Case" (red) -- i.e., a $2.54 \mathrm{~cm}$ (1 inch) scintillator placed $809 \mathrm{~cm}$ away from a 2 keV DD Fusion Source is subtracted out from the "Full Scale" Geometry (including the TIVAR Collimator). .66

Figure 42. The "Ideal Case" (red) -- i.e., a $2.54 \mathrm{~cm}$ (1 inch) scintillator placed $730 \mathrm{~cm}$ away from a 2 keV DD Fusion Source is subtracted out from the "Full Scale" Geometry (including the TIVAR Collimator). .68

Figure 43. Energy Spectra for both the Top and Bottom nTOF detectors at locations " $\mathrm{C}$ " and "D" in Figure 1 from shot z1549. .70 
Figure 44. Neutrons Scattering after the Primary Pulse, coming in later in time, scattering through the sides of the pig, and scattering up from the floor......

Figure 45. The Elevator made into a "kill zone," eliminating the second "hump" .73

Figure 46. Both the Elevator and sides of the pig made into "kill zones," eliminating both "humps" .74

Figure 47. Idaho Accelerator Center (IAC) Layout.......................................................156

Figure 48. Experimental Time Response found at Idaho Accelerator Center using 50 ps bursts of $x$-rays.

Figure 49. The model of the first collimator used on the Z-machine.............................159

Figure 50. The new collimator design after the Z-machine was refurbished..................160

Figure 51. The model with the old collimator for the bottom nTOF ("D" in Figure 1)...161

Figure 52. The model with the new collimator for the bottom nTOF ("D" in Figure 1).162

Figure 53. The model with the old collimator for the top nTOF ("C" in Figure 1).........163

Figure 54. The model with the new collimator for the top nTOF ("C" in Figure 1)........164 


\section{LIST OF TABLES}

Table I. Excerpt from MCNP-PoliMi Collision Data Output Table ....................................9

Table II. Temporal Broadening due to Scintillator Thickness and Time Response for a $4 \mathrm{keV}$ DD Fusion Source placed at $809 \mathrm{~cm}$

Table III. Broadening due to Scattering at Detector Location " $\mathrm{D}$ " with no Collimator.....57

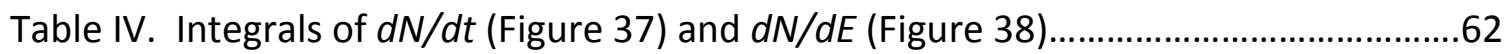

Table V. Broadening due to Scattering at Detector Location " $\mathrm{C}$ " with no Collimator......64

Table VI. Broadening due to Scattering at Detector Location "D" with a Collimator.......67

Table VII. Broadening due to Scattering at Detector Location " $\mathrm{C}$ " with a Collimator.......69

Table VIII. Broadening Due to Scattering for each Detector Location...............................69 


\section{CHAPTER 1}

\section{INTRODUCTION}

Neutron Time of Flight (nTOF) detectors are fielded on neutron producing experiments on Sandia National Laboratories' Z machine [1,2]. Some of these are Inertial Confinement Fusion [3] (ICF) experiments using deuterium filled capsules. In addition, these detectors are used to measure the neutron yield and neutron energy from the reaction:

$$
D+D \rightarrow H e^{3}(0.82 \mathrm{MeV})+n(2.45 \mathrm{MeV})
$$

The detectors consist of $2.54 \mathrm{~cm}$ ( 1 inch) thick by $7.62 \mathrm{~cm}$ ( 3 inch) diameter Bicron 418 plastic scintillator coupled via UVT plastic light guides to fast Hamamatsu R5945 mesh-type photomultiplier tubes. Two of these ("side-on") detectors were located along a single line-of-sight at $102^{\circ}$ with respect to the z-pinch axis at distances of $742 \mathrm{~cm} \mathrm{(24.34} \mathrm{ft)} \mathrm{and} 839 \mathrm{~cm}(27.53 \mathrm{ft})$. Another pair of "on-axis" detectors were located on a single line of sight along the z-axis at distances of $730 \mathrm{~cm}(23.95 \mathrm{ft})$ and 809 $\mathrm{cm}(26.54 \mathrm{ft})$, below the target chamber center (TCC). A schematic of all the nTOF detector positions relative to the ICF capsule is shown in Figure 1.

The physical dimensions of the Z-Facility are quite large, with meters of distance between the source and the detectors. An axial cross-sectional diagram of the facility is shown below in Figure 2, which includes the "on-axis" nTOF detectors located in the basement "pig," which actually had to be fielded $3^{\circ}$ off axis, to allow space for other 


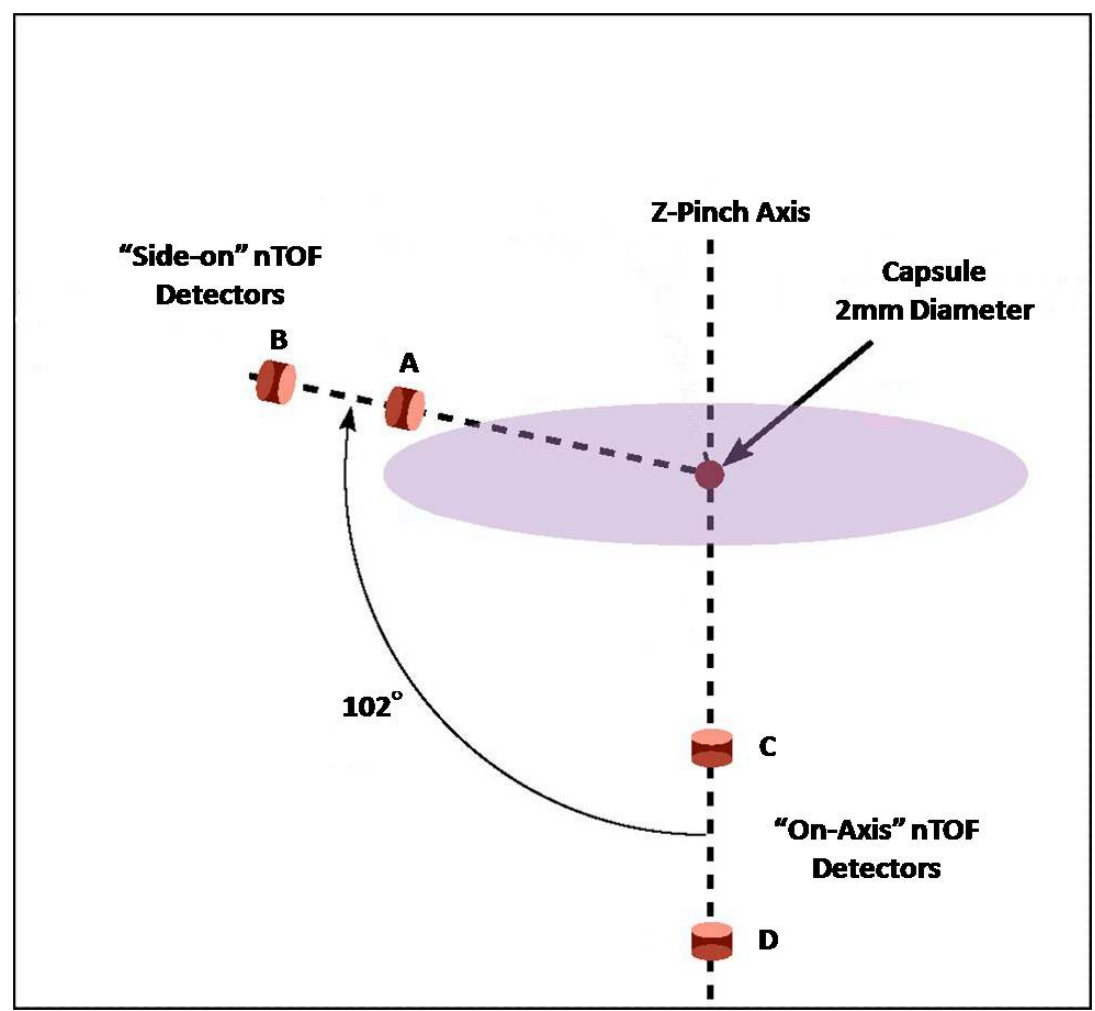

Figure 1. Schematic of nTOF Detector Positions relative to ICF Capsule.

diagnostics sharing the axial view. Due to the intense bremsstrahlung background characteristically produced by Z pinches [4], $20.32 \mathrm{~cm}$ (8 inches) of lead shielding was required to prevent the detectors from producing a non-linear response due to saturation of the PMT in the extreme x-ray pulse and not recovering before the neutron signal arrived.

To acquire realistic nTOF signals at the detector, part of the Z-Facility, particularly between the source (Z-pinch) and detector locations would have to be modeled with MCNP [5], with a reasonable degree of detail. To include the axial nTOF detectors in the basement below TCC, the model would extend from the pinch location downward, comprising three of the magnetically insulated transmission lines (MITLs), the stack (which makes up the vacuum chamber), the bottom lid, and the radiation 


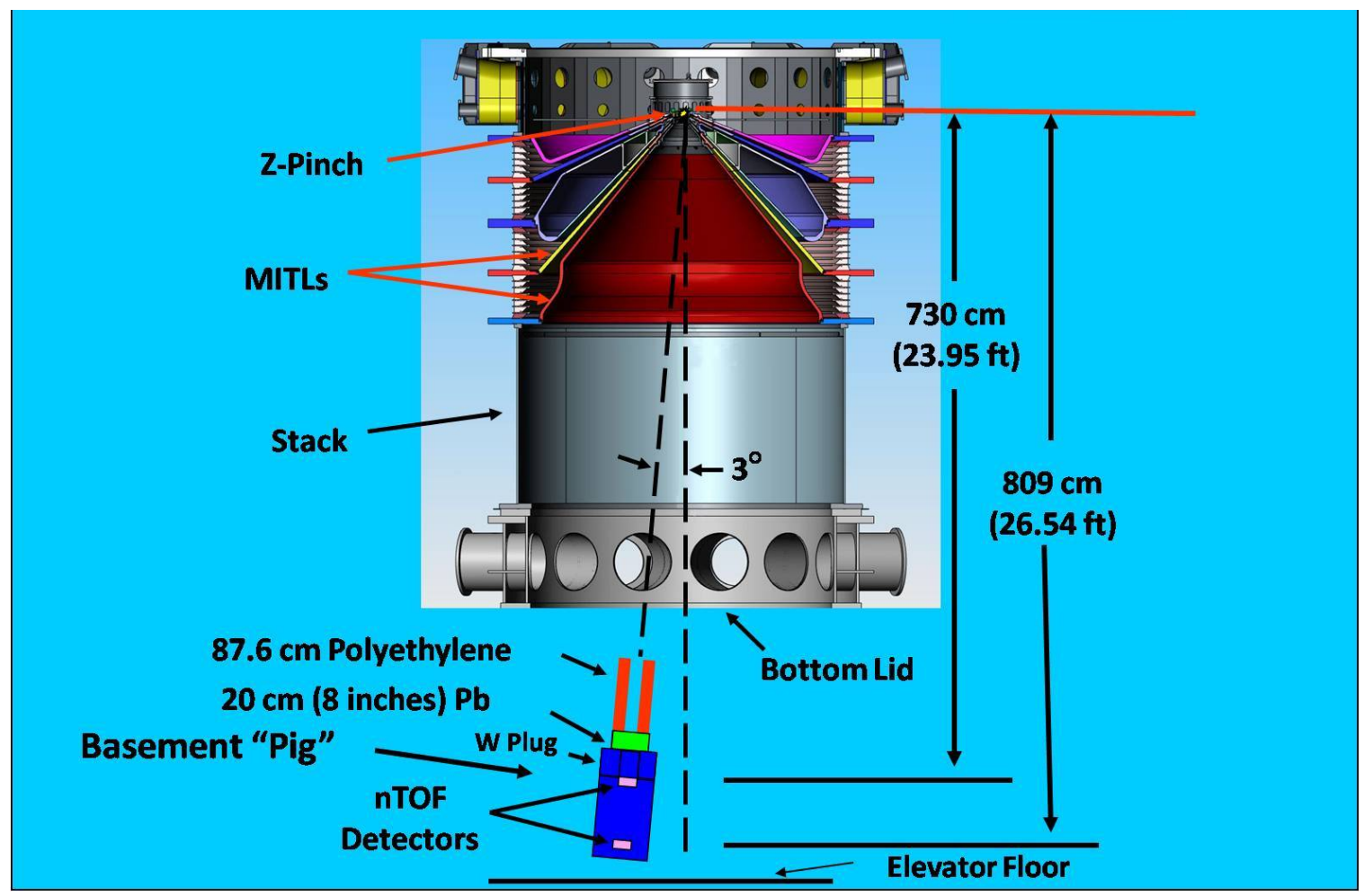

Figure 2. Axial Cross Sectional Diagram of the Z-Facility from the Z-pinch to the Basement "Pig," three degrees off-axis, and $7 \mathrm{~m}-8 \mathrm{~m}(23 \mathrm{ft}-26 \mathrm{ft})$ away from the pinch (TCC).

shield in the basement (i.e., the "pig") housing the two nTOF detectors. In addition to the pig, a polyethylene collimator $87.6 \mathrm{~cm}$ (34.49 in) long with an inner diameter of 7.62 $\mathrm{cm}$ (3in) that was fixed to the top of the pig was also included. A cross section view near TCC of the model used is shown in Figure 3; the entire model comprises over 2400 cells, and more than 900 surfaces. The great number of cells and surfaces were required due to the large scope of the machine. Although the basic geometry of the machine is straightforward - the vacuum chamber is a large cylinder, the MITLs (magnetically insulated transmission lines, shown in Figure 2) are large cones; even the "pig" in the basement has a cylindrical geometry, and these basic shapes all exist within MCNP, however, one cannot assign, for example, one cylindrical cell to be the vacuum 


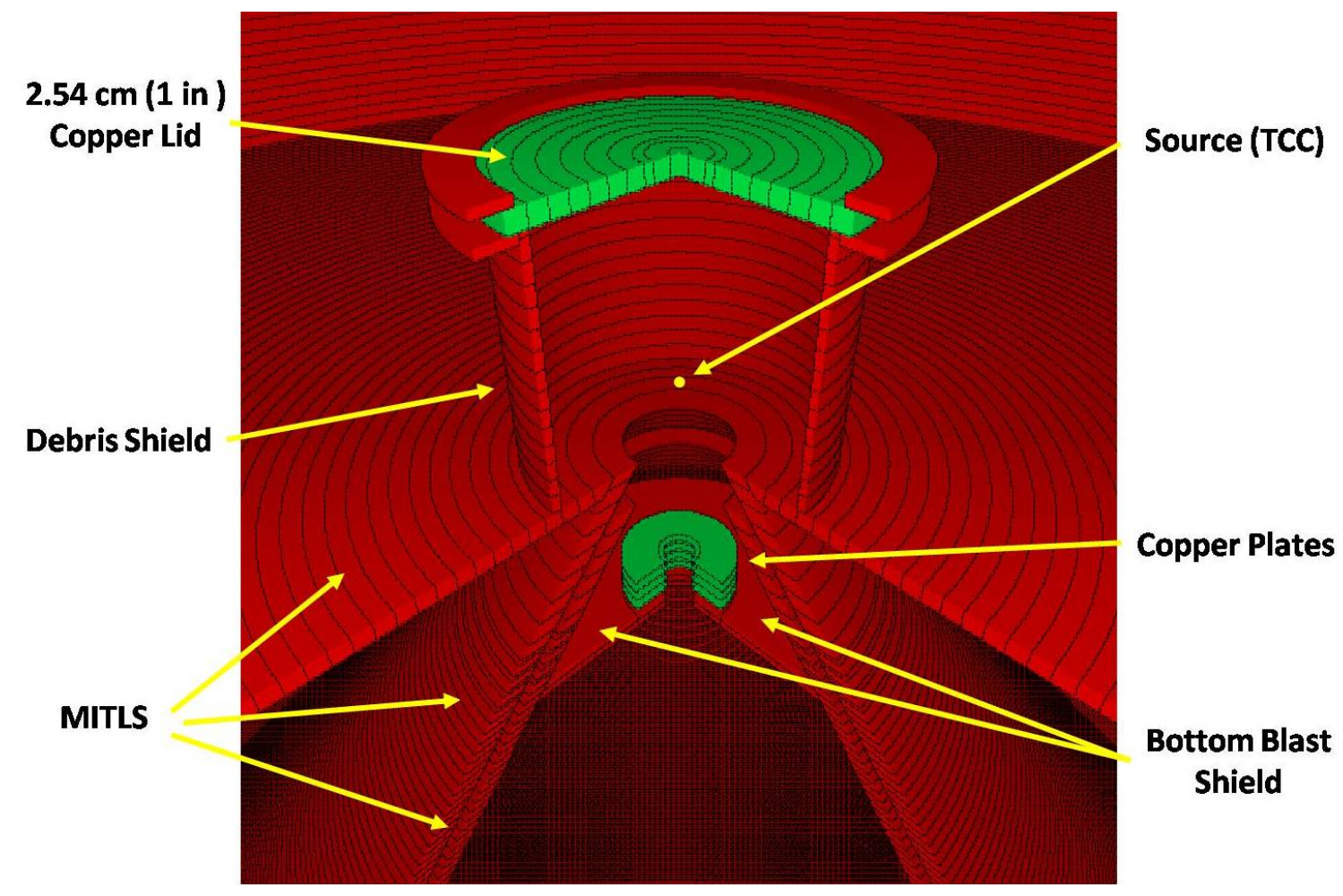

Figure 3. 3-D View near Source (TCC). The MCNP-PoliMi model comprised of over more than 2400 cells and 900 surfaces. The overall geometry is cylindrical, and the lines seen in the figure are individual surfaces making up each slice, or cell.

chamber, with an inner diameter of $3.20 \mathrm{~m}$ (10 ft, 6 inches) and a length from TCC downward of $5.3 \mathrm{~m}$ (17 feet, 5 inches). To track particles effectively, MCNP requires that the optical thickness of cell dimensions be on the order of one mean free path [5]. For DD neutrons of $2.45 \mathrm{MeV}$ through stainless steel, the mean free path is $3.33 \mathrm{~cm}$ (1.31 inches). Therefore, a great number of cells and surfaces were needed to divide the vacuum chamber into thin slices (cells) - the same with the MITLs, and the same with the basement "pig" - in fact, the same with the entire geometry of the problem. Making simple slices of the geometry also allowed MCNP to run faster, since it prefers the problem geometry made up of many simple cells rather than fewer more com- 
plicated cells [5]. The slices can be seen in the three-dimensional view near the source, in Figure 3 . The overall geometry was cylindrical, and the lines seen in Figure 3 are individual surfaces making up each slice, or cell.

The "pig" which housed the two nTOF detectors was originally designed to field an $\mathbf{x}$-ray camera, and was not designed as a neutron shield; however, it was the only shield available at the time, and had ample space to accommodate the two nTOF detectors. Also, being comprised of high Z materials - namely lead and tungsten made it an effective shield against the intense bremsstrahlung background. The original basement pig compared to its MCNP model are shown in Figure 4. As seen in the
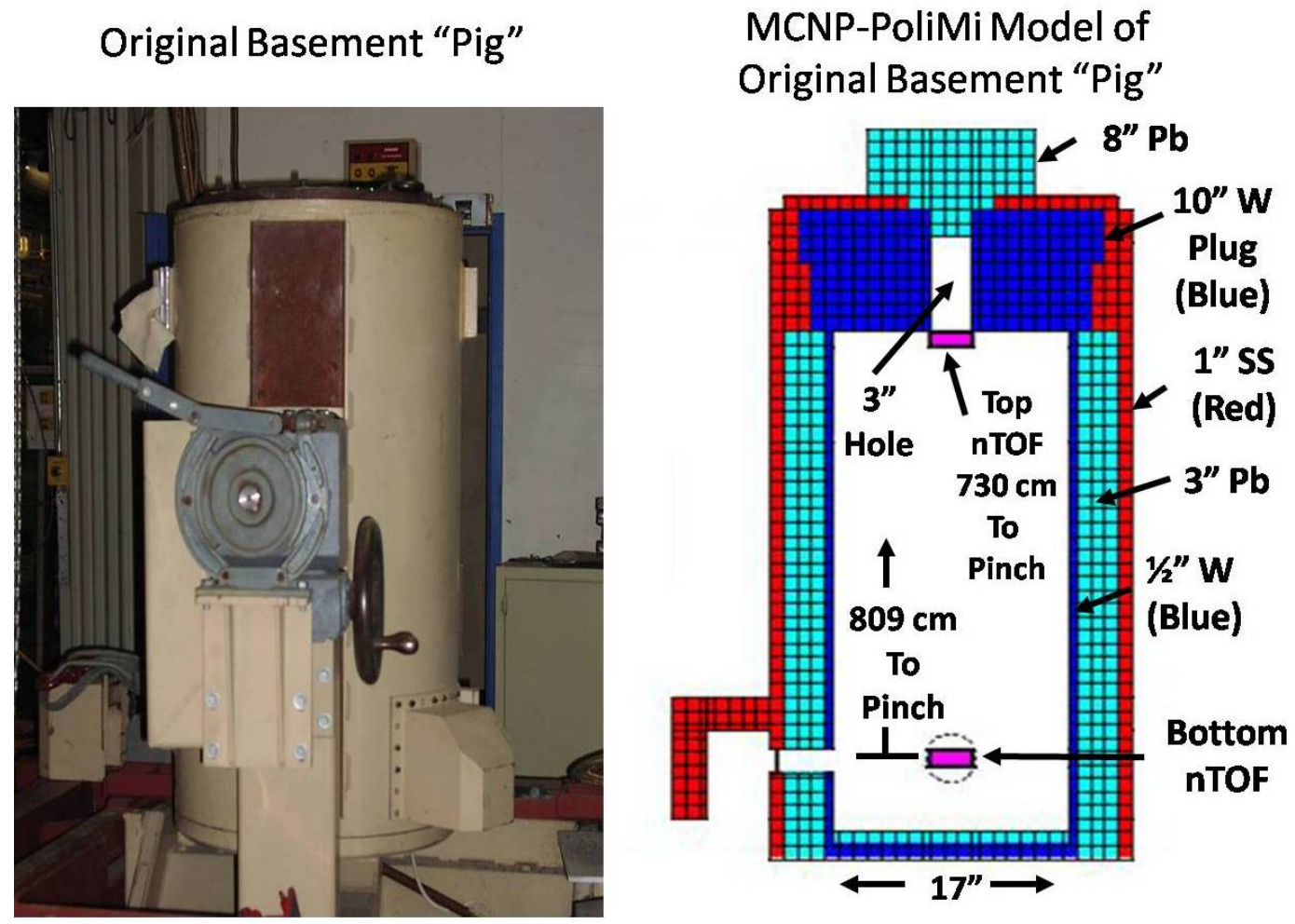

Figure 4. Original Basement "Pig" and its MCNP-PoliMi model. It was not designed as a neutron shield; it originally housed an x-ray camera. The lead plug located on top (right) was necessary to attenuate the intense $x$-ray pulse at shot time so the detectors would not saturate. 
figure, an additional $20.32 \mathrm{~cm}$ ( 8 in) of lead were added to the top of the pig to cover the $7.62 \mathrm{~cm} \mathrm{(3} \mathrm{in)} \mathrm{aperture.} \mathrm{This} \mathrm{shielding} \mathrm{was} \mathrm{necessary} \mathrm{to} \mathrm{reduce} \mathrm{the} \mathrm{bremsstrahlung}$ pulse in the detectors. Without it, that intense $x$-ray pulse would saturate the two nTOFs, and they would not recover in time to see the DD neutron pulse arriving over 300 ns later.

As mentioned above, a polyethylene collimator $87.6 \mathrm{~cm}$ (34.49 in) long with a $7.62 \mathrm{~cm} \mathrm{(3} \mathrm{in)} \mathrm{inner} \mathrm{aperture} \mathrm{was} \mathrm{located} \mathrm{on} \mathrm{top} \mathrm{of} \mathrm{the} \mathrm{pig.} \mathrm{A} \mathrm{cross} \mathrm{section} \mathrm{of} \mathrm{the}$ polyethylene collimator and top of the pig is shown in Figure 5. Below the $20.32 \mathrm{~cm}$ (8

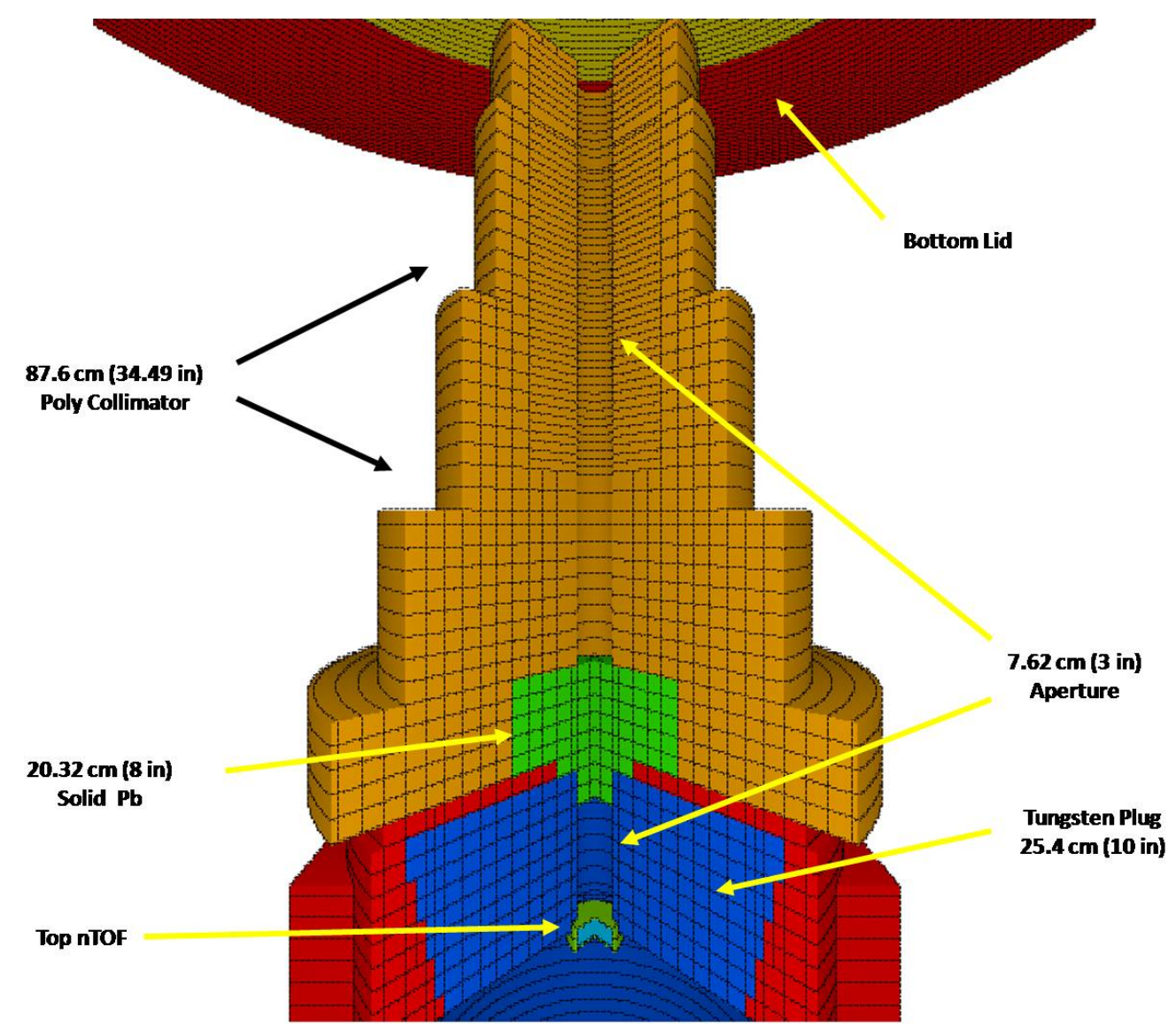

Figure 5. 3-D View of Polyethylene Collimator and Top nTOF. The detector is located at the base of the $7.62 \mathrm{~cm}(3 \mathrm{in})$ aperture. 
in) of lead is a tungsten plug $25.4 \mathrm{~cm}$ (10 in) long with a $7.62 \mathrm{~cm}(3 \mathrm{in})$ aperture. The top nTOF detector is located at the base of the aperture. A cross section of the lower part of the pig showing both the top and bottom nTOF detectors, part of the pig chassis and elevator floor is shown in Figure 6.

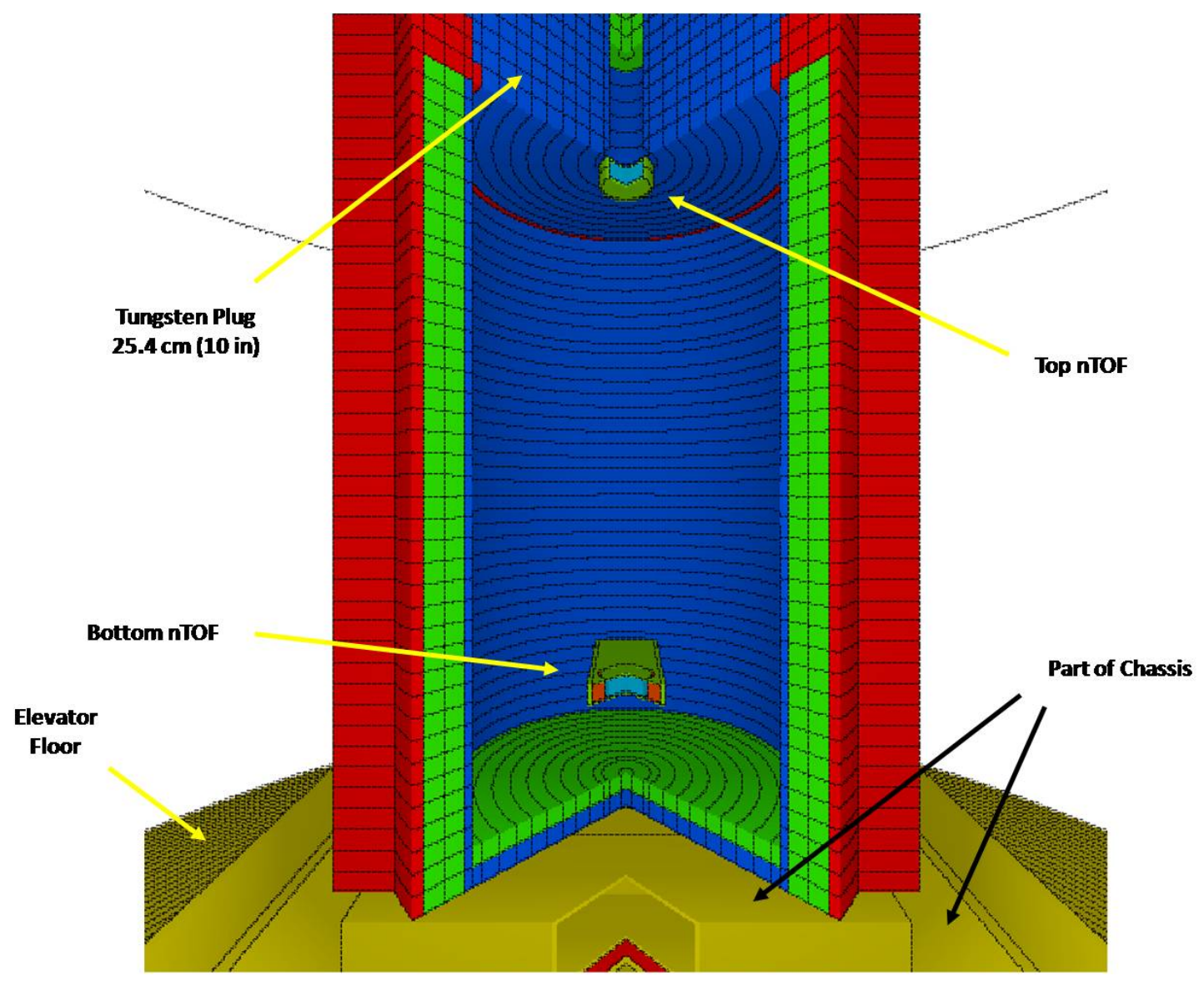

Figure 6. 3-D View of Top and Bottom nTOF Detectors. Part of the chassis, the elevator floor and tungsten plug can also be seen. 


\section{CHAPTER 2}

\section{MCNP-PoliMi}

MCNP-PoliMi [6] is a user-modified version of a general purpose, continuous-

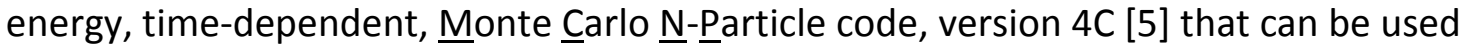
for neutron, photon, electron, or coupled neutron/photon/electron transport. In it, the user creates an input file which contains: the geometry of the problem, description of materials in the problem, the location and characteristics of the source, and the type of answers or tallies desired. It has been used to simulate measurements made by the Nuclear Materials Identification System (NMIS) [7], and has been validated [8]. It was developed at the Polytechnic of Milan, Italy (which gives rise to its name; PoliMi stands for "Politecnico di Milano") by E. Padovani and S.A. Pozzi, in 2002 [9]. It is a versatile tool to simulate particle interactions and detection processes, and consists of two stages: first, an input file is run which produces a collision data output table, then the PoliMi MATLAB post-processing code [9] analyzes the table and produces a detector response. In this case, the MATLAB post-processing code was rewritten for this work to simulate a detector response produced by an nTOF detector operated in current mode $[10]$.

Detailed information about each neutron and photon interaction occurring in user-specified cells is reported in the collision data output table. Interaction type, target nucleus, energy deposited in the collision, time at which the collision occurred, and number of scatterings are among the pertinent data. A partial sample of the collision data output table is shown below in Table I. The modified MATLAB post-processing 
code reads the collision data output table, and converts the energy deposited in MeV to MeVee (electron equivalent) [11] according to the incident particle's response function.

Table I.

Excerpt from MCNP-PoliMi Collision Data Output Table.

\begin{tabular}{|c|c|c|c|c|c|}
\hline$\frac{\text { Projectile }}{\text { Type }^{\S}}$ & $\frac{\text { Interaction }}{\text { Type }^{\nabla}}$ & $\frac{\text { Target }^{+}}{\text {Nucleus }^{\dagger}}$ & $\begin{array}{c}\text { Energy } \\
\text { Deposited in } \\
\text { Collision (MeV) } \\
\end{array}$ & $\frac{\text { Time }}{\text { (Shakes) }^{\Phi}}$ & $\begin{array}{c}\frac{\text { Number }}{\text { Of }} \\
\text { Scatterings } \\
\end{array}$ \\
\hline 1 & -99 & 1001 & 0.52526 & 43.55 & 1 \\
\hline 1 & -99 & 1001 & 0.18983 & 84.74 & 2 \\
\hline 1 & -99 & 1001 & 0.01374 & 84.76 & 1 \\
\hline 1 & -99 & 6000 & 0.01628 & 75.01 & 3 \\
\hline 1 & -99 & 6000 & 0.00892 & 75.13 & 3 \\
\hline 1 & -99 & 1001 & 0.02221 & 75.27 & 3 \\
\hline 1 & -99 & 1001 & 0.01146 & 75.31 & 4 \\
\hline 1 & -99 & 6000 & 0.00028 & 75.43 & 2 \\
\hline 1 & -99 & 1001 & 0.00036 & 75.49 & 1 \\
\hline 1 & -99 & 1001 & 0.00080 & 78.30 & 3 \\
\hline 1 & -99 & 6000 & 0.00012 & 78.74 & 2 \\
\hline 1 & -99 & 6000 & 0.01170 & 74.81 & 1 \\
\hline 2 & 1 & 6 & 1.94631 & 62.74 & 1 \\
\hline
\end{tabular}

${ }^{\S} 1$ = Neutron; 2 = photon; ${ }^{\nabla}-99$ = elastic scattering; 1 = Compton scattering; -1 = inelastic scattering; ${ }^{\dagger} 1001$ and $1=$ Hydrogen; 6000 and $6=$ Carbon; ${ }^{\Phi} 1$ Shake $=10 \mathrm{~ns}=10^{-8} \mathrm{sec}$. 
CHAPTER 3

\section{RESPONSE FUNCTIONS}

The simulation of the detector pulse requires that the energy deposited in the detector by neutrons and photons be converted into light output by using measured detector response functions [9]. Neutrons are detected primarily by elastic scattering on hydrogen, with the measured response function fit to the following quadratic equation for a plastic scintillator as shown in equation (2):

$$
L=0.0364 * E_{n}{ }^{2}+0.125 * E_{n}
$$

where $E_{n}$ is the energy deposited by the neutron on hydrogen (MeV) and $L$ is the measured light output (MeVee). The resulting recoil protons quickly transfer their kinetic energy to luminescent states in the scintillator [12]. Neutron interactions with carbon are assumed to generate a very small light output equal to:

$$
L=0.02 * E_{n}
$$

where $E_{n}$ is the energy deposited by the neutron on carbon (MeV) and $\mathrm{L}$ is the corresponding light output (MeVee). (This is an approximation by the authors of PoliMi, due to the fact that the light conversion of the recoil carbons is roughly one order of magnitude lower than that of the recoil protons; in the post-processing program they arbitrarily imposed that the kinetic energy of carbon nuclei be converted to light with a constant efficiency factor of $0.02 \mathrm{MeVee}$ per MeV [13]). In these reactions, energy is lost by the neutron without significant light production.

Photons, on the other hand, are detected primarily by Compton scattering, and the pulse-height to energy deposited response is very close to linear: 


$$
L=E_{\gamma}
$$

where $E_{\gamma}$ is the energy deposited by the photon (MeV) and $\mathrm{L}$ is the measured light output (MeVee). A plot of the response function for a neutron on hydrogen according to the response functions of PoliMi (equation 2 above) and Stanton [14] as well as Czirr [15] and Verbinski [16] are shown in Figure 7. This displays not only the comparison of PoliMi's, Stanton's, Czirr's and Verbinski's response functions, but also the nonlinear nature of scintillator light output for non-photons.
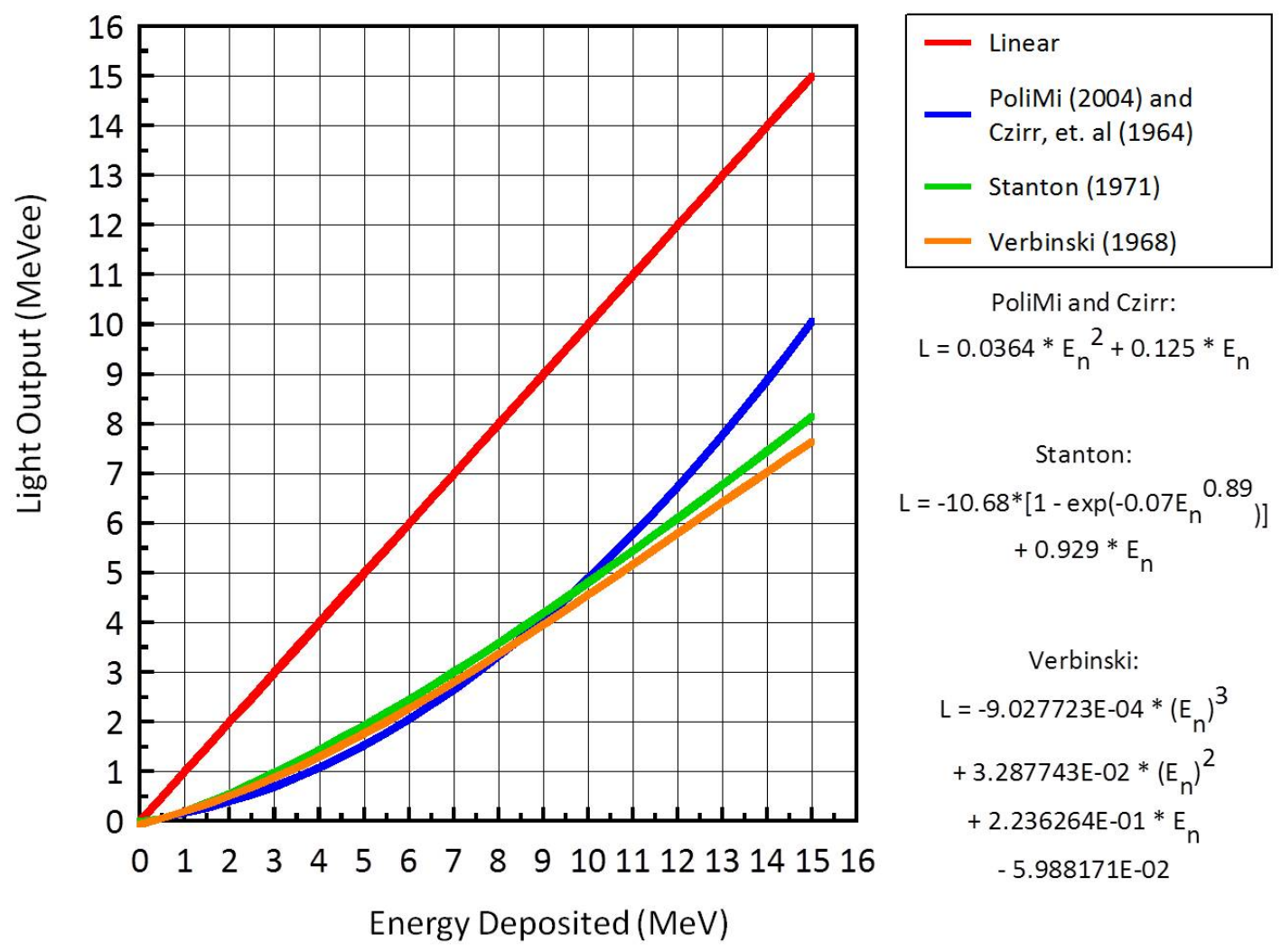

Figure 7. The nonlinearity of Scintillator Light Output. Comparison of the response functions of PoliMi, Czirr, Stanton and Verbinski for a neutron on hydrogen. PoliMi and Czirr compare well with each other, and Stanton and Verbinski are similar. They all produce light output which is decidedly nonlinear in nature. 


\section{ENERGY DEPOSITED VS LIGHT OUTPUT}

There has been a trend in the community when doing calculations of this nature to only look at the energy deposited in the scintillator as opposed to the light output produced from neutron interactions with hydrogen and carbon (in MeVee). Certainly, MCNP accommodates energy deposition tallies, such as an F6 (MeV/gram) [5], but in reality, this is not the true "output" from the scintillator. Without the correct response functions listed above, which have been measured by Stanton [14], Czirr [15], Verbinski [16] and Polimi [17] which convert energy deposited (MeV) to light output (MeVee), one will grossly overestimate the amount of light output which is produced. An illustrative example is shown in Figure 8. For the same MCNPX [18] calculation, the F6 Tally (MeV/gram) was compared to the light output produced by the process discussed in this work. At the left in Figure 8 is the F6 Tally (red) compared to the calculated light output (blue). As can be seen, the F6 Tally crudely resembles the calculated light output, but once they are area normalized (on the right) there is no longer any resemblance, showing that the F6 Tally is overestimating the amount of light output produced greater than an order of magnitude. When the calculated light output in Figure 8 (blue, left) is compared with the actual data and area normalized (as shown, for example, in Figure 15 on page 30) one can see that the calculation is very close to the data, both in terms of shape and magnitude. 

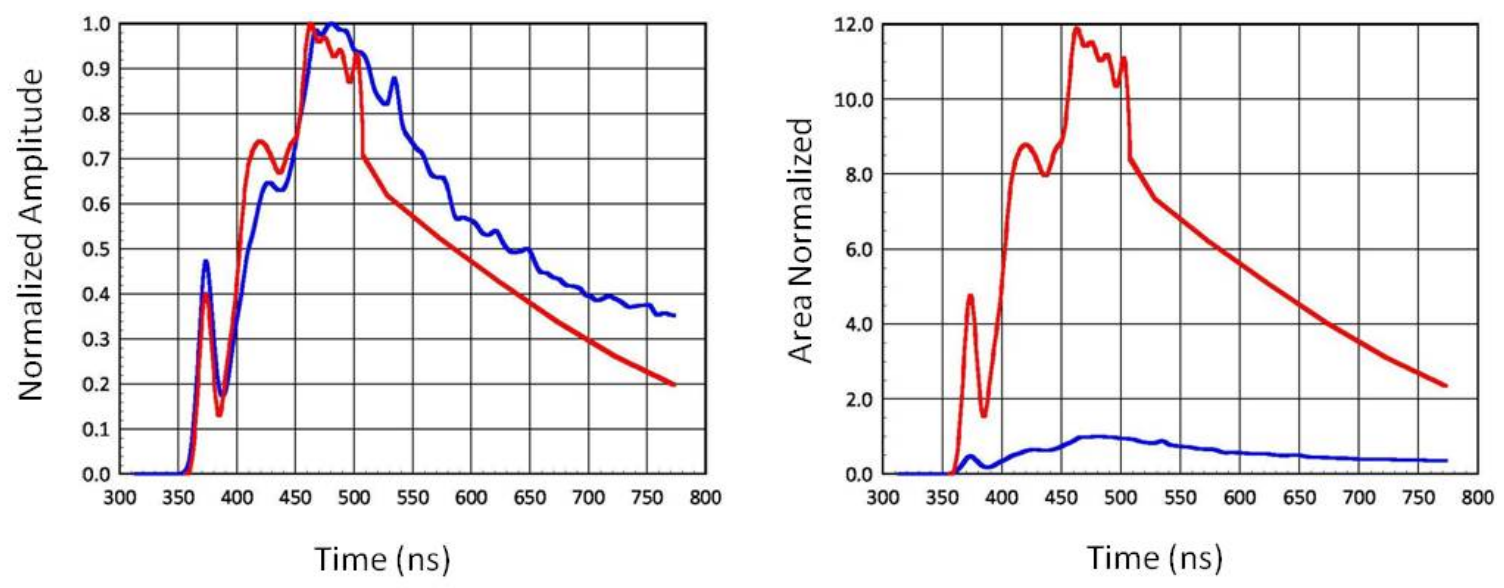

Figure 8. Energy Deposition (MeV) vs Light Output (MeVee). For the same number of histories, light output was produced using the response functions above, and shown in blue; this is compared to an F6 energy deposition tally in MCNPX (MeV/gram, red). On the left one can see that the red crudely resembles the blue in shape, but once they are area normalized (on the right) there is no longer any resemblance, showing that the F6 Tally is overestimating the amount of light produced greater than an order of magnitude. When the calculated light output (blue, left) is compared to the actual data and area normalized (Figure 15, p. 30) the calculation compares well to the data, both in terms of shape and magnitude. 


\section{CHAPTER 4}

\section{THE POST-PROCESSING CODE}

The post-processing code was written in MATLAB, which loads the collision data output table, then sorts it in terms of increasing time (column 6 in Table I, p. 9), then converts the energy deposited in MeV into light output (MeVee) according to the incident particle (either a neutron or photon) and the target nucleus ( $\mathrm{H}$ or $\mathrm{C})$, then sums all the light outputs into time bins which correspond to the resolution of the data digitizer recording (in this case, 200 ps time bins used on Tektronix TVS645 digitizers [19]), then plots the light output versus time. An additional code written for this work convolves the actual time response of the detector with the MATLAB output where it can be compared with empirical data. A flowchart of the post-processing code with these additional steps is shown in Figure 9.

Figure 10 shows an early plot of light output versus time only (with no convolved detector response) for an analog Monte Carlo run. The term "analog" means assigning weight equal to unity to all of the particles generated at the source and to each of the secondary particles born at a collision. The analog model is the simplest Monte Carlo model for particle transport problems because it uses natural probabilities that various events occur (e.g., collision, capture, scattering, etc.). Particles are followed from event to event, and the next event is always sampled (using the random number generator) from a number of possible next events according to the natural event probabilities. 


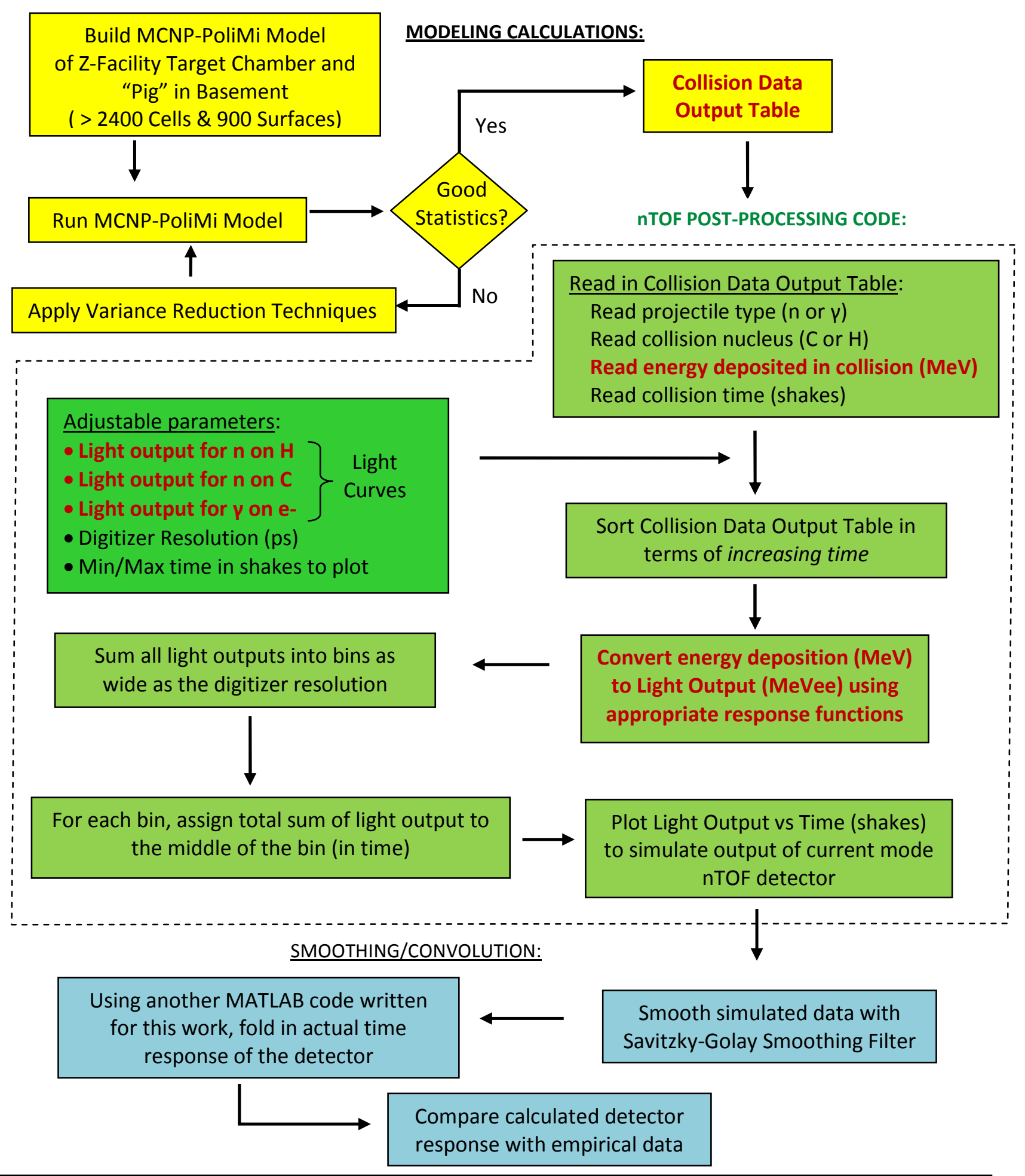

Figure 9. Flowchart of the post-processing code. It reads the collision output table produced in an MCNP-PoliMi model, sorts is in terms of increasing time, converts energy deposited (MeV) into light output (MeVee) with appropriate response functions, sums the light output into time bins equal to the digitizer's resolution, and plots the result. The raw data is then smoothed with a Savitzky-Golay smoothing filter. An additional code then convolves the smoothed data with the actual time response of the detector, where it can then be compared with empirical data. 
This way, analog Monte Carlo is directly analogous to naturally occurring transport. It works well when a significant fraction of the particles contribute to the tally estimate; however, in most real-world type problems with complicated geometries and large source-to-detector distances, the fraction of particles detected can be very small (less than $10^{-06}$ ). For these cases analog Monte Carlo fails because few, if any, of the particles get tallied, and the statistical uncertainty in the answer is unacceptable. The MCNP results in Figure 10 is a case in point; on a desktop PC, it ran the maximum amount of particles (2.0E09), and it took 43 hours. Due to the distance from the source ( $8 \mathrm{~m})$ and amount of material between the detectors and source $(20.32 \mathrm{~cm} \mathrm{~Pb}$, the bottom lid, etc.), the probability of transporting a particle from the Z-pinch (TCC) to an nTOF detector in the basement becomes vanishingly small when using analog Monte Carlo. Therefore, non-analog Monte Carlo techniques had to be implemented. Nonanalog Monte Carlo models estimate the same average value as the analog Monte Carlo model, but often make the variance (uncertainty) of the estimate much smaller than the variance for the analog estimate. In practical terms, this means that problems that would be impossible to solve in days of computer time can now be solved in minutes of computer time.

There are many non-analog techniques, and they all are meant to increase the odds that a particle contributes to a tally. To ensure that the average score is the same in the nonanalog model as in the analog model, the score is modified to remove the effect of biasing (changing) the natural odds. Thus, if a particle is artificially made $q$ times as likely to execute a given random walk (i.e., travel in a particular direction 
toward a detector), then the particle's score is weighted by (multiplied by) $1 / q$. The average score is thus preserved, because it is the sum over all random walks. In this way, nonanalog - or variance reduction - techniques (VRTs) can often decrease the

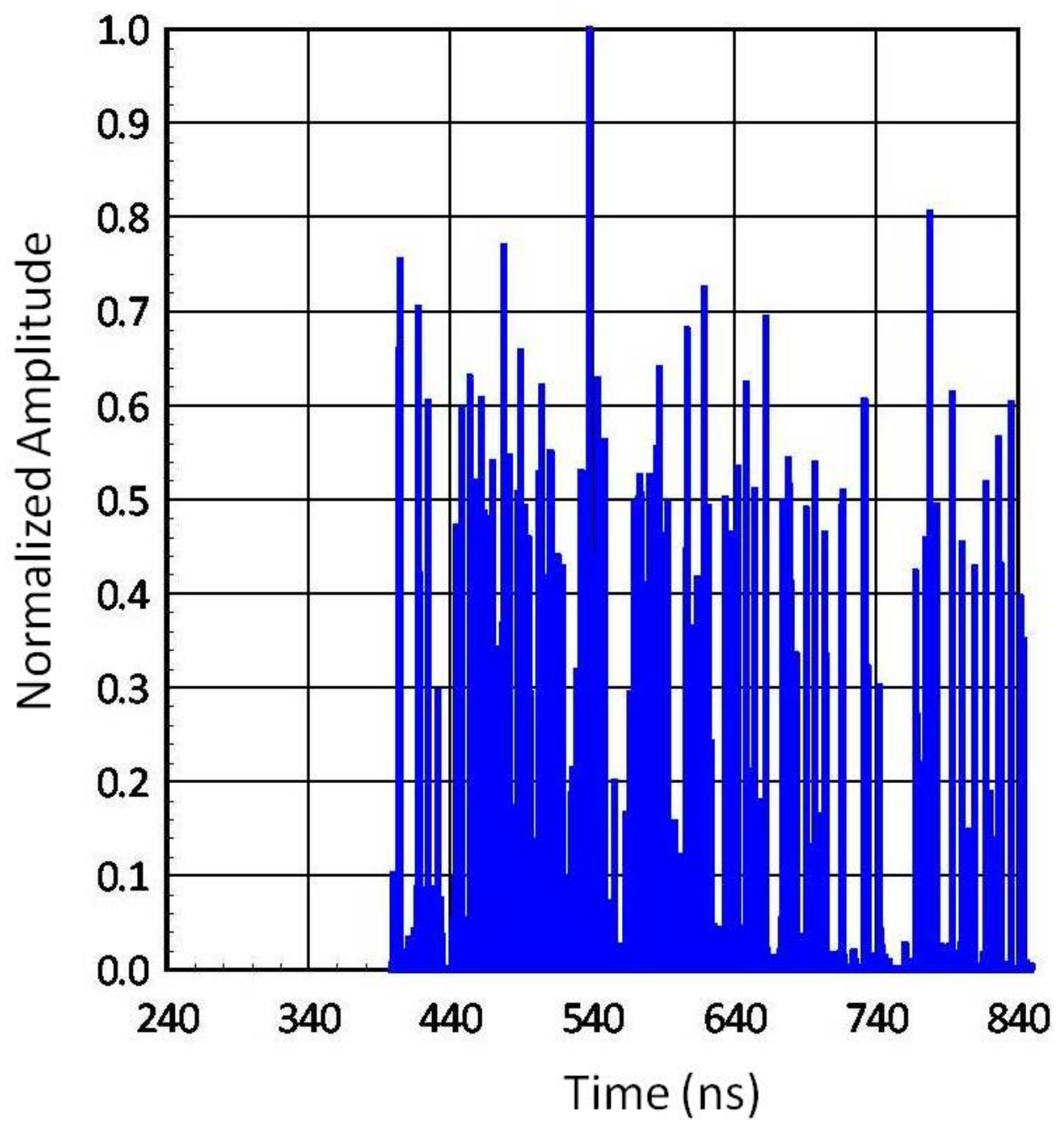

Figure 10. An analog MCNP-PoliMi model (i.e., without any Variance Reduction Techniques applied). This was run with the maximum amount of particles (2.0E09), and it took 43 hours of computer time.

relative error by sampling naturally rare events with an unnaturally high frequency and weighting the tallies appropriately. 


\section{CHAPTER 5}

\section{VARIANCE REDUCTION}

Central to the art of variance reduction is the concept of particle weight [20]. To simulate the transport of a large number of particles, it is not necessary to follow all of them. Rather, it is only necessary to follow a statistically significant sample of particle "histories." Each history is assigned a weight that, in some sense, represents the number of particles modeled. At any time during the random walk of the particle, it may be split into N particle "tracks" provided that the weight is divided by N.

Alternatively, it may be killed with probability 1/N ("Russian Roulette") at any time provided the weight of surviving particles is multiplied by N. All variance reduction schemes work by putting a large number of particles of low weight in regions of interest and allowing only a small number of particles with high weight in unimportant regions of the problem. A summary of all the VRTs that proved useful for this work are shown below.

\section{WEIGHT WINDOWS}

The weight windows method is a population control method which artificially increases/decreases the number of particles in spatial or energy regions that are important/unimportant to the tally score. It is another form of geometry splitting and Russian Roulette, where a particle crossing into a cell of higher importance is split, whereas a particle crossing into a cell of lower importance undergoes Russian Roulette. In this way, particles from the source migrate toward the tally region. The user can also employ a mesh-based weight window (or "importance") generator, where a mesh is 
superimposed over the entire geometry of the problem; this causes an optimum performance function to be generated. This importance function is usually superior to anything an experienced user can guess for cell importances (especially when thousands of cells make up the problem, manually assigning an importance to each one becomes non-trivial). All regions in the problem are assigned a set of upper and lower weight window bounds. Particles with weights greater than the upper bound are split so that all split particles are within the window; particles with weights below the lower bound play Russian Roulette to increase their weight until they lie within the window or are killed [21]. This causes more particles with lower weight to drift toward the tally region.

\section{POINT/RING DETECTOR}

The use of a point detector (or a ring detector if the problem has axial symmetry) is a partially deterministic method where the random walk process is replaced by a deterministic process to move particles from one region to another. It is a necessity in situations where the analog random walk is inefficient. Often, the point is in a region far from the source in an area where it would otherwise be difficult to transport particles. It deterministically estimates the fluence at the specified point in the problem. At every collision site, the probability of a particle scattering toward the point detector is calculated. There are three factors that affect this probability: the distance between the collision site and the point/ring detector; the probability of scattering toward the point/ring detector, rather than in the original direction; and the optical thickness of material between the collision site and the point/ring detector. In this case the point detector was placed at the center of each $7.62 \mathrm{~cm}$ (3 in) diameter, $2.54 \mathrm{~cm}(1 \mathrm{in})$ thick 
plastic nTOF scintillator. However, to eliminate cross-talk, only one point detector was used at a time. Also, since the bottom pig was $3^{\circ}$ off axis as shown in Figure 2, a ring detector could not be used (the problem was not axially symmetric); a point detector was used instead.

\section{DXTRAN}

Like the point/ring detector, DXTRAN is a partially deterministic method. It stands for "deterministic transport," and is a "next event estimator" which is used to deterministically transport the uncollided weight from collision and source points to a spherical surface, known as a DXTRAN sphere. Thus, source particles upon being born, or upon collision during their random walk, generate "pseudoparticles" which are deterministically transported, without collision, to the DXTRAN sphere. The random walk is then continued inside the sphere for these DXTRAN particles. If non-DXTRAN particles try to enter the DXTRAN sphere, they are killed (i.e., removed from the problem) to balance the particle weight contribution to the cells inside the sphere. In this way, one can obtain many particles in a small region of interest that would otherwise be difficult to sample. For this case, a DXTRAN sphere was made just to encompass each 7.62 ( 3 in) diameter, $2.54 \mathrm{~cm}(1 \mathrm{in})$ thick plastic nTOF scintillator. And similarly with using point detectors, only one DXTRAN sphere was used at a time to eliminate cross-talk between multiple spheres.

\section{FORCED COLLISIONS}

Forced Collisions is a modified sampling method which artificially increases the sampling of collisions in specified cells, generally those near a DXTRAN sphere and/or 
point/ring detector. This method splits particles into collided and uncollided parts, where the collided part is forced to interact within the specified cell while the uncollided particle exits the cell without collision. In combination with a DXTRAN sphere and a point/ring detector, this method produces large numbers of collisions which are desirable to more efficiently approach the problem solution. For this model, the specified cell on the forced collisions card was the cell assigned to the actual nTOF plastic scintillator with a point detector located at its center, which was encompassed by a DXTRAN sphere.

\section{IMPLICIT CAPTURE}

Like Forced Collisions, implicit capture is a modified sampling method. When a particle collides, there is a probability that it is captured by the nucleus. In analog capture, the particle is killed with that probability. In implicit capture (also known as "survival biasing," and "absorption by weight reduction") the particle is never killed by capture; instead, its weight is reduced by the capture probability at each collision. In this way, no particles are lost to absorption, but absorption effects are properly accounted for. The advantage of implicit capture is that important particles are not killed after a great deal of effort has been expended to transport them long distances, and that when a particle has finally, against considerable odds, reached the tally region, it is not absorbed just before a tally contribution is made. Also, particles that loose energy through multiple collisions and are no longer considered useful, analog capture can efficiently get rid of them - the user can specify the energy at which analog capture 
takes over. In fact, implicit capture is so powerful that it is one of two MCNP variance reduction options that is turned on by default. The other is Russian Roulette [5].

Using the above variance reduction methods, generally two runs were required to satisfy the requirements of a "good" calculation, namely, that the relative error on the point/ring detector tally (located at the center of the nTOF scintillators) were less than $5 \%$, the Figure of Merit (FOM), or measure of efficiency, was maximized, and that all ten statistical checks in the output were passed. The Figure of Merit is defined as:

$$
F O M=1 / R^{2} T
$$

where $T$ is the run time, and $R$ is the relative error generated by the point/ring detector tally. For different VRTs, the one with the largest FOM is preferred.

\section{VARIANCE REDUCTION CAVEATS}

While some problems can only be solved by using variance reduction methods, the user should proceed cautiously when applying them. When they are used correctly they can greatly help the user produce a more efficient calculation. Used poorly, however, and they can result in a wrong answer with good statistics and few clues that anything is amiss. The user should proceed cautiously when applying VRTs. A few precautions a user should heed when using VRTs are the following:

- The user should err on the conservative side when using VRTs (some techniques are not recommended for the inexperienced user, such as forced collisions, point/ring detectors, and DXTRAN spheres).

- The output should be studied for peculiarities (large fluctuations, etc.) 
- One of the key parameters for assessing the effectiveness of a VRT is the Figure of Merit (FOM) - generally the better the improvement of the FOM, the better is the VRT.

- Also, the FOM table should not be erratic; this indicates poor sampling. The FOM should rapidly approach a constant value (except for fluctuations early on in the simulation).

\section{SMOOTHING THE RAW SIMULATED DATA}

Once the variance reduction techniques listed above were implemented, and the output was examined to make sure the relative error on the point detector tally was $<5 \%$, the FOM was maximized, and the ten statistical checks passed, the postprocessing code was used to plot light output (MeVee) vs time (ns). An example of a plot produced is shown in Figure 11 for detector location " $D$ " as indicated in Figure 1. It should be noted that this data indicated the largest amount of scattering seen in an nTOF signal for this type of experiment. Note that after the initial neutron peak there is a very large, second scattering peak. This was a model of the machine as shown in Figure 3. MCNP- PoliMi models were run at each axial detector location (" $C$ " and " $D$ " in Figure 1), each before and after the collimator was implemented, and will be compared with the experimental data in Chapter 6.

As can be seen in Figure 11, despite all the efforts with variance reduction to obtain as good a signal as possible at the detector, due to the complexity of the problem with large source-to-detector distances, and abundant scattering material throughout 


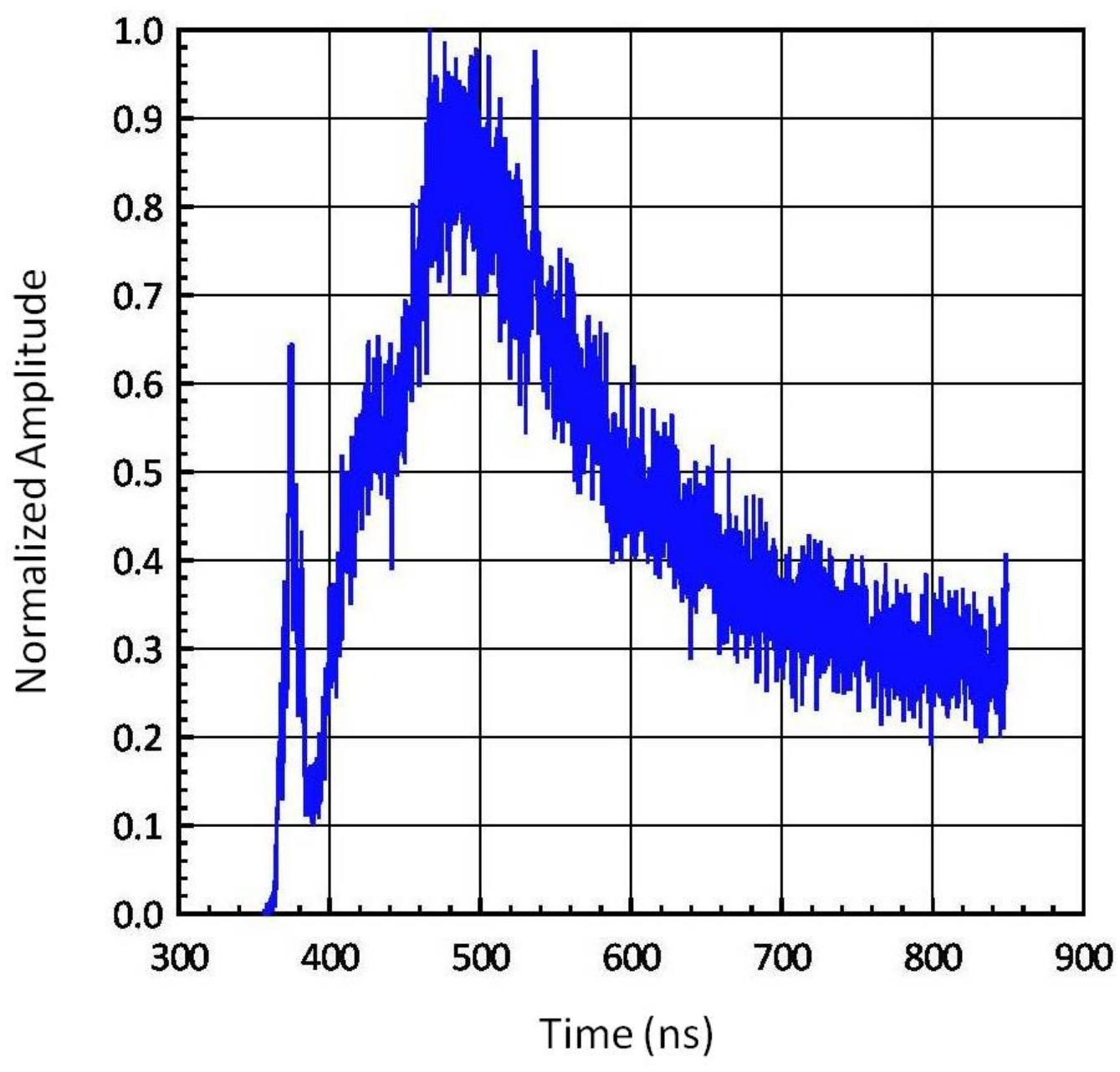

Figure 11. Output of the post-processing code for the largest amount of scattering seen in an nTOF signal for this type of experiment. Light output in MeVee is plotted vs time in ns. This particular case is for detector location " $D$ " in Figure 1. Note that after the primary neutron peak there is a very large, second scattering peak.

the model, the raw simulated data is too noisy. To smooth out this noise, a SavitzkyGolay smoothing filter was used [22]. The advantage of this method is that it tends to preserve features of the distribution such as relative minima, maxima, and width, which are usually 'flattened' by other adjacent averaging techniques [23]. The raw simulated data before and after smoothing are shown in Figure 12. 


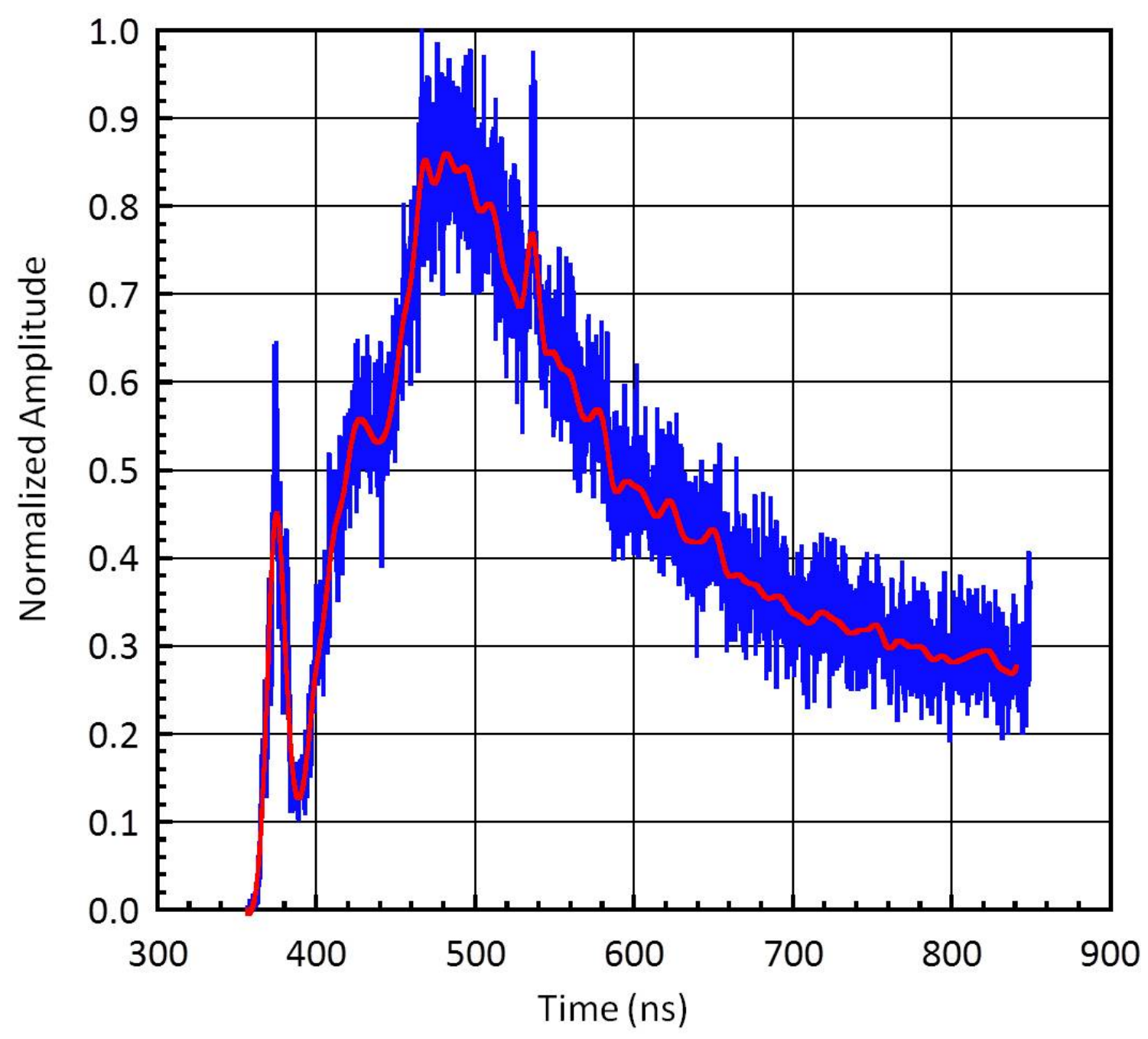

Figure 12. Despite using numerous variance reduction techniques to obtain the best signal possible at the detector, the raw simulated data from Figure 11 (blue) is still too noisy. The Savitzky-Golay smoothing filter was applied to help smooth out the noisy simulated data and is shown in red. 


\section{CHAPTER 6}

\section{CONVOLVING THE TIME RESPONSE}

The next step was to "fold in" - or convolve - the actual time response of the detector with the post-processor output. This required another code to be written in MATLAB. The time response of the detectors used was found experimentally at the Idaho Accelerator Center (IAC) using their $15 \mathrm{MeV}$ Linac producing a 50 ps photon beam [24]; (see also Appendix E). A plot of a detector time response is shown in Figure 13.

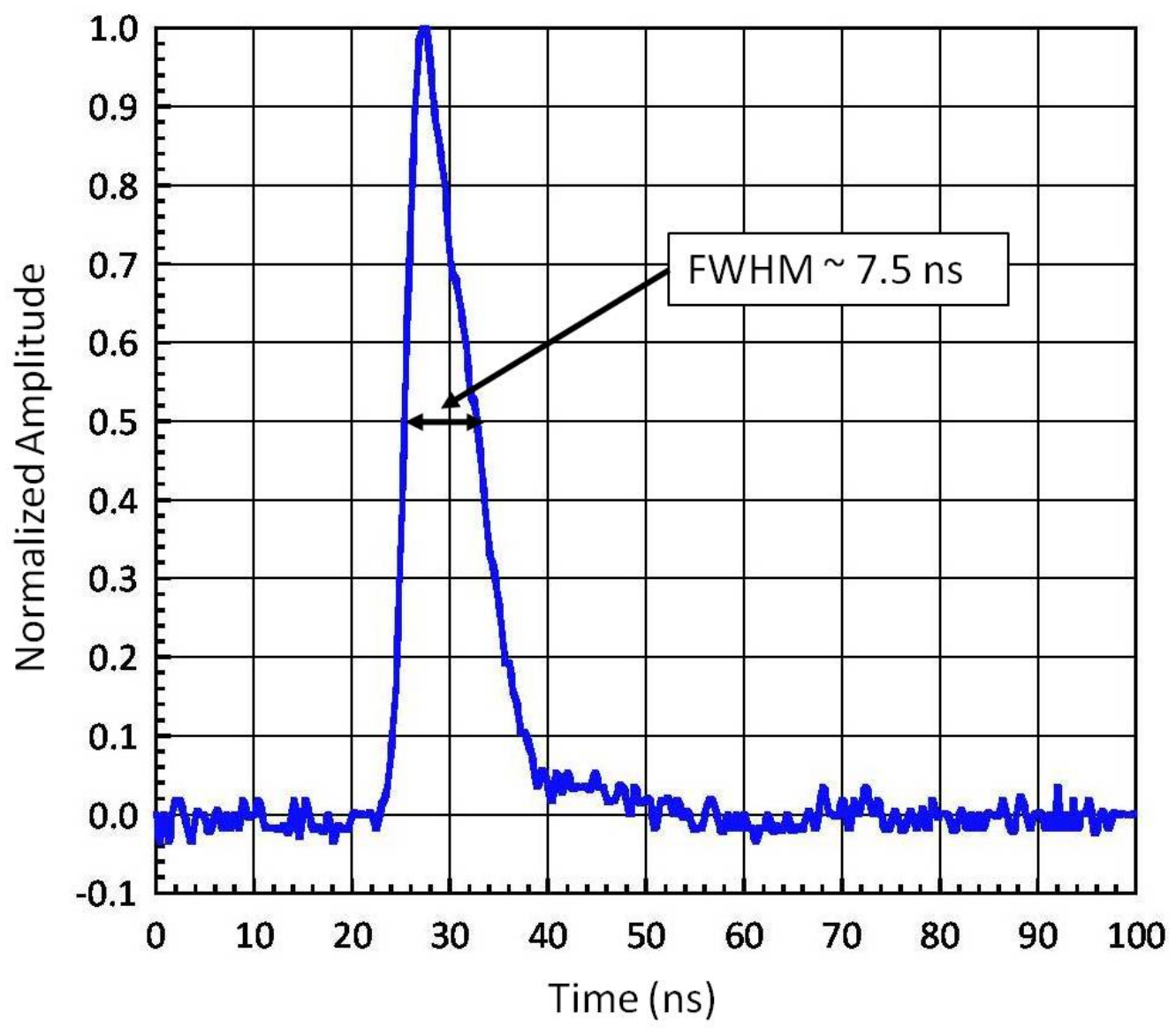

Figure 13. Time response of an nTOF detector found at the Idaho Accelerator Center (IAC). The FWHM is approximately $7.5 \mathrm{~ns}$. This was made with 50 picosecond bursts of $x$-rays. It will be convolved with a neutron impulse response later in order to include both timing and neutron impulse response information. 
The convolution of two functions $r(t)$ and $s(t)$, is denoted by:

$$
(r \otimes s)_{j} \equiv \sum_{k=-\frac{M}{2}+1}^{\frac{M}{2}} s_{j-k} r_{k}
$$

Typically $s$ is a signal or data stream, and $r$ is a response function of finite duration $\mathrm{M}$. The effect of convolution is to smear - or broaden - the signal $s(t)$ in time according to the response function $r(t)[25]$.

\section{BROADENING DUE TO TEMPERATURE AND TIME RESPONSE}

In an "Ideal Case," an nTOF detector at 809 cm (26.54 ft), location "D" in Figure 1, from a 4 keV DD fusion source would produce a FWHM according to:

$$
\text { Temp }=16578.1944 * \frac{(F W H M)^{2}}{D^{2}}
$$

where Temp is in keV, D is in $\mathrm{cm}$, and FWHM is in ns. Thus, the broadening due to temperature alone would be 12.57 ns for the parameters listed above. Then, folding in a time response of $7.5 \mathrm{~ns}$, in quadrature [26], the FWHM becomes:

$$
\begin{gathered}
F W H M=\sqrt{(\text { Temp } F W H M)^{2}+(\text { Time Response FWHM })^{2}} \\
\text { or, } F W H M=14.64 n s
\end{gathered}
$$

Broadening due to temperature and temperature plus time response is shown in Figure 14. Note how the time response broadens the signal and adds a small "tail" to the waveform. Also, the peak shifts to the right in time from 373.16 ns to 379.76 ns due to the convolution of the time response. 
Another contributor to broadening in the real world is the thickness of scintillator itself. This is shown in Table II for plastic scintillator thicknesses ranging from $0.3175 \mathrm{~cm}\left(1 / 8^{\prime \prime}\right)$ to $20.32 \mathrm{~cm}\left(8^{\prime \prime}\right)$. This data was obtained by running an "Ideal Case" i.e., a scintillator of varying thickness at a distance of $809 \mathrm{~cm}$ from a $4 \mathrm{keV}$ DD fusion source, and producing a waveform by the technique described herein. Also shown in the table is the broadening due to the convolution of the time response.

However, what role does neutron scattering play in the broadening of the detector response? It cannot be subtracted out in quadrature, since it is not a Gaussian phenomenon. Nevertheless, since it is entirely a function of how much structural and shielding material are near the detector, it would be unique in every location, and totally dependent on the local geometry. Therefore, the simplest approach would be to take the total FWHM and subtract out the FWHM due to temperature and time
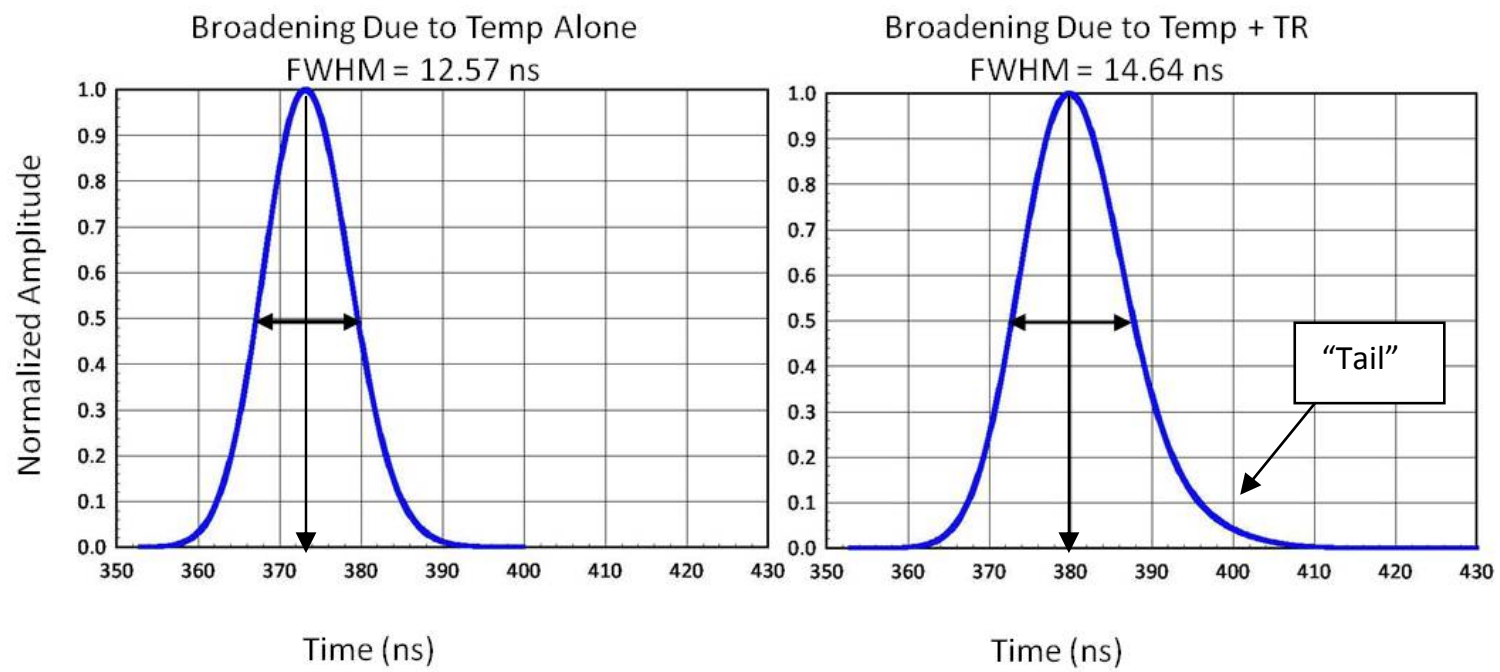

Figure 14. Broadening due to $4 \mathrm{keV}$ temperature alone (right), and a $4 \mathrm{keV}$ temperature and a time response of $7.5 \mathrm{~ns}$ (left). In the first case, the FWHM $=12.57 \mathrm{~ns}$; in the second, the FWHM $=14.64 \mathrm{~ns}$. Note that the peak shifts to the right in time from $373.16 \mathrm{~ns}$ (left) to $379.76 \mathrm{~ns}$ (right) due to the folding in of the time response, which broadens the signal, and produces a small "tail" (right). 
response; the remainder would be the broadening due to neutron scattering at that particular detector location.

Table II.

Temporal Broadening due to Scintillator Thickness and Time Response for a 4 keV DD Fusion Source placed at $809 \mathrm{~cm}^{+}$

\begin{tabular}{|c|c|c|}
\hline Thickness of Scintillator & $\begin{array}{c}\text { Broadening due to Thickness } \\
\text { (ns) }\end{array}$ & $\begin{array}{c}\text { Broadening due to Time } \\
\text { Response (ns) } \\
\text { Ave: }<2.437>\end{array}$ \\
\hline $0.3175 \mathrm{~cm}\left(1 / 8^{\prime \prime}\right)$ & ---394 \\
\hline $1.27 \mathrm{~cm}\left(1 / 2^{\prime \prime}\right)$ & 0.03 & 2.425 \\
\hline $2.54 \mathrm{~cm}\left(1^{\prime \prime}\right)$ & 0.03 & 2.43 \\
\hline $5.08 \mathrm{~cm}\left(2^{\prime \prime}\right)$ & 0.08 & 2.518 \\
\hline $7.62 \mathrm{~cm}\left(3^{\prime \prime}\right)$ & 0.33 & 2.441 \\
\hline $10.16 \mathrm{~cm}\left(4^{\prime \prime}\right)$ & 0.53 & 2.418 \\
\hline $15.24 \mathrm{~cm}\left(6^{\prime \prime}\right)$ & 0.83 & 2.425 \\
\hline $20.32 \mathrm{~cm}\left(8^{\prime \prime}\right)$ & 1.03 & 2.441 \\
\hline
\end{tabular}

${ }^{\dagger}$ Ideally, for a $4 \mathrm{keV}$ DD Fusion Source, broadening due to temperature alone is given by equation (7) above to be $12.57 \mathrm{~ns}$, and broadening due to convolution with the time response is given by equation (8) above to be $14.64 \mathrm{~ns}$.

\section{COMPARING CALCULATIONS WITH EMPIRICAL DATA}

Using the MATLAB code written for convolution, the time response was convolved ("folded in") with the post-processor output and compared with empirical data. In Figure 15, the calculated detector response is compared to shot z1217, with the machine in a configuration as shown in Figure 3. The plots are area normalized. The neutron source used in the MCNP-PoliMi model was a 4 keV DD Fusion Source. 


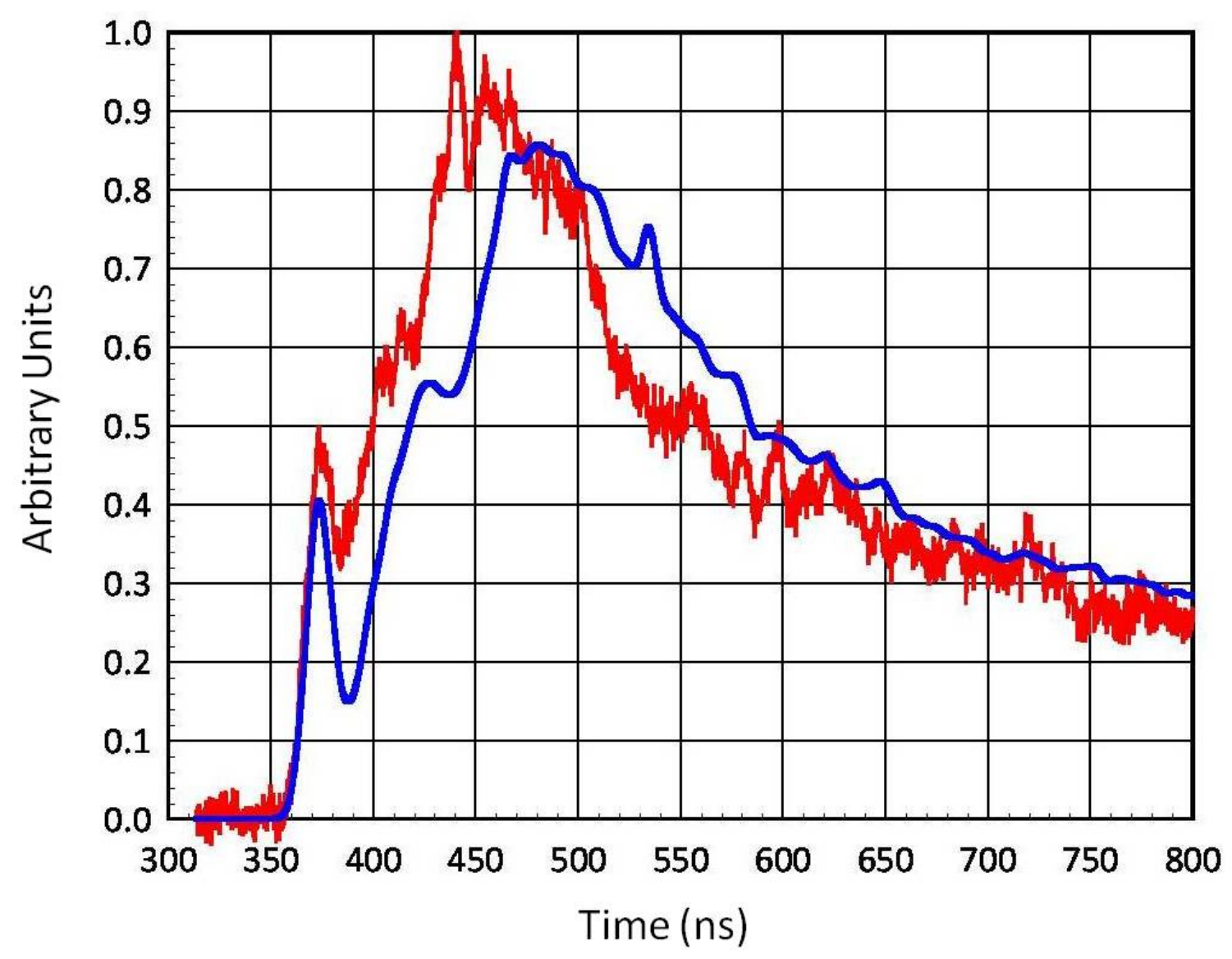

Figure 15. Area normalized comparison between shot z1217 without TIVAR 1000 Collimator (red) and MCNP-PoliMi model with folded-in time response for a $4 \mathrm{keV}$ DD fusion source (blue) for a detector located at " $D$ " in Figure 1. The same features can be observed: a primary neutron peak followed by a very large, second scattering peak.

A close up of the neutron peak from shot z1217 at detector location " $D$ " in Figure 1 is compared to the model in Figure 16 below. The model shows a full width at half maxima of $16.38 \mathrm{~ns}$. A FWHM cannot be extracted from the data due to the large, second scattering peak. Both plots are area normalized.

For the nTOF detector located at " $\mathrm{C}$ " in Figure 1, an MCNP-PoliMi model produced a neutron detector response and the detector time response was folded in as described above. A plot of the MCNP-PoliMi model compared with the actual empirical 
data for the nTOF detector located at " $\mathrm{C}$ " for shot z1217 is shown in Figure 17. As can

be seen, there is better separation between the primary neutron peak and the secondary scattering peak compared to Figure 15 . Also, the neutron peak has a greater amplitude relative to the scattering peak. Both plots are area normalized.

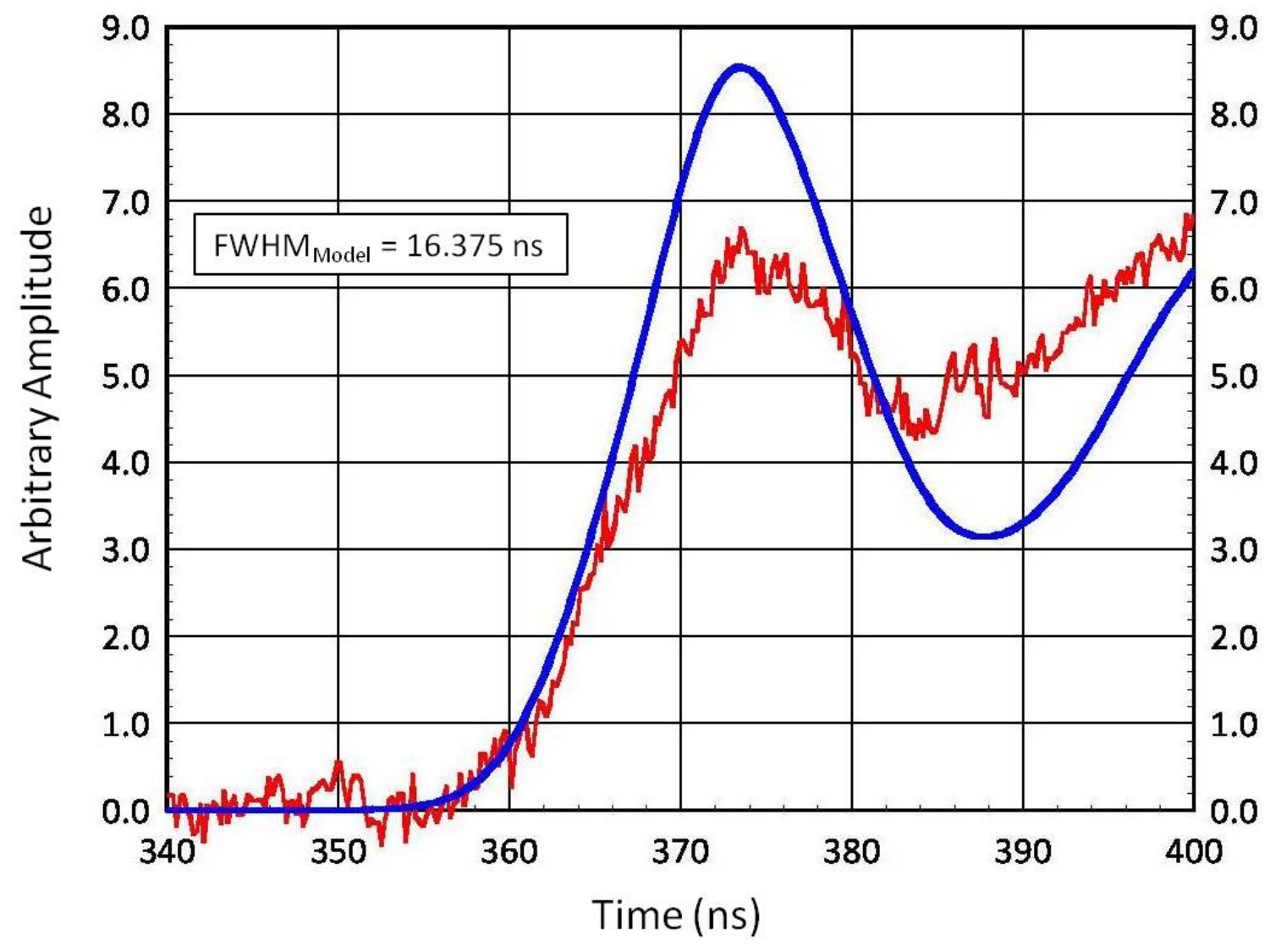

Figure 16. Close-up of the primary neutron peak in Figure 15, for the bottom nTOF detector located at "D" in Figure 1 . The model was run at a temperature of $4 \mathrm{keV}$, and its full width at half maxima is $16.38 \mathrm{~ns}$. A FWHM from the data cannot be extracted due to the large, second scattering peak following the neutron peak.

A close up of the neutron peak for shot z1217 located at " $C$ " in Figure 1 is compared to the model in Figure 18. The FWHM of the data is $14.89 \mathrm{~ns}$, while the FWHM of the model is $15.11 \mathrm{ns.} \mathrm{Both} \mathrm{plots} \mathrm{are} \mathrm{area} \mathrm{normalized.}$ 


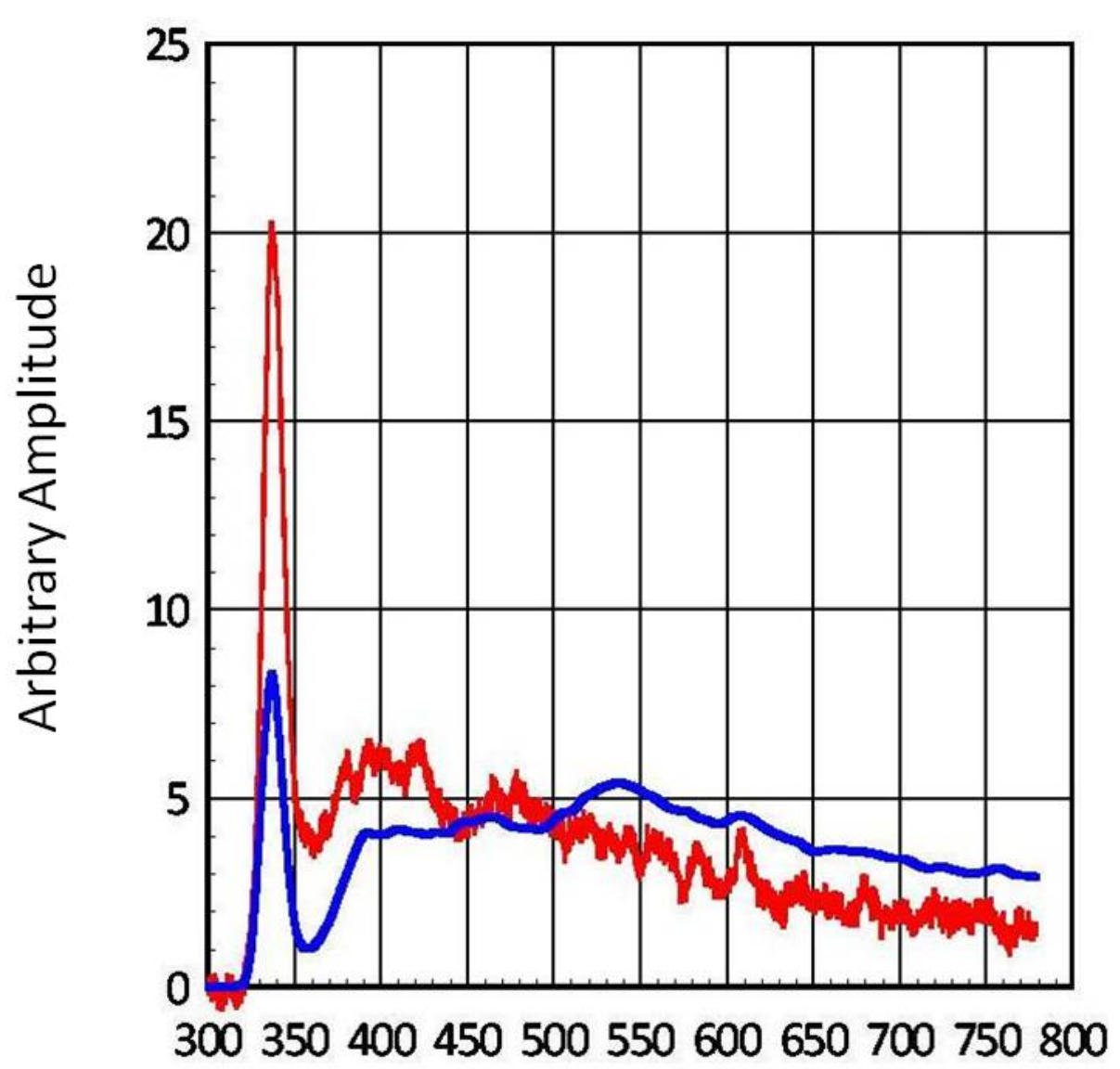

Time (ns)

Figure 17. Area normalized comparison between shot z1217 without Tivar Collimator (red) and MCNP-PoliMi model with folded-in time response for a $4 \mathrm{keV}$ DD fusion source (blue) for a detector located at " $\mathrm{C}$ " in Figure 1. There is better separation between the primary neutron peak and the second scattering peak than shown in Figure 15. Also, the neutron peak has a greater amplitude relative to the scattering peak than that shown in Figure 15.

To reduce the second scattering peak seen in Figure 17 for the detector located at " $C$ " (in Figure 1) and to lessen the second scattering tail seen in Figure 15 for the other detector located at "D" (in Figure 1), a collimator made of UHMW TIVAR 1000 was built and placed under TCC as seen in Figure 19. This material was chosen over regular polyethylene because it does not outgas under vacuum [27]; (see also Appendix F). The collimator was $25.4 \mathrm{~cm}$ (10 in) long and had a tungsten insert on axis serving as a 
gamma ray collimator for the intense bremsstrahlung background. The length of 25.4 $\mathrm{cm}$ (10 in) was chosen to be manageable to install; also MCNPX calculations showed that that length would attenuate DD neutrons by approximately a factor of 1000 .

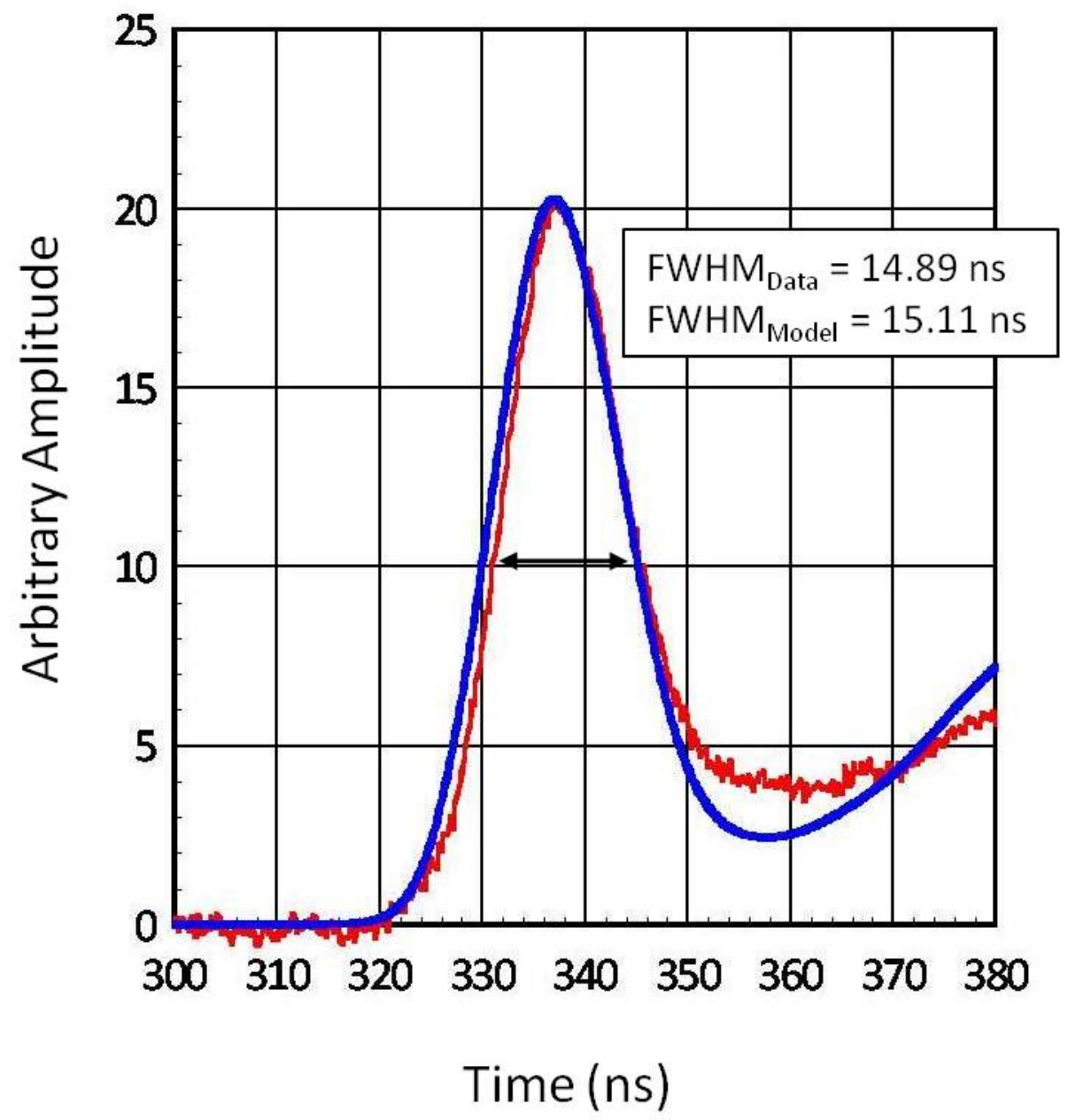

Figure 18. Close-up of the primary neutron peak in Figure 17, for the top nTOF detector located at " $\mathrm{C}$ " in Figure 1. The model was run at a temperature of $4 \mathrm{keV}$, and its full width at half maxima is $15.11 \mathrm{~ns}$, while the data from shot z1217 has a full width at half maxima of 14.89 ns. Both plots are area normalized.

The exit aperture on the collimator was $7.62 \mathrm{~cm}(3 \mathrm{in})$. When projected downward, the collimator would cast a "shadow" all the way down to the basement floor, $880.76 \mathrm{~cm}(28.9 \mathrm{ft})$ below the pinch (TCC). This is shown in Figure 20. With the pig being 3 degrees off-axis, this made both nTOF detectors just fit inside the collimator 
"cone," as shown in Figure 21. However, it was hoped the collimator would reduce neutron scattering off the elevator floor that may have been contributing to the second scattering peak for the nTOF detector located at " $D$ " in Figure 1 (i.e., the detector closest to the floor) as seen in Figure 6.

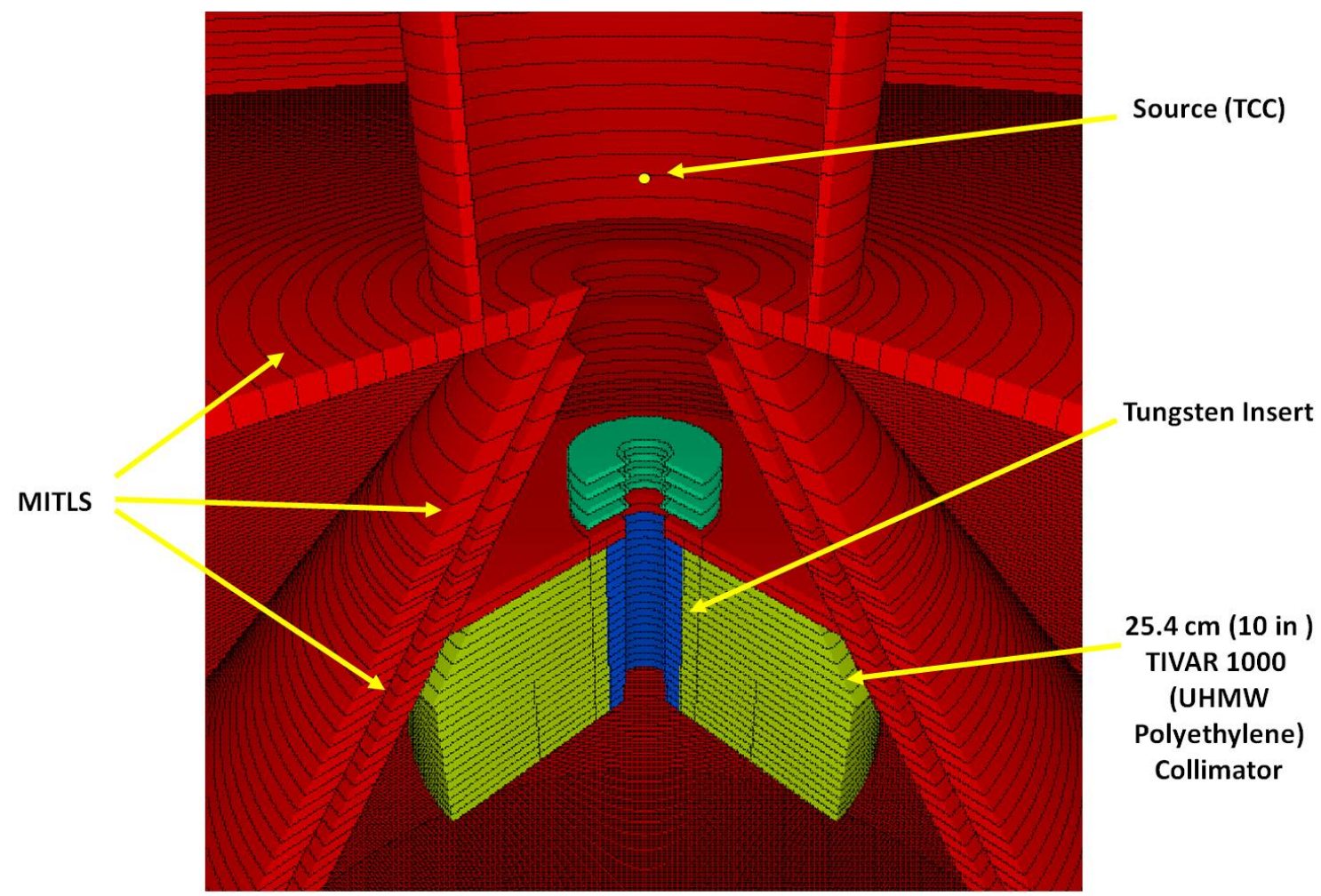

Figure 19. A closer view of the UHMW TIVAR 1000 collimator incorporated into the machine on neutron producing shots in order to help "clean up" neutron signals. MCNPX calculations showed that Its length of $25.4 \mathrm{~cm}$ (10 in) would attenuate DD neutrons by approximately a factor of 1000 . The tungsten insert is serving as a gamma ray collimator.

A plot of an MCNP-PoliMi model with the collimator in place, and the detector response convolved with the calculated signal (blue) compared to shot z1549 when the collimator was fielded on the machine (red) is shown in Figure 22. As can be seen, use of the collimator greatly reduced the second scattering peak and produced a much 
greater amplitude of the primary neutron signal relative to the second peak as compared to Figure 15.

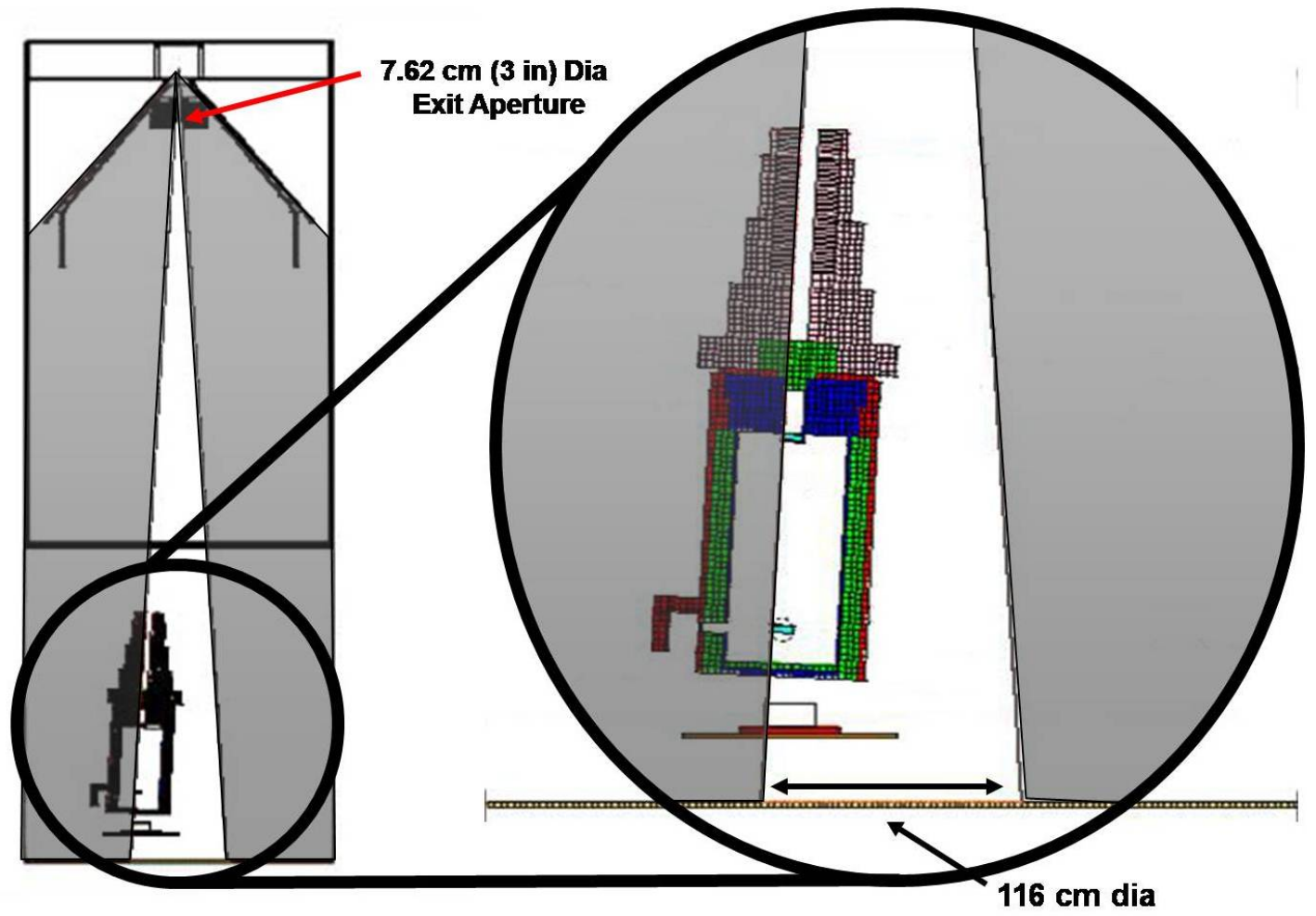

Figure 20. "Shadow" of TIVAR 1000 Collimator. With a $7.62 \mathrm{~cm}$ (3 in) diameter exit aperture growing to a $116 \mathrm{~cm}$ (45.7 in) diameter spread at the basement floor $880.76 \mathrm{~cm}$ $(28.9 \mathrm{ft})$ below TCC.

A close up of the neutron peak for shot z1549 located at " $D$ " in Figure 1 is compared to the model in Figure 23. The FWHM of the data is $12.75 \mathrm{~ns}$, while the FWHM of the model is $11.84 \mathrm{~ns}$. The model was run at a temperature of $2 \mathrm{keV}$ for a DD fusion neutron source. Both plots are area normalized.

For the top nTOF detector located at "C" in Figure 1, a plot of its MCNP-PoliMi model with folded in time response (blue) is compared with shot z1549 for a fielded detector in the same location (red) in Figure 24. As can be seen, the effect of the collimator was to virtually eliminate the second scattering tail that was seen in Figure 17 
(before the collimator was added). A close-up of the neutron scattering peak is shown in Figure 25. The full width at half maxima for the data is $12.70 \mathrm{~ns}$, while the full width at half maxima for the model is $11.20 \mathrm{~ns}$. Of note is that the temperature of the actual experiment is unknown, while the model was run with a $2 \mathrm{keV} D D$ fusion neutron source.

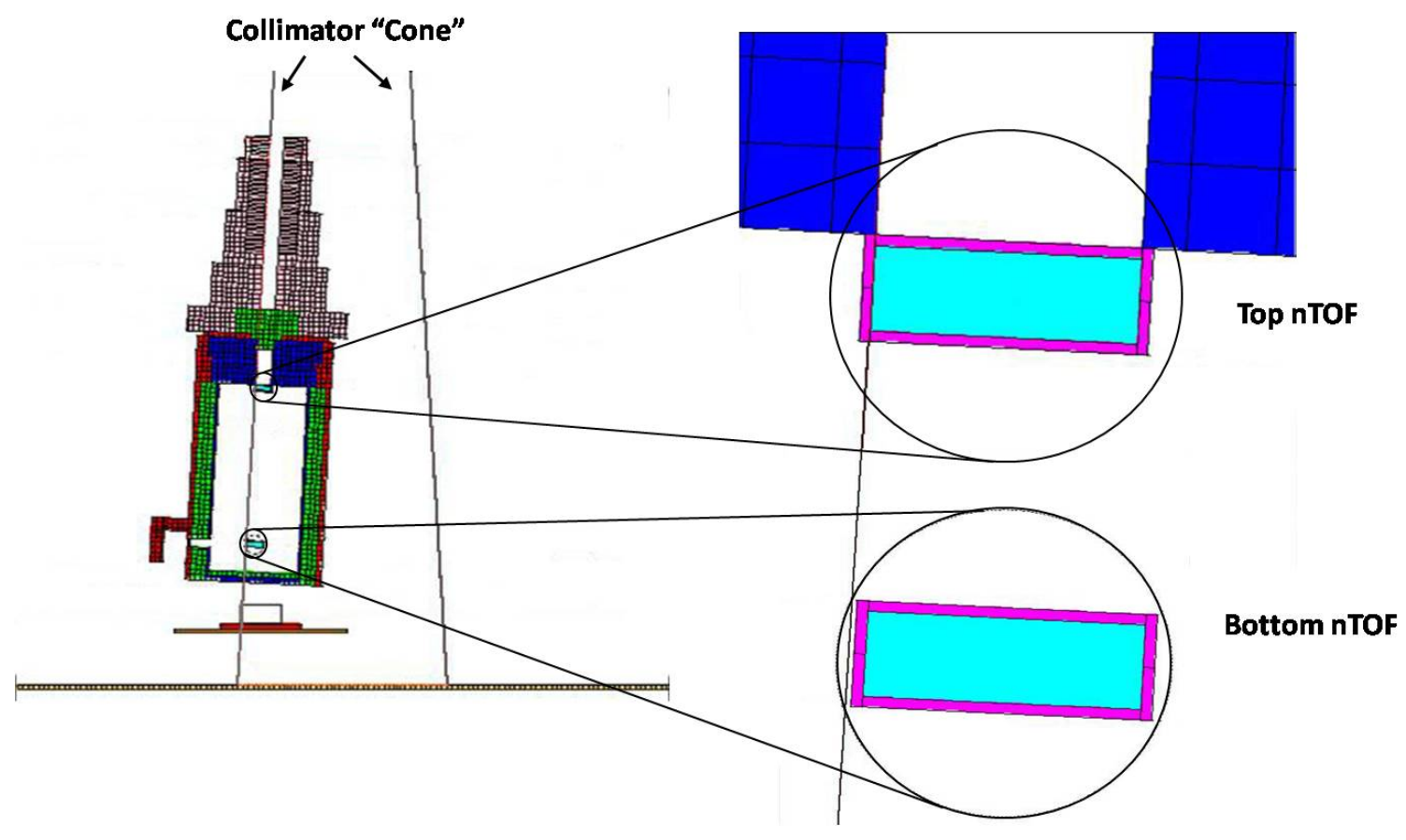

Figure 21. With the pig aligned 3 degrees off-axis, the collimator "cone" just encompassed both detectors. However, it was still hoped that the addition of the collimator would reduce neutron scattering off the elevator floor that may be contributing to the second scattering peak seen in Figure 15.

As can be seen from Figures 23 and 25 , collimation is essential to produce "cleaner" neutron signals. It will be shown later that it does indeed reduce shallowangle scattering in the neutron peak, and it helps eliminate the bulk of scattering arriving later in time. As shown by the "shadow" in Figure 20, neutrons removed early 
in time by the collimator near the source therefore cannot arrive later in time at the detectors by scattering.

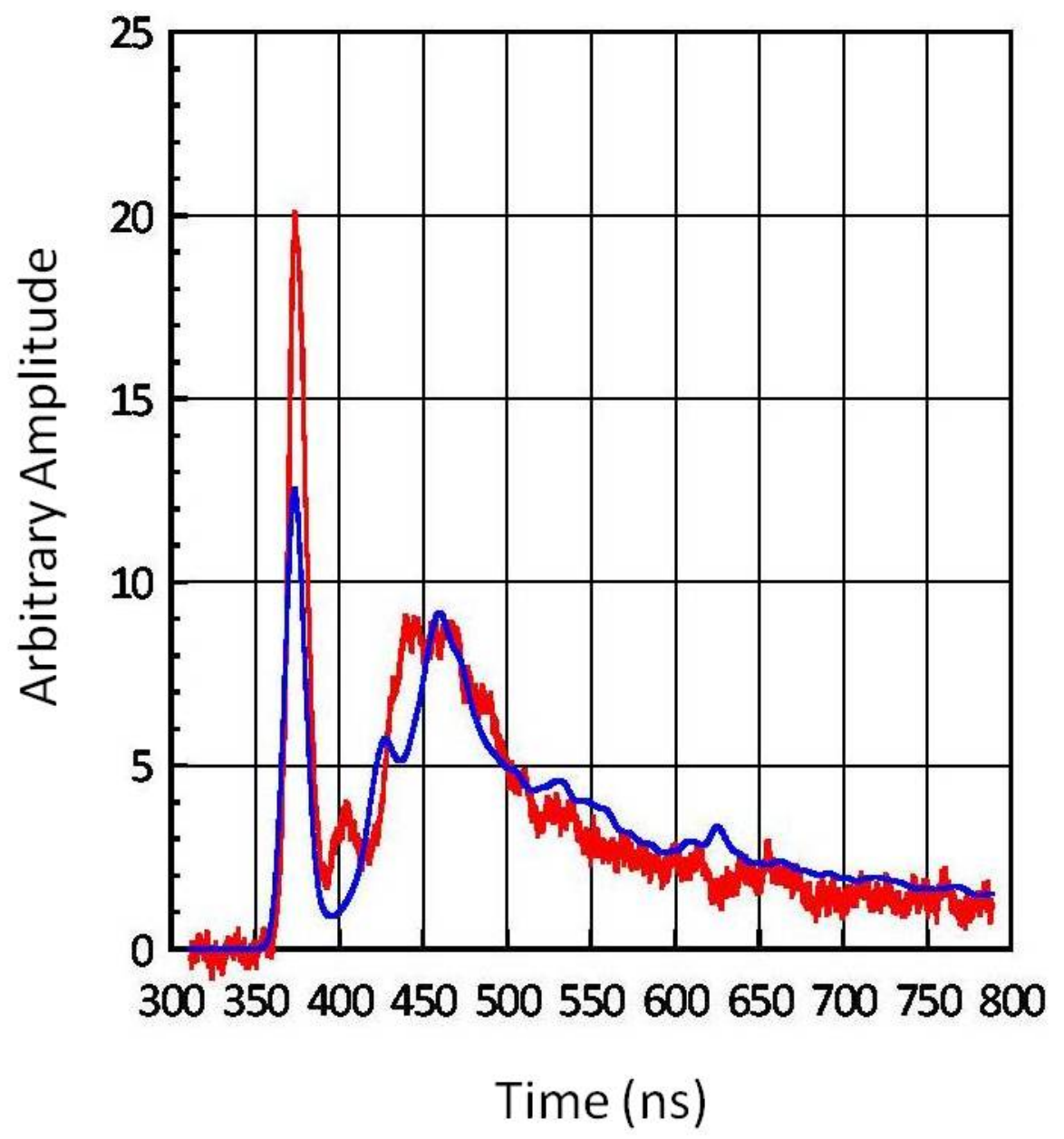

Figure 22. Area normalized comparison of shot $z 1549$ (red) for detector at location " $D$ " in Figure 1 with TIVAR 1000 Collimator in place and MCNP-PoliMi model with folded in time response of $7.5 \mathrm{~ns}$ (blue). Use of the collimator greatly reduced the second scattering peak and produced a much greater amplitude of the primary neutron signal compared to the second peak as compared to Figure 15. 


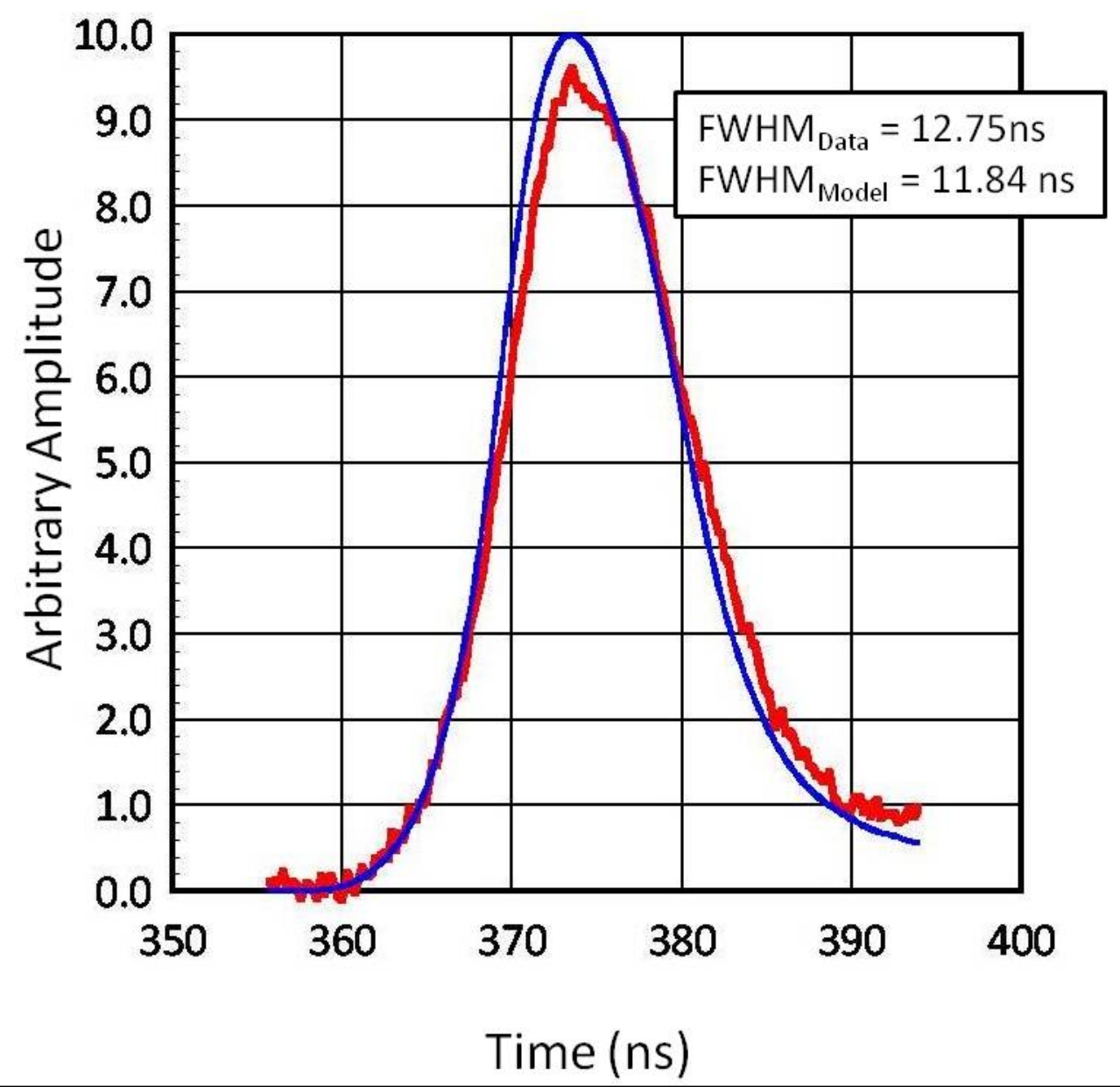

Figure 23. Close-up of primary neutron peak in Figure 22, for the bottom nTOF detector located at " $D$ " in Figure 1. The full width at half maxima of the data is $12.75 \mathrm{~ns}$, while the full width at half maxima for the model is 11.84 ns. The model was run with a 2 keV DD fusion neutron source. 


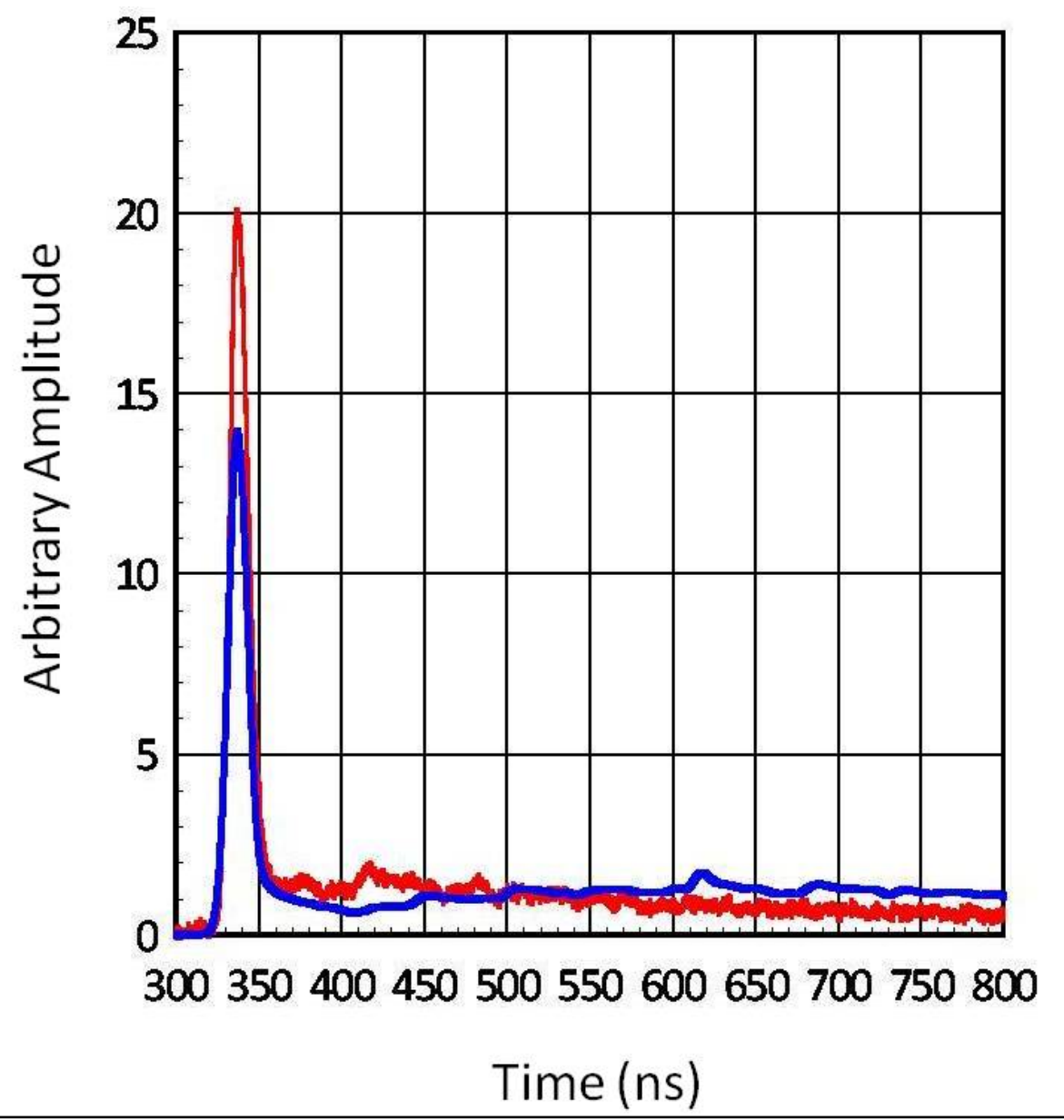

Figure 24. Area normalized comparison of shot $z 1549$ (red) for detector at location " $C$ " in Figure 1 with Tivar 1000 Collimator in place and MCNP-PoliMi model with folded in time response of $7.5 \mathrm{~ns}$ (blue). The effect of the collimator was to virtually eliminate the second scattering tail that was seen in Figure 17. 


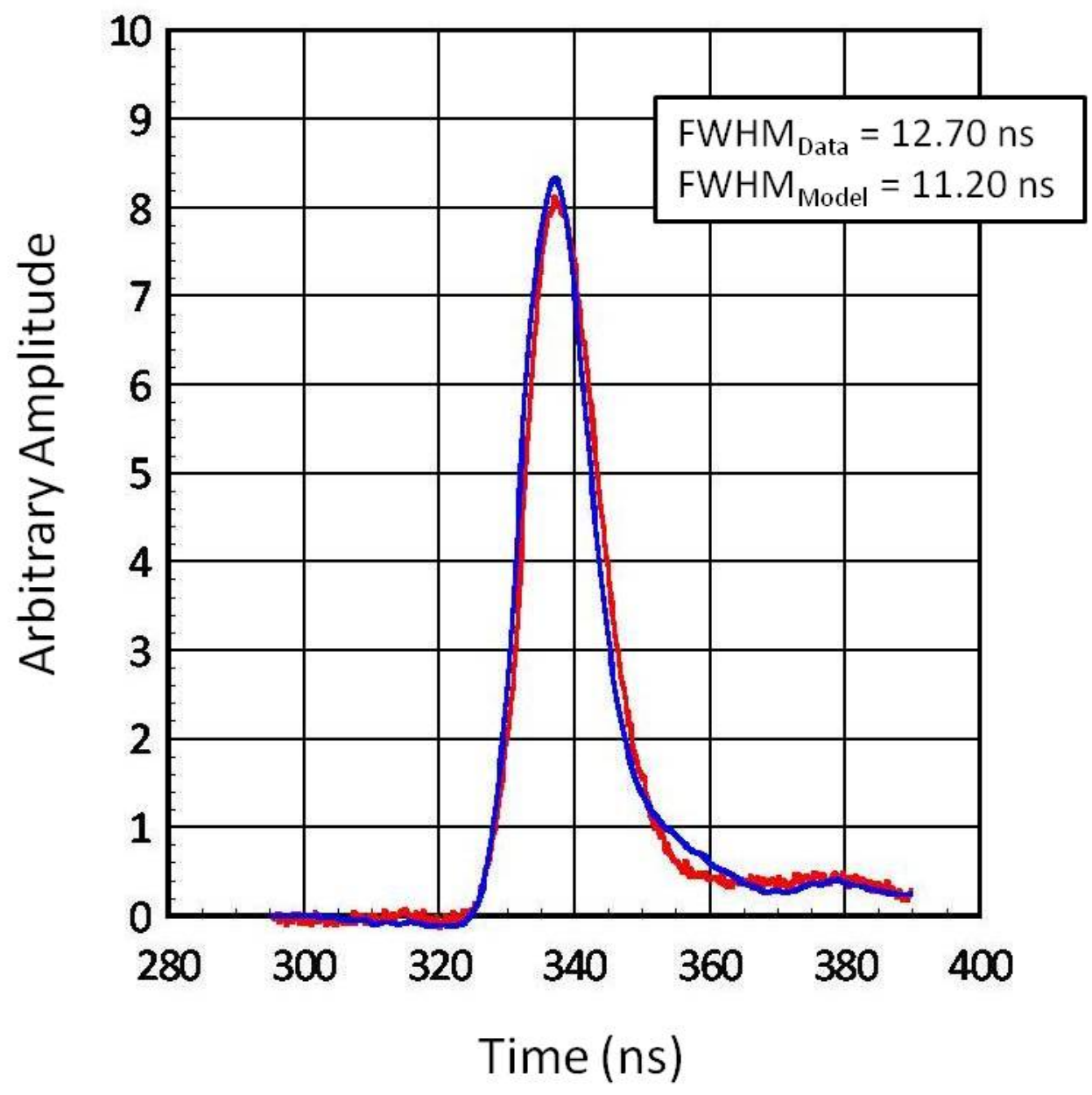

Figure 25. Close-up of primary neutron peak in Figure 21, for the top nTOF detector located at " $\mathrm{C}$ " in Figure 1 with the TIVAR 1000 Collimator in place. The full width at half maxima for the data is $12.70 \mathrm{~ns}$, while the full width at half maxima for the model is 11.20. The model was run with a temperature of $2 \mathrm{keV}$ DD fusion neutron source. 


\section{CHAPTER 7}

\section{DECONVOLVING THE TIME RESPONSE FROM THE SIMULATED DATA}

It was shown above that a detector's intrinsic time response will broaden the detector's signal - in effect, it "smears" the data in time to some degree. To further analyze the data, however, this time response must be deconvolved - or "unfolded" from the data. Deconvolution is a process of undoing the smearing in the data that has occurred due to the influence of a known response function. The equation for deconvolution is the same as that for convolution, namely equation (6), except that now the left hand side is taken to be known, and (6) is to be considered as a set of $\mathrm{N}$ linear equations for the unknown quantities $s_{j}$. This can be accomplished using Fast Fourier Transforms. First, the transform of the signal (which is convolved with the response function) is taken. Next the transform of the response function is taken. The transform of the signal is now divided by the transform of the response - this gives the transform of the deconvolved signal. The last step is to take the inverse FFT to finally obtain the raw signal.

To make sure the process was correct, the time response in Figure 13 was deconvolved from the MCNP-PoliMi model with folded-in time response for a 4 keV DD fusion source (blue), shown in Figure 15. After deconvolving, the result was compared with the smoothed calculated data shown in Figure 12 (red) and will be discussed below. A set of codes from Numerical Recipes was used to accomplish this [25]. 
Prior to attempting Fast Fourier Transforms, the signal and time response have to be "prepared." The number of points in the signal must be an integer power of 2, and "zero-padded" (i.e., extended with zeros) at its extreme end in time. The amount of zero-padding at the end of the data must equal the number of data points in region " $\mathrm{A}$ " or " $\mathrm{B}$ " in Figure 27 - whichever is larger. This is shown in Figure 26 below.

The time response has to be placed in "wrap-around order," meaning the data is considered as being wrapped around a cylinder with the ends touching - this means

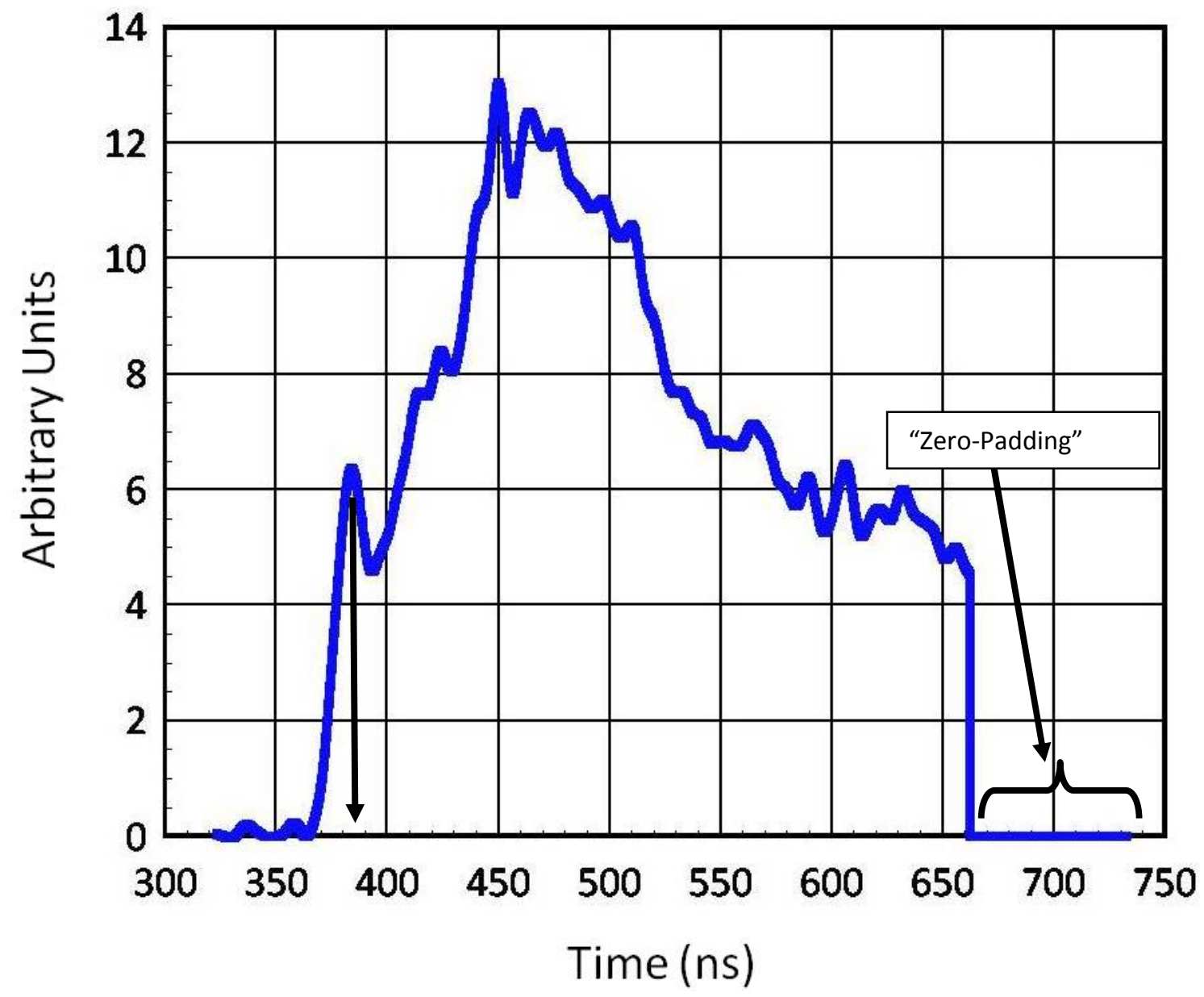

Figure 26. Preparation for the signal prior to taking Fast Fourier Transforms. This is the empirical data from shot z1217. The total number of points in the signal must be an integer power of 2, and "zero-padded" - or extended with zeros at its extreme end in time. The amount of zero padding must equal the number of points in region " $A$ " or " $B$ " in Figure 27 - whichever is larger. (Note that the neutron peak is at 383.13 ns - see arrow.) 


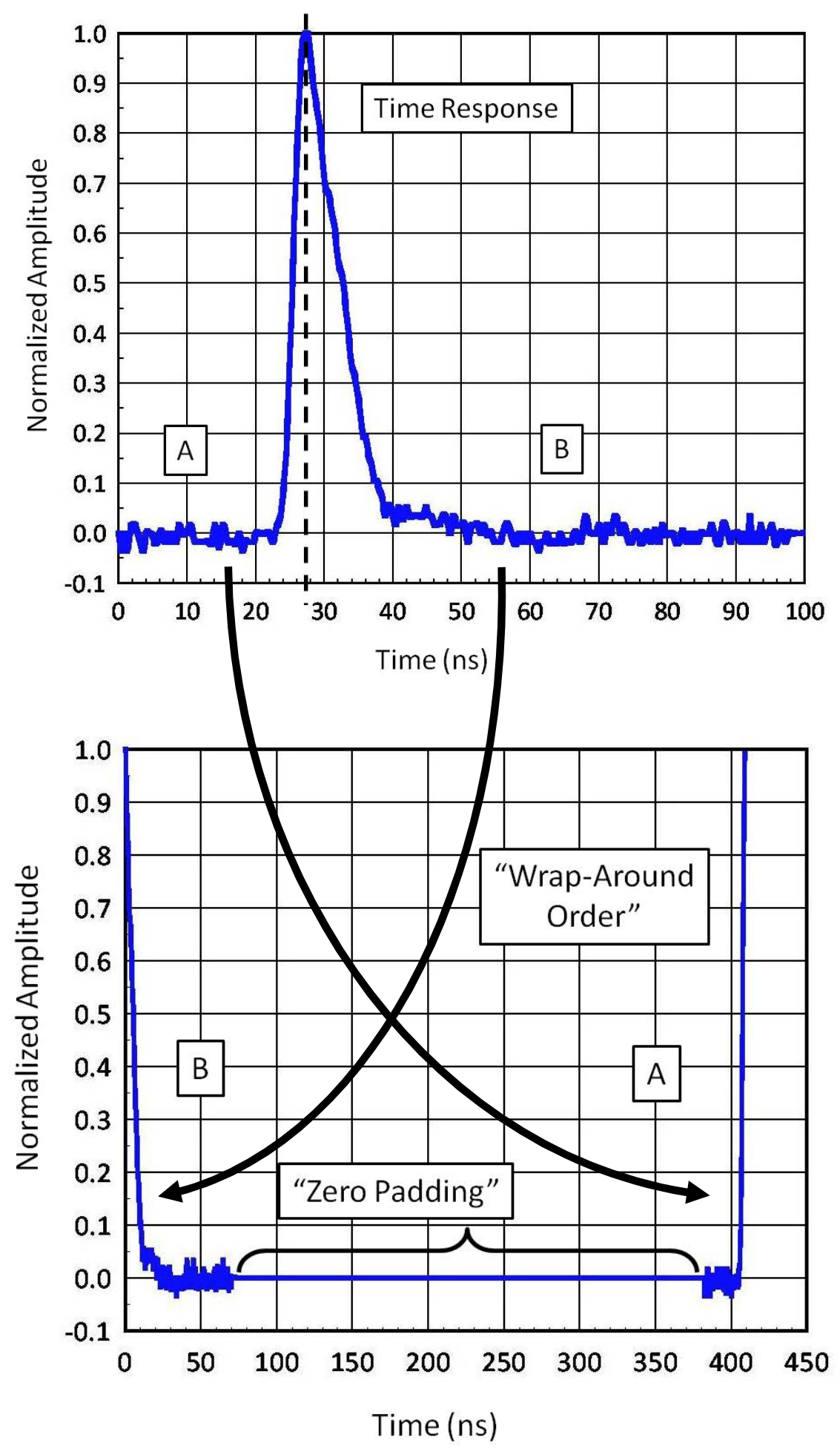

Figure 27. "Wrap-Around Order." First the time response (above, and the same as shown in Figure 13), is cut in half at its peak. Then each side of the time response is flipped left-for-right (see arrows), then "zero-padded" in the middle. The total number of points, $\mathrm{M}$, can be any odd integer less than or equal to $\mathrm{N}$, the number of points in the data, which must be an integer power of 2 . 
that a large center section in the middle of the data, is zero, with nonzero values clustered at the two extreme ends. This is shown in Figure 27.

Once the data and the response function have been prepared properly, the deconvolution can be accomplished by Fast Fourier Transforms. A plot of the smoothed, calculated data (blue) is compared with the deconvolved fit (red), and the smoothed, deconvolved fit (green) is shown in Figure 28. The blue and green traces fall neatly on top of each other, showing good agreement.

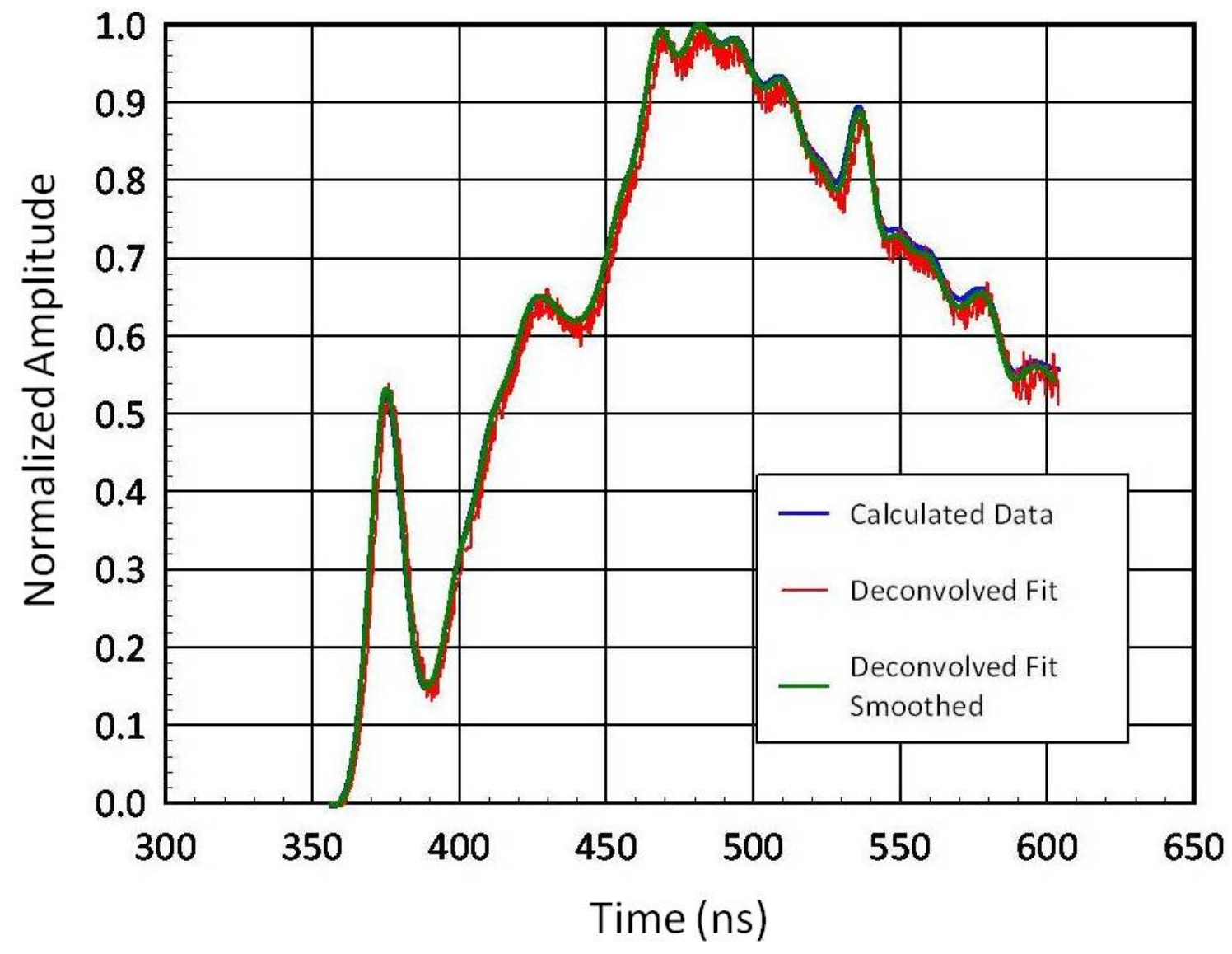

Figure 28. The smoothed calculated data (blue, also shown in Figure 12 with a red trace) is compared to the deconvolved fit using Fast Fourier Transforms (red). The deconvolved fit is smoothed (green) and can be seen laying on top of the calculated data, showing good agreement. Both blue and green traces have been smoothed with the Savitsky-Golay smoothing filter. 


\section{CHAPTER 8}

\section{CONVOLVING A NEUTRON IMPULSE RESPONSE WITH THE KNOWN TIME RESPONSE}

Using the techniques described herein, a monoenergetic source of neutrons of $2.45 \mathrm{MeV}$ were run with a $2.54 \mathrm{~cm}$ (1 inch) scintillator placed at $809 \mathrm{~cm}$. The resulting waveform can be described as the calculated neutron impulse response for the scintillator, and is shown in Figure 29 below. The shape of the waveform from $374.51 \mathrm{~ns}$ (where it begins) down to $375.71 \mathrm{~ns}$ (both indicated by arrows) is a time span of $1.2 \mathrm{~ns}$.

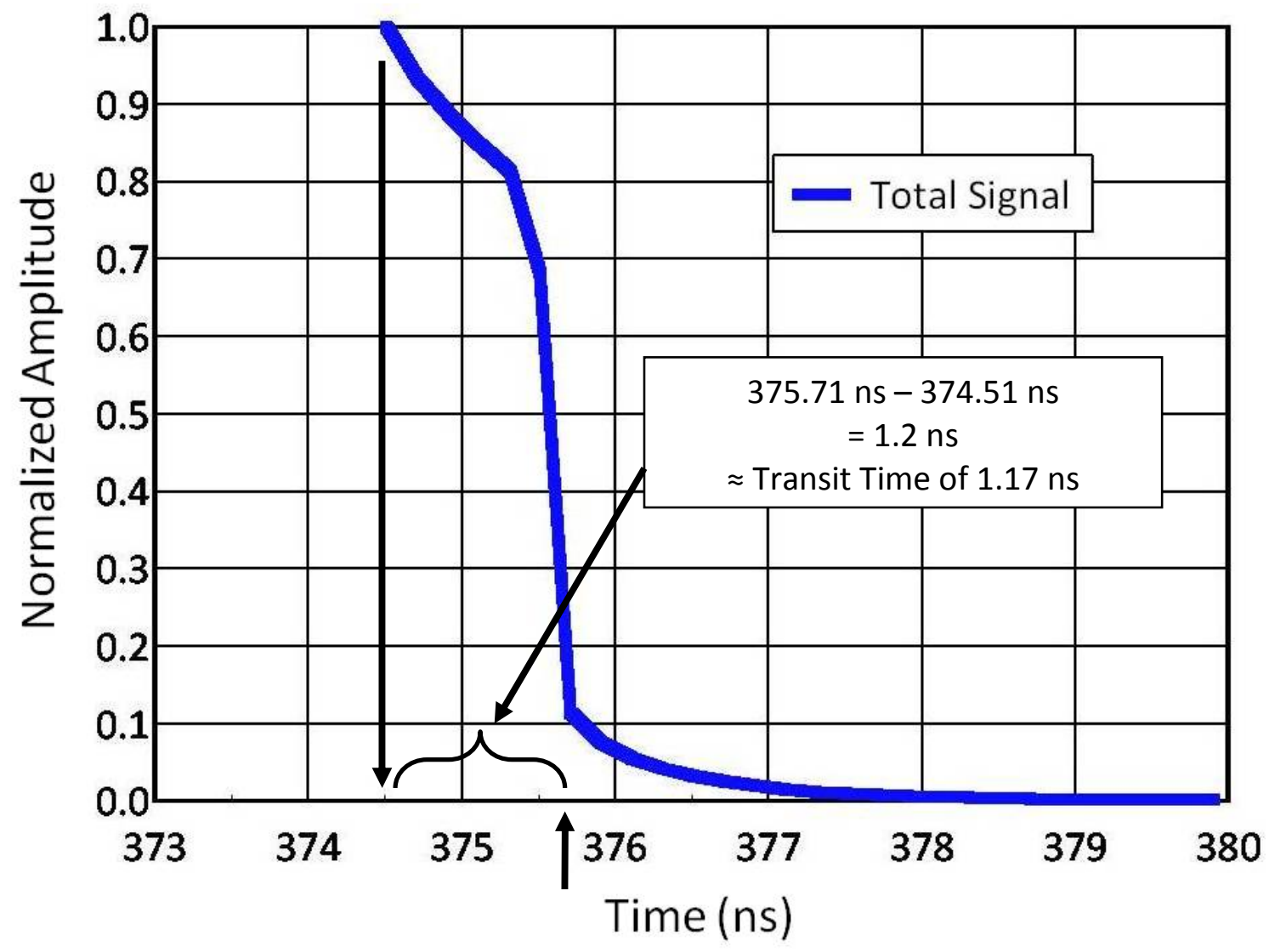

Figure 29. The neutron impulse response for a $2.54 \mathrm{~cm}$ (1 inch) scintillator placed $809 \mathrm{~cm}$ from a monoenergetic source of $2.45 \mathrm{MeV}$ DD neutrons. The region in time indicated by the bracket is $1.2 \mathrm{~ns}$, which is due to the transit time of a $2.45 \mathrm{MeV}$ neutron traversing the scintillator. The curved portion of the waveform which follows is due explicitly to secondary scattering in the scintillator, and is described in Figure 30. 
This value of $1.2 \mathrm{~ns}$ correlates to the transit time of a $2.45 \mathrm{MeV}$ neutron passing through a $2.54 \mathrm{~cm}$ (1 inch) scintillator, and can be found by:

$$
K E=\frac{1}{2} m v^{2}
$$

Substituting $2.45 \mathrm{MeV}$ for $K E$, letting $\mathrm{m}=\mathrm{m}_{0}$, and multiplying by $\mathrm{c}^{2} / \mathrm{c}^{2}$ gives:

$$
\begin{gathered}
2.45 \mathrm{MeV}=\frac{1}{2} \frac{v^{2}}{c^{2}} m_{0} c^{2} \\
\text { Solving for } \mathrm{v} / \mathrm{c}: \\
\frac{v}{c}=\sqrt{\frac{(2)(2.45 \mathrm{MeV})}{m_{0} c^{2}}}
\end{gathered}
$$

Letting $c=2.9979 \mathrm{E} 10 \mathrm{~cm} / \mathrm{sec}$, and $\mathrm{m}_{0} \mathrm{c}^{2}=939.5653 \mathrm{MeV}$ for a neutron and solving for $\mathrm{v}$ :

$$
v=\sqrt{\frac{(2)(2.45 \mathrm{MeV})}{939.5653 \mathrm{MeV}}}\left(2.9979 E 10 \frac{\mathrm{cm}}{\mathrm{sec}}\right)
$$

$v$ becomes:

$$
v=2.166 E 09 \frac{\mathrm{cm}}{\mathrm{sec}}\left(\frac{1 \mathrm{sec}}{10^{9} \mathrm{~ns}}\right)
$$

Thus, the velocity of a $2.45 \mathrm{MeV}$ neutron is:

$$
v=2.166 \mathrm{~cm} / \mathrm{ns}
$$

And the transit time through a $2.54 \mathrm{~cm}$ (1 inch) thick scintillator becomes:

$$
\text { Transit Time }=\frac{2.54 \mathrm{~cm}}{2.166 \mathrm{~cm} / \mathrm{ns}}
$$




\section{Transit Time $=1.17 n s$}

\section{PRIMARY AND SECONDARY NEUTRON SCATTERING}

One of the versatile features of the collision data output table (Table I), is that it contains so much germane information. As noted in this work, the incident particle, target nucleus, energy deposited and time of the event were used to model the nTOF detector response. Other information in the table includes the number of scatterings which have occurred. Using this information, Figure 29 can be further analyzed in terms of primary and secondary neutron scattering. In this context, primary scattering refers to the number of scatterings in Table I to be equal to one, and secondary scattering refers to the number of scatterings in Table I to be greater than one. The postprocessing code was easily modified to plot the light output due only to primary scattering in one case (i.e., number of scatterings $=1$ ), and only secondary scattering (i.e., number of scatterings $>1$ ) in the other. The result is shown in Figure 30 . The total signal (blue) is the same as that shown in Figure 29. The green, however, shows only the light output produced by primary scattering, and the red shows only the light output produced by secondary scattering. If both signals are summed (red and green), they equal the total signal (blue). In the span of transit time shown in Figure 30 of $1.2 \mathrm{~ns}$, $85.6 \%$ of light output is due to primary scattering, and $14.4 \%$ of light output is due to secondary scattering (this was done by summing amplitudes of the total signal, primary and secondary scattering and determining the contribution of each). Upon arriving at 
the scintillator, a neutron may collide with a hydrogen atom on the front face of the scintillator (in which case, the transit time is much less than $1.2 \mathrm{~ns}$ ), or it may traverse through the scintillator and collide with a hydrogen atom in the middle (in which case, the transit time would be $1.2 \mathrm{~ns} / 2=0.6 \mathrm{~ns})$, or it may interact at the back face of the scintillator (in which case, the transit time would be $1.2 \mathrm{~ns}$ ). Of note in Figure 30 is that after 375.71 ns the light output produced is due to secondary scattering only, since the green trace (primary scattering) drops to zero and the red trace (secondary scattering) lies on top of the blue trace (i.e., the total signal).

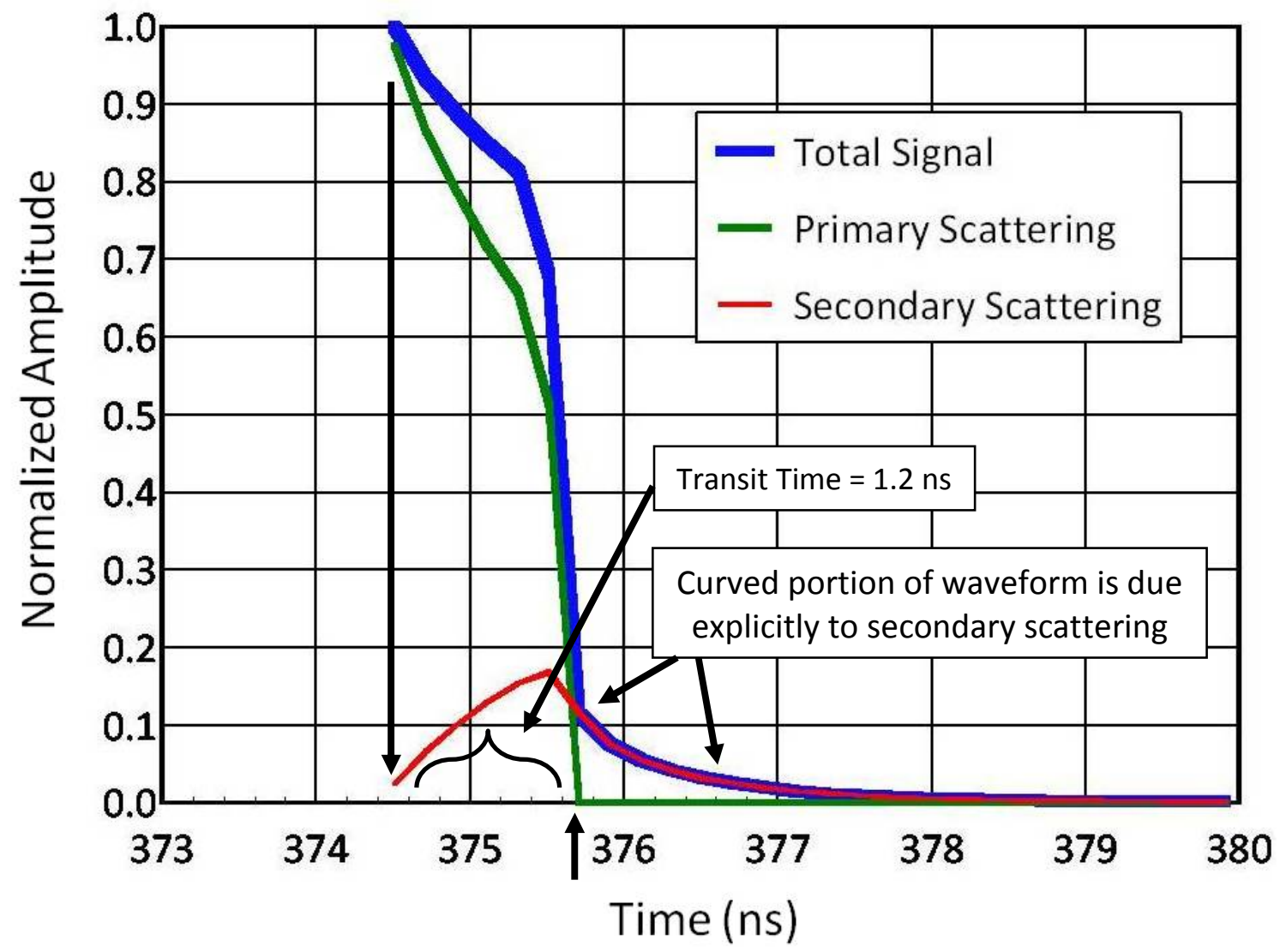

Figure 30. The neutron impulse response divided up into its component parts: primary and secondary scattering. In the span of the transit time of $1.2 \mathrm{~ns}, 85.6 \%$ of the light output is produced by primary scattering, while $14.4 \%$ of the light output is produced by secondary scattering. After $1.2 \mathrm{~ns}$ of transit time, the green trace (primary scattering) drops to zero and all light output produced is due to secondary scattering only. 
To analyze the data as correctly as possible, it was then necessary to convolve the neutron impulse response (Figure 29) with the time response (Figure 13). The reason to include both these waveforms is that they each contain information necessary for a "total" signal. Figure 13, the time response, was found by pulsing x-rays with a 50 picosecond width into the nTOF detector at the Idaho Accelerator Center (IAC) using their $15 \mathrm{MeV}$ short pulse Linac [24]; (see also Appendix E). This provided timing information due to impinging x-rays, but it did not provide any neutron impulse information. Therefore, the monoenergetic $2.45 \mathrm{MeV}$ neutron impulse response (Figure 29 ) is necessary to be included, which is why it was convolved with the time response and shown in Figure 31 below.

The FWHM of 7.607 ns in Figure 31 compares favorably with finding it analytically in quadrature:

$$
F W H M=\sqrt{(7.5 n s)^{2}+(1.2 n s)^{2}}
$$

The full width at half maxima of the monoenergetic neutron impulse response convolved with the time response becomes:

$$
F W H M=7.60 n s
$$

Once the neutron impulse response was convolved with the time response, it was then necessary to find the time delay -- i.e., the delay from neutrons impinging on the face of the scintillator to the electronic signal coming out of the base of the photomultiplier tube. This consists of three components: (1) the average transit time of a $2.45 \mathrm{MeV}$ neutron through a $2.54 \mathrm{~cm}$ (1 inch) scintillator (which is found from the value above); (2) the transit time of light produced in the scintillator that travels through 


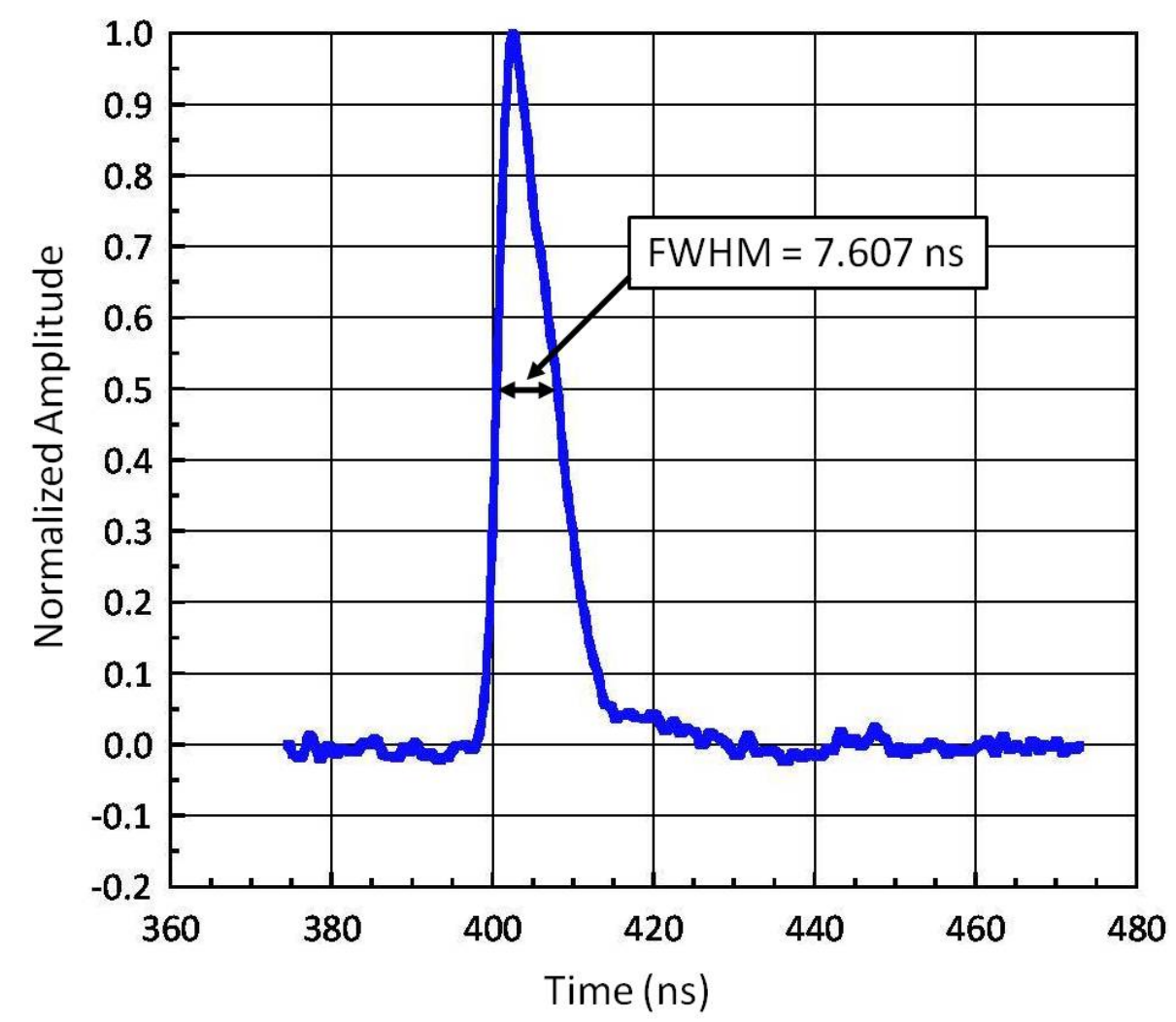

Figure 31. The convolution of the neutron impulse response (Figure 29) with the time response (Figure 13) found at the Idaho Accelerator Center (IAC). Together, both provide timing and neutron impulse response information necessary to be deconvolved out of the real data.

the light guide; and (3), the transit time through the photomultiplier tube and base, which has been measured by National Security Technologies (NSTec) [28]. A schematic of this is shown in Figure 32.

As mentioned, the transit time of a $2.45 \mathrm{MeV}$ neutron through a $2.54 \mathrm{~cm}$ (1 inch) scintillator is $1.2 \mathrm{~ns}$; thus, the average is half that value, since a neutron can interact on the front face or it can interact on the back face - therefore, it is taken to be $0.6 \mathrm{~ns}$. The transit time of the light that is produced and travels through the light guide is merely the length of the light guide, $\mathrm{I}$, divided by $\mathrm{c} / \mathrm{n}$, where $\mathrm{c}$ is the speed of light and $\mathrm{n}$ is the index of refraction for the light guide material. This is known to be $1.2 \mathrm{~ns}$. This was 


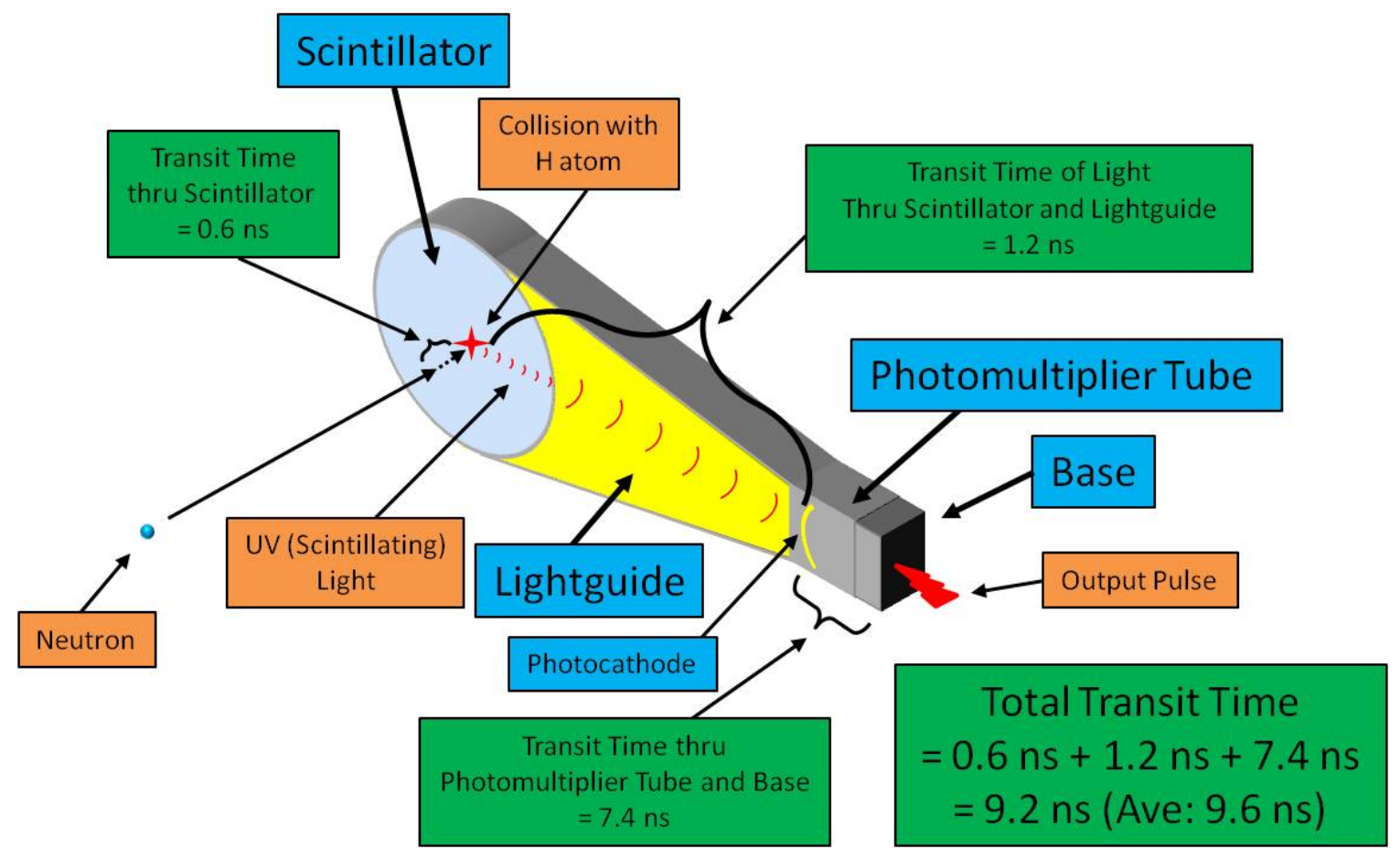

Figure 32. Schematic of the time delays that need to be taken into account (green boxes). The detector shown is a "single paddle" nTOF detector. A neutron (at left) penetrates the center of the scintillator and has a collision with a hydrogen atom. The time from the neutron entering the scintillator to when it interacts is its transit time, and was found above to be 0.6 ns. The time the UV (scintillating) light reaches the light guide (yellow), and travels the length of the light guide is $1.2 \mathrm{~ns}$. Upon reaching the photocathode in the photomultiplier tube, the light is converted into an electronic signal, emerging from the base with a transit time of 7.4 ns, measured by National Security Technologies (NSTec) [28]. The sum of all transit times are $9.2 \mathrm{~ns}$, but with fielding several nTOF detectors, it was considered that the average transit time to be $9.6 \mathrm{~ns}$.

taken from the center of the scintillator as an average value. Finally, the transit time through the photomultiplier tube and base, measured by NSTec [28], is 7.4 ns. The sum of all these delays is 9.2 ns. This was done for every nTOF detector fielded, with an average value to be $9.6 \mathrm{~ns}$ to be the total delay from when radiation impinges upon the face of the scintillator to the electronic signal coming out of the base of the photomultiplier tube.

Once all the time delays have been taken into account, it is then necessary to correct Figure 31 in terms where the actual time/neutron response starts. This is 
because when the time responses were found experimentally at Idaho State, there was no fiducial present to indicate the time radiation was impinging upon the detector.

Therefore, a value of $5 \%$ of the amplitude in Figure 31 was taken to be the start of the output pulse of the detector, and from that point, $9.6 \mathrm{~ns}$ earlier in time was taken to be the point at which radiation impinged upon the detector. This is shown in Figure 33.

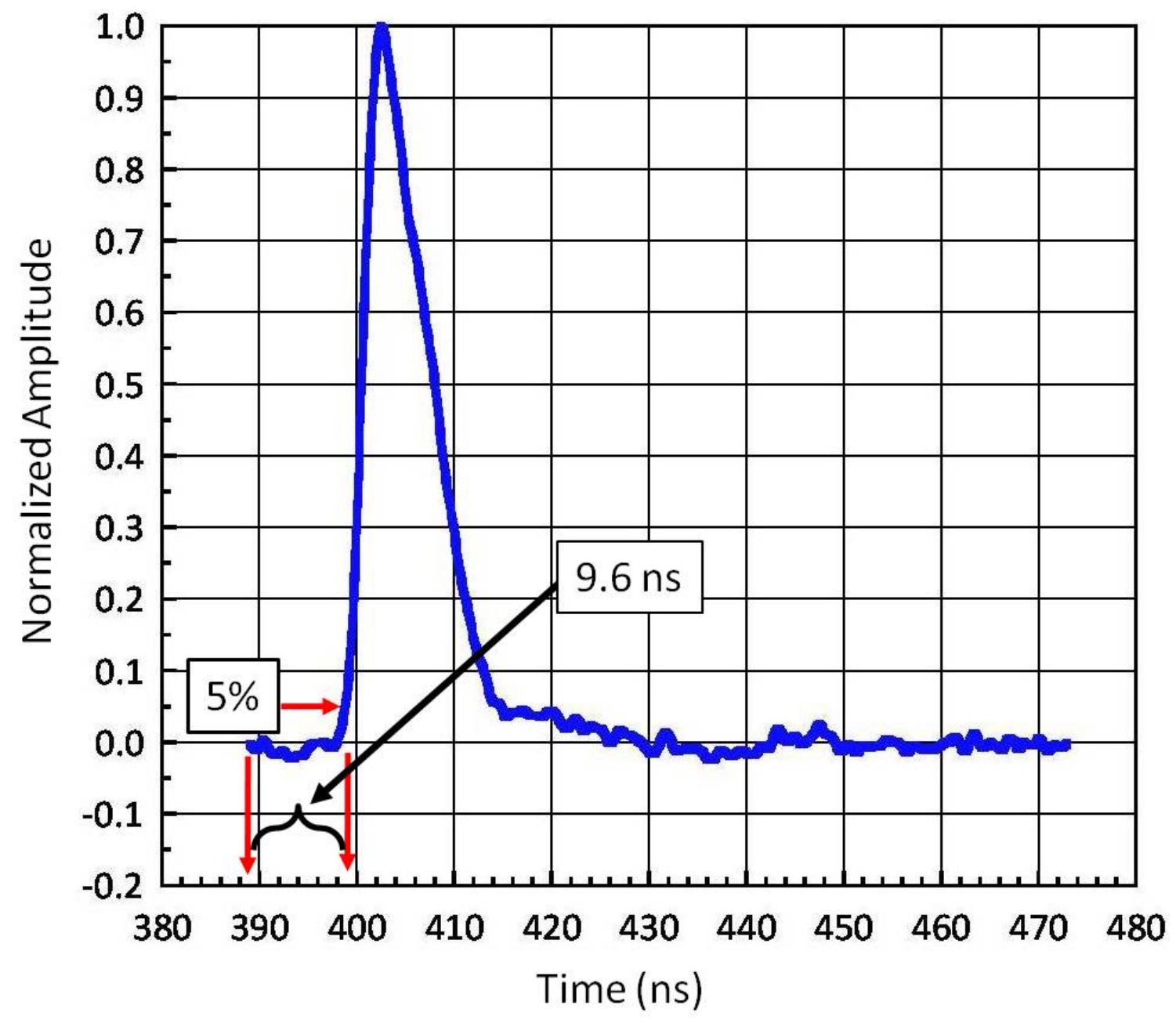

Figure 33. The Neutron Impulse/Time Response corrected for the time when radiation first impinges upon the detector. From the point of $5 \%$ of shot breakout, 9.6 ns earlier in time indicates the time in which radiation first impinges upon the detector (In this case, radiation first strikes the detector at $389.11 \mathrm{~ns}$ ). $9.6 \mathrm{~ns}$ is the time it takes for the signal to traverse the entire detector - scintillator, light guide, photomultiplier tube and base before an electronic signal (the pulse shown above) is produced. 


\section{CHAPTER 9}

DECONVOLVING THE NEUTRON AND TIMING INSTRUMENT RESPONSE OUT OF THE REAL DATA

The neutron and timing instrument response (the convolution of both timing and neutron impulse information) in Figure 33 was now placed in "wrap-around order" (see Figure 27) and deconvolved out of the empirical data. The result is shown in Figure 34. Note that the neutron peak is at $373.53 \mathrm{~ns}$ (see arrow), and that the neutron peak from the empirical data (Figure 26) is at 383.13 shakes. The deconvolution has shifted the waveform earlier in time, because the time response information has been removed from the data. The neutron peak has been shifted back in time by $9.6 \mathrm{~ns}$. This is a check that causality has not been violated. Neutrons must arrive earlier than the signal that is produced [29].

As a check to see if the deconvolution is correct, the waveform in Figure 34 was convolved with Figure 33 and compared with the empirical data. The results are shown in Figure 35 as an area normalized plot. The blue waveform is the empirical data from z1217, and the red waveform is the convolved signal. As can be seen, the red falls neatly on top of the blue with extremely small variations, indicating a very good fit.

\section{SUBTRACTING THE CONTRIBUTION DUE TO NEUTRON SCATTERING}

Once the time and neutron impulse response have been deconvolved out of the data, the contribution due to scattering can then be subtracted out. This is accomplished by running an "Ideal Case" case of a $2.54 \mathrm{~cm}$ (1 inch) scintillator $809 \mathrm{~cm}$ from a 4 keV DD fusion source (shown in Table II), with no material between the 


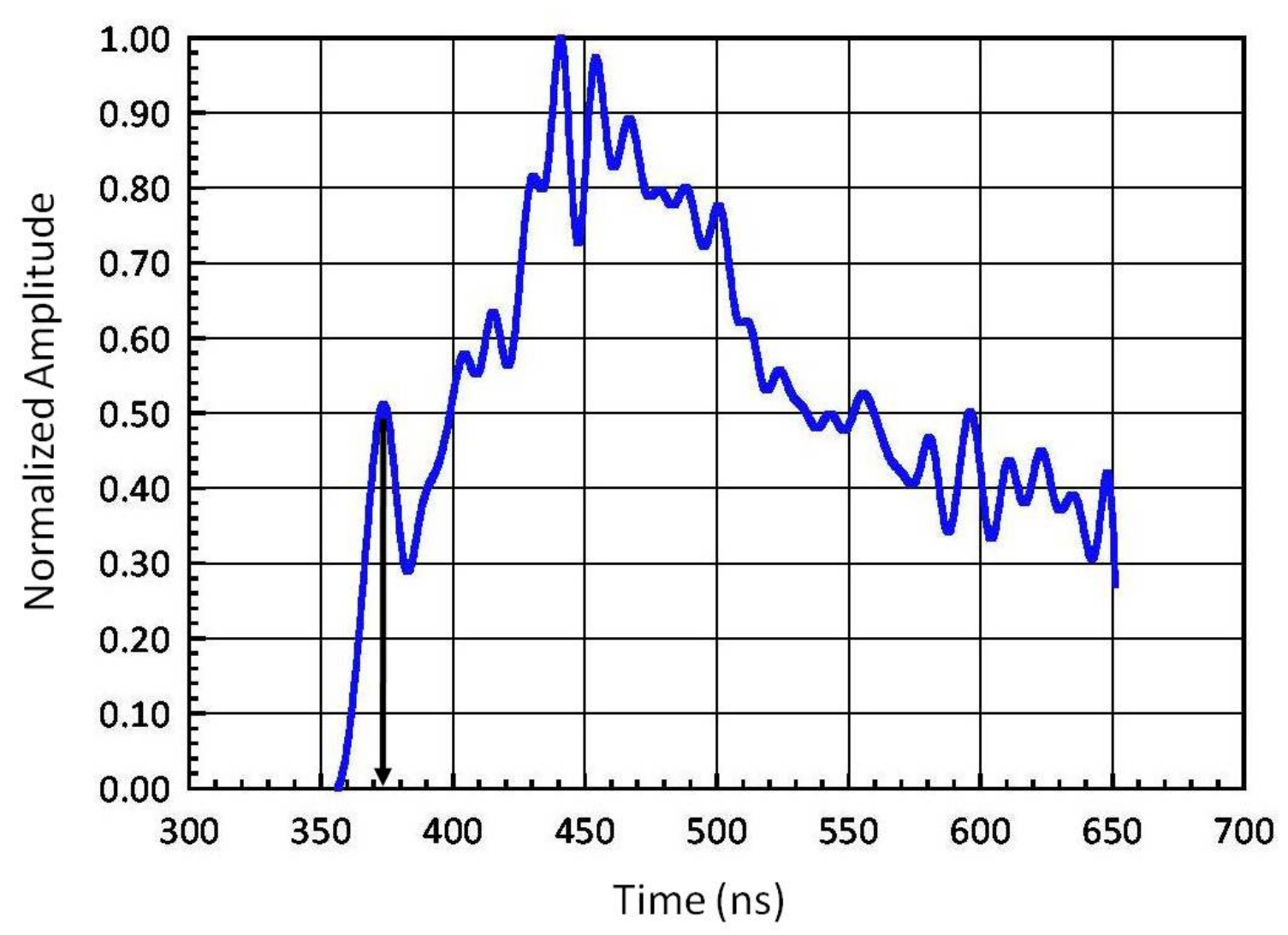

Figure 34. The empirical data from shot $z 1217$ with the neutron impulse and timing information (Figure 31) deconvolved out of it. Note that the neutron peak is at $373.53 \mathrm{~ns}$ (see arrow) vs the neutron peak in the empirical data (Figure 26) at $383.13 \mathrm{~ns}$. The deconvolution has shifted the waveform earlier in time by $9.6 \mathrm{~ns}$ - indicating that the time response information has been removed from the data. The delay above must be at least $9.6 \mathrm{~ns}$, in order that causality not be violated - neutrons must arrive earlier than the signal that is produced.

source and scintillator, and comparing a case run with all the geometry and materials in the problem with the $2.54 \mathrm{~cm}$ (1 inch) scintillator at the same location (shown in blue in Figure 15). A plot of the "Ideal Case" being subtracted out from the "Full Scale" geometry leaving the contribution to scattering is shown in Figure 36. It is interesting to note that there is a small hump (green) due to scattering early in time, indicating that some shallow angle scattering is occurring, contributing to the signal. Not much can be said for the tail, as this is dominated by the huge, second scattering peak produced from 
lack of a neutron collimator near the source. Looking at the full width at half maxima in Table III - subtracting the FWHM of the "Ideal Case" from the FWHM of the "Full Scale" geometry in Figure 36 - one sees that the broadening due to scattering is $3.857 \mathrm{~ns}$.

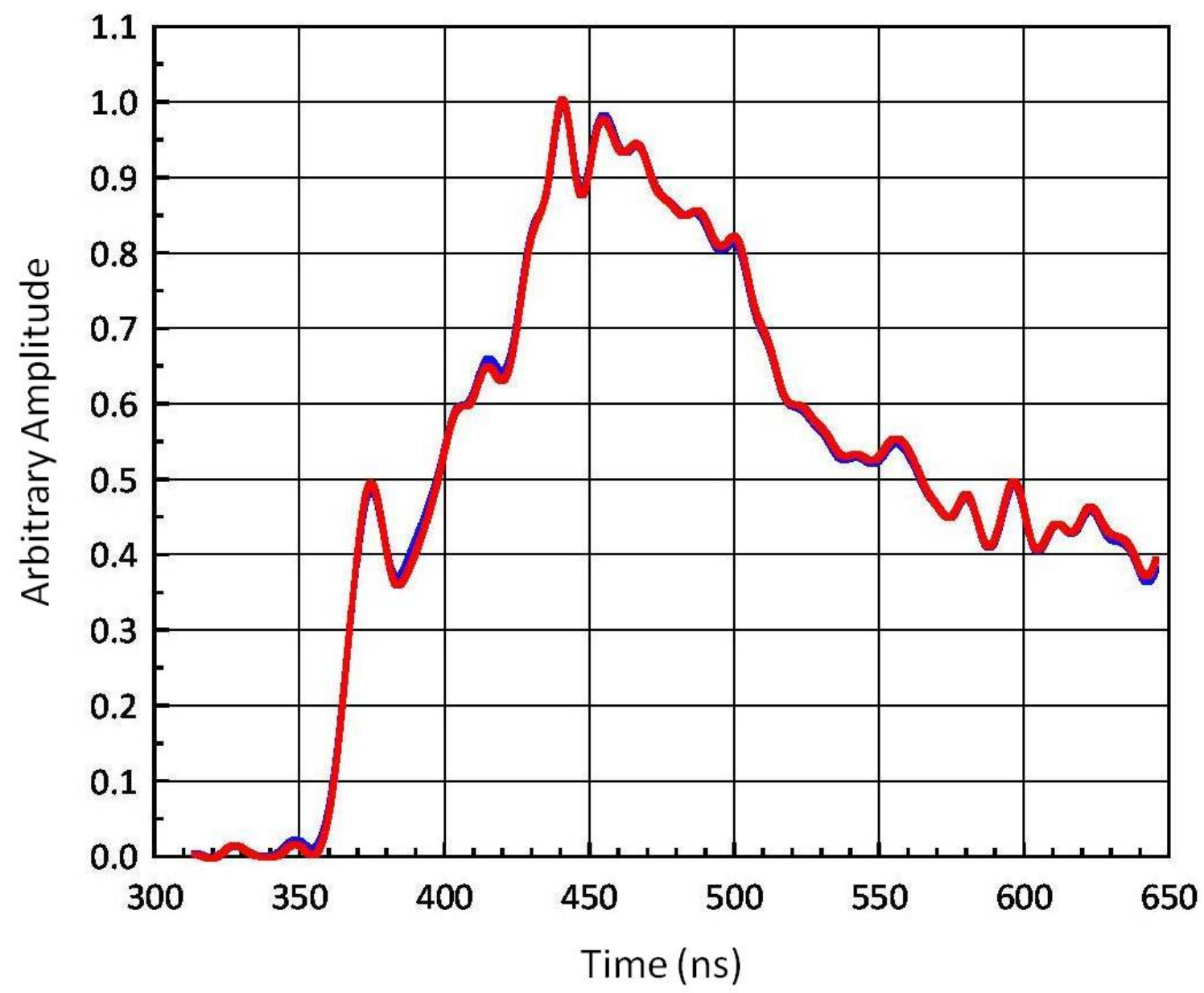

Figure 35. Area Normalized plot of the empirical data from z1217 (blue) compared with Figure 33 and Figure 34 being convolved together (red) as a "check" of the deconvolution. As can be seen, the red falls neatly on top of the blue with extremely small variations, indicating a very good fit. 


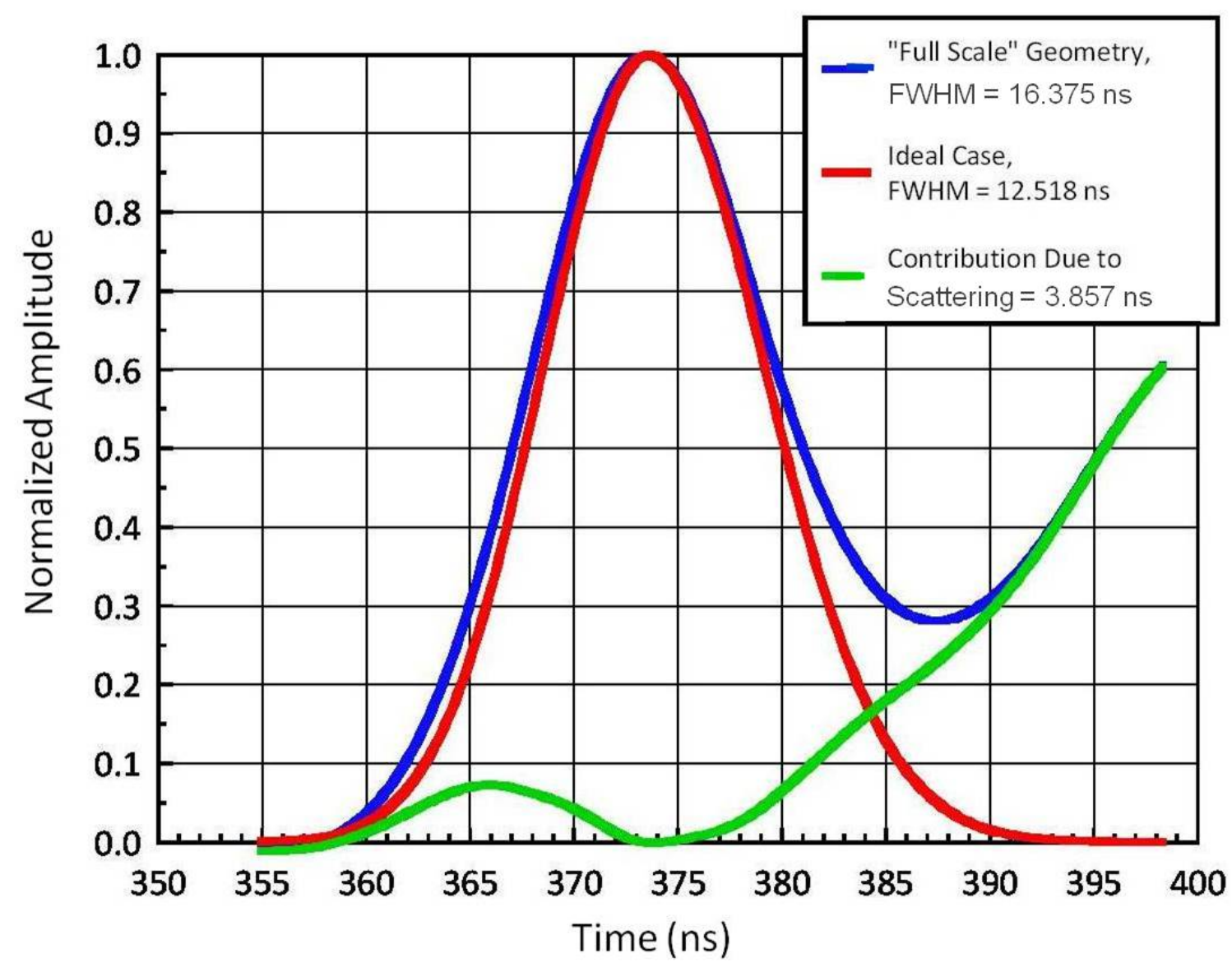

Figure 36. The "Ideal Case" (red) - i.e., a $2.54 \mathrm{~cm}$ (1 inch) scintillator placed $809 \mathrm{~cm}$ away from a 4 keV DD Fusion Source is subtracted out from the "Full Scale" Geometry (i.e., the scintillator in the same location but now with all the geometry of the problem added; blue). The result is the contribution due to scattering (green). Of interest is the small hump due to scattering early in time, indicating that indeed, there is some shallow-angle scattering contributing to the signal. Not much can be said for the tail, as this is dominated by the huge, second scattering peak produced from lack of a neutron collimator near the source. Subtracting the FWHM of the Ideal Case from the Full Scale Geometry leaves 3.857 ns, which is the amount of broadening that scattering contributes.

Once the contribution due to neutron scattering has been found (green in Figure 36), it could now be subtracted from the waveform in Figure 34 - the empirical data from z1217 with the time response and neutron impulse response deconvolved out of it. This is shown in Figure 37. The data is now ready to be transformed from the time domain $(d N / d t)$ to the energy domain $(d N / d E)$ to infer a neutron spectrum. 
Table III.

Broadening due to Scattering at Detector Location " $D$ " with no Collimator.

\begin{tabular}{|c|c|}
\hline Waveform & FWHM (ns) \\
\hline "Full Scale" Geometry (Bottom nTOF) & 16.375 \\
\hline "Ideal Case" & 12.518 \\
\hline Broadening Due to Scattering: & $16.375-12.518=\underline{3.857 \mathrm{~ns}}$ \\
\hline
\end{tabular}

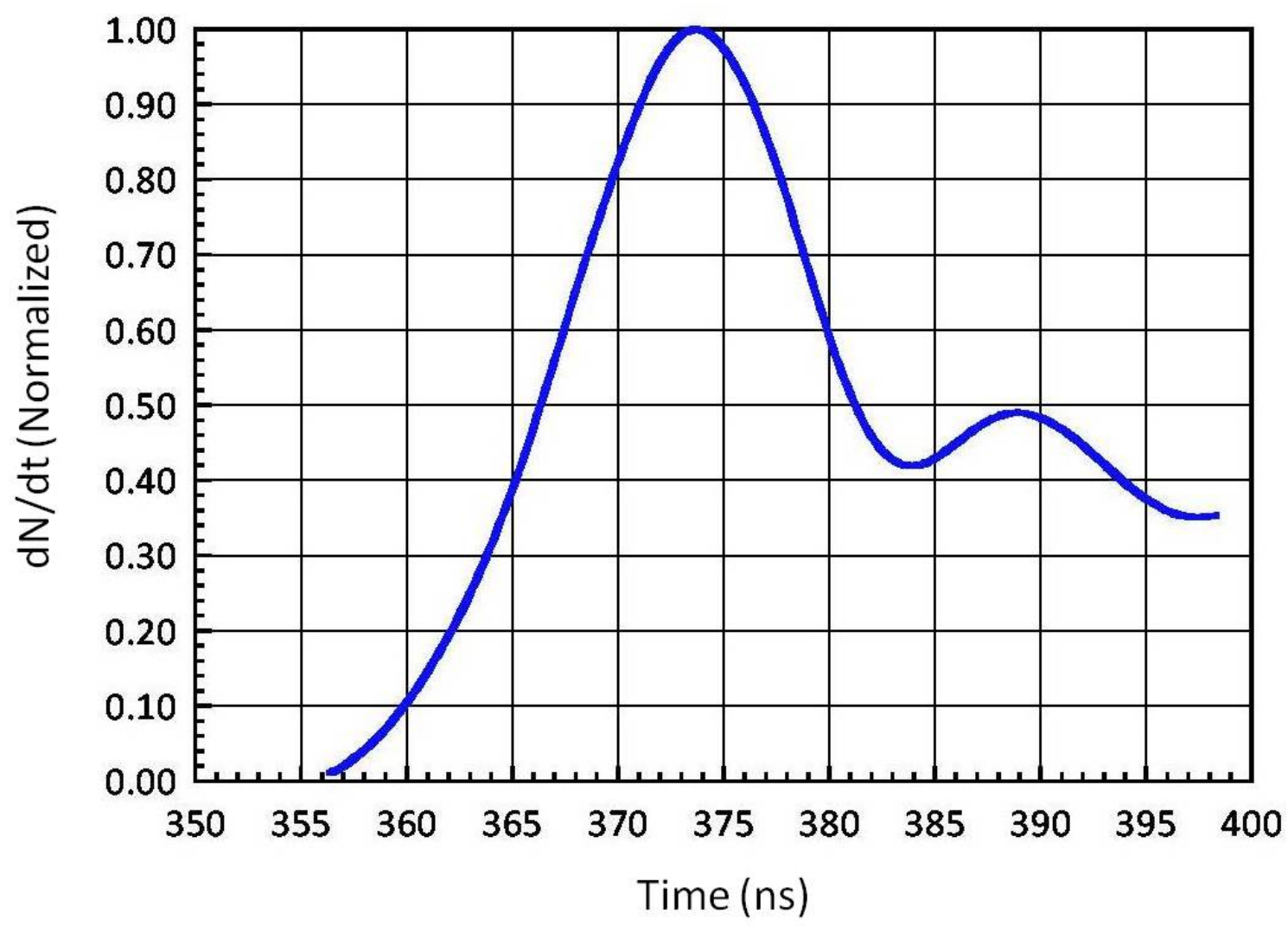

Figure 37. The empirical data from z1217 with the time response and neutron impulse response deconvolved out of it, and now with the contribution due to scattering (green in Figure 36) subtracted out of it. The data is now ready to be transformed from the time domain $(d N / d t)$ to the energy domain $(d N / d E)$ in order to produce a neutron spectrum. 


\section{CHAPTER 10}

\section{MAKING THE TRANSFORMATION FROM $(d N / d t)$ to $(d N / d E)$}

In the real world, the signal produced by an nTOF detector is in Voltage vs Time, due to the fact that the small amount of light output produced in the scintillator is converted to an electrical signal in the photomultiplier tube. The amplitude of this waveform is directly related to the number of incident neutrons that interacted in the scintillator. Thus, one could also refer to an nTOF signal as $d N / d t$, or number of neutrons interacting in the scintillator vs time. This is a known quantity. Unfortunately, what is not known is $d N / d E$, which is related to $d N / d t$ by:

$$
\frac{d N}{d t}=\frac{d N}{d E} * \frac{d E}{d t}
$$

Solving for $d N / d E$ :

$$
\frac{d N}{d E}=\frac{\frac{d N}{d t}}{\frac{d E}{d t}}
$$

From equation (9) above:

$$
E=\frac{1}{2} m v^{2}
$$

Putting $\mathrm{v}$ in terms of $\mathrm{t}$ :

$$
E=\frac{1}{2} m_{0} \frac{c^{2}}{c^{2}}\left(\frac{l}{t}\right)^{2}
$$

let $m=m_{0} c^{2}=939.5054 \mathrm{MeV}$, and taking the derivative of (22) with respect to $\mathrm{t}$ : 


$$
\frac{d E}{d t}=-\frac{m}{c^{2}} \frac{l^{2}}{t^{3}}
$$

Taking the absolute value of $d E / d t$ :

$$
\left|\frac{d E}{d t}\right|=+\frac{m}{c^{2}} \frac{l^{2}}{t^{3}}
$$

Solving for $t$ in equation (22):

$$
t=\sqrt{\frac{m l^{2}}{2 c^{2} E}}
$$

And $t^{3}$ becomes:

$$
t^{3}=\left[\frac{m l^{2}}{2 c^{2} E}\right]^{3 / 2}
$$

Substituting the value of $d E / d t$ in (24) into (20) becomes:

$$
\frac{d N}{d E}=\frac{d N}{d t} * \frac{c^{2} t^{3}}{m l^{2}}
$$

Substituting the value of $\mathrm{t}^{3}$ in (26) into (27):

$$
\frac{d N}{d E}=\frac{d N}{d t} * \frac{c^{2}}{m l^{2}}\left(\frac{m l^{2}}{2 c^{2} E}\right)^{3 / 2}
$$

Simplifying:

$$
\frac{d N}{d E}=\frac{m^{1 / 2} l}{2 c \sqrt{2}^{3 / 2}} * \frac{d N}{d t}
$$




\section{Defining constants:}

$$
\begin{gathered}
K(l)_{E}=\frac{m^{1 / 2} l}{2 c \sqrt{2}}, \text { and } K(l)_{t}=\frac{c^{2}}{m l^{2}} \text { such that: } \\
\frac{d N}{d E}=K(l)_{E} * \frac{d N}{d t} * E^{-3 / 2}
\end{gathered}
$$

and:

$$
\frac{d N}{d E}=K(l)_{t} * \frac{d N}{d t} * t^{3}
$$

Equation (31) is used to solve for $d N / d E$ and equation (22) to solve for $E$. The values of $d N / d t$ in (31) are those of the ordinate of Figure 37 and the values of $t$ are those of the abscissa of Figure 37. The units of equation (31) must be \#/MeV, and those of equation (22) are MeV. A plot of the transformation from $d N / d t$ from Figure 37 to $d N / d E$ is shown in Figure 38. It is the neutron spectrum for shot z1217 at detector location " $D$ " in Figure 1. The time response (Figure 13), and the neutron impulse response (Figure 29), convolved together (Figure 31) to include both timing information and neutron impulse information (Figure 31) was deconvolved out of the data (Figure 34), and the contribution to scattering was subtracted out (Figure 36, green), leaving the true $d N / d t$ signal (Figure 37). The transformation to $d N / d E$ - the neutron spectrum is shown in Figure 38. It should be noted that the energy bins along the ordinate are not equal after the transformation is made, with larger bins at high energies, but the data can be interpolated with the bin width of the smallest energy bin at the extreme end of the data. 


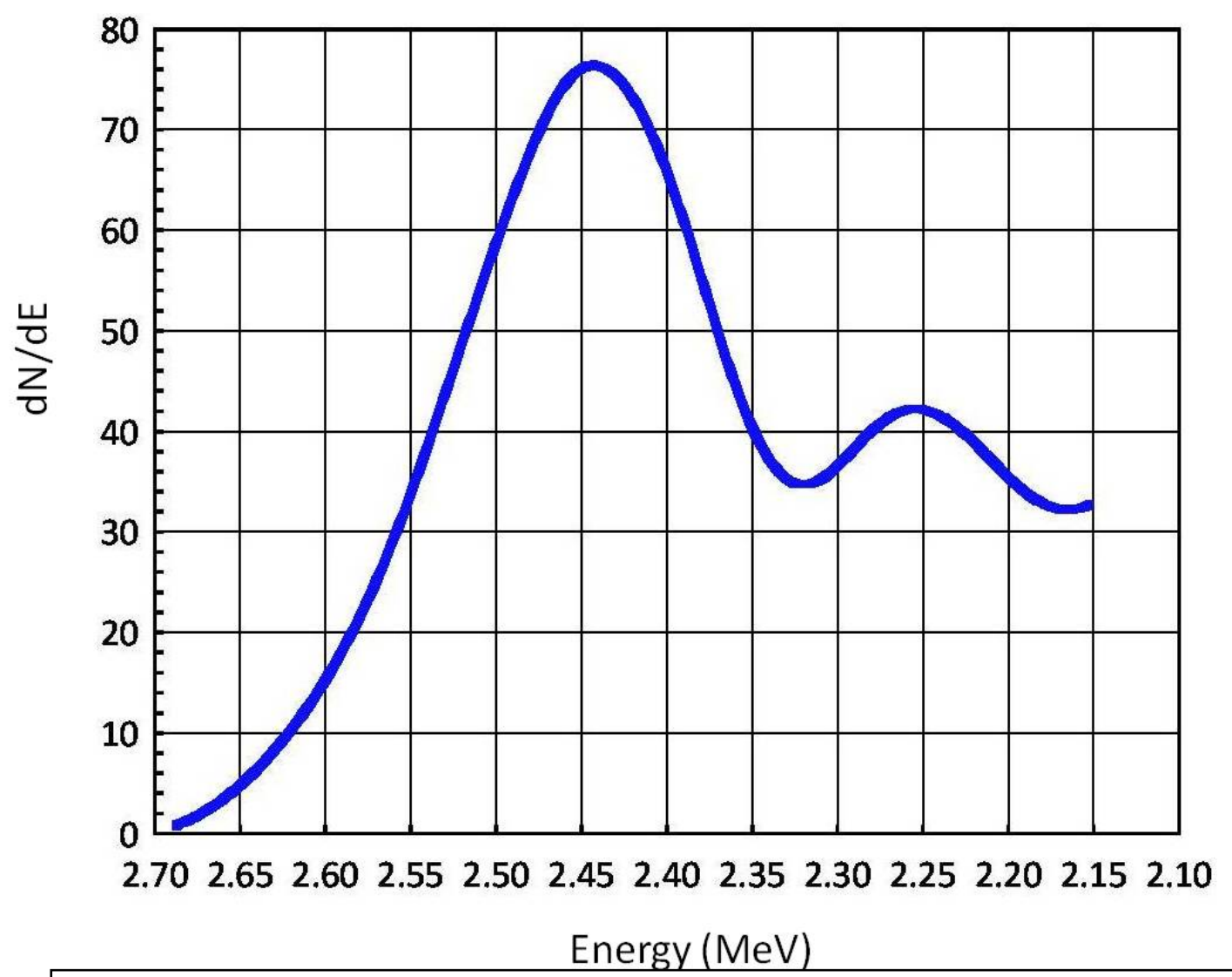

Figure 38. The Transformation from $d N / d t$ (Figure 34) to $d N / d E$, the neutron spectrum for shot z1217 at detector location " $D$ " in Figure 1. The time response and neutron impulse response have been deconvolved out of the data, and the contribution due to scattering has been subtracted out. The humped "tail" seen at $\sim 2.25 \mathrm{MeV}$ is not to be believed because of the enormous scattering tail (green in Figure 36 ).

According to the equation:

$$
\int_{E_{\min }}^{E^{\max }} \frac{d N}{d E} d E=\int_{t_{\min }}^{t^{\max }} \frac{d N}{d t} d t
$$

the integral of $d N / d E$ in Figure 38 must equal the integral of $d N / d t$ in Figure 37. Before integrating $d N / \mathrm{dE}$ (Figure 38), the smallest bin width found at the end of the data must be used to interpolate the data, because all the bin widths need to be the same before integrating. Table IV below shows that both integrals are the same. 
Table IV.

Integrals of $d N / d t$ (Figure 37 ) and $d N / d E$ (Figure 38)

\begin{tabular}{|c|c|}
\hline Waveform & $\underline{\text { Integral }}$ \\
\hline$d N / d t$ (Figure 37) & 1.84385 \\
\hline$d N / d E$ (Figure 38) & 1.84386 \\
\hline
\end{tabular}

For the nTOF detector located at position " $\mathrm{C}$ " in Figure 1, the top nTOF in the basement "pig", the same analysis was performed, and the contribution due to scattering is shown in Figure 39 (green). It was found by subtracting the "Ideal Case" full width at half maxima from the "Full Scale" geometry full width at half maxima. The broadening due to scattering at detector location " $C$ " in Figure 1 with no collimator is 3.89 ns.

The contribution to scattering in the top nTOF was subtracted from the data after having the time response and neutron impulse response deconvolved out of it, and the transformation from $d N / d t$ to $d N / d E$ was made. It is shown in Figure 40 , plotted alongside the spectrum found at the bottom nTOF location (Figure 38). The Bottom nTOF spectrum's peak is located at $2.46 \mathrm{MeV}$, and the top nTOF spectrum's peak is at 2.44 MeV. The "tails" are not to be believed, since scattering was such an issue. Later on in Z's history the Ultra-High Molecular Weight (UHMW) TIVAR collimator (Figure 19) was added to neutron producing shots to "clean up" the neutron signals. 


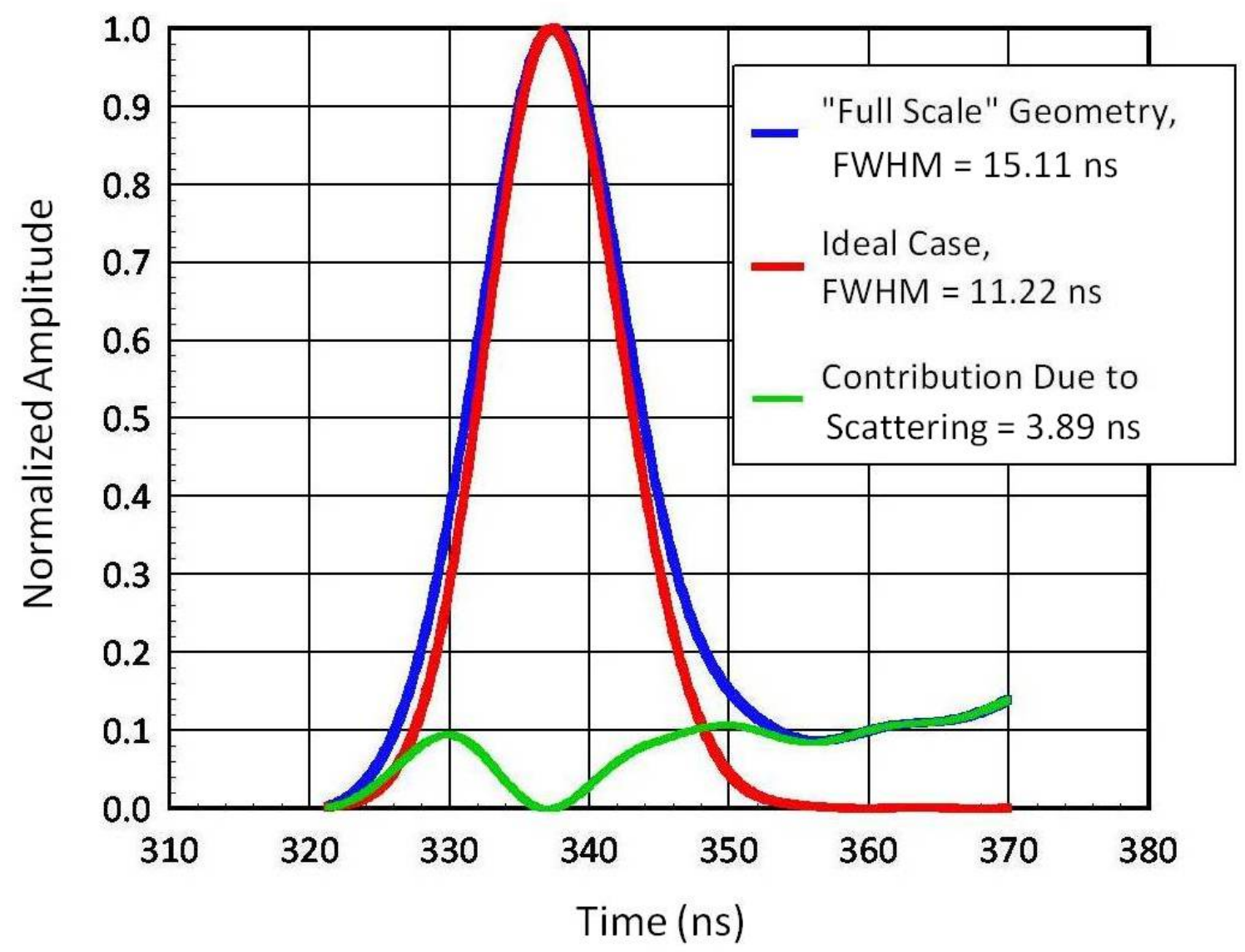

Figure 39. The "Ideal Case" (red) - i.e., a $2.54 \mathrm{~cm}$ (1 inch) scintillator placed $730 \mathrm{~cm}$ (location " $C$ " in Figure 1) away from a $4 \mathrm{keV}$ DD Fusion Source is subtracted out from the "Full Scale" Geometry (blue). The result is the contribution due to scattering (green). Subtracting the FWHM of the "Ideal Case" from the FWHM of the "Full Scale" Geometry leaves $3.89 \mathrm{~ns}$, which is the amount of broadening that scattering contributes.

And as shown in Figures 22 and 24, it greatly reduced the second scattering "tail" for detector location " $\mathrm{D}$ " (Figure 1) and virtually eliminated the second scattering tail at detector location "C" (Figure 1).

On shot z1549 both detector signals from the basement "pig" were analyzed. Once the time response and neutron impulse response was deconvolved out of the data, the contribution to scattering was determined for detector location " $D$ " (Figure 1) and shown in Figure 41. The "Full Scale" geometry (blue) was run with the TIVAR 1000 
collimator in place in the model, and it is compared to an "Ideal Case" (red) in Figure 41. The broadening due to scattering (green) is the "Ideal Case" subtracted from the "Full Scale" geometry, and is 2.849 ns.

Table V.

Broadening due to Scattering at Detector Location " $C$ " with no Collimator.

\begin{tabular}{|c|c|}
\hline Waveform & FWHM (ns) \\
\hline "Full Scale" Geometry (Bottom nTOF) & 15.11 \\
\hline "Ideal Case" & 11.220 \\
\hline Broadening Due to Scattering: & $15.11-11.220=\underline{3.89 \mathrm{~ns}}$ \\
\hline
\end{tabular}

This is less than the value of 3.857 ns shown in Figure 36 and Table III, indicating that the collimator is reducing some shallow-angle scattering into the detector. Some shallow-angle scattering is still contributing to the signal - this is due to the fact that the "bore" on the collimator is quite large, $7.62 \mathrm{~cm}$ ( 3 inch) diameter, and as shown in Figure 20, the "collimator cone" spreads out to a $116 \mathrm{~cm}$ (45.7 in) diameter at the basement floor. The collimator also reduces the second scattering tail drastically in Figure 41 compared to Figure 36.

Analysis of the top nTOF signal when the collimator was added (position " $\mathrm{C}$ " in Figure 1) was performed and is shown in Figure 42. The "Full Scale" geometry (blue) was run with the TIVAR 1000 collimator in place, and it is compared to the "Ideal Case" 


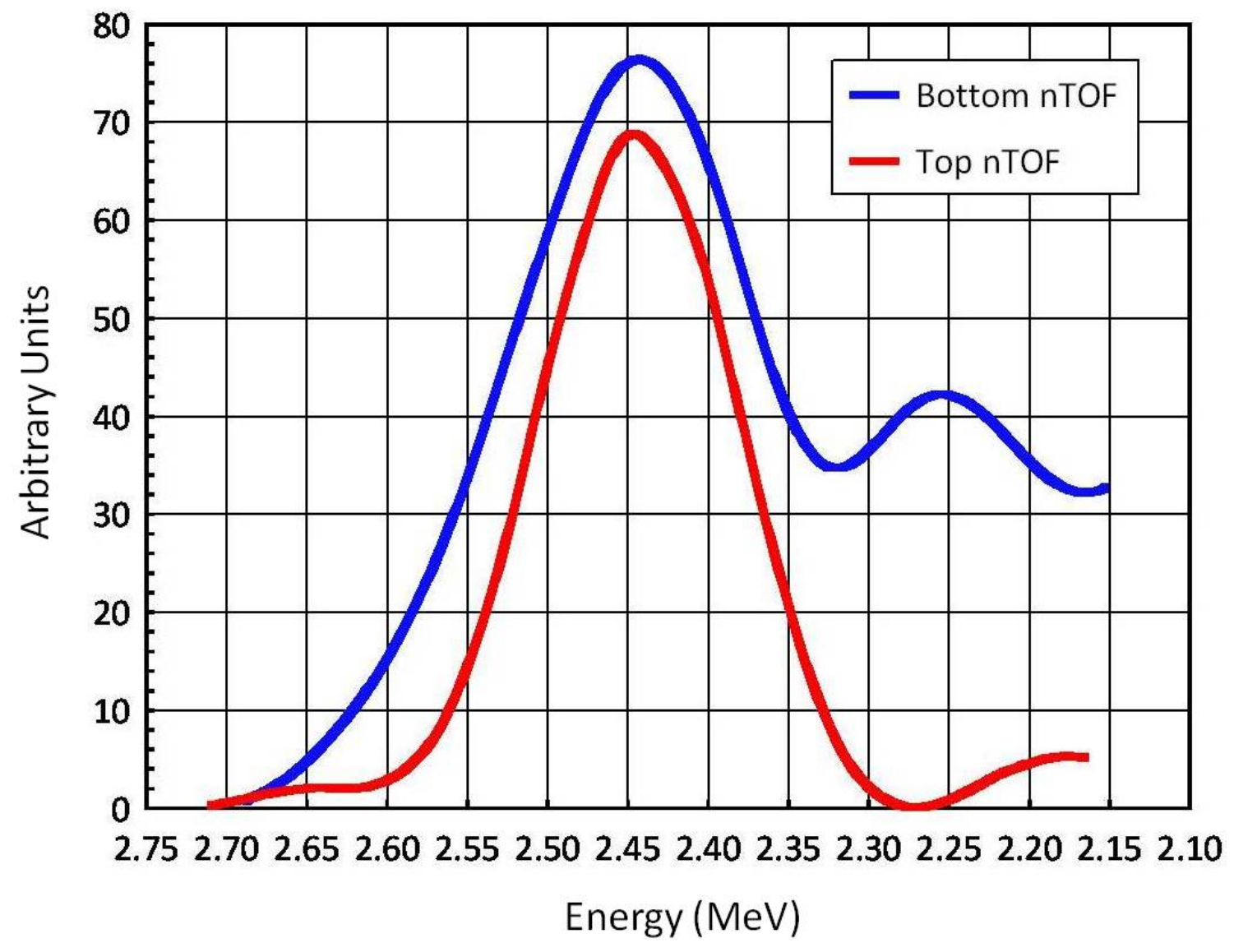

Figure 40. Energy Spectra for both the Top and Bottom nTOF detectors at locations " $C$ " and " $D$ " in Figure 1 from shot z1217. Although not identical, they are similar in shape. The Bottom nTOF spectrum's peak is at $2.46 \mathrm{MeV}$, while the Top nTOF spectrum's peak is at $2.44 \mathrm{MeV}$. Note that Energy along the abscissa decreases as one moves to the right. The "tails" are not to be believed, since scattering was such an issue.

(red). The broadening to scattering (green) is the "Ideal Case" subtracted from the "Full Scale" geometry, and is $3.176 \mathrm{~ns}$, shown in Table VII. This is less than 3.89 ns shown in Figure 39 and Table V, indicating that again, the collimator is reducing shallow angle scattering into the detector. And, as in the case of Figure 41, the collimator does effectively reduce the second scattering tail seen in Figure 39. 
Table VIII shows the contributions due to scattering for both detectors, with and

without the collimator. The addition of the collimator reduced the broadening due to scattering for detector location " $D$ " in Figure 1 (the Bottom nTOF) by $26.1 \%$. The

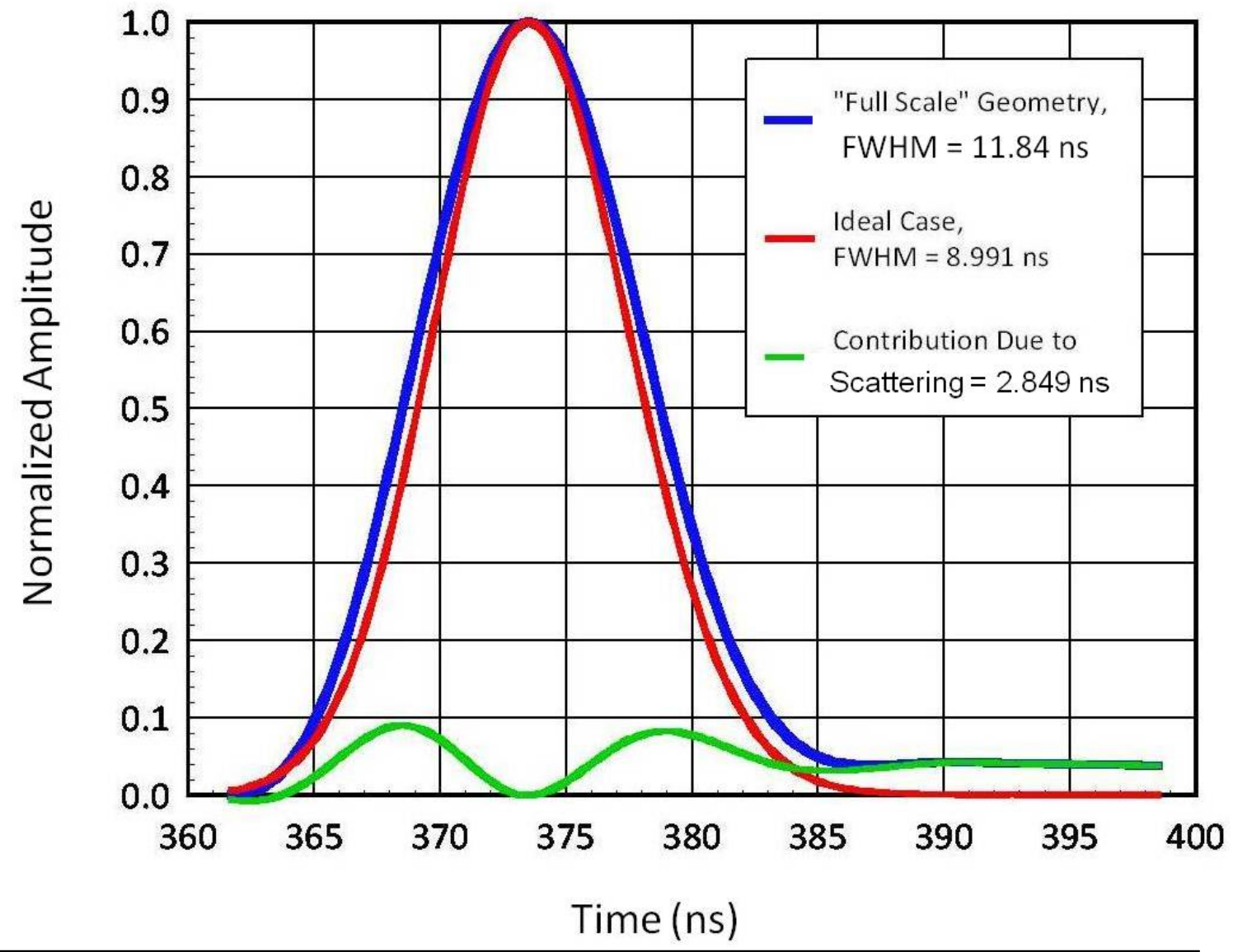

Figure 41. The "Ideal Case" (red) - i.e., a $2.54 \mathrm{~cm}$ (1 inch) scintillator placed $809 \mathrm{~cm}$ away from a 2 keV DD Fusion Source is subtracted out from the "Full Scale" Geometry (including the TIVAR collimator; blue). The result is the contribution due to scattering (green). What clearly can be seen is the reduction of the scattering tail in Figure 38 compared to Figure 33 - this is a direct result of the addition of the Tivar collimator. Subtracting the FWHM of the "Ideal Case" from the FWHM of the "Full Scale" Geometry leaves 2.849 ns, which is the amount of broadening that scattering contributes at detector location " $D$ " in Figure 1.

addition of the collimator reduced the broadening due to scattering for detector location " $\mathrm{C}$ " in Figure 1 (the Top nTOF) by $18.4 \%$. Reduction of shallow angle scattering could be increased even further if the "bore" of the collimator were reduced in size 
from $7.62 \mathrm{~cm}$ ( 3 inches) to a smaller diameter, but as shown in Figure 21, reducing the diameter would occlude the scintillators' view of the source, because the "pig" is tilted $3^{\circ}$ from vertical.

Table VI.

Broadening due to Scattering at Detector Location “ $D$ ” with a Collimator.

\begin{tabular}{|c|c|}
\hline Waveform & FWHM (ns) \\
\hline “Full Scale" Geometry (Bottom nTOF) & 11.84 \\
\hline "Ideal Case" & 8.991 \\
\hline Broadening due to Scattering: & $11.84-8.991=\underline{2.849 \mathrm{~ns}}$ \\
\hline
\end{tabular}

The contribution to scattering in both the Bottom nTOF and the Top nTOF was subtracted from the data after having the time response and neutron impulse response deconvolved out of them, and the transformation from $d N / d t$ to $d N / d E$ was made. Both spectra are shown in Figure 43. The Bottom nTOF spectrum's peak is at $2.44 \mathrm{MeV}$, while the Top nTOF spectrum's peak is located at $2.45 \mathrm{MeV}$. 


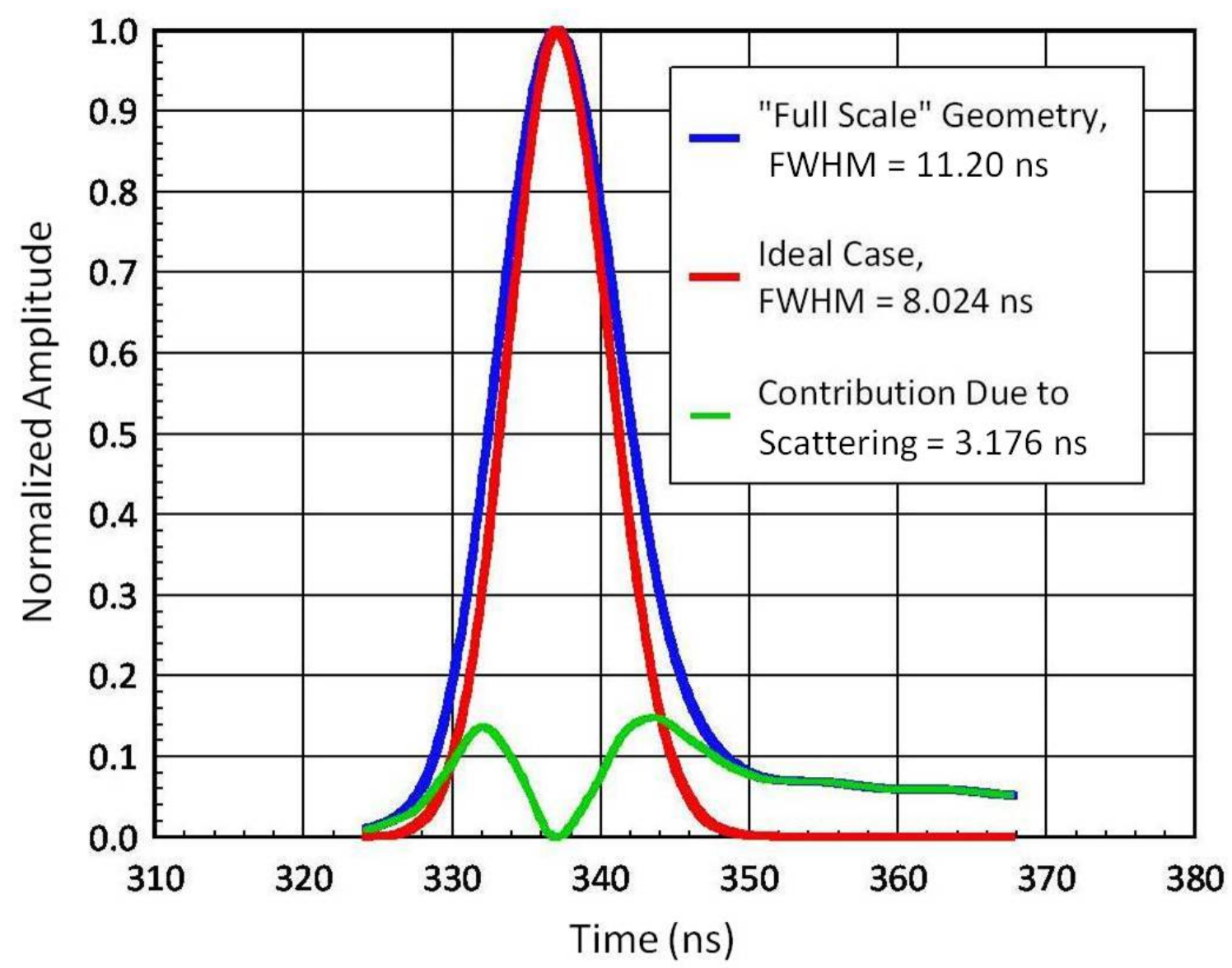

Figure 42. The "Ideal Case" (red) is subtracted from the "Full Scale" geometry (blue) to produce the contribution to scattering (green). This is the top nTOF located at position " $\mathrm{C}$ " in Figure 1, and was modeled at a temperature of $2 \mathrm{keV}$. The collimator does effectively reduce the second scattering peak shown in Figure 22. Subtracting the FWHM of the "Ideal Case" from the "Full Scale" Geometry leaves 3.176 ns, which is the amount of broadening that scattering contributes. 
Table VII.

Broadening due to Scattering at Detector Location " $\mathrm{C}$ " with a Collimator

\begin{tabular}{|c|c|}
\hline Waveform & FWHM (ns) \\
\hline “Full Scale" Geometry (Top nTOF) & 11.20 \\
\hline "Ideal Case" & 8.024 \\
\hline Broadening due to Scattering: & $11.2-8.024=\underline{3.176 \mathrm{~ns}}$ \\
\hline
\end{tabular}

Table VIII.

Broadening Due to Scattering for each Detector Location

\begin{tabular}{|c|c|c|c|}
\hline & Without Collimator & With Collimator (ns) & \% Reduction \\
\hline Bottom nTOF, (“D”) & 3.857 & 2.849 & 26.1 \\
\hline Top nTOF, ("C") & 3.89 & 3.176 & 18.4 \\
\hline
\end{tabular}




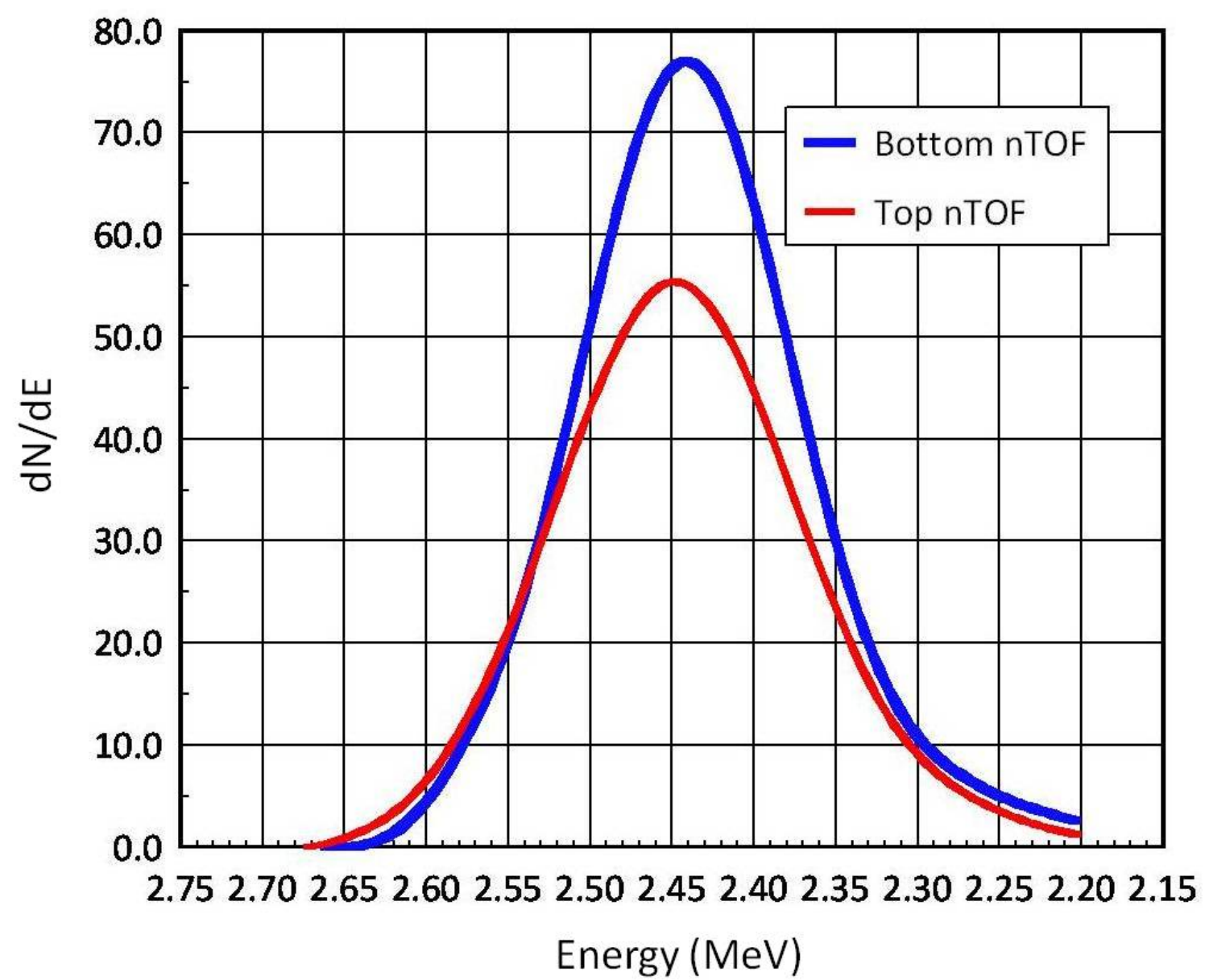

Figure 43. Energy Spectra for both the Top and Bottom nTOF detectors at locations " $\mathrm{C}$ " and " $D$ " in Figure 1 from shot z1549. They are similar in shape. The Bottom nTOF spectrum's peak is located at $2.44 \mathrm{MeV}$, and the Top nTOF spectrum's peak is at $2.45 \mathrm{MeV}$. Note that Energy along the abscissa decreases as one moves to the right. 


\section{CHAPTER 11}

\section{IDENTIFYING SOURCES OF NEUTRON SCATTERING}

An ideal neutron measurement would consist of detecting only those neutrons born at the source which arrive at the detector without interacting with any structural material in between. Experimentally, this can be difficult if not impossible, and depends on the facility, and the detector location. Collimation between the source and detector can greatly improve neutron signals, but may or may not be viable, depending on the facility. Therefore neutrons born at the source can and do undergo scattering off structural material and arrive at the detector, thereby "clouding" the pure signal, and making analysis of the plasma conditions at the source more difficult. One of the versatile aspects of this process described herein, however, is that the user -- upon suspecting certain materials to be contributors to neutron scattering - can actually test if they are indeed a cause of concern. By changing cells' importances to zero in the input deck (so that neutrons are killed when entering the cell) and changing the material that occupies the cells to a void, the user can then run the code, plot the light output and examine the detector response. The user can also identify whether photons or neutrons are responsible for any changes seen in the output, because the postprocessing code can be easily modified to look at just the contribution of light output made from neutrons, photons, or both. This is illustrated in Figure 44 . While the "mode" card in the input deck was turned on for both neutrons and photons, the source was a DD fusion neutron source and photons could only be produced by $n$,gamma capture reactions, therefore, virtually all the signal was produced from neutrons. As can 
be seen, following the primary neutron signal, there are two "humps" caused by neutrons scattering into the detector later in time. The first "hump" is caused by neutrons scattering in through the sides of the pig, and the second "hump" is from neutrons scattering off the elevator floor.

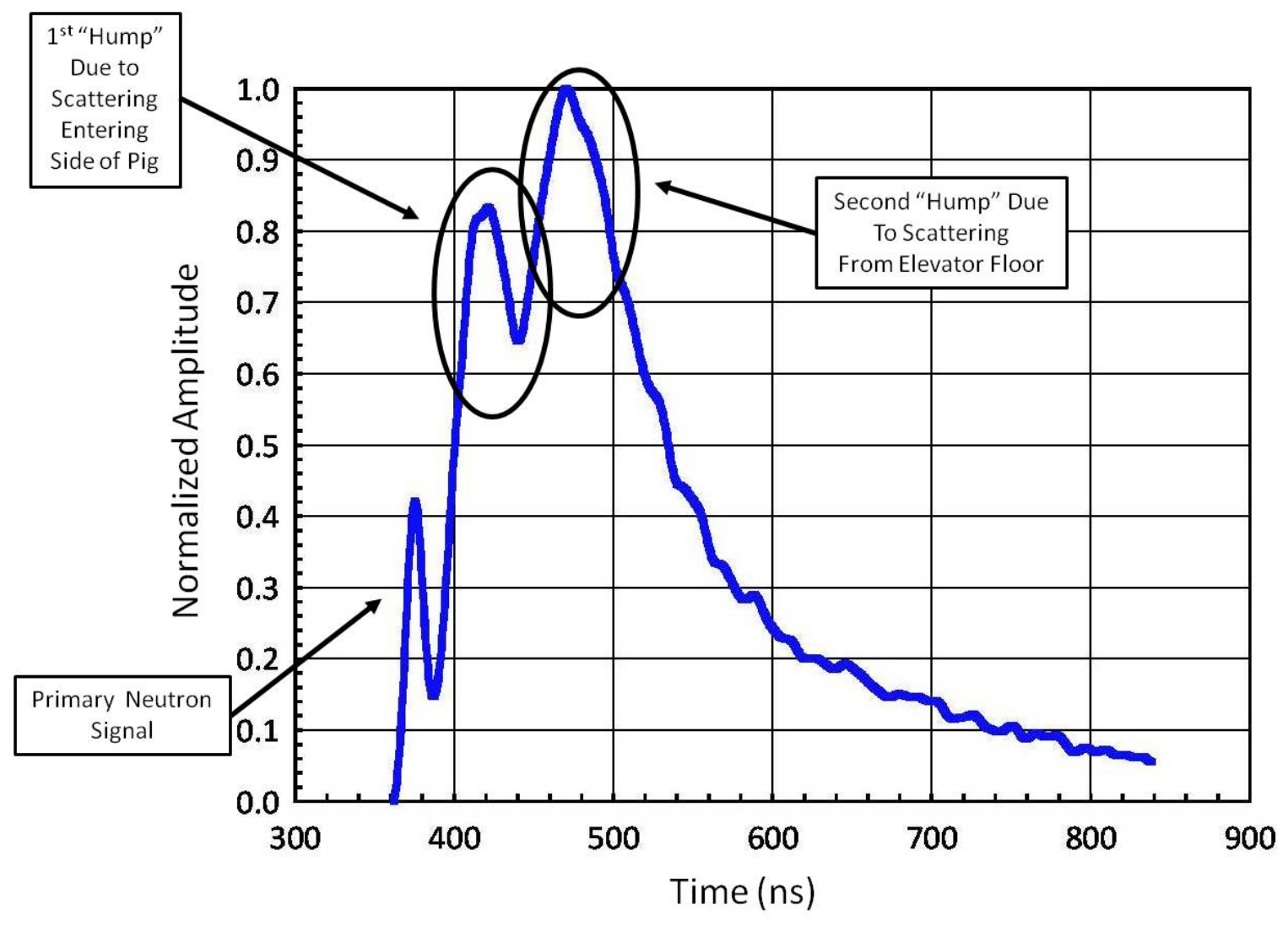

Figure 44. Neutron scattering after the primary pulse for detector location " $D$ " in Figure 1. As can be seen, the first "hump" is from neutrons scattering through the side of the pig, and the second "hump" is caused from neutrons scattering off the elevator floor and arriving at the detector later in time.

The input deck was then modified by changing all the neutron importances for all the cells comprising the elevator floor to zero, and changing the cells of the elevator from steel to a void. This causes all neutrons that interact with the elevator to be "killed" (i.e., removed from the problem). The result is shown in Figure 45. Note the 
second "hump" which was at $470 \mathrm{~ns}$ is now gone, confirming that it is indeed due to neutrons scattering off the elevator floor. The first "hump" is still there, indicating that neutrons are still scattering in through the sides of the pig.

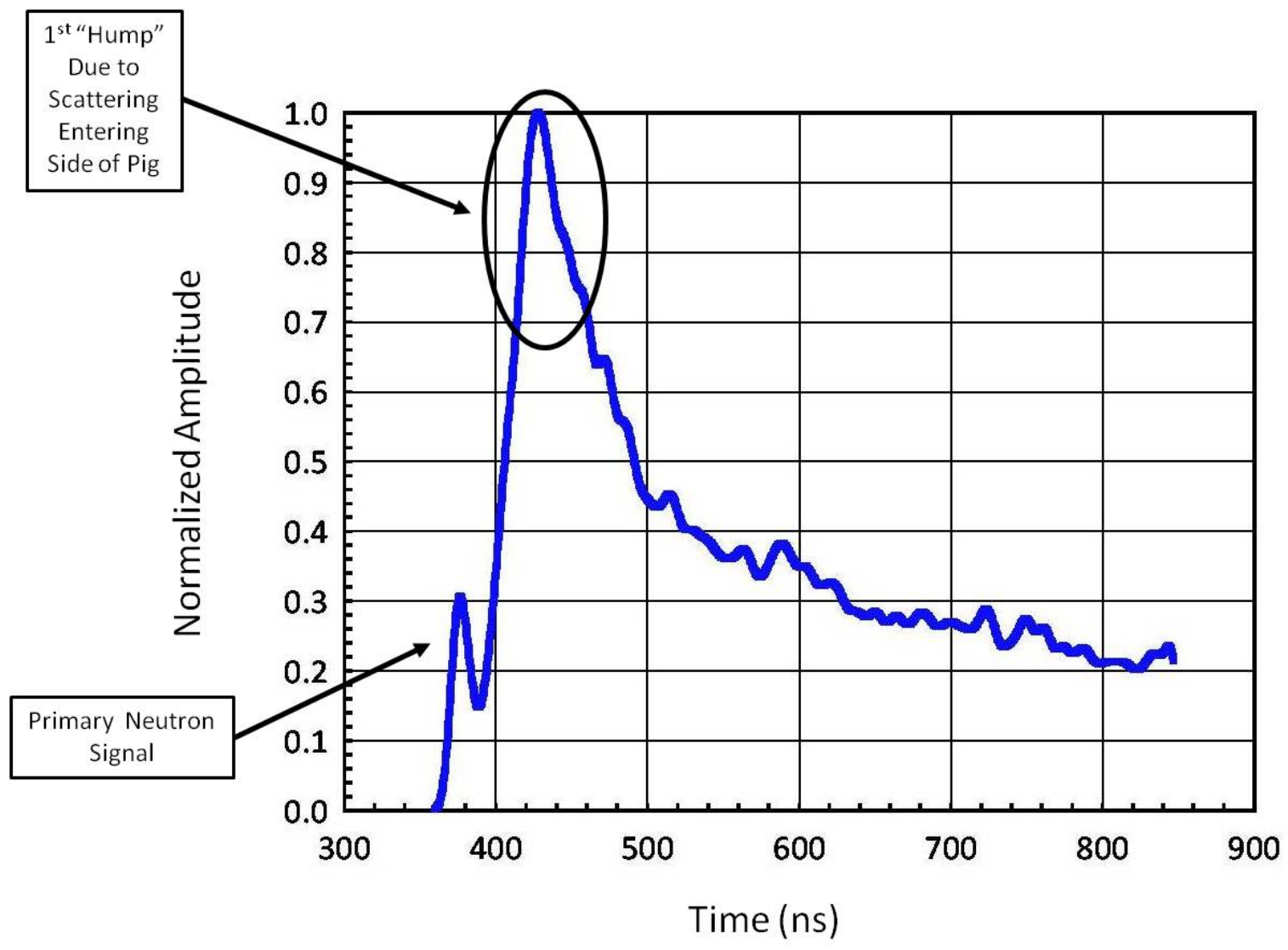

Figure 45. The elevator was made into a "kill" zone - i.e., any neutrons interacting with it were removed from the problem. The second "hump" seen in Figure 44 at $\sim 470$ ns is now gone, indicating that the elevator was indeed a cause of the second "hump". The first "hump" is still there, indicating that neutrons are still scattering in through the sides of the pig.

The input deck was again modified by changing all the cell importances that comprised the sides of the pig to zero and changing their material from steel to a void (this is in addition to the elevator as shown in Figure 45). The result is shown in Figure 46. Note the first "hump" which appeared at $\sim 430 \mathrm{~ns}$ is now gone, indicating that 
neutrons were indeed scattering in through the sides of the pig. All that is left is the primary neutron signal.

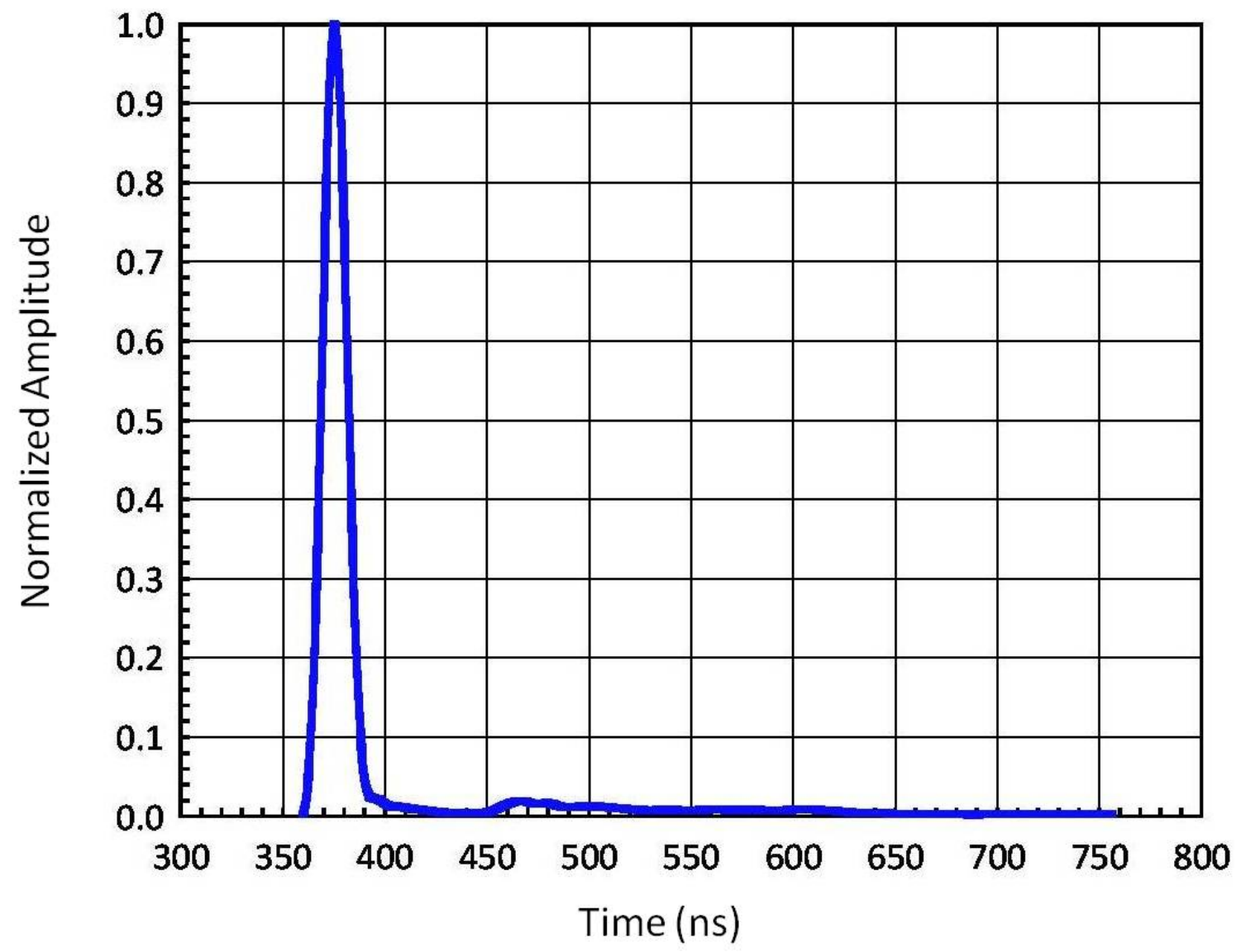

Figure 46. For the nTOF detector located at position " $D$ " in Figure 1, both the elevator and the sides of the pig have been made into "kill" zones, thereby removing neutrons that interact with them, indicating that both the elevator and the sides of the pig were the contributors to scattering peaks later in time. All that is left above is the primary neutron peak.

In this way, this technique can be very useful in identifying sources of neutron scattering, and mitigating them if possible (by removing hardware that is a source of scattering near a detector, for example) or by adding neutron shielding in key areas identified by the code. 


\section{CHAPTER 12}

\section{CONCLUSIONS}

A novel method of modeling the neutron time of flight (nTOF) detector response in current mode to inertial confinement fusion experiments has been presented. This process was first developed and then applied to the axial neutron time of flight detectors at the Z-Facility. First, the Z-Facility was modeled between the source and detector locations, which encompassed over 2400 cells and 900 surfaces with a usermodified version of MCNP, namely MCPN-PoliMi, which was developed by Enrico Padovani and Sara Pozzi in 2002. In order to obtain good statistics, many variance reduction techniques were utilized. MCNP-PoliMi simulates the detection of neutrons and photons in a plastic scintillator, and produces a collision data output table containing information of the incident particle (neutron or photon), target nucleus (hydrogen or carbon), energy deposited (MeV) and the time at which it occurred (shakes, [30]). A post-processing code was written to read this collision data output table. This converted the energy deposited by neutron and photon interactions in the plastic scintillator (i.e., nTOF detector) into light output, in units of MeVee (electron equivalent) vs time. A monoenergetic neutron case of $2.45 \mathrm{MeV}$ was run at each detector location and convolved with the experimental time response found at the Idaho Accelerator Center (IAC) using their $15 \mathrm{MeV}$ short pulse Linac with a 50 ps pulse width. This was done to provide both timing and neutron impulse response information. The resulting waveform was convolved with the simulated data and compared with the empirical results at each detector location, and was shown to be in good agreement. 
For each detector, an experiment was performed, first without a neutron collimator below the source, and then with a neutron collimator fielded below the source. It was shown that the addition of the collimator resulted in greatly reducing the second scattering peak in both detector signals, but also reduced shallow-angle scattering in the bottom nTOF by $26.1 \%$, and $18.4 \%$ in the top nTOF.

Then, as an additional step, the time response was deconvolved out of the empirical data. The contribution due to scattering was found by running a "Ideal Case" (i.e., nothing between the source and scintillator at each detector location); then a "Full Scale" geometry was run with all the structure added, and then subtracting one from the other. This scattering contribution was then subtracted from the empirical data. The resulting waveform was transformed from $d N / d t$ to $d N / d E$, in order to produce neutron spectra for each detector location (Top and Bottom nTOF) and for each configuration (without collimator and with collimator).

The method developed here can be used to simulate the detector response of any nTOF detector, with any digitized resolution, at any facility. It has been found useful to address key issues such as scattering, which always plays a role in neutron detection when using nTOF detectors. It can be used to identify sources of scattering as well, and to improve neutron signals by modeling effective collimation. It is hoped by the author that this method will prove to be a useful tool in future modeling of experiments, where "clean" neutron signals will provide the greatest amount of information from whence they came. 


\section{FUTURE WORK}

The techniques described herein have been shown to be extremely valuable in analyzing the data from dynamic holhraum experiments on Sandia National Laboratories' Z-machine. These techniques allowed the true neutron pulse shapes in the bottom nTOF detectors to be deconvolved from measured signals which in turn allows the determination of the neutron spectrum, the plasma ion temperature and the neutron yield. Since z-pinch fusion plasmas have historically been dominated by beam generated fusion reactions [31] which will result in there being an angular dependence in the neutron spectrum, it is important to apply this technique to the two (now three) side nTOF detectors, as well as the bottom two detectors, to better assess whether the neutrons observed are produced by a thermal plasma, beams, or a combination of the two.

These techniques could also be applied to other ICF facilities such as those at LLE [32] and LLNL [33]. This would include expanding the approach to include analyzing nTOF signals that measure the $14.1 \mathrm{MeV}$ neutrons from the reaction:

$$
D+T \rightarrow \propto(3.5 \mathrm{MeV})+n(14.1 \mathrm{MeV})
$$

This D-T reaction will be the reaction of choice for all ignition experiments since this reaction has the highest reaction cross section of all fusion reactions and the peak of the cross section occurs at the lowest ion energy. In addition to helping to analyze nTOF signals as on Z, an example of another potential application would be to help in the transfer of nTOF detector calibrations between facilities. For example, nTOF detectors have been calibrated at LLE for use on NIF. Since the scattering environment at LLE is 
not the same as at NIF, however, the transfer of the calibration is not straight forward. The use of the techniques described in this dissertation should be of great help in transferring these calibrations.

In addition to allowing the measurements of neutron yields, ion temperatures, and neutron spectra, nTOF detectors can be used to measure another extremely important parameter in inertial confinement fusion experiments: the $\rho R$ of the fuel $(\mathrm{g} / \mathrm{cm} 2)$. Here $\rho$ is the fuel density $\left(\mathrm{g} / \mathrm{cm}^{3}\right)$ and $\mathrm{R}$ is the radius of fuel $(\mathrm{cm})$, which is assumed to be spherical. For D-T fuel, the optimum value of $\rho R$ is $\sim 3 g / \mathrm{cm}^{2}$ [34]. For low values of $\rho R$, disassembly of the pellet becomes an issue, and for high values of $\rho R$, fuel depletion becomes an issue. For fusion to be an energetically viable energy source most of the D-T fuel must be heated, not by the laser driver (for example), but by the fusion reactions themselves. Since most neutrons escape with little or no interactions, this self-heating of the fuel will rely on the energy deposition of the $3.5 \mathrm{MeV}$ alpha particles in the fuel. It is envisioned that the laser will create a hot spot in the central core which ignites the fuel and that the resulting alphas will create a "burn wave" that propagates outward through cold fuel. For typical fuel masses, the $\rho$ R must be increased by a factor of about twenty over "normal", solid D-T values to simply support a burn propagation wave and over a factor of one hundred to attain optimal burn conditions (This later condition corresponds to increasing the fuel density by about a factor of a thousand over solid density). Thus, the $\rho$ R attained in a pellet implosion is an extremely critical measure of pellet performance [35]. 
Unfortunately, $\rho R$ is a difficult quantity to measure. One potential way to measure the $\rho R$ of D-T fuel is to measure the neutron "down scattered fraction" or dsf. Most of the $14.1 \mathrm{MeV}$ neutrons born in the fusion reactions escape the fuel without interacting. If high $\rho$ Rs are attained, however, a small fraction of the neutrons will down-scatter in the fuel and exit the fuel with energies lower than the initial $14.1 \mathrm{MeV}$ that they are born with. The fraction of scattered neutrons observed will be a measure of the fuel $\rho R$, so if the $d s f$ can be measured, the fuel $\rho R$ can be calculated. Since the scattered neutrons have less energy, they will travel more slowly to the nTOF detector so the detector's response to these neutrons will be separated in time from those of the primary pulse which will allow their measurement. However, the fact that the scattered neutrons will have lower energies also means that, neutron for neutron, they will induce less light output in the nTOF detector. Thus, to get the true $d s f$ the light output of the respective signals must be adjusted for neutron energy - something that can be readily accomplished by the use of the techniques described herein.

These techniques can also be applied to $\rho R$ measurements of $D-D$ fusion experiments. In the case of D-D fusion there are two reactions of roughly equal probability:

$$
\begin{aligned}
& D+D \rightarrow \mathrm{He}^{3}(0.82 \mathrm{MeV})+n(2.45 \mathrm{MeV}) \\
& D+D \rightarrow T(1.01 \mathrm{MeV})+p(3.04 \mathrm{MeV})
\end{aligned}
$$

The product tritium of equation 36 can react with the deuterium fuel and drive the D-T reaction above (equation 34). The ratio of D-D to D-T reactions is a function of the $\rho R$ of the fuel, so measuring the ratio of D-D to D-T neutrons will give a measure of the fuel 
$\rho R$. As above, the difference in energies of the $2.45 \mathrm{MeV} D-D$ neutrons and the 14.1 MeV D-T neutrons means that the two signals will be well-separated in time at an nTOF detector. Again, by properly adjusting the light output of the two signals for the different neutron energies using the techniques described in this dissertation, the ratio of D-D to D-T reactions can be measured, which, in turn will yield the fuel $\rho R$.

ICF applications are also requiring scintillators with ever-faster time responses. This need leads to the introduction of novel scintillation materials. For example, the primary $d s f$ nTOF detector at NIF uses xylene as the scintillation material. This material (or other "exotic" materials that might be used) may not have the same light output curve as typical plastic scintillators (equations 2 and 3, page 10; also Figure 7, page 11). Thus, to fully generalize the techniques discussed herein will require the experimental verification of the light output curves of all the scintillation materials being used.

The technique described herein has also been used to model the effectiveness of a new collimator design. It has been shown that the addition of a collimator did indeed improve the neutron signals but there was still room for improvement. Therefore, a new collimator design was undertaken, to be more massive than the first, and was shown with modeling that it was much more effective at eliminating neutrons that would contribute to scattering into the detectors later in time. This is shown in full in Appendix F. 
APPENDICIES 


\section{APPENDIX A}

\section{MCNP-PoliMi INPUT DECK}

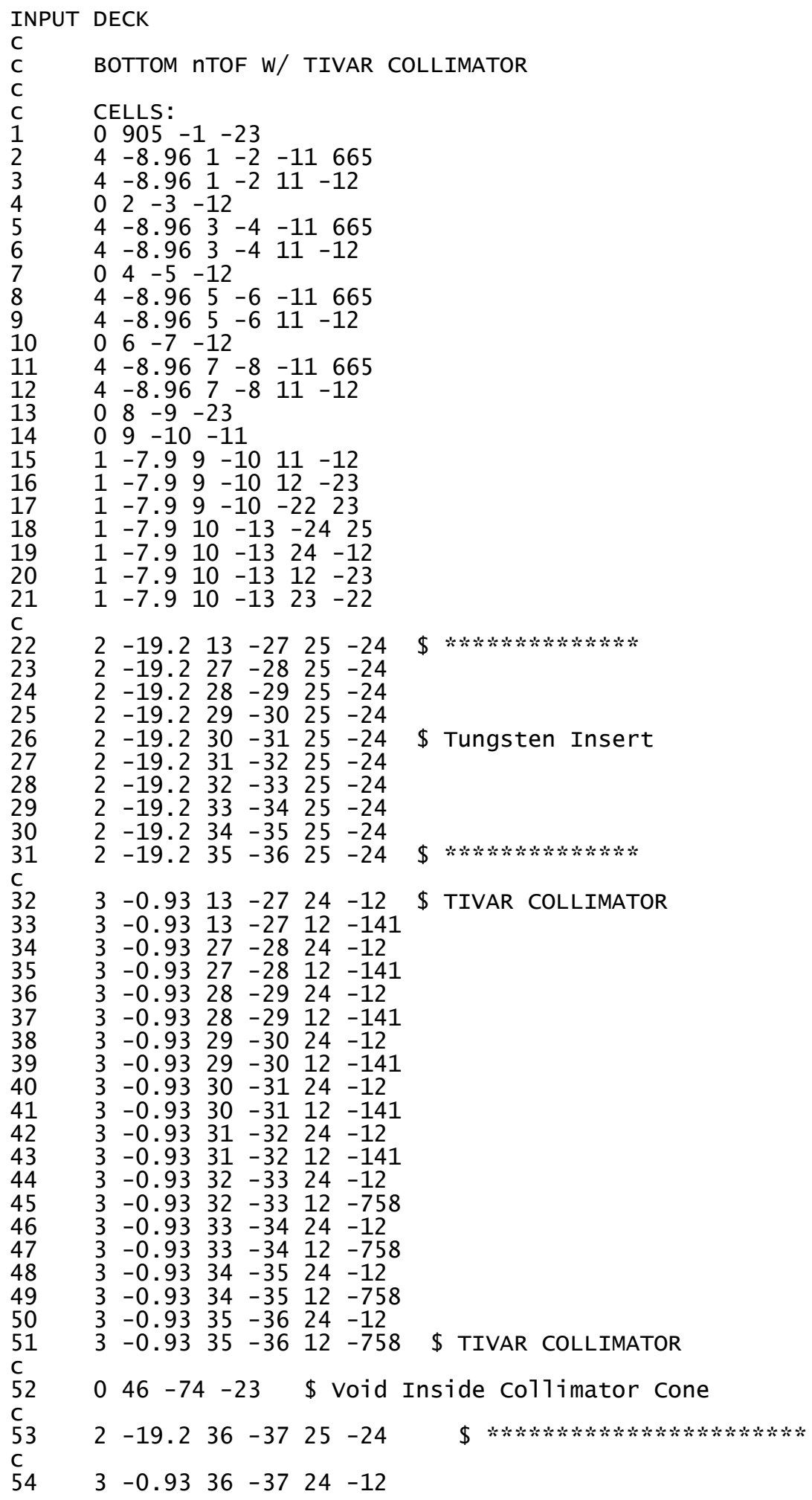




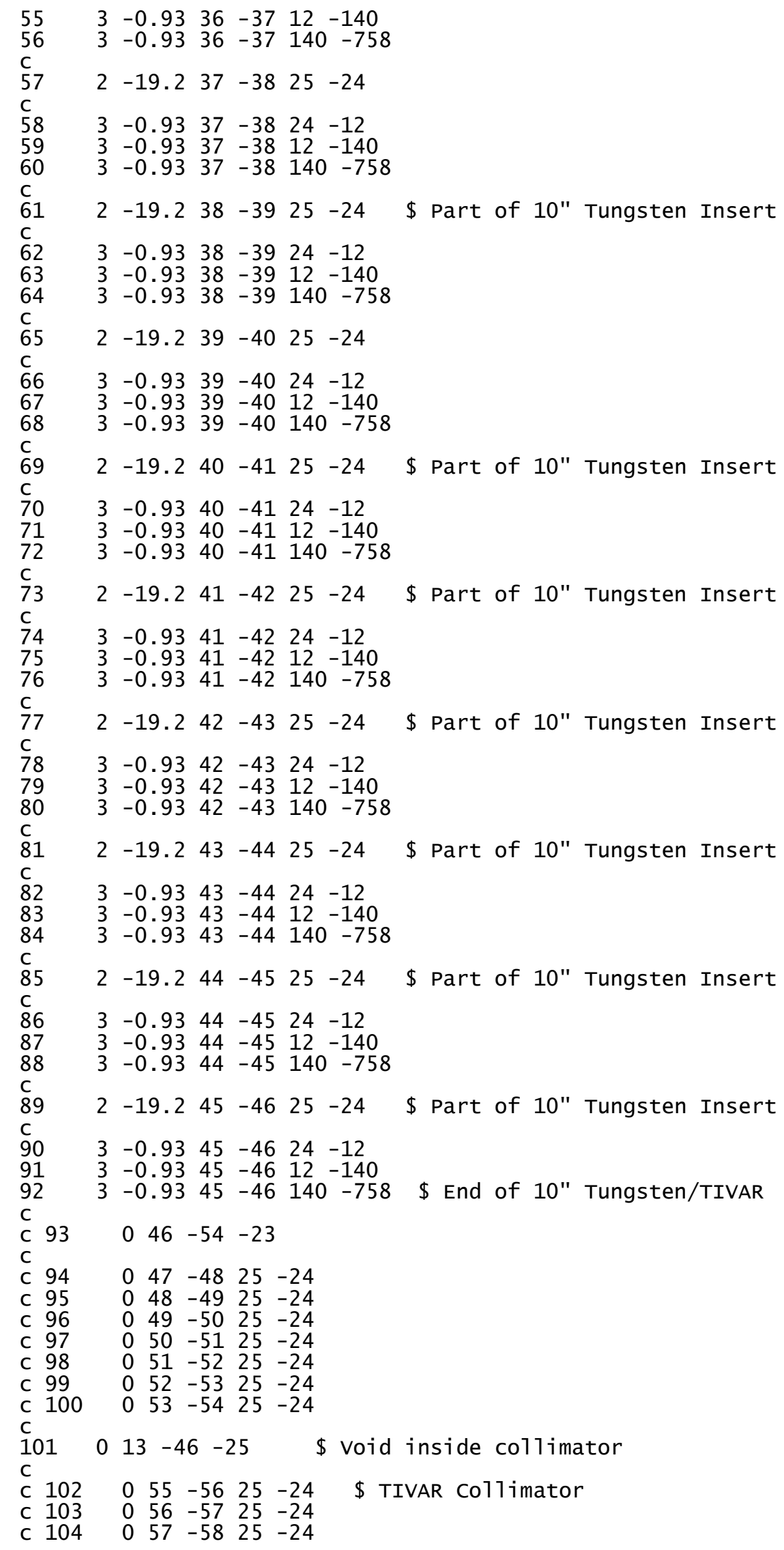




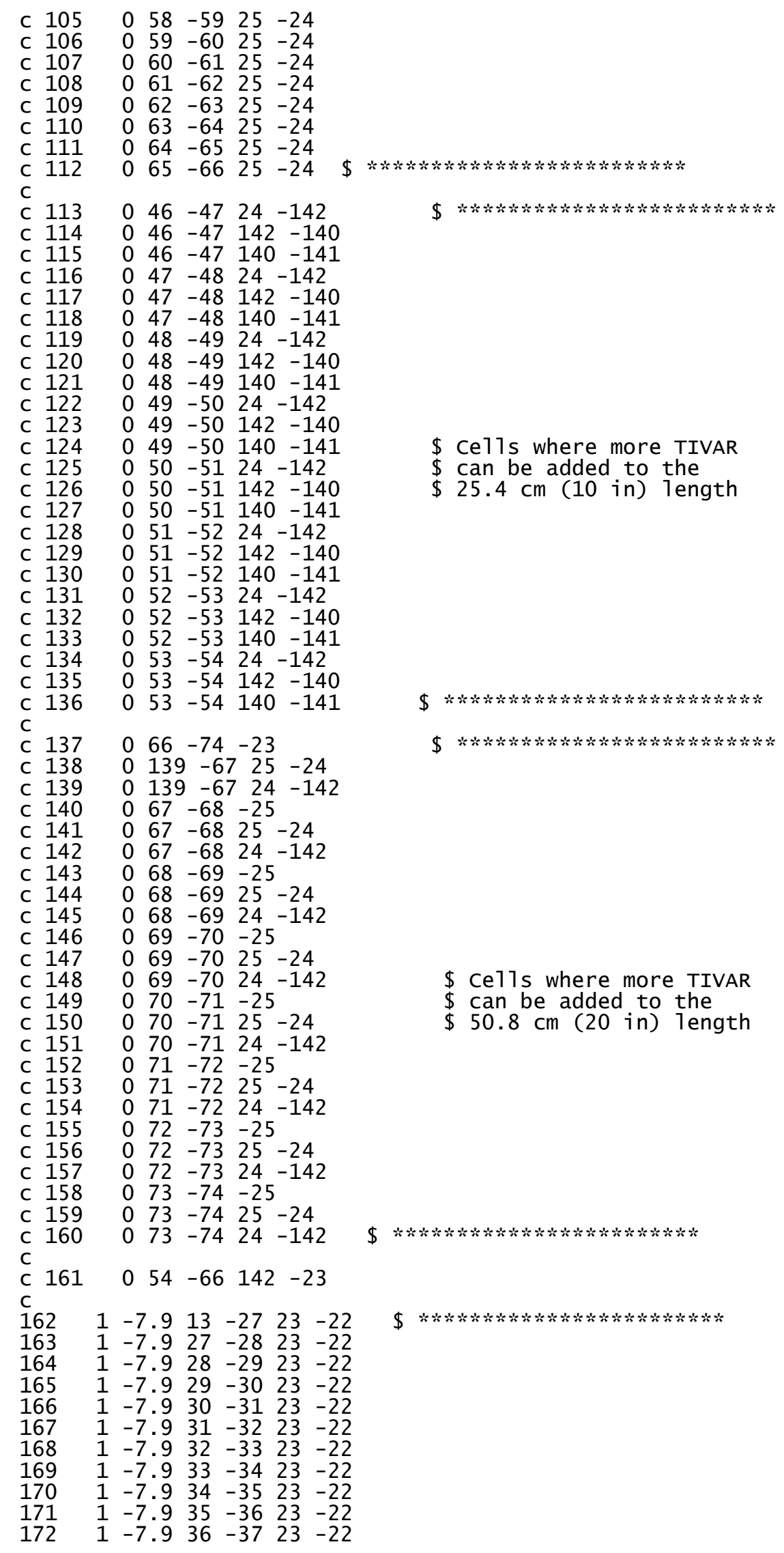




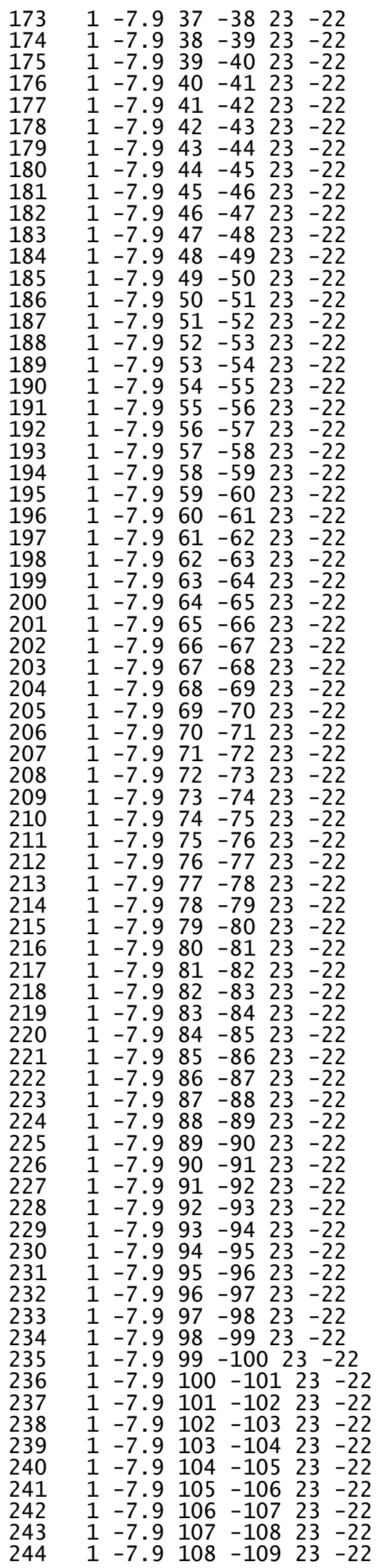

\$ MITL Cone 


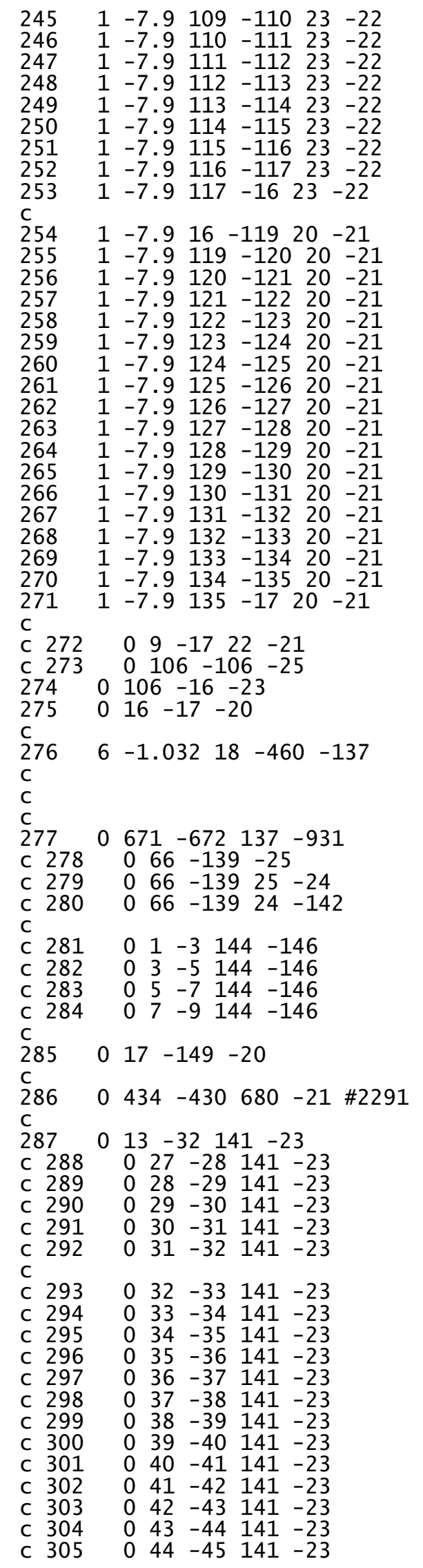

$\$ * * * * * * * * * * * * * * * * * * * * * * * * * * * * * * * * *$

$\$ * * * * * * * * * * * * * * * * * * * * * * * * * * * * * * * * *$

\$ Bottom Cylinder of MITLs

\$ $* * * * * * * * * * * * * * * * * * * * * * * * * * * * * * * * *$

\$ Don't need -- see ce11 481

$\$$ Inside Col1imator Cone (25)

\$ TOP nTOF Scintillator Ce11 Either BC-418 or BC-422Q -Same Density and Ratio

$\$$ Pig Cel1

\$ Tal1y Ce11 at Col1imator Exit

$\$ * * * * * * * * * * * * * * * * * * * * * * * *$

$\$$ Void Cells around outside

$\$$ of MITL Cone (22 \& 23)

$\$ * * * * * * * * * * * * * * * * * * * * * * * * *$ 


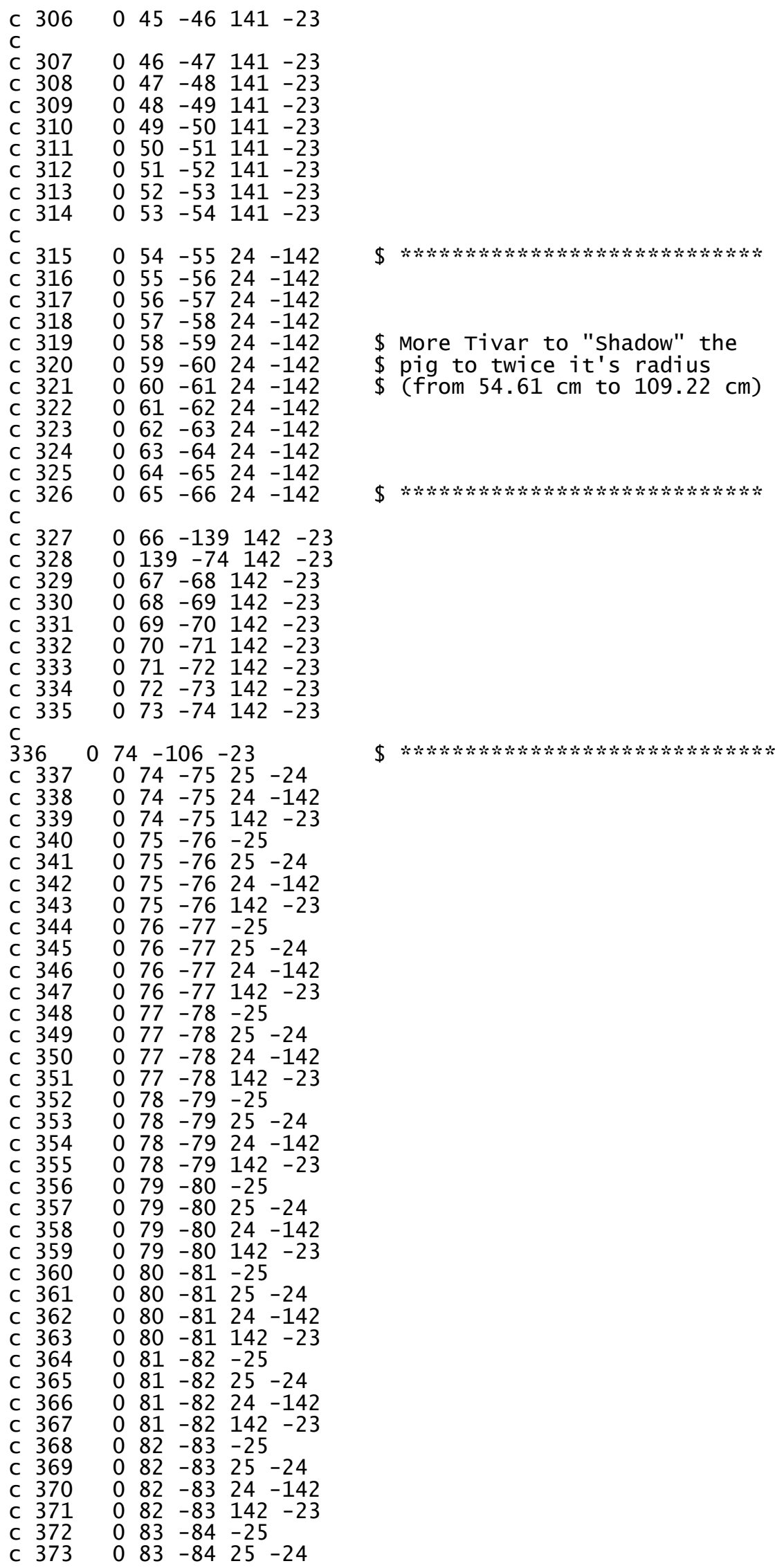




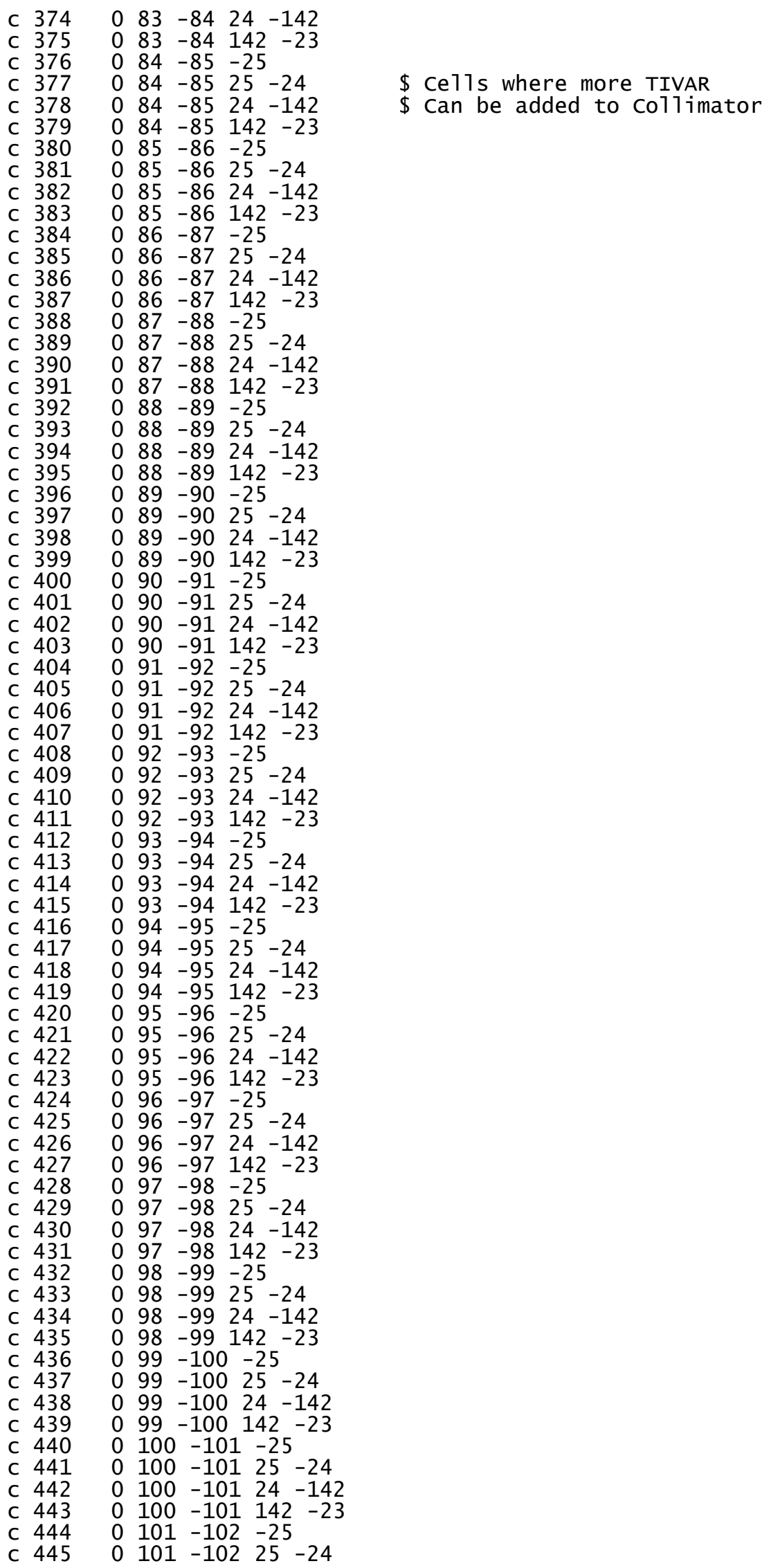




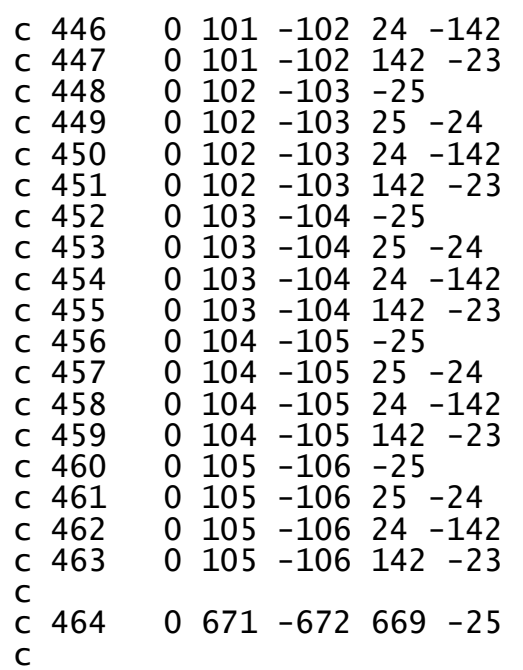

$\begin{array}{llllllll}465 & 1 & -7.9 & 1 & -2 & 23 & -22\end{array}$

$\begin{array}{lllllll}466 & 1 & -7.9 & 2 & -3 & 23 & -22\end{array}$

$\begin{array}{lllllll}467 & 1 & -7.9 & 3 & -4 & 23 & -22\end{array}$

$\begin{array}{lllllll}468 & 1 & -7.9 & 4 & -5 & 23 & -22\end{array}$

$\begin{array}{lllllll}469 & 1 & -7.9 & 5 & -6 & 23 & -22\end{array}$

$\begin{array}{lllllll}470 & 1 & -7.9 & 6 & -7 & 23 & -22\end{array}$

$\begin{array}{lllllll}471 & 1 & -7.9 & 7 & -8 & 23 & -22\end{array}$

$\begin{array}{llllllll}472 & 1 & -7.9 & 8 & -9 & 23 & -22\end{array}$

C

$\begin{array}{llllll}473 & 0 & 1 & -8 & 12 & -23\end{array}$

C

$\begin{array}{lllllll}\text { C } & 474 & 0 & 3 & -4 & 12 & -23\end{array}$

$\begin{array}{llllll}\text { C } 475 & 0 & 5 & -6 & 12 & -23\end{array}$

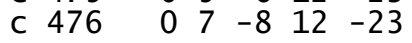

C

$\begin{array}{lllllll}\text { C } & 477 & 0 & 2 & -3 & 22 & -143\end{array}$

$\begin{array}{llllll}\text { C } 478 & 0 & 4 & -5 & 22 & -143\end{array}$

$\begin{array}{llllll}\text { C } 479 & 0 & 6 & -7 & 22 & -143\end{array}$

$\begin{array}{lllllll}\text { C } & 480 & 0 & 8 & -9 & 22 & -143\end{array}$

C

$\begin{array}{llllll}481 & 0 & 909 & -145 & 144 & -146\end{array}$

$\begin{array}{llllll}482 & 0 & 908 & -16 & 22 & -143\end{array}$

$\begin{array}{llllll}483 & 0 & 16 & -145 & 21 & -143\end{array}$

$\begin{array}{llllll}484 & 0 & 145 & -149 & 21 & -146\end{array}$

$48500149-675-146$

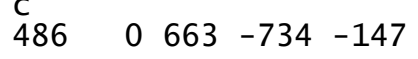

C

$\begin{array}{llllllll}487 & 1 & -7.9 & 1 & -2 & 143 & -144\end{array}$

$\begin{array}{lllllll}488 & 1 & -7.9 & 2 & -3 & 143 & -144\end{array}$

$\begin{array}{lllllll}489 & 1 & -7.9 & 3 & -4 & 143 & -144\end{array}$

$\begin{array}{lllllll}490 & 1 & -7.9 & 4 & -5 & 143 & -144\end{array}$

$\begin{array}{lllllll}491 & 1 & -7.9 & 5 & -6 & 143 & -144\end{array}$

$\begin{array}{lllllll}492 & 1 & -7.9 & 6 & -7 & 143 & -144\end{array}$

$\begin{array}{lllllll}493 & 1 & -7.9 & 7 & -8 & 143 & -144\end{array}$

$\begin{array}{lllllll}494 & 1 & -7.9 & 8 & -9 & 143 & -144\end{array}$

$\begin{array}{lllllll}495 & 1 & -7.9 & 9 & -10 & 143 & -144\end{array}$

$\begin{array}{lllllll}496 & 1 & -7.9 & 10 & -13 & 143 & -144\end{array}$

$\begin{array}{lllllll}497 & 1 & -7.9 & 13 & -27 & 143 & -144\end{array}$

$\begin{array}{lllllll}498 & 1 & -7.9 & 27 & -28 & 143 & -144\end{array}$

$\begin{array}{lllllll}499 & 1 & -7.9 & 28 & -29 & 143 & -144\end{array}$

$\begin{array}{lllllll}500 & 1 & -7.9 & 29 & -30 & 143 & -144\end{array}$

$\begin{array}{lllllll}501 & 1 & -7.9 & 30 & -31 & 143 & -144\end{array}$

$\begin{array}{lllllll}502 & 1 & -7.9 & 31 & -32 & 143 & -144\end{array}$

$\begin{array}{lllllll}503 & 1 & -7.9 & 32 & -33 & 143 & -144\end{array}$

$\begin{array}{lllllll}504 & 1 & -7.9 & 33 & -34 & 143 & -144\end{array}$

$\begin{array}{lllllll}505 & 1 & -7.9 & 34 & -35 & 143 & -144\end{array}$

$\begin{array}{lllllll}506 & 1 & -7.9 & 35 & -36 & 143 & -144\end{array}$

$\begin{array}{lllllll}507 & 1 & -7.9 & 36 & -37 & 143 & -144\end{array}$

$\begin{array}{lllllll}508 & 1 & -7.9 & 37 & -38 & 143 & -144\end{array}$

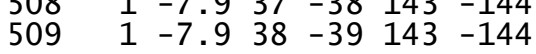

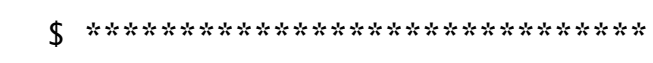

\$ Ring around Scintillator the size

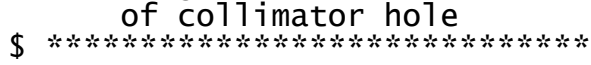

$\$ * * * * * * * * * * * * * * * * * * * * * * * * * * * * *$

$\$$ Void Cells on inside top

$\$$ of MITL Cone (22 \& 23)

$\$$ Void Cells on outside top

$\$$ of MITL Cone (22 \& 23)

$\$$ Void b/w 2nd MITL Cone and stack

$\$$ void b/w 1st \& 2nd MITL Cone

$\$$ void b/w MITL Cy $1 \# 1$ and stack $\$$ void b/w MITL \#1 \& Bottom of stack

$\$$ Void b/w Stack and Detector

$\$ * * * * * * * * * * * * * * * * * * * * * * * * * * * * * * *$ 


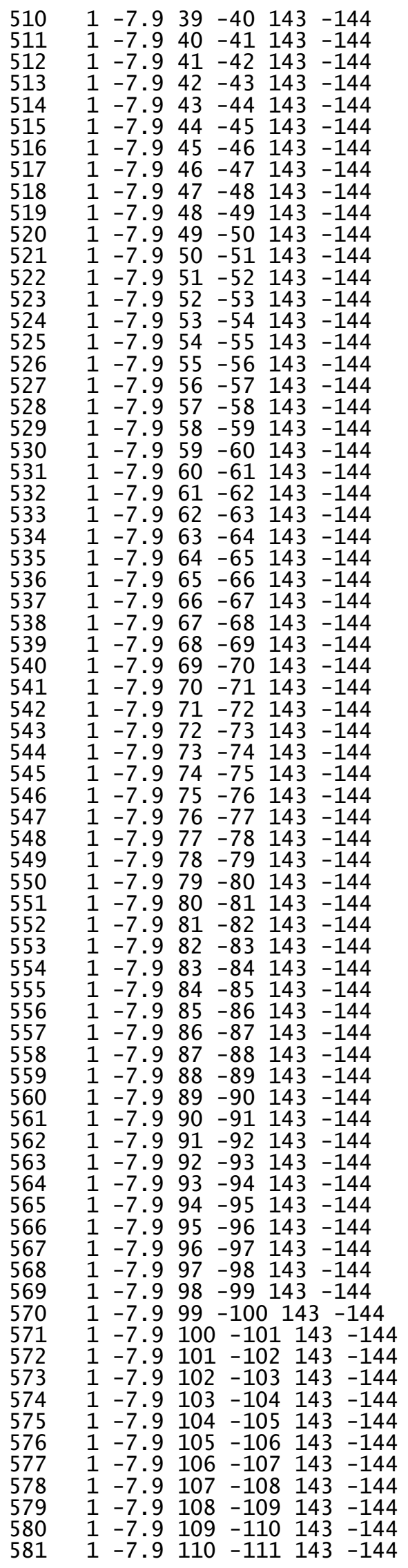




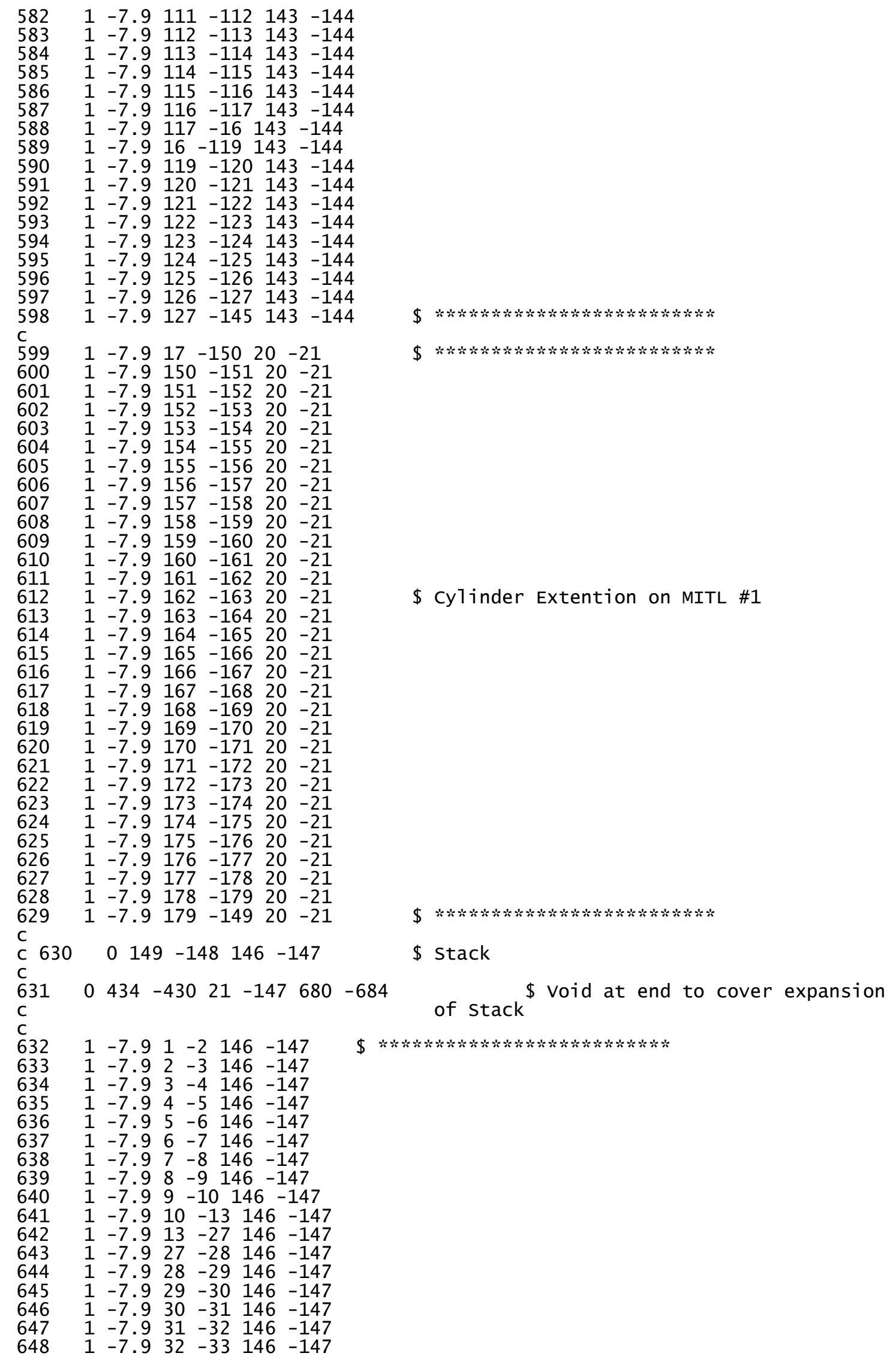




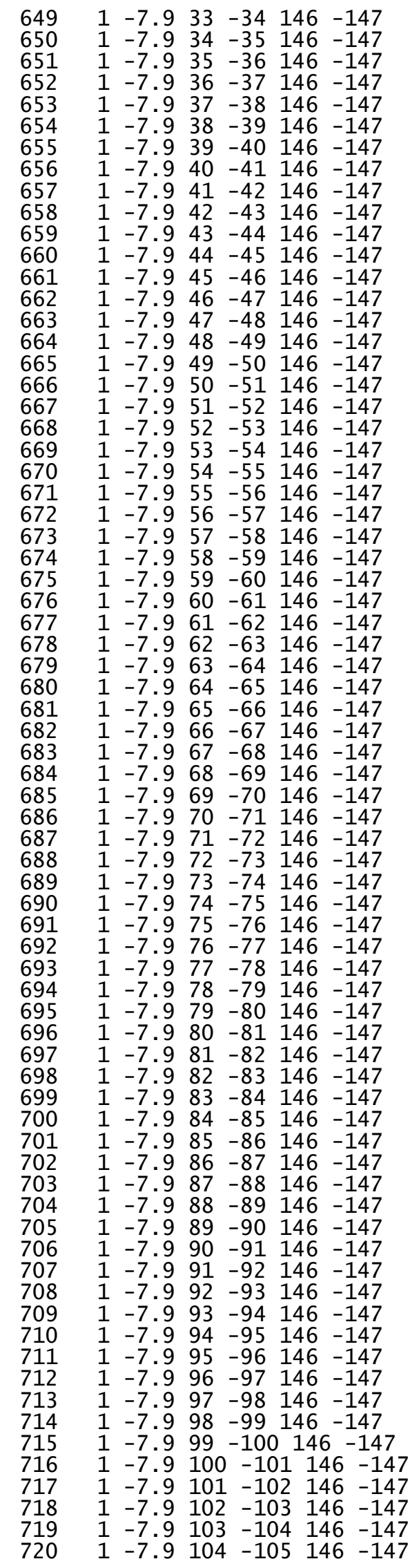




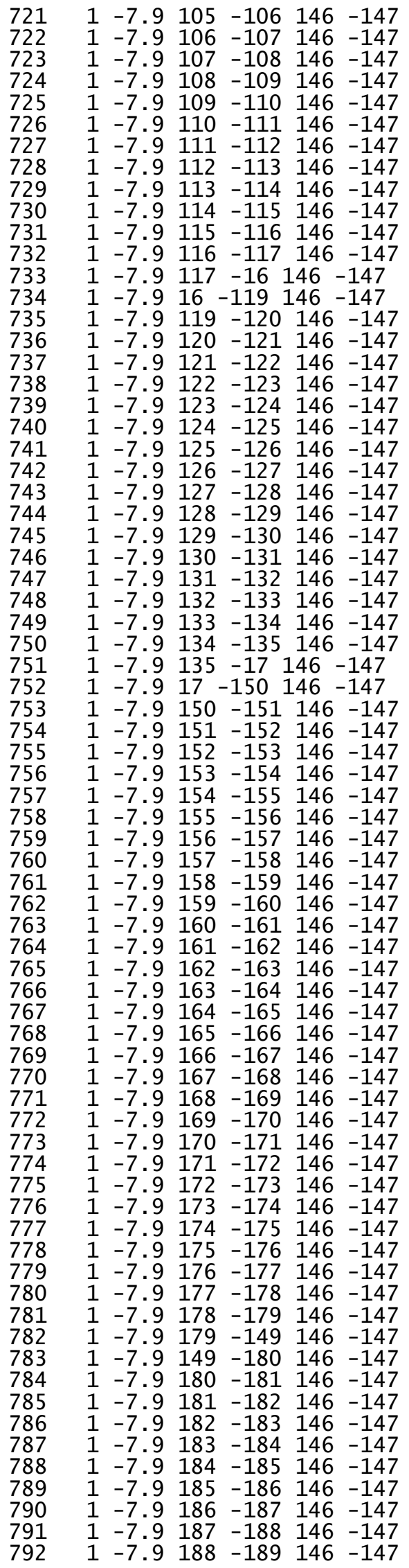




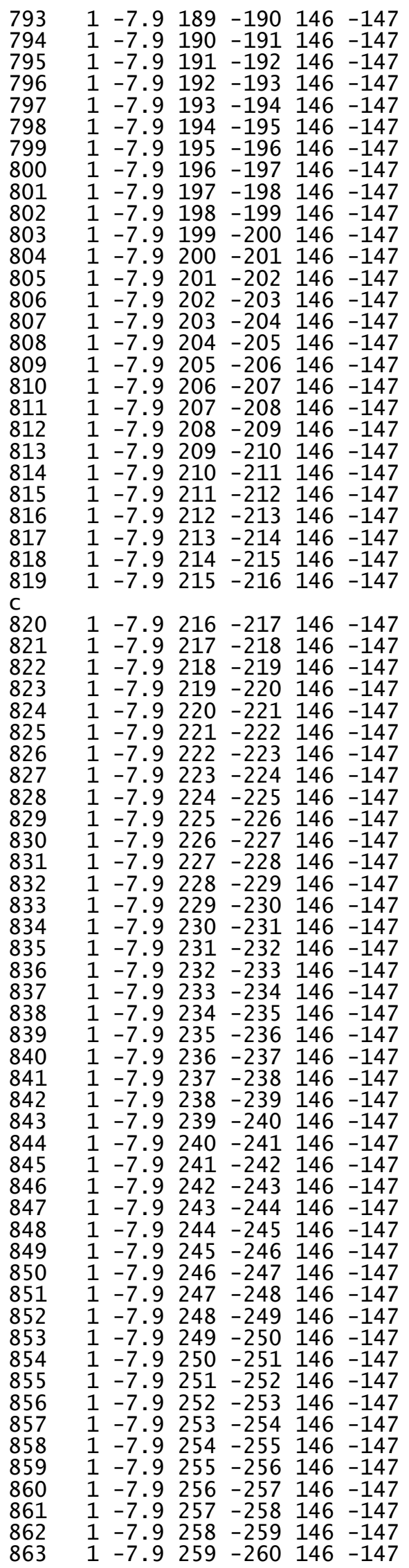




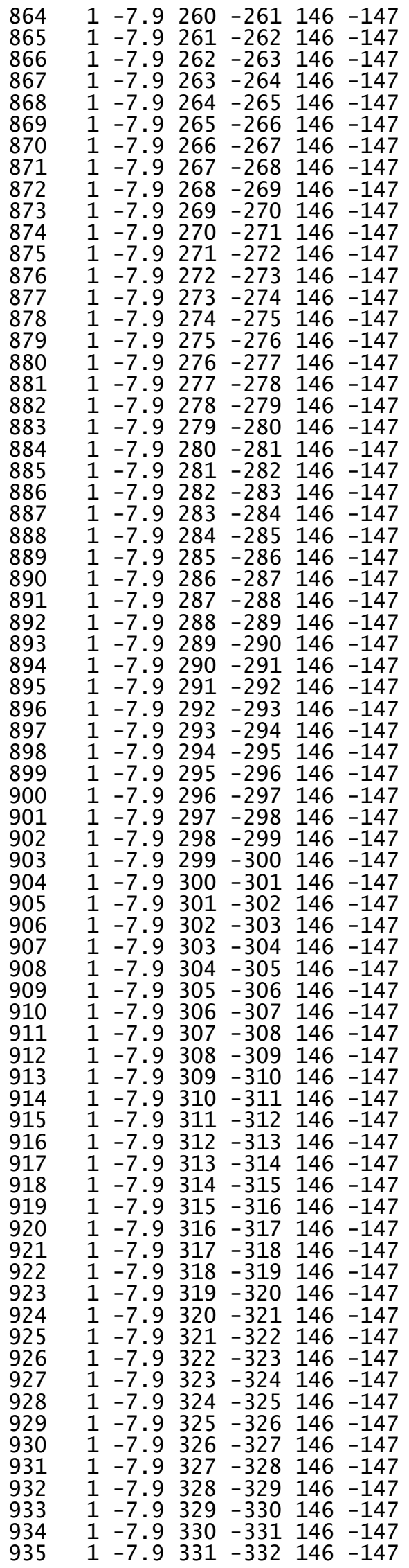




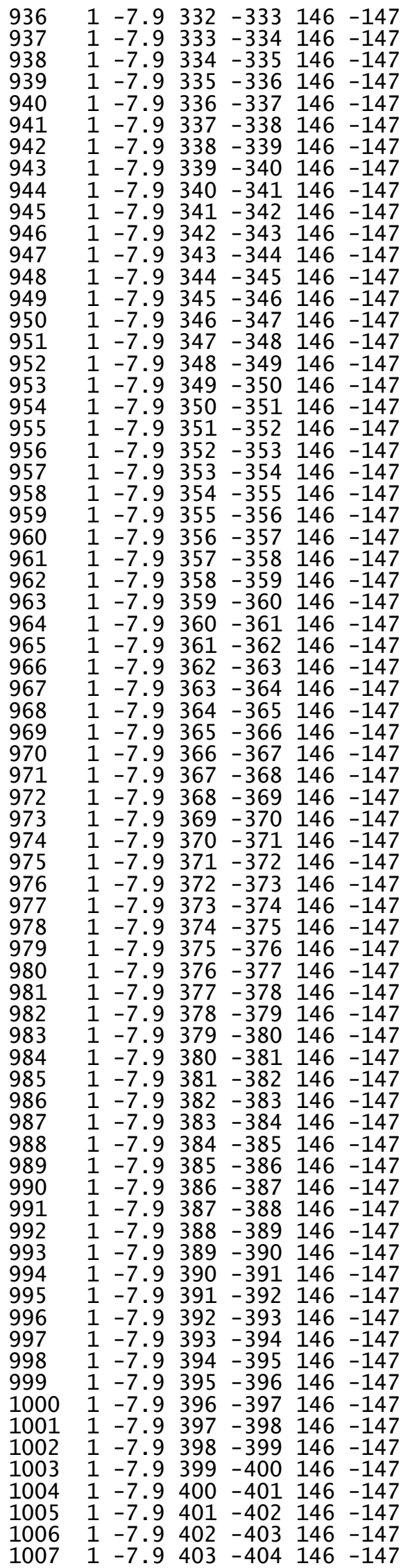




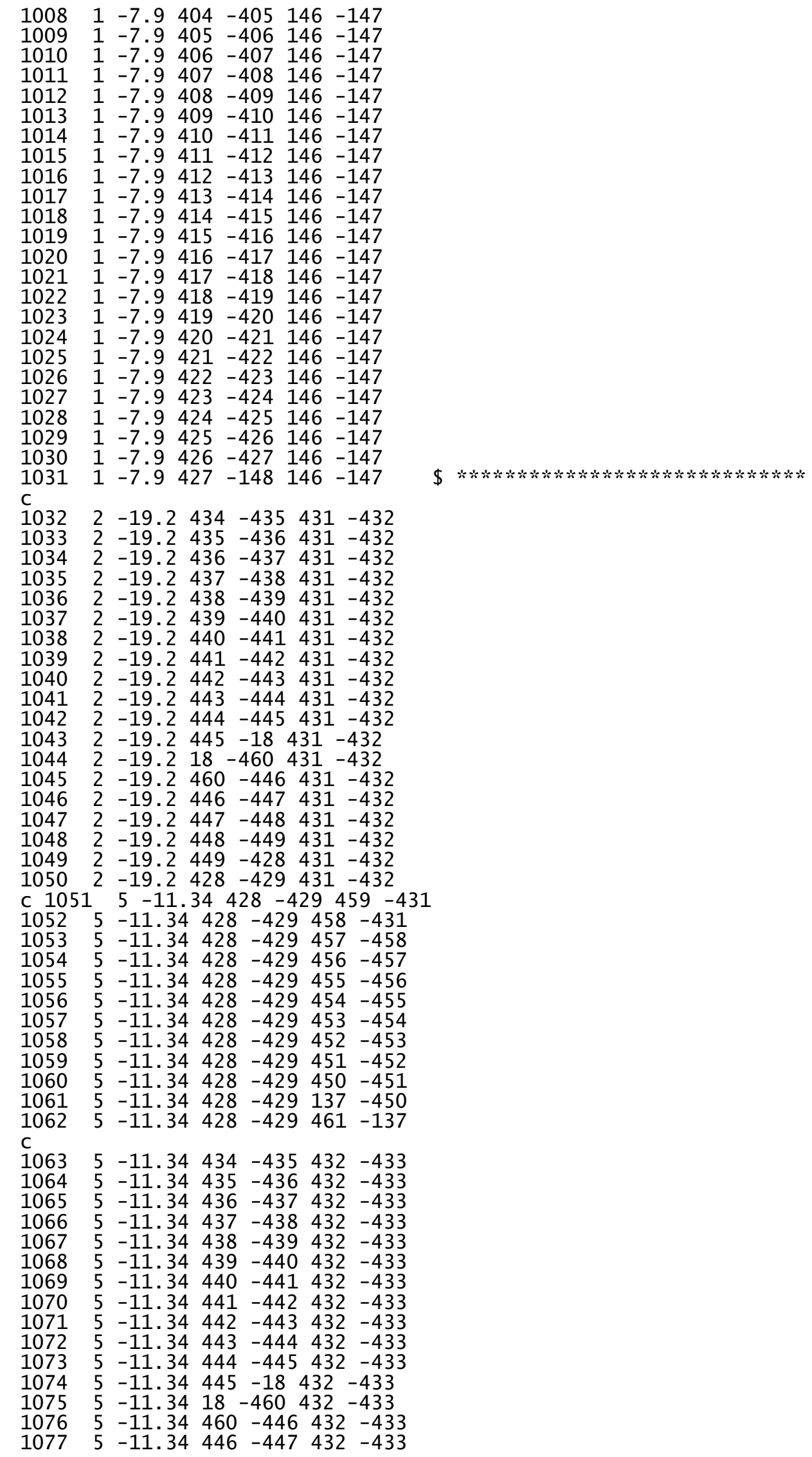




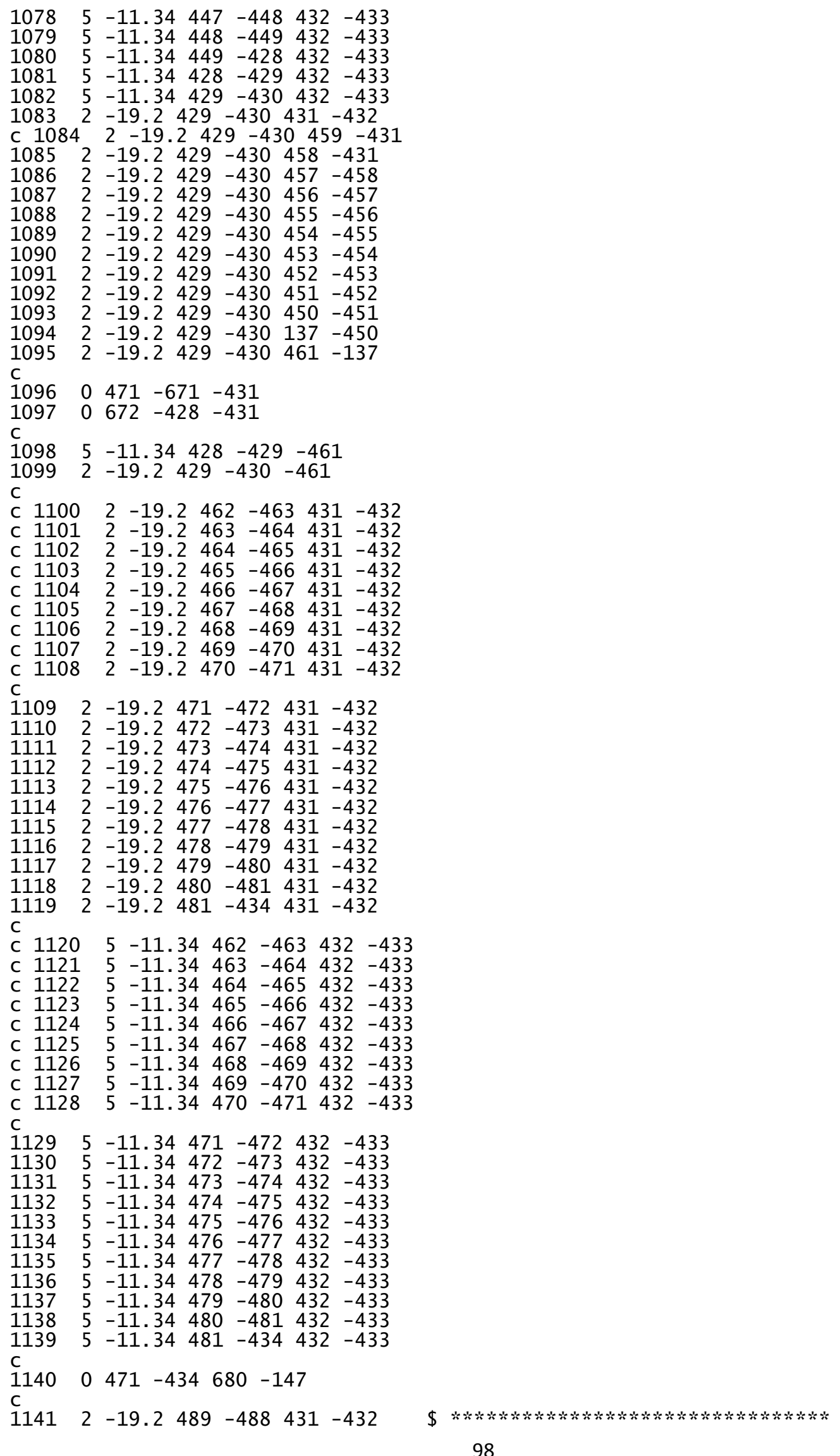




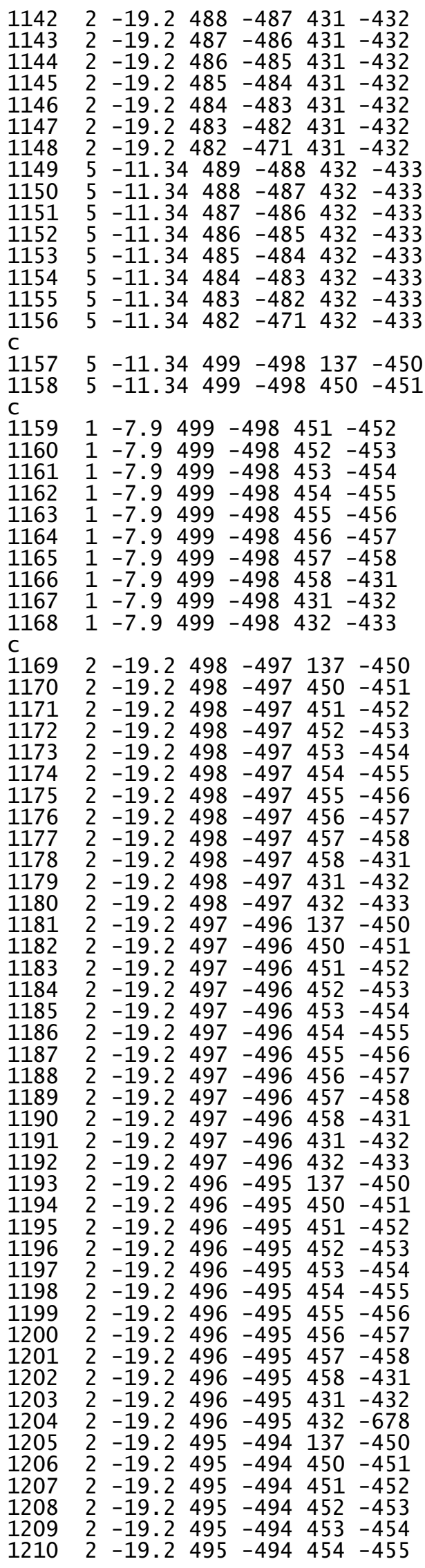

$\$$ Pig made 8" longer for a total $\$$ length of 48 "

$\$ * * * * * * * * * * * * * * * * * * * * * * * * * * * * * * * *$

$\$ * * * * * * * * * * * * * * * * * * * * * * * * * * * * * * * *$
\$ 10" tUNGSTEN PlUG WITH A 3" DIA hOLE 


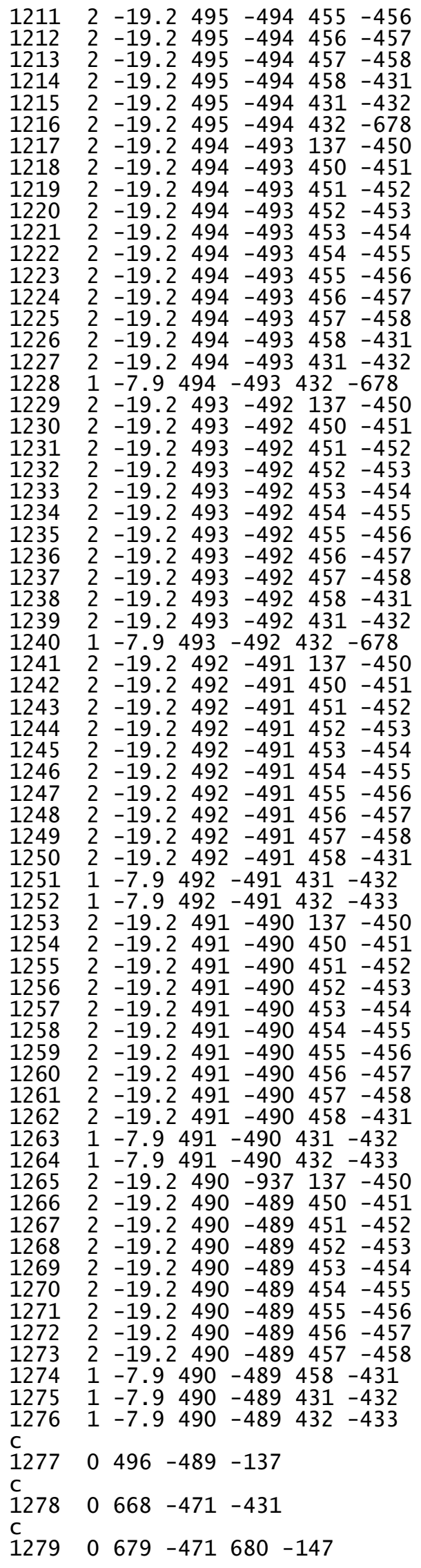

$\$ * * * * * * * * * * * * * * * * * * * * * * * * * * * * * * * * * * * * *$ \$3" diameter hole thru Tungsten Plug $\$$ Void on inside of Pig $\$$ void on outside of Pig 100 


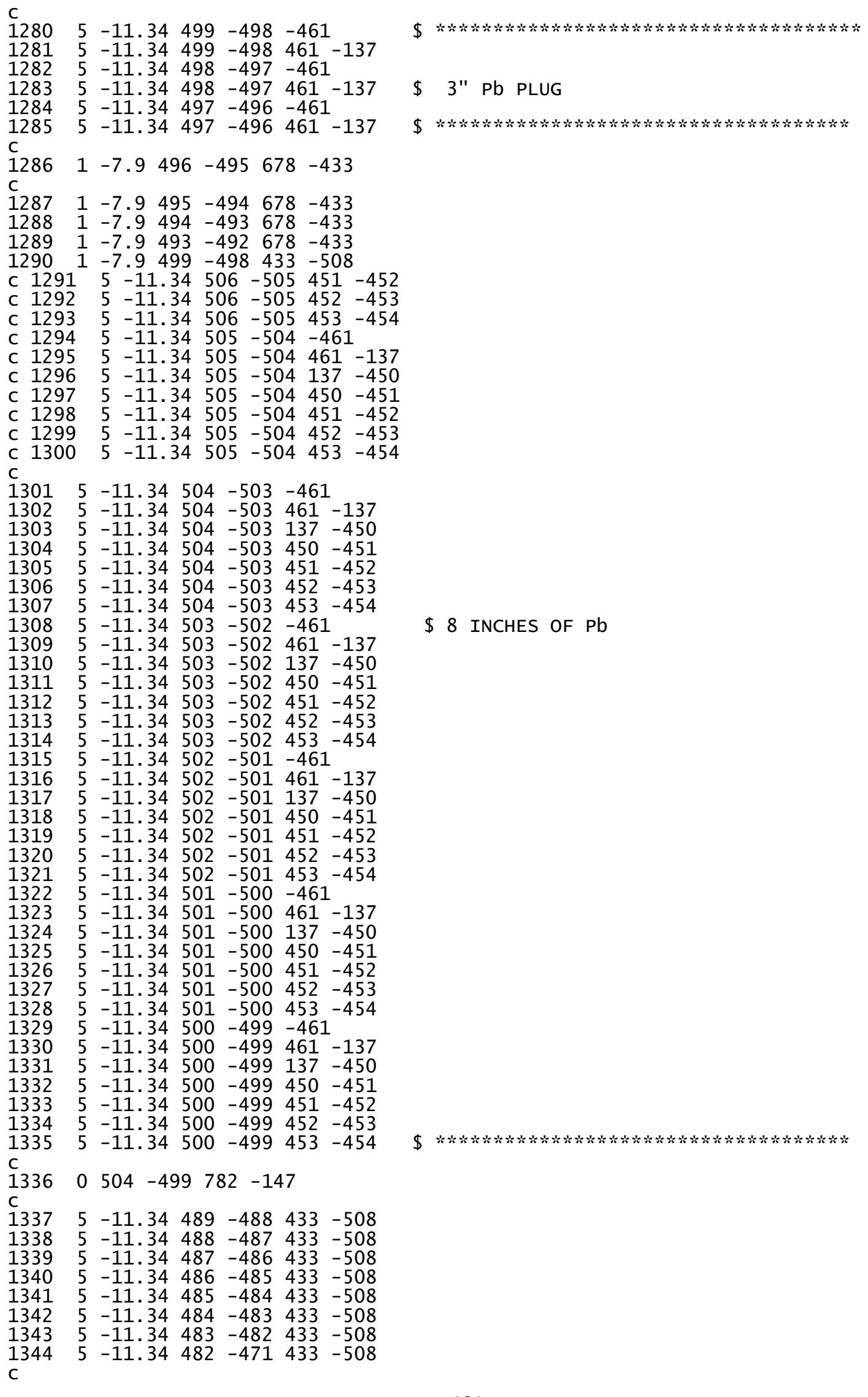




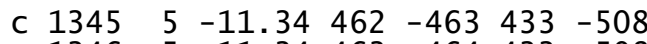

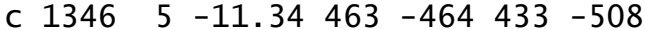

$$
\begin{aligned}
& \begin{array}{lllllll}
\text { C } 1347 & 5 & -11.34 & 464 & -465 & 433 & -508
\end{array} \\
& \begin{array}{llllllll}
\text { C } & 1348 & 5 & -11.34 & 465 & -466 & 433 & -508
\end{array} \\
& \begin{array}{llllllll}
\text { C } & 1349 & 5 & -11.34 & 466 & -467 & 433 & -508
\end{array}
\end{aligned}
$$

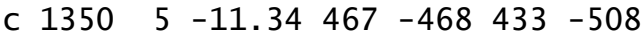

$$
\begin{aligned}
& \begin{array}{lllllll}
\text { C } 1351 & 5 & -11.34 & 468 & -469 & 433 & -508
\end{array} \\
& \begin{array}{lllllll}
\text { C } 1352 & 5 & -11.34 & 469 & -470 & 433 & -508
\end{array} \\
& \begin{array}{lllllll}
\text { C } 1353 & 5 & -11.34 & 470 & -471 & 433 & -508
\end{array} \\
& \text { c } \\
& \begin{array}{llllllll}
1354 & 5 & -11.34 & 471 & -472 & 433 & -508
\end{array} \\
& \begin{array}{lllllll}
1355 & 5 & -11.34 & 472 & -473 & 433 & -508
\end{array} \\
& \begin{array}{lllllll}
1356 & 5 & -11.34 & 473 & -474 & 433 & -508
\end{array} \\
& \begin{array}{lllllll}
1357 & 5 & -11.34 & 474 & -475 & 433 & -508
\end{array} \\
& \begin{array}{lllllll}
1358 & 5 & -11.34 & 475 & -476 & 433 & -508
\end{array} \\
& \begin{array}{lllllll}
1359 & 5 & -11.34 & 476 & -477 & 433 & -508
\end{array} \\
& \begin{array}{lllllll}
1360 & 5 & -11.34 & 477 & -478 & 433 & -508
\end{array} \\
& \begin{array}{lllllll}
1361 & 5 & -11.34 & 478 & -479 & 433 & -508
\end{array} \\
& \begin{array}{lllllll}
1362 & 5 & -11.34 & 479 & -480 & 433 & -508
\end{array} \\
& \begin{array}{lllllll}
1363 & 5 & -11.34 & 480 & -481 & 433 & -508
\end{array} \\
& \begin{array}{lllllll}
1364 & 5 & -11.34 & 481 & -434 & 433 & -508
\end{array} \\
& \begin{array}{lllllll}
1365 & 5 & -11.34 & 434 & -435 & 433 & -508
\end{array} \\
& \begin{array}{lllllll}
1366 & 5 & -11.34 & 435 & -436 & 433 & -508
\end{array} \\
& \begin{array}{lllllll}
1367 & 5 & -11.34 & 436 & -437 & 433 & -508
\end{array} \\
& \begin{array}{lllllll}
1368 & 5 & -11.34 & 437 & -438 & 433 & -508
\end{array} \\
& \begin{array}{lllllll}
1369 & 5 & -11.34 & 438 & -439 & 433 & -508
\end{array} \\
& \begin{array}{lllllll}
1370 & 5 & -11.34 & 439 & -440 & 433 & -508
\end{array} \\
& \begin{array}{lllllll}
1371 & 5 & -11.34 & 440 & -441 & 433 & -508
\end{array} \\
& \begin{array}{lllllll}
1372 & 5 & -11.34 & 441 & -442 & 433 & -508
\end{array} \\
& \begin{array}{lllllll}
1373 & 5 & -11.34 & 442 & -443 & 433 & -508
\end{array} \\
& \begin{array}{lllllll}
1374 & 5 & -11.34 & 443 & -444 & 433 & -508
\end{array} \\
& \begin{array}{lllllll}
1375 & 5 & -11.34 & 444 & -445 & 433 & -508
\end{array} \\
& \begin{array}{lllllll}
1376 & 5 & -11.34 & 445 & -18 & 433 & -508
\end{array} \\
& \begin{array}{lllllll}
1377 & 5 & -11.34 & 18 & -460 & 433 & -508
\end{array} \\
& \begin{array}{lllllll}
1378 & 5 & -11.34 & 460 & -446 & 433 & -508
\end{array} \\
& \begin{array}{lllllll}
1379 & 5 & -11.34 & 446 & -447 & 433 & -508
\end{array} \\
& \begin{array}{lllllll}
1380 & 5 & -11.34 & 447 & -448 & 433 & -508
\end{array} \\
& \begin{array}{lllllll}
1381 & 5 & -11.34 & 448 & -449 & 433 & -508
\end{array} \\
& \begin{array}{lllllll}
1382 & 5 & -11.34 & 449 & -428 & 433 & -508
\end{array} \\
& \begin{array}{lllllll}
1383 & 5 & -11.34 & 428 & -429 & 433 & -508
\end{array} \\
& \begin{array}{lllllll}
1384 & 5 & -11.34 & 429 & -430 & 433 & -508
\end{array}
\end{aligned}
$$




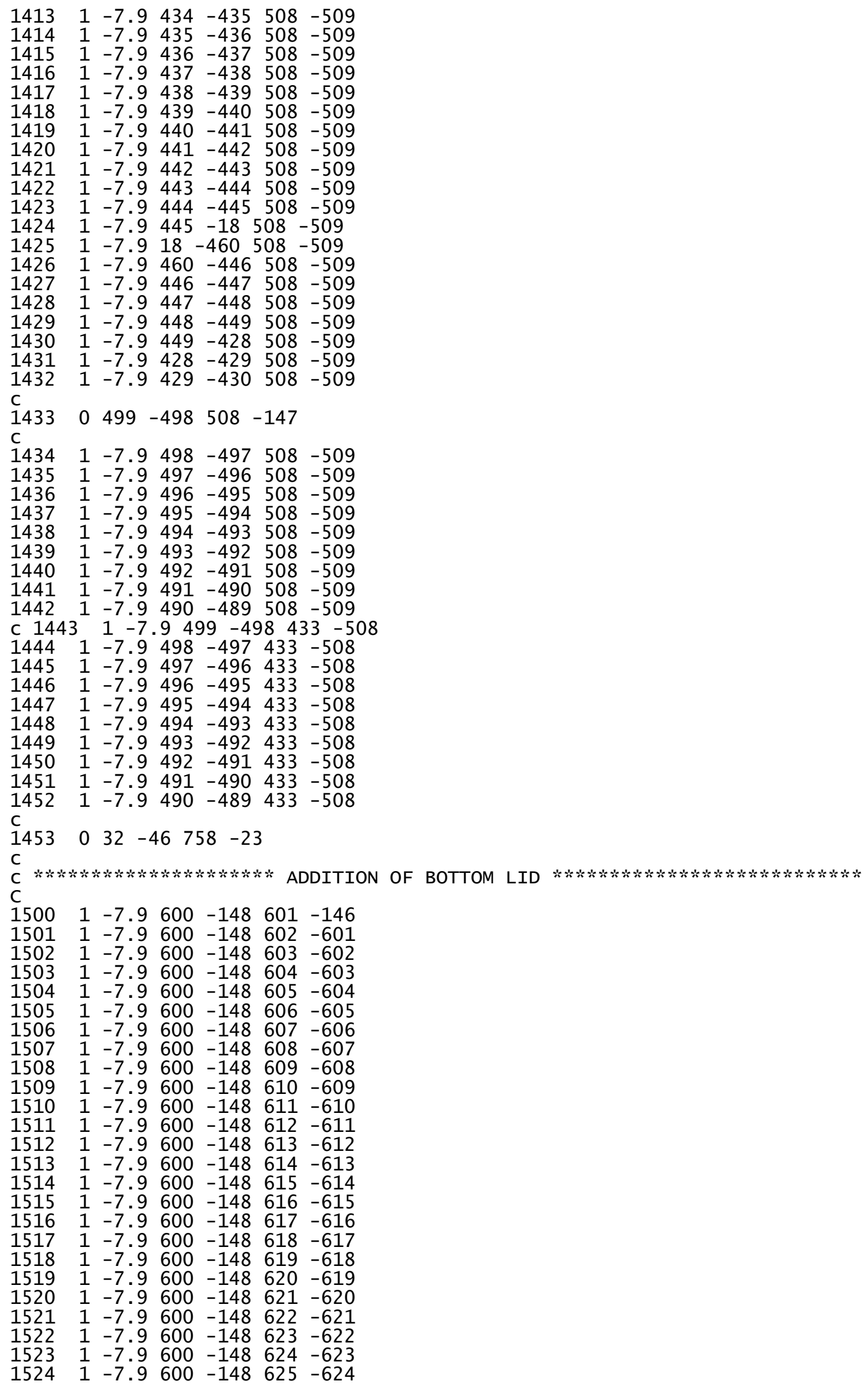




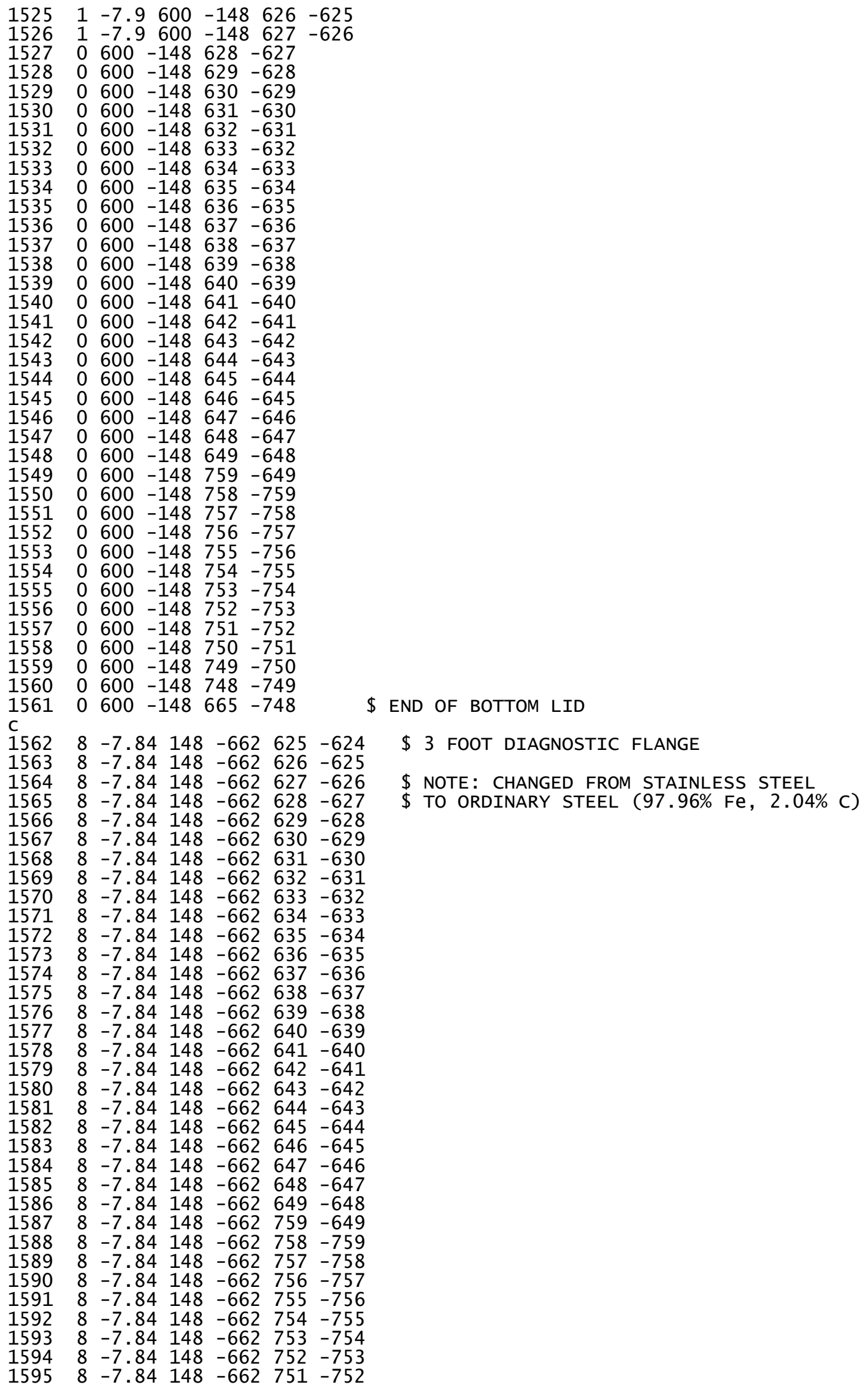




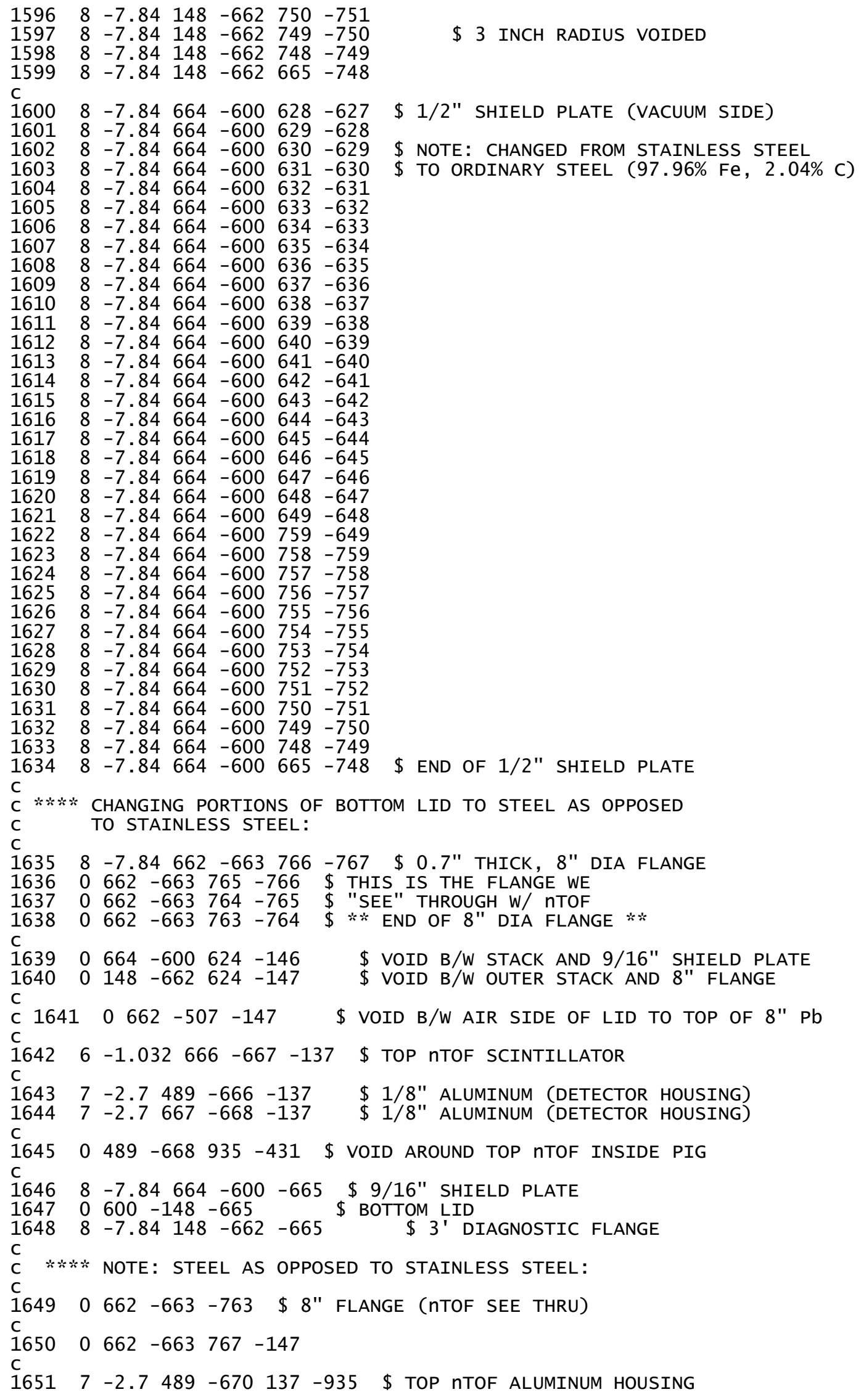




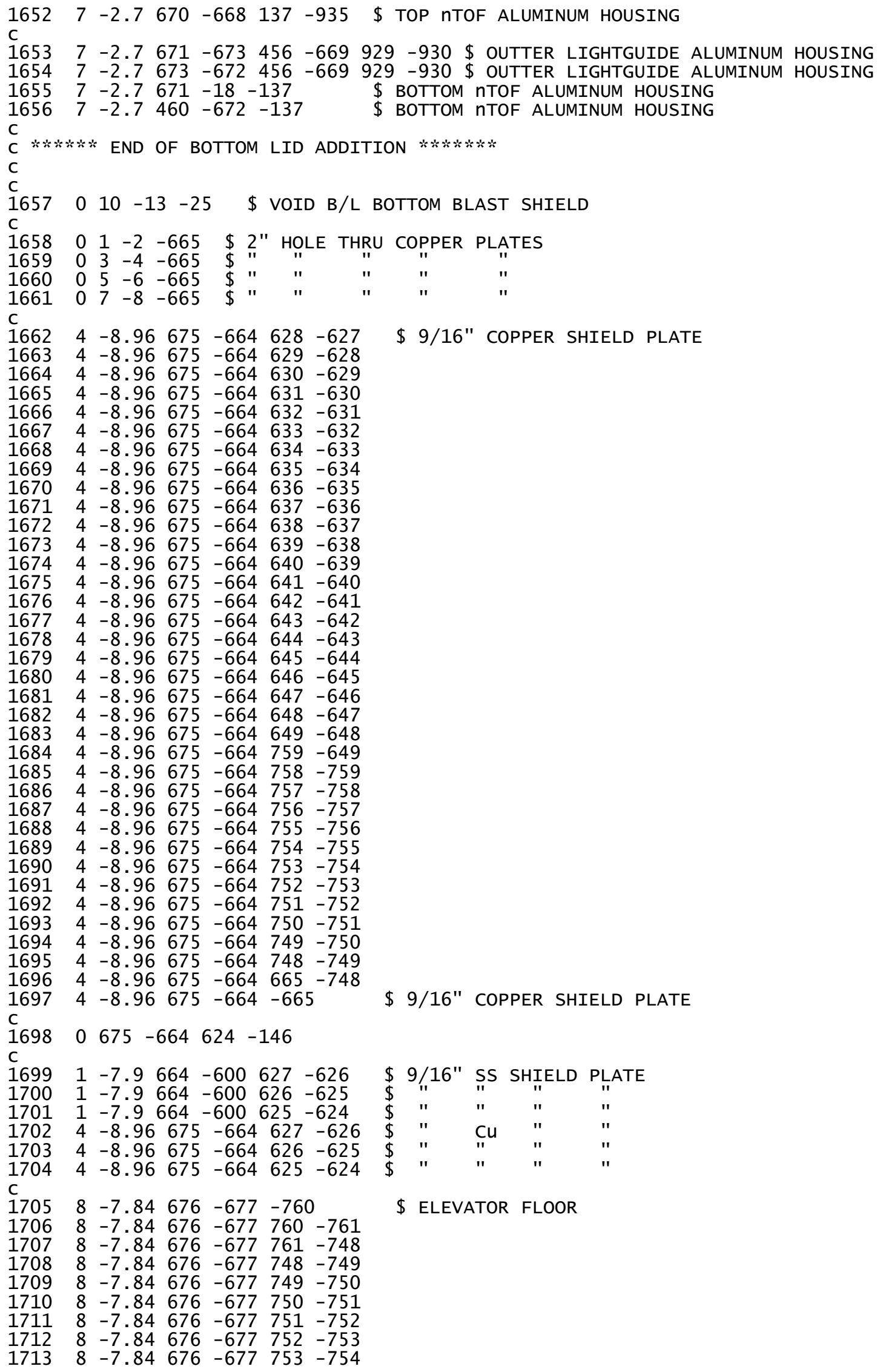




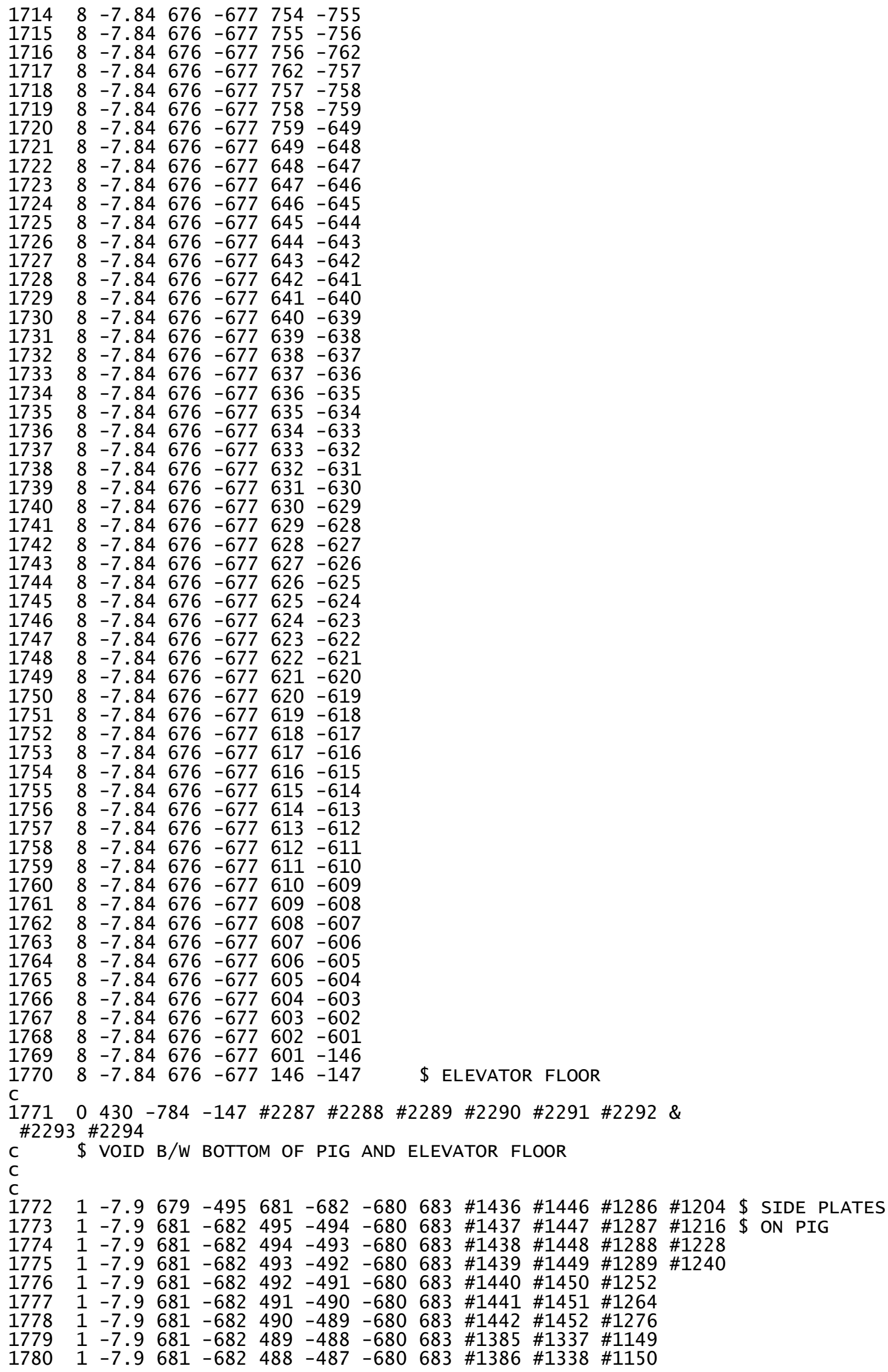


$\begin{array}{lllllllllll}1781 & 1 & -7.9 & 681 & -682 & 487 & -486 & -680 & 683 & \# 1387 & \# 1339\end{array}$ \#1151

$\begin{array}{lllllllllll}1782 & 1 & -7.9 & 681 & -682 & 486 & -485 & -680 & 683 & \# 1388 & \# 1340\end{array}$ \#1152

$\begin{array}{lllllllllllll}1783 & 1 & -7.9 & 681 & -682 & 485 & -484 & -680 & 683 & \# 1389 & \# 1341 & \text { \#1153 }\end{array}$

$\begin{array}{lllllllllll}1784 & 1 & -7.9 & 681 & -682 & 484 & -483 & -680 & 683 & \# 1390 & \# 1342\end{array}$ \#1154

$\begin{array}{llllllllllll}1785 & 1 & -7.9 & 681 & -682 & 483 & -482 & -680 & 683 & \# 1391 & \# 1343 & \# 1155\end{array}$

$\begin{array}{llllllllllll}1786 & 1 & -7.9 & 681 & -682 & 482 & -471 & -680 & 683 & \# 1392 & \# 1344 & \text { \#1156 }\end{array}$

$\begin{array}{lllllllllllll}1787 & 1 & -7.9 & 681 & -682 & 471 & -472 & -680 & 683 & \# 1402 & \# 1354 & \# 1129\end{array}$

$\begin{array}{lllllllllll}1788 & 1 & -7.9 & 681 & -682 & 472 & -473 & -680 & 683 & \# 1403 & \text { \#1355 }\end{array}$

$\begin{array}{llllllllllll}1789 & 1 & -7.9 & 681 & -682 & 473 & -474 & -680 & 683 & \# 1404 & \# 1356 & \text { \#1131 }\end{array}$

$\begin{array}{lllllllllll}1790 & 1 & -7.9 & 681 & -682 & 474 & -475 & -680 & 683 & \# 1405 & \# 1357\end{array}$ \#1132

$\begin{array}{lllllllllll}1791 & 1 & -7.9 & 681 & -682 & 475 & -476 & -680 & 683 & \# 1406 & \# 1358\end{array}$ \#1133

$\begin{array}{lllllllllllll}1792 & 1 & -7.9 & 681 & -682 & 476 & -477 & -680 & 683 & \# 1407 & \# 1359 & \text { \#1134 }\end{array}$

$\begin{array}{lllllllllll}1793 & 1 & -7.9 & 681 & -682 & 477 & -478 & -680 & 683 & \# 1408 & \# 1360\end{array}$ \#1135

$\begin{array}{lllllllllllll}1794 & 1 & -7.9 & 681 & -682 & 478 & -479 & -680 & 683 & \# 1409 & \# 1361 & \# 1136\end{array}$

$\begin{array}{lllllllllll}1795 & 1 & -7.9 & 681 & -682 & 479 & -480 & -680 & 683 & \# 1410 & \# 1362\end{array}$ \#1137

$\begin{array}{lllllllllllll}1796 & 1 & -7.9 & 681 & -682 & 480 & -481 & -680 & 683 & \# 1411 & \# 1363 & \# 1138\end{array}$

$\begin{array}{llllllllllll}1797 & 1 & -7.9 & 681 & -682 & 481 & -434 & -680 & 683 & \# 1412 & \# 1364 & \text { \#1139 }\end{array}$

$\begin{array}{lllllllllll}1798 & 1 & -7.9 & 681 & -682 & 434 & -435 & -680 & 683 & \# 1413 & \# 1365\end{array}$ \#1063

$\begin{array}{lllllllllllll}1799 & 1 & -7.9 & 681 & -682 & 435 & -436 & -680 & 683 & \text { \#1414 } & \text { \#1366 } & \text { \#1064 }\end{array}$

$\begin{array}{llllllllllll}1800 & 1 & -7.9 & 681 & -682 & 436 & -437 & -680 & 683 & \# 1415 & \text { \#1367 } & \text { \#1065 }\end{array}$

$\begin{array}{llllllllllll}1801 & 1 & -7.9 & 681 & -682 & 437 & -438 & -680 & 683 & \# 1416 & \# 1368 & \text { \#1066 }\end{array}$

$\begin{array}{lllllllllll}1802 & 1 & -7.9 & 681 & -682 & 438 & -439 & -680 & 683 & \# 1417 & \# 1369\end{array}$ \#1067

$\begin{array}{llllllllllll}1803 & 1 & -7.9 & 681 & -682 & 439 & -440 & -680 & 683 & \# 1418 & \text { \#1370 \#1068 }\end{array}$

$\begin{array}{llllllllllll}1804 & 1 & -7.9 & 681 & -682 & 440 & -441 & -680 & 683 & \# 1419 & \text { \#1371 \#1069 }\end{array}$

$\begin{array}{llllllllllll}1805 & 1 & -7.9 & 681 & -682 & 441 & -442 & -680 & 683 & \# 1420 & \# 1372 & \text { \#1070 }\end{array}$

$\begin{array}{lllllllllll}1806 & 1 & -7.9 & 681 & -682 & 442 & -443 & -680 & 683 & \# 1421 & \# 1373\end{array}$ \#1071

$\begin{array}{lllllllllll}1807 & 1 & -7.9 & 681 & -682 & 443 & -444 & -680 & 683 & \# 1422 & \# 1374\end{array}$ \#1072

$\begin{array}{lllllllllll}1808 & 1 & -7.9 & 681 & -682 & 444 & -445 & -680 & 683 & \# 1423 & \# 1375\end{array}$ \#1073

$\begin{array}{lllllllllll}1809 & 1 & -7.9 & 681 & -682 & 445 & -18 & -680 & 683 & \# 1424 & \# 1376\end{array}$ \#1074

$\begin{array}{lllllllllll}1810 & 1 & -7.9 & 681 & -682 & 18 & -460 & -680 & 683 & \# 1425 & \# 1377\end{array}$ \#1075

$\begin{array}{llllllllll}1811 & 1 & -7.9 & 681 & -682 & 460 & -446 & -680 & 683 & \# 1426\end{array}$ \#1378 \#1076

$\begin{array}{llllllllllll}1812 & 1 & -7.9 & 681 & -682 & 446 & -447 & -680 & 683 & \# 1427 & \# 1379 & \text { \#1077 }\end{array}$

$\begin{array}{llllllllllll}1813 & 1 & -7.9 & 681 & -682 & 447 & -448 & -680 & 683 & \# 1428 & \# 1380 & \# 1078\end{array}$

$\begin{array}{lllllllllll}1814 & 1 & -7.9 & 681 & -682 & 448 & -449 & -680 & 683 & \# 1429 & \# 1381\end{array}$ \#1079

$\begin{array}{llllllllllll}1815 & 1 & -7.9 & 681 & -682 & 449 & -428 & -680 & 683 & \# 1430 & \# 1382 & \# 1080\end{array}$

$\begin{array}{lllllllllll}1816 & 1 & -7.9 & 681 & -682 & 428 & -429 & -680 & 683 & \# 1431 & \# 1383\end{array}$ \#1081

$\begin{array}{llllllllllll}1817 & 1 & -7.9 & 681 & -682 & 429 & -430 & -680 & 683 & \# 1432 & \# 1384 & \text { \#1082 }\end{array}$

$\begin{array}{llllll}1818 & 0 & 498 & -679 & 509 & -147\end{array}$

$\begin{array}{lllllllll}1819 & 0 & 679 & -430 & -680 & 683 & -681 & -147 & \text { \$ VOIDS AROUND PIG }\end{array}$

$\begin{array}{llllllll}1820 & 0 & 679 & -430 & -680 & 683 & 682 & -147\end{array}$

$\begin{array}{lllllllll}1821 & 0 & 679 & -430 & -685 & 684 & -681 & -147 & \# 2289\end{array}$

C

$\begin{array}{lllllllll}1822 & 0 & 679 & -430 & -685 & 684 & 682 & -147 & \# 2291\end{array}$

C

$\begin{array}{llllllll}1823 & 0 & 679 & -430 & -683 & 685 & 509 & -147\end{array}$

C $\begin{array}{llllllll}1823 & 0 & 679 & -430 & -683 & 685 & 509 & -147\end{array}$

C $\begin{array}{llllllll}1824 & 0 & 679 & -430 & 681 & -682 & 684 & 509\end{array}$

$\begin{array}{llllll}1824 & 0 & 679 & -471 & -684 & -147\end{array}$

$\begin{array}{llllll}1825 & 0 & 471 & -434 & -684 & -147\end{array}$

$\begin{array}{lllllllllll}1826 & 0 & 434 & -430 & -684 & -21 & \text { \#2287 \#2289 \#2291 }\end{array}$

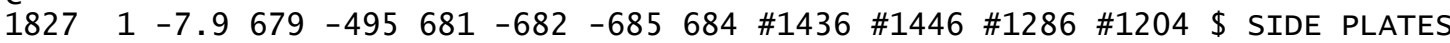
ON PIG

$\begin{array}{llllllllllll}1828 & 1 & -7.9 & 681 & -682 & 495 & -494 & -685 & 684 & \# 1437 & \# 1447 & \# 1287\end{array}$ \#1216

$\begin{array}{llllllllllll}1829 & 1 & -7.9 & 681 & -682 & 494 & -493 & -685 & 684 & \# 1438 & \# 1448 & \# 1288\end{array}$ \#1228

$\begin{array}{llllllllllll}1830 & 1 & -7.9 & 681 & -682 & 493 & -492 & -685 & 684 & \# 1439 & \# 1449 & \text { \#1289 \#1240 }\end{array}$

$\begin{array}{llllllllllll}1831 & 1 & -7.9 & 681 & -682 & 492 & -491 & -685 & 684 & \# 1440 & \# 1450 & \# 1252\end{array}$

$\begin{array}{llllllllllll}1832 & 1 & -7.9 & 681 & -682 & 491 & -490 & -685 & 684 & \# 1441 & \# 1451 & \# 1264\end{array}$

$\begin{array}{llllllllll}1833 & 1 & -7.9 & 681 & -682 & 490 & -489 & -685 & 684 & \# 1442\end{array}$ \#1452 \#1276

$\begin{array}{llllllllllll}1834 & 1 & -7.9 & 681 & -682 & 489 & -488 & -685 & 684 & \# 1385 & \# 1337 & \# 1149\end{array}$

$\begin{array}{llllllllllll}1835 & 1 & -7.9 & 681 & -682 & 488 & -487 & -685 & 684 & \# 1386 & \# 1338 & \text { \#1150 }\end{array}$

$\begin{array}{llllllllllll}1836 & 1 & -7.9 & 681 & -682 & 487 & -486 & -685 & 684 & \# 1387 & \# 1339 & \# 1151\end{array}$

$\begin{array}{lllllllllll}1837 & 1 & -7.9 & 681 & -682 & 486 & -485 & -685 & 684 & \# 1388 & \# 1340\end{array}$ \#1152

$\begin{array}{llllllllllll}1838 & 1 & -7.9 & 681 & -682 & 485 & -484 & -685 & 684 & \# 1389 & \# 1341 & \# 1153\end{array}$

$\begin{array}{lllllllllll}1839 & 1 & -7.9 & 681 & -682 & 484 & -483 & -685 & 684 & \# 1390 & \# 1342\end{array}$ \#1154 


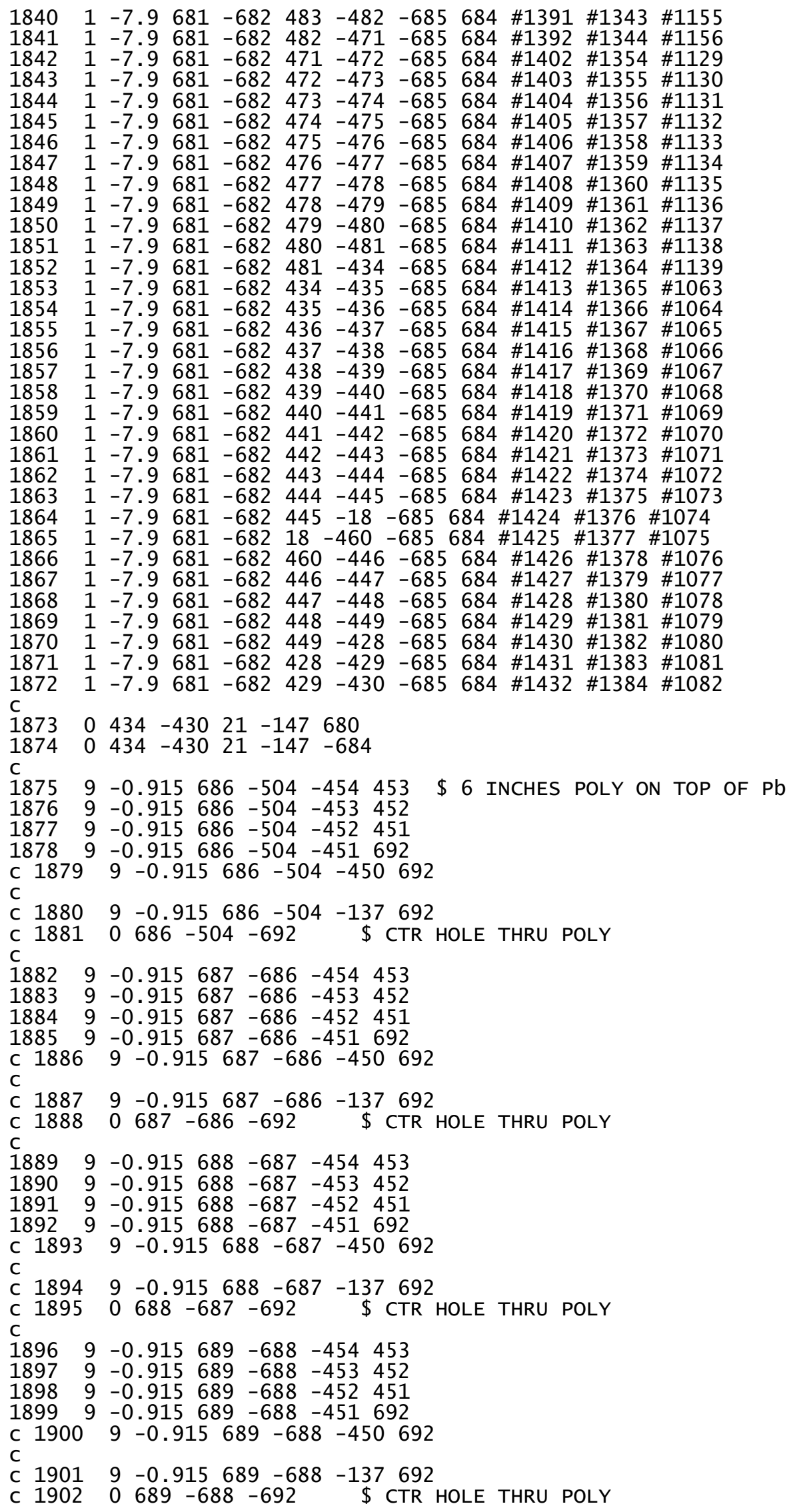




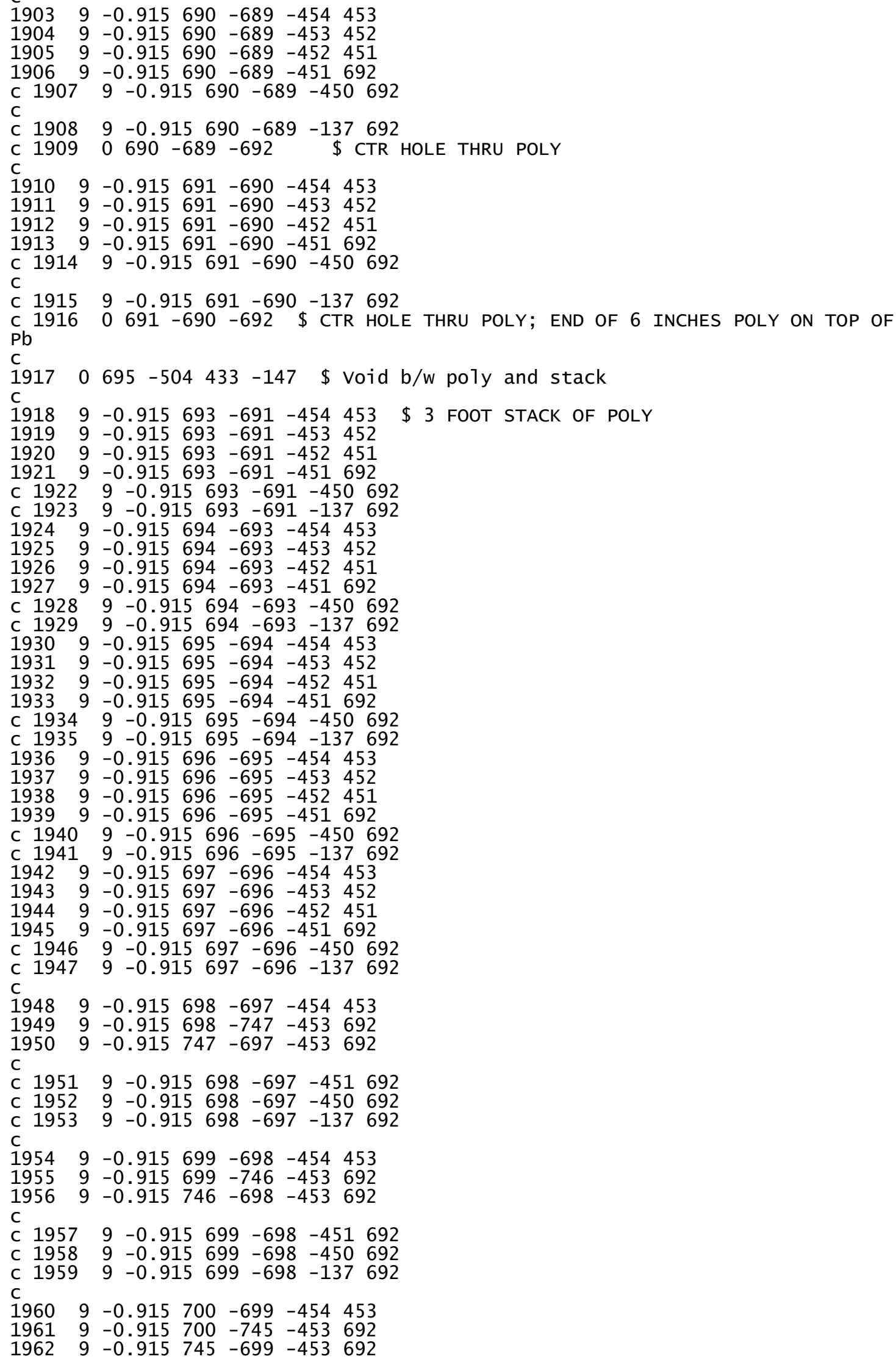




$$
\begin{aligned}
& \text { C } \\
& \begin{array}{lllllllll}
\text { C } & 1963 & 9 & -0.915 & 700 & -699 & -451 & 692
\end{array}
\end{aligned}
$$

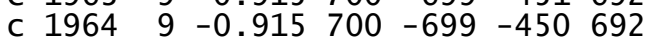

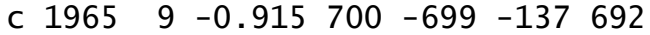

$$
\begin{aligned}
& \text { C } \\
& \begin{array}{llllllll}
1966 & 9 & -0.915 & 701 & -700 & -454 & 453
\end{array} \\
& \begin{array}{lllllll}
1967 & 9 & -0.915 & 701 & -744 & -453 & 692
\end{array} \\
& \begin{array}{lllllll}
1968 & 9 & -0.915 & 744 & -700 & -453 & 692
\end{array}
\end{aligned}
$$




$$
\begin{aligned}
& \text { C } \\
& \begin{array}{lllllllll}
\text { C } & 2017 & 9 & -0.915 & 709 & -708 & -451 & 692
\end{array}
\end{aligned}
$$

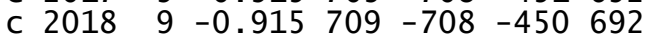

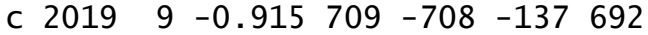

$$
\begin{aligned}
& \mathrm{C} \\
& \begin{array}{llllllll}
2020 & 9 & -0.915 & 710 & -709 & -454 & 453
\end{array} \\
& \begin{array}{lllllll}
2021 & 9 & -0.915 & 710 & -723 & -453 & 692
\end{array}
\end{aligned}
$$

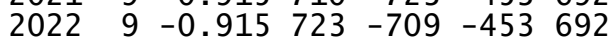

$$
\begin{aligned}
& \begin{array}{llllllll}
\text { C } & 2023 & 9 & -0.915 & 710 & -709 & -451 & 692
\end{array} \\
& \begin{array}{lllllll}
\text { C } 2024 & 9 & -0.915 & 710 & -709 & -450 & 692
\end{array}
\end{aligned}
$$

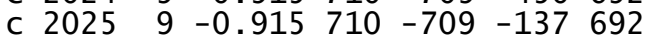




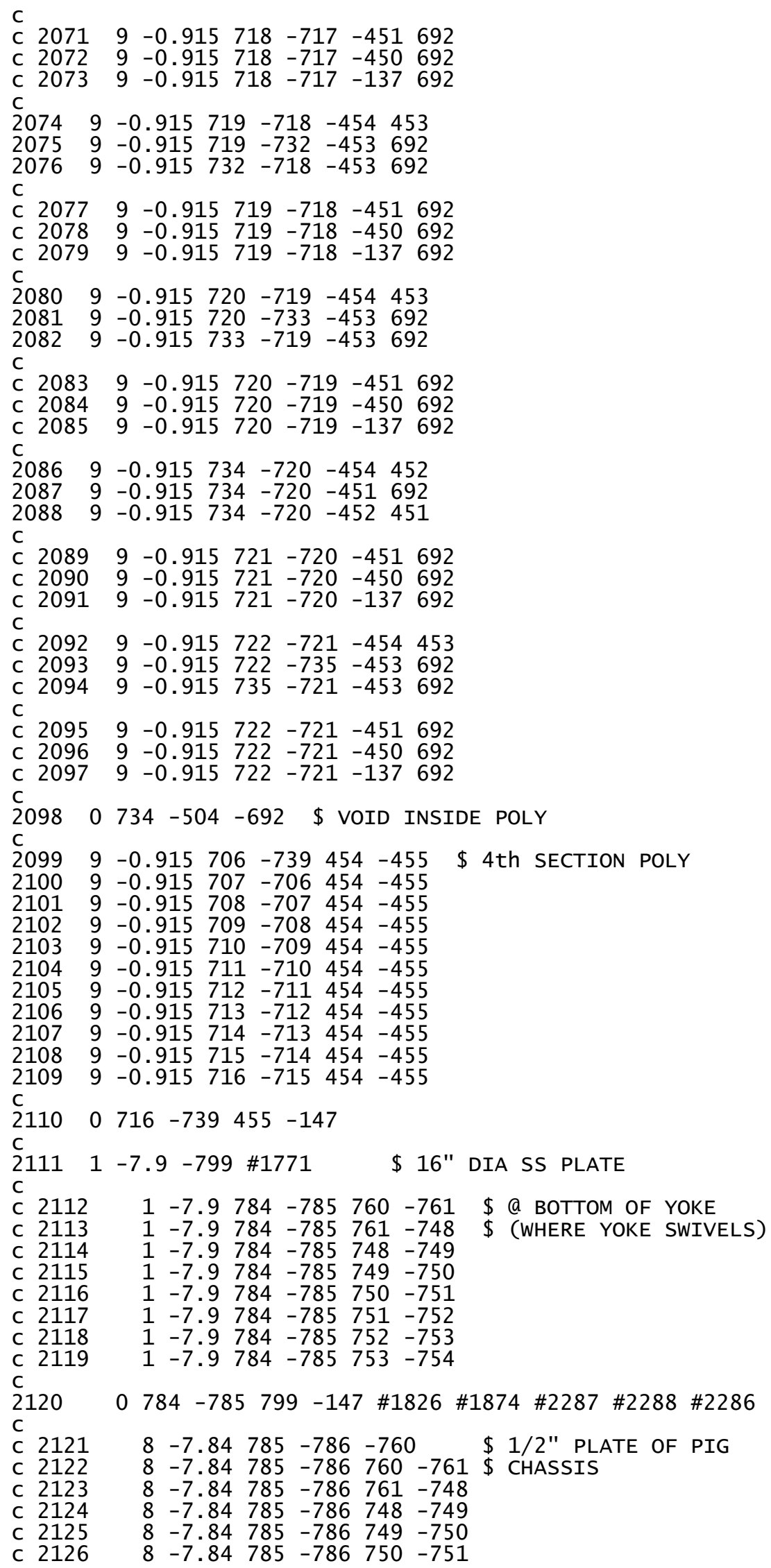




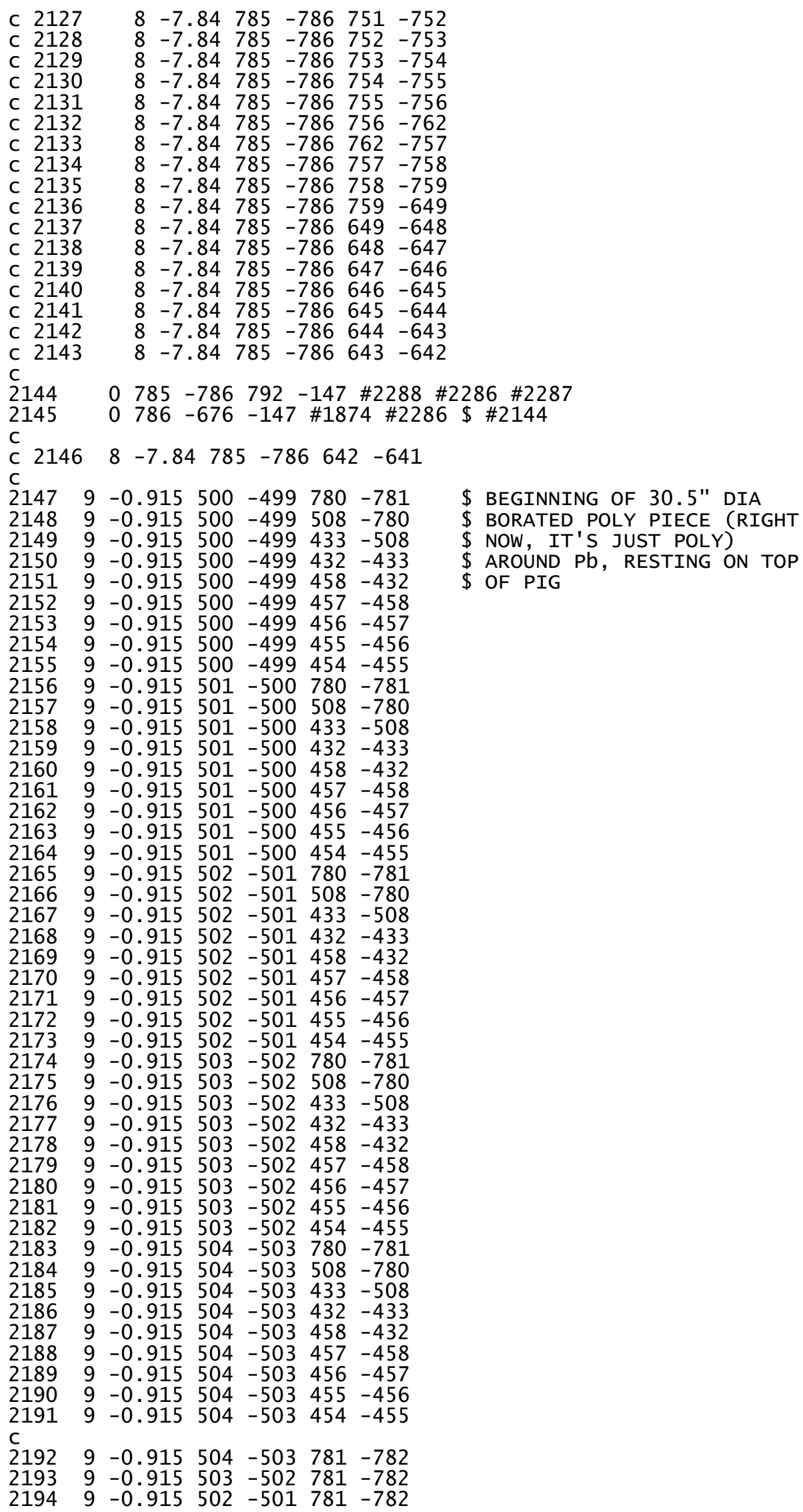




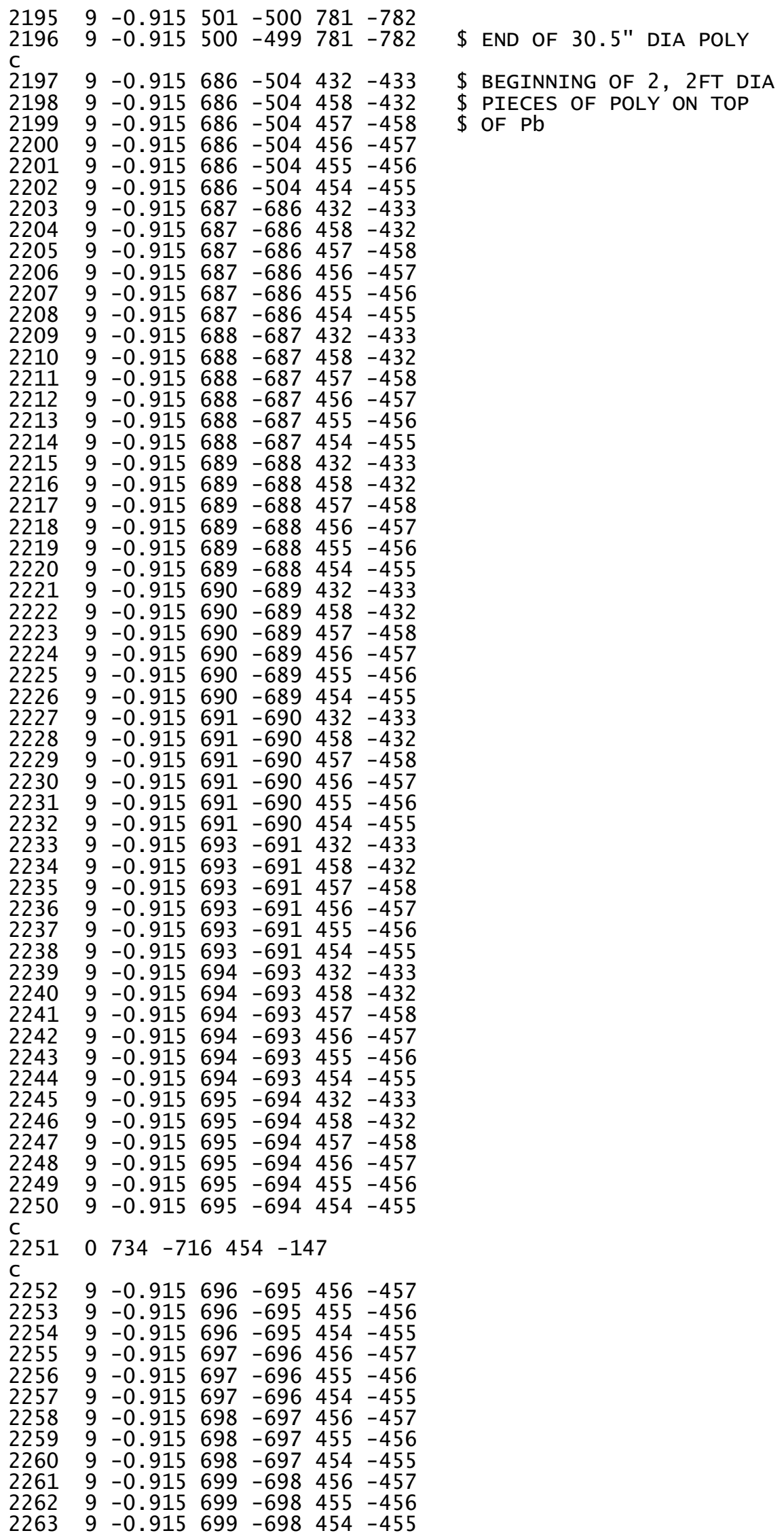




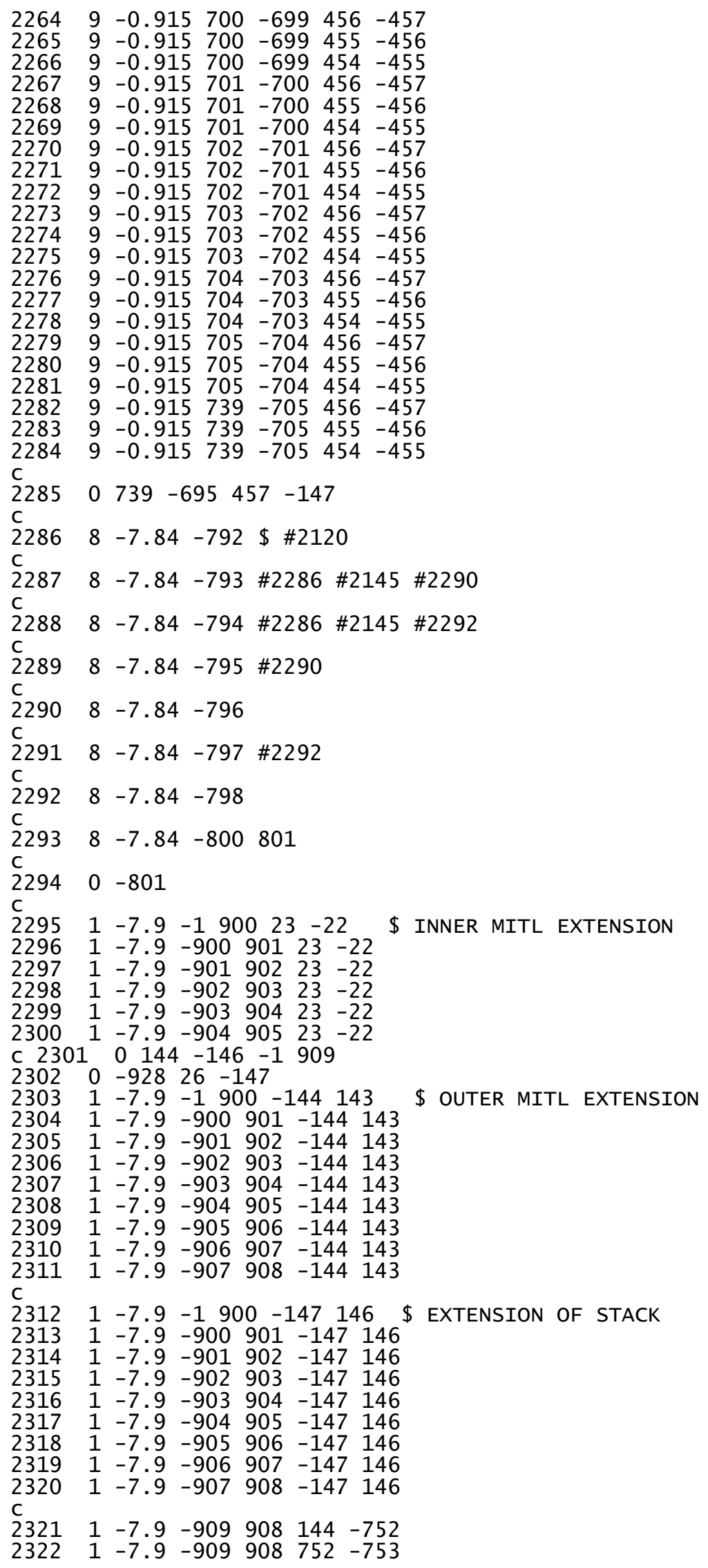




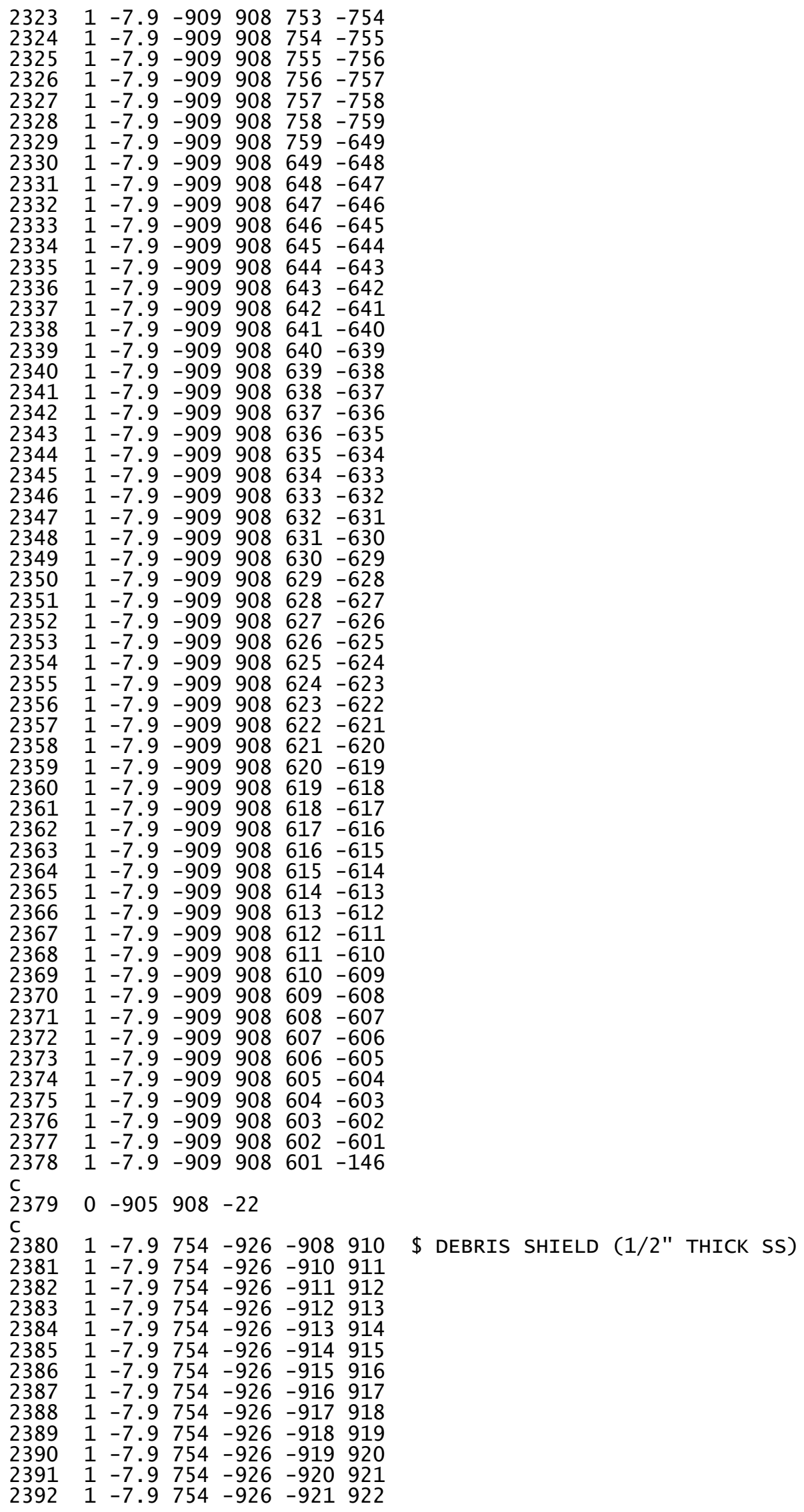




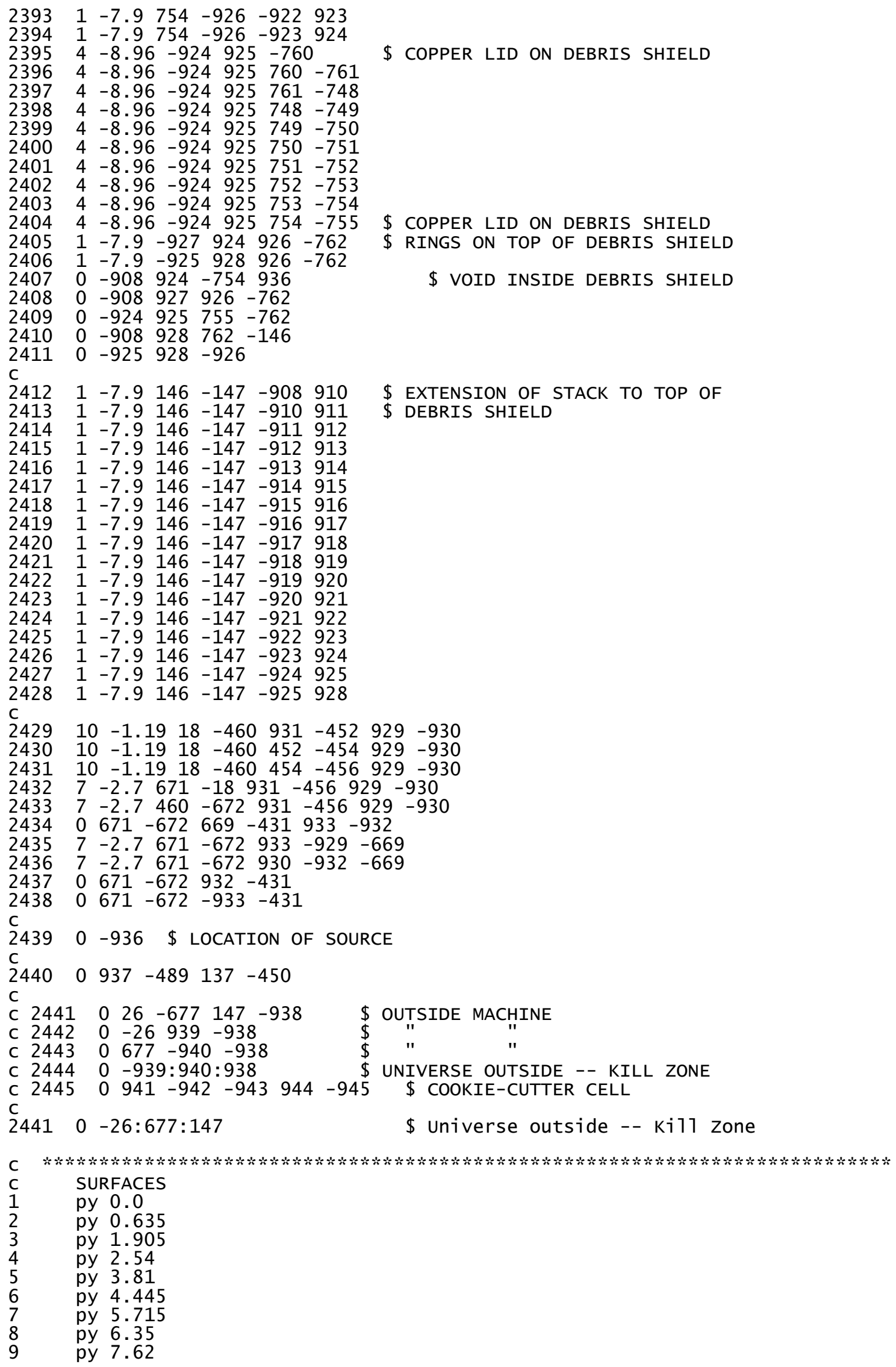




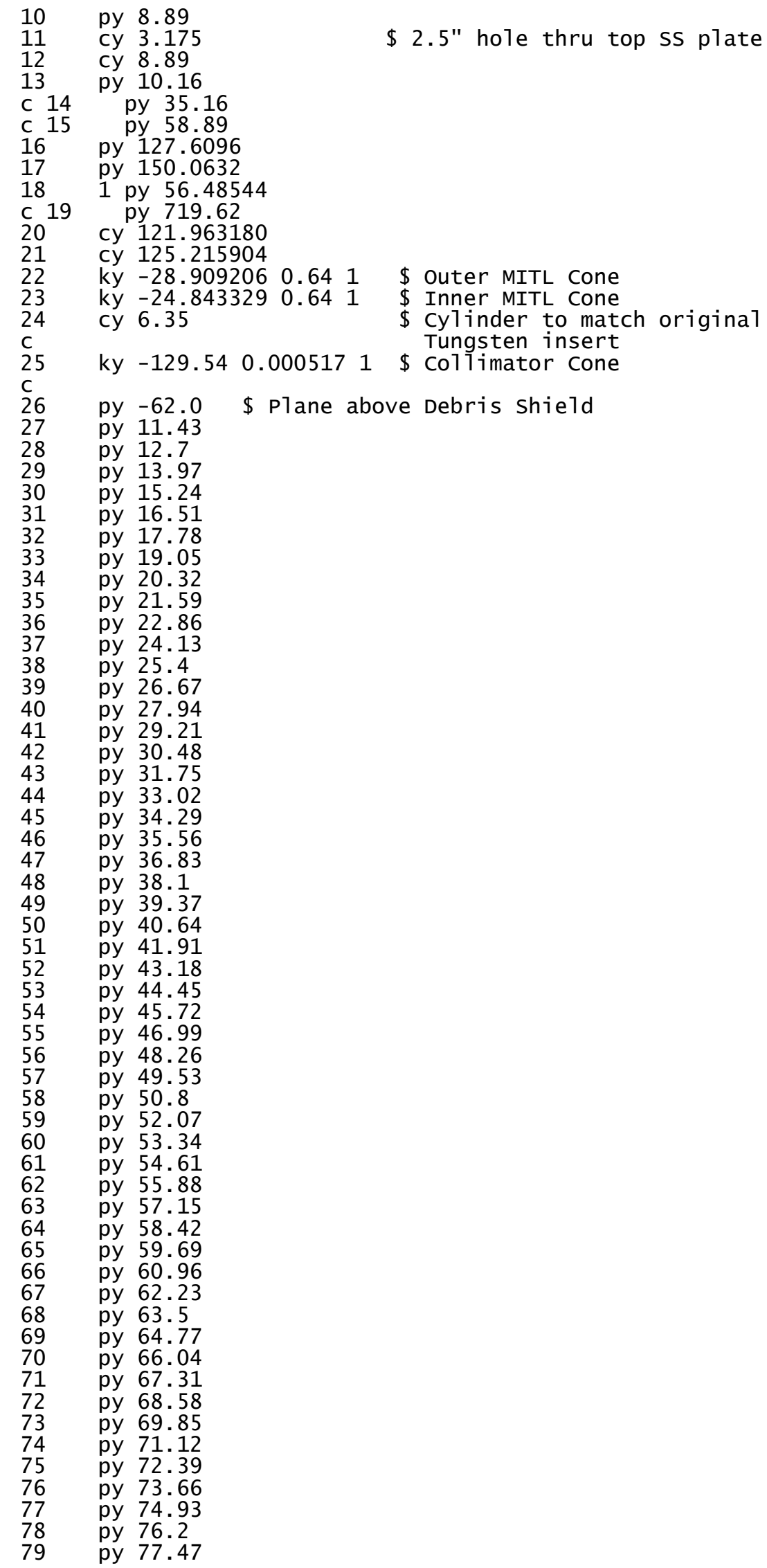




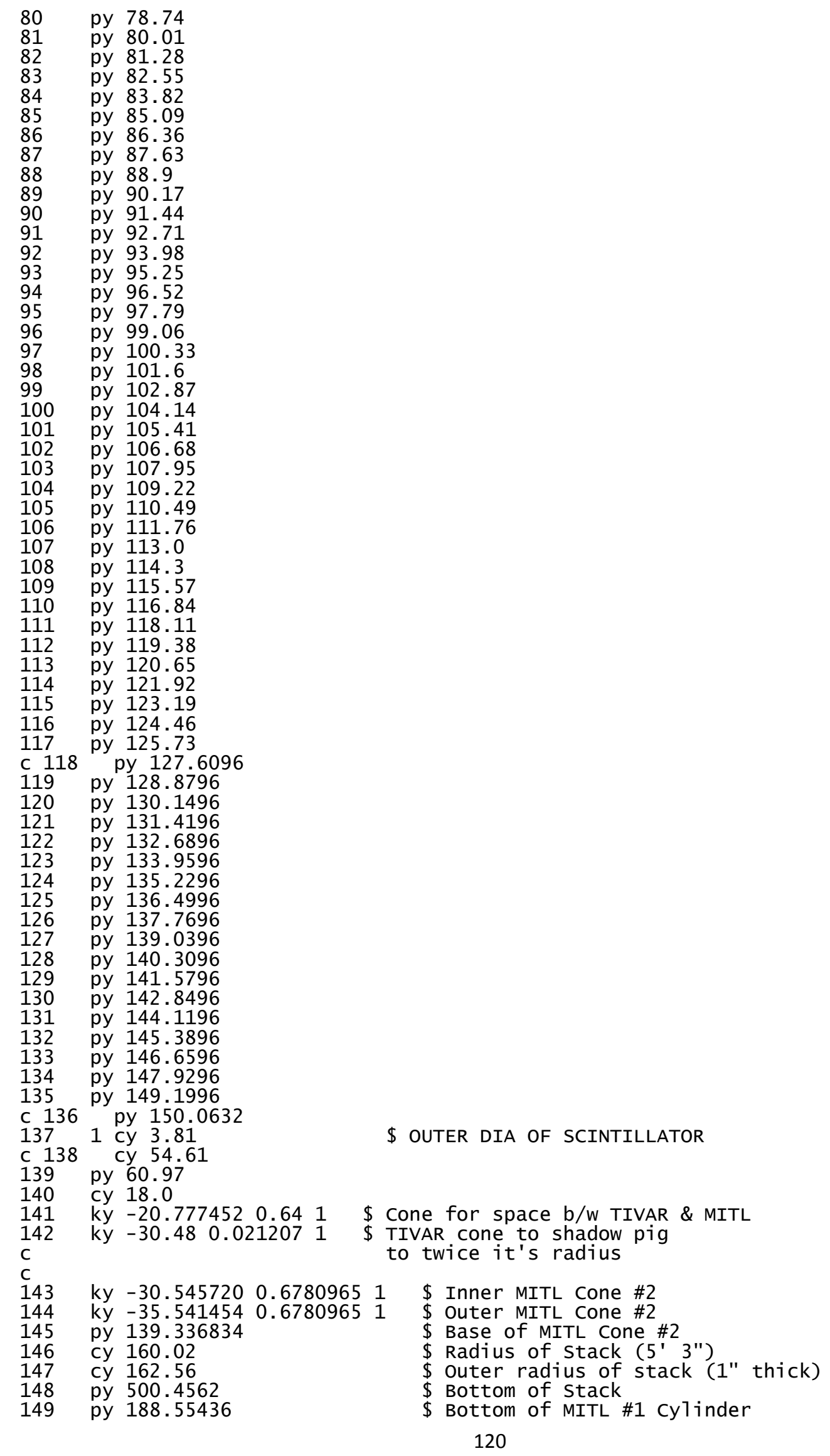


c

150 py 151.3332

151 py 152.6032

152 py 153.8732

153 py 155.1432

154 py 156.4132

155 py 157.6832

156 py 158.9532

157 py 160.2232

158 py 161.4932

159 py 162.7632

160 py 164.0332

161 py 165.3032

162 py 166.5732

163 py 167.8432

164 py 169.1132

165 py 170.3832

166 py 171.6532

167 py 172.9232

168 py 174.1332

169 py 175.4632

170 py 176.7332

171 py 178.0032

172 py 179.2732

173 py 180.5432

174 py 181.8132

175 py 183.0832

176 py 184.3532

177 py 185.6232

178 py 186.8932

179 py 188.1632

180 py 190.0

181 py 191.25

182 py 192.5

183 py 193.75

184 py 195.0

185 py 196.25

186 py 197.5

187 py 198.75

188 py 200.0

189 py 201.25

190 py 202.5

191 py 203.75

192 py 205.0

193 py 206.25

194 py 207.5

195 py 208.75

196 py 210.0

197 py 211.25

198 py 212.5

199 py 213.75

200 py 215.0

201 py 216.25

202 py 217.5

203 py 218.75

204 py 220.0

205 py 221.25

206 py 222.5

207 py 223.75

208 py 225.0

209 py 226.25

210 py 227.5

211 py 228.75

212 py 230.0

213 py 231.25

214 py 232.5

215 py 233.75

216 py 235.0

217 py 236.25

218 py 237.5 
219

220

221

222

224

225

226

227

228

229

230

231

232

233

234

235

236

237

238

239

241

242

243

244

245

246
247

248

249

250

251

252

253

254

255

256

257
258

259

260

261

262

263

264

265

266

267

268

269

270

271

273

274

275

276

277
278

278

279
280

281

282

283

284

285

286

287

288

289

py 238.75

py 240.0

py 241.25

py 242.5

py 243.75

py 245.0

py 246.25

py 247.5

py 248.75

py 250.0

py 251.25

py 252.5

py 253.75

py 255.0

py 256.25

py 257.5

py 258.75

py 260.0

py 261.25

py 262.5

py 263.75

py 265.0

py 266.25

py 267.5

py 268.75

py 270.0

py 271.25

py 272.5

py 273.75

py 275.0

py 276.25

py 277.5

py 278.75

py 280.0

py 281.25

py 282.5

py 283.75

py 285.0

py 286.25

py 287.5

py 288.75

py 290.0

py 291.25

py 292.5

py 293.75

py 295.0

py 296.25

py 297.5

py 298.75

py 300.0

py 301.25

py 302.5

py 303.75

py 305.0

py 306.25

py 307.5

py 308.75

py 310.0

py 311.25

py 312.5

py 313.75

py 315.0

py 316.25

py 317.5

py 318.75

py 320.0

py 321.25

py 322.5

py 323.75

py 325.0

py 326.25

py 327.5 


$\begin{array}{ll}291 & \text { py } 328.75 \\ 292 & \text { py } 330.0 \\ 293 & \text { py } 331.25 \\ 294 & \text { py } 332.5 \\ 295 & \text { py } 333.75 \\ 296 & \text { py } 335.0 \\ 297 & \text { py } 336.25 \\ 298 & \text { py } 337.5 \\ 299 & \text { py } 338.75 \\ 300 & \text { py } 340.0 \\ 301 & \text { py } 341.25 \\ 302 & \text { py } 342.5 \\ 303 & \text { py } 343.75 \\ 304 & \text { py } 345.0 \\ 305 & \text { py } 346.75 \\ 306 & \text { py } 347.5 \\ 307 & \text { py } 348.75 \\ 308 & \text { py } 350.0 \\ 309 & \text { py } 351.25 \\ 310 & \text { py } 352.5 \\ 311 & \text { py } 353.75 \\ 312 & \text { py } 355.0 \\ 313 & \text { py } 356.25 \\ 314 & \text { py } 357.5 \\ 315 & \text { py } 358.75 \\ 316 & \text { py } 360.0 \\ 317 & \text { py } 361.25 \\ 318 & \text { py } 362.5 \\ 319 & \text { py } 363.75 \\ 320 & \text { py } 365.0 \\ 321 & \text { py } 366.25 \\ 322 & \text { py } 367.5 \\ 323 & \text { py } 368.75 \\ 324 & \text { py } 370.0 \\ 325 & \text { py } 371.25 \\ 326 & \text { py } 372.5 \\ 327 & \text { py } 373.75 \\ 328 & \text { py } 375.0 \\ 329 & \text { py } 376.25 \\ 330 & \text { py } 377.5 \\ 331 & \text { py } 378.75 \\ 332 & \text { py } 380.0 \\ 333 & \text { py } 381.25 \\ 334 & \text { py } 382.5 \\ 335 & \text { py } 383.75 \\ 336 & \text { py } 385.0 \\ 337 & \text { py } 386.25 \\ 338 & \text { py } 387.5 \\ 339 & \text { py } 388.75 \\ 340 & \text { py } 390.0 \\ 341 & \text { py } 391.25 \\ 342 & \text { py } 392.5 \\ 343 & \text { py } 393.75 \\ 344 & \text { py } 395.0 \\ 345 & \text { py } 396.25 \\ 346 & \text { py } 397.5 \\ 347 & \text { py } 398.75 \\ 348 & \text { py } 400.0 \\ 349 & \text { py } 401.25 \\ 350 & \text { py } 402.5 \\ 351 & \text { py } 403.75 \\ 352 & \text { py } 405.0 \\ 353 & \text { py } 406.25 \\ 354 & \text { py } 407.5 \\ 355 & \text { py } 408.75 \\ 357 & \text { py } 410.0 \\ 359 & \text { py } 411.25 \\ 360 & \text { py } 413.5 \\ 361 & \text { py } 415.0 \\ 362 & \text { py } 417.25 \\ & \text { } 316\end{array}$




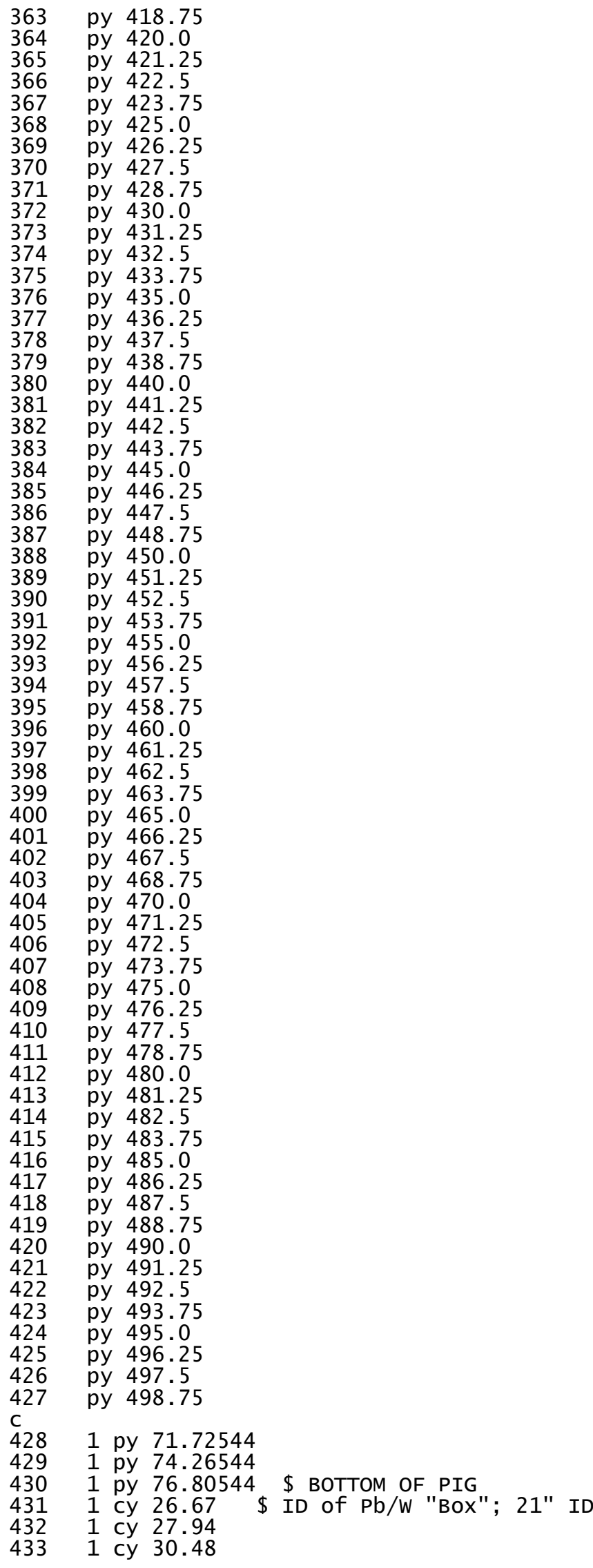


C

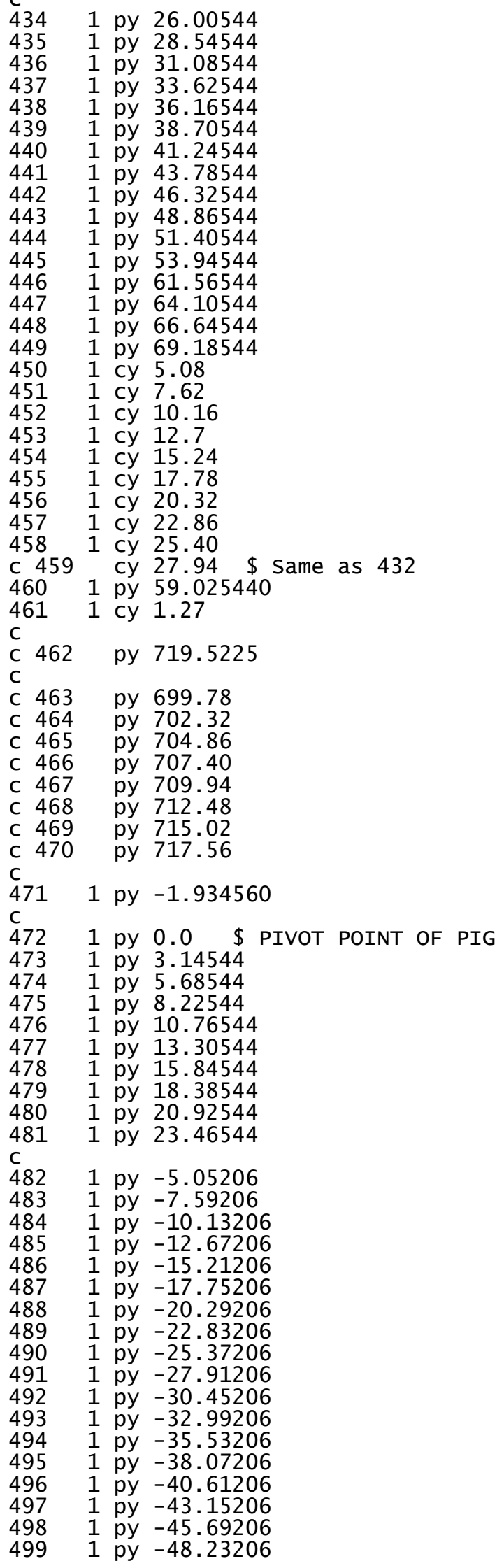




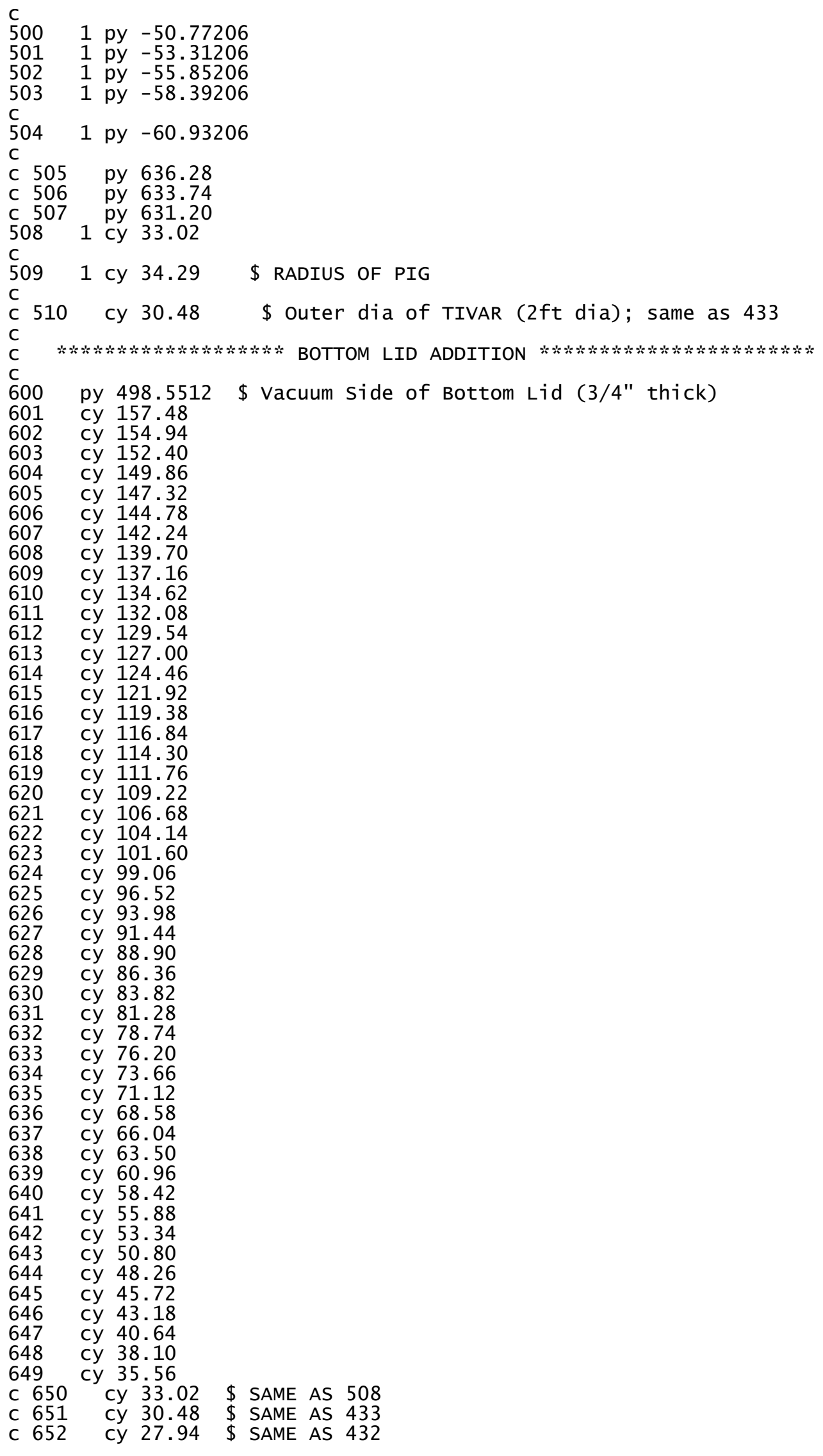




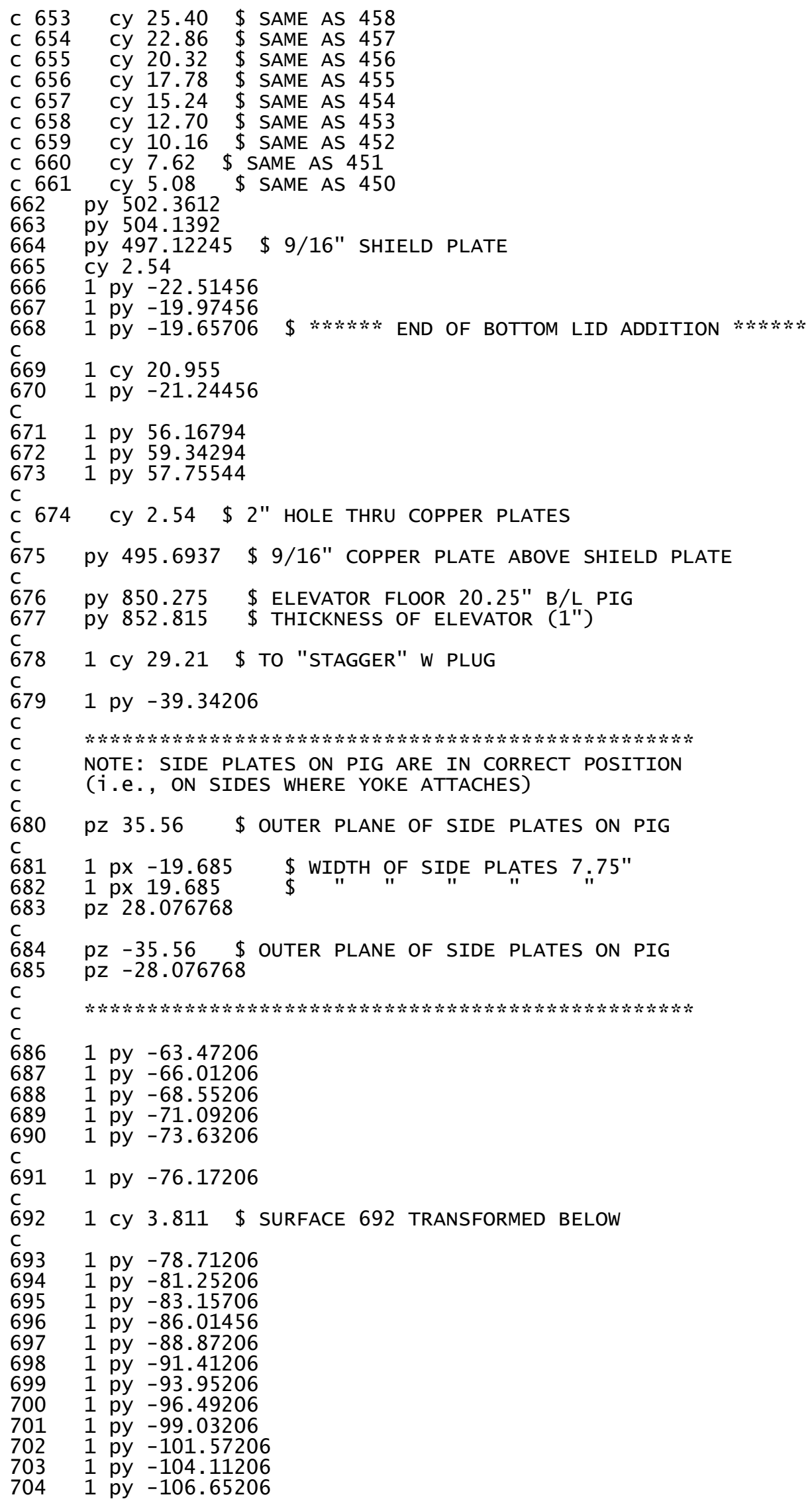




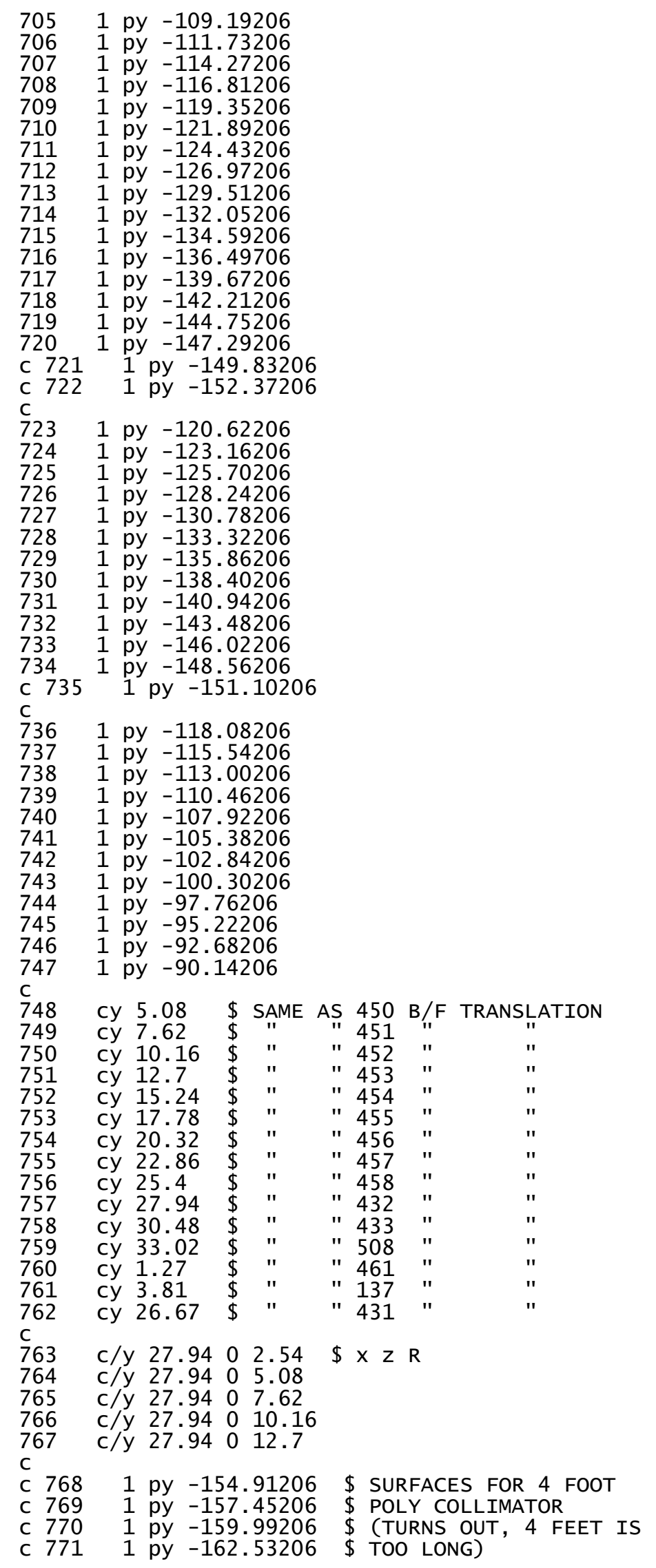




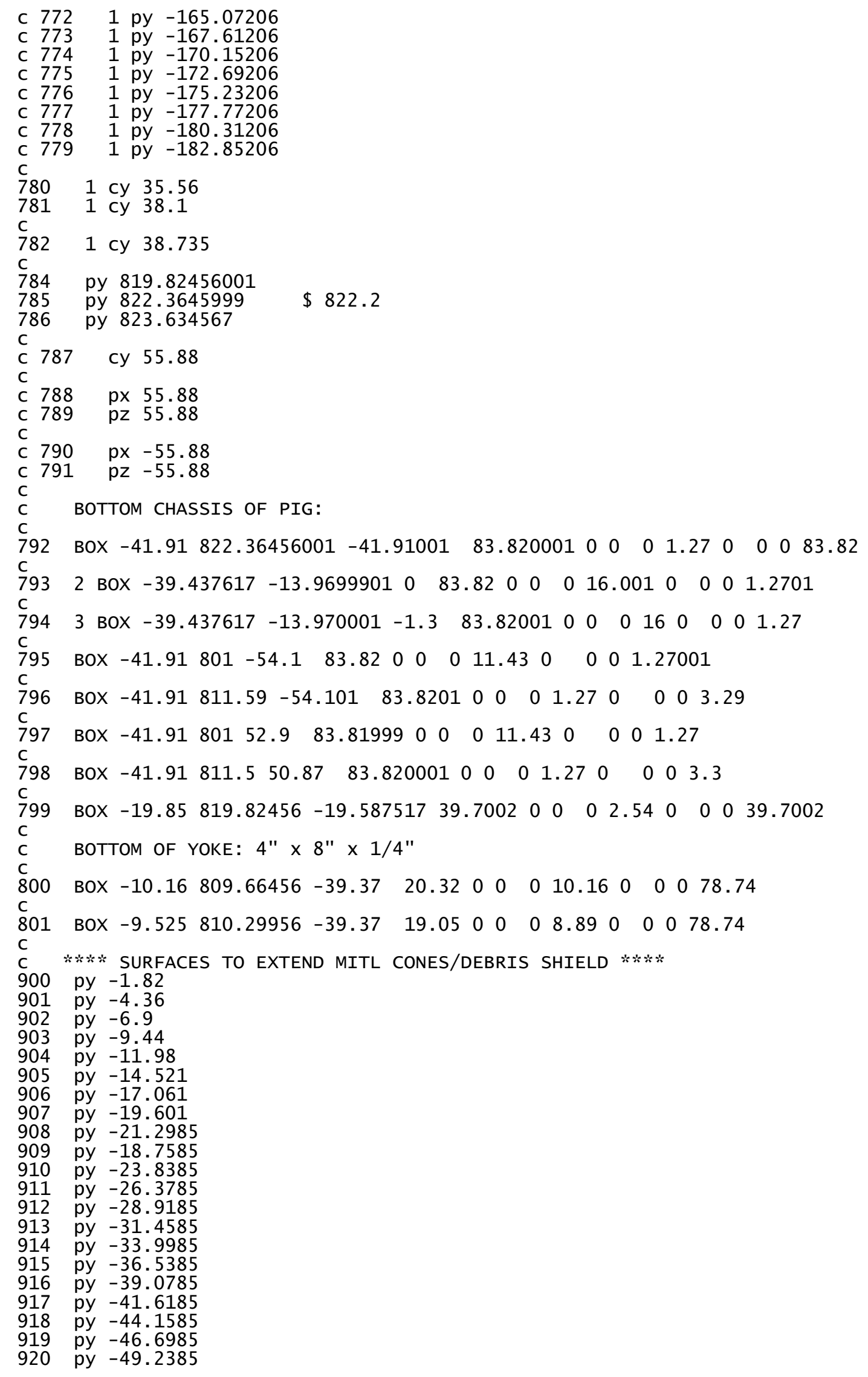




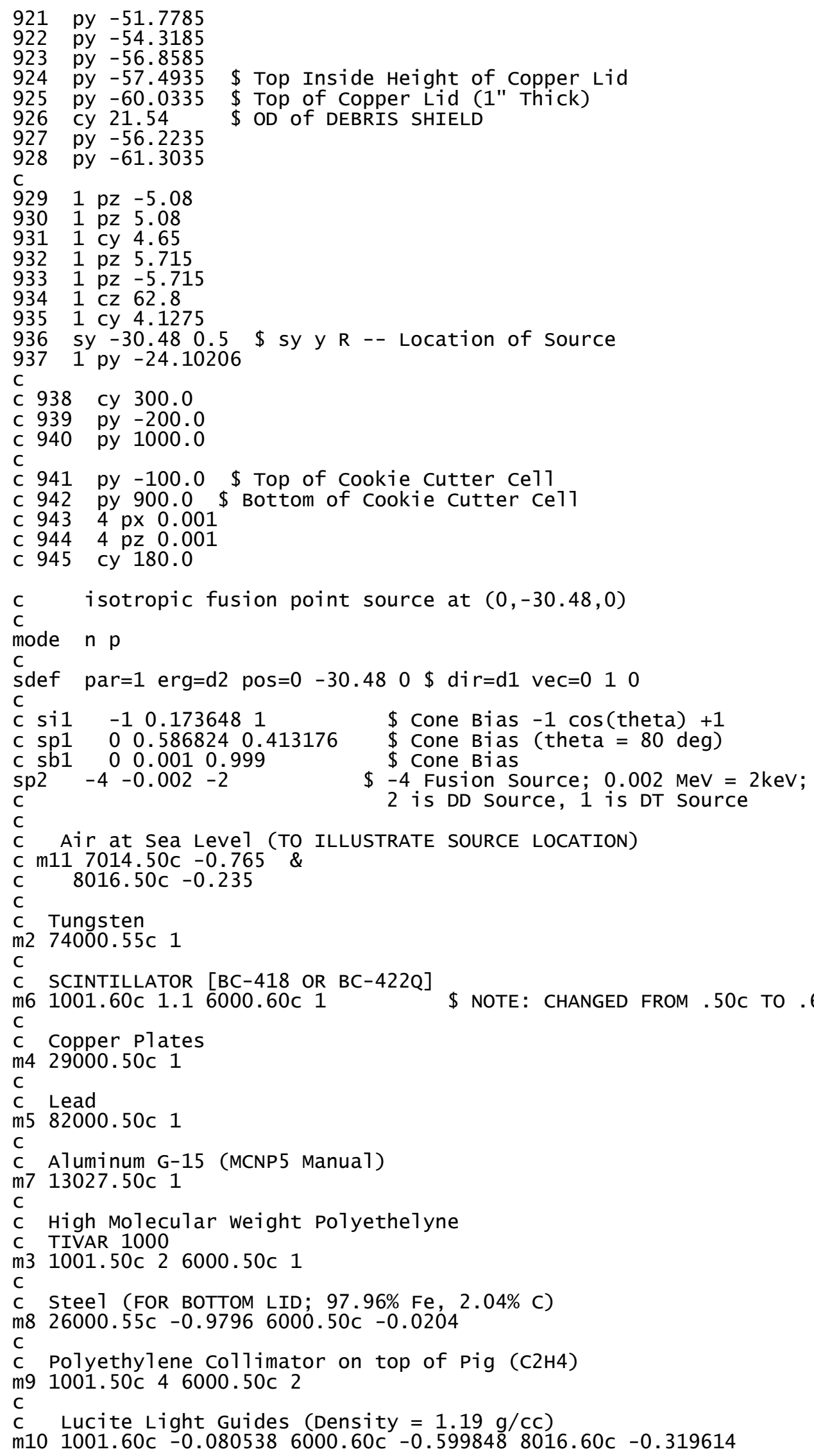




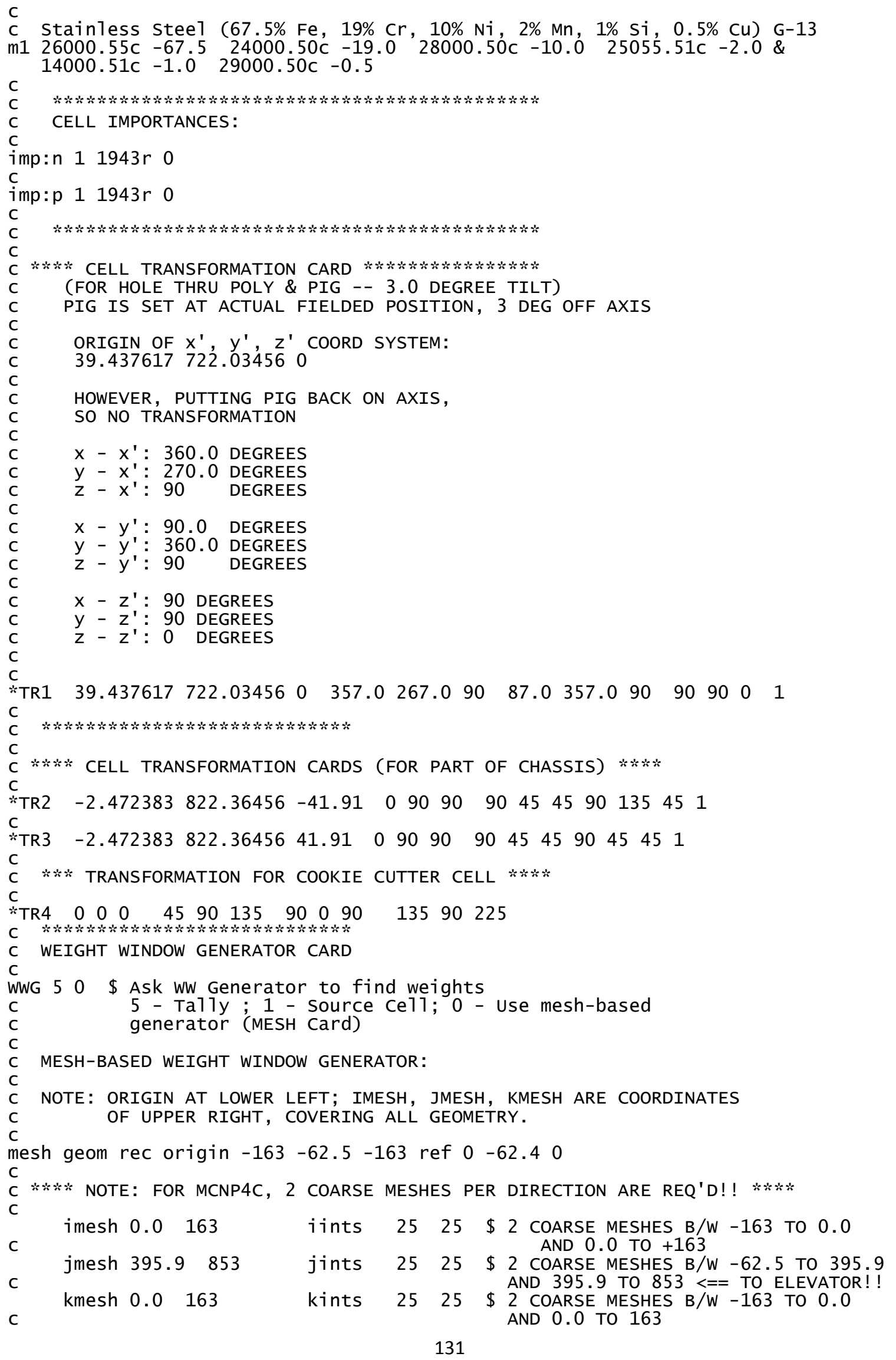




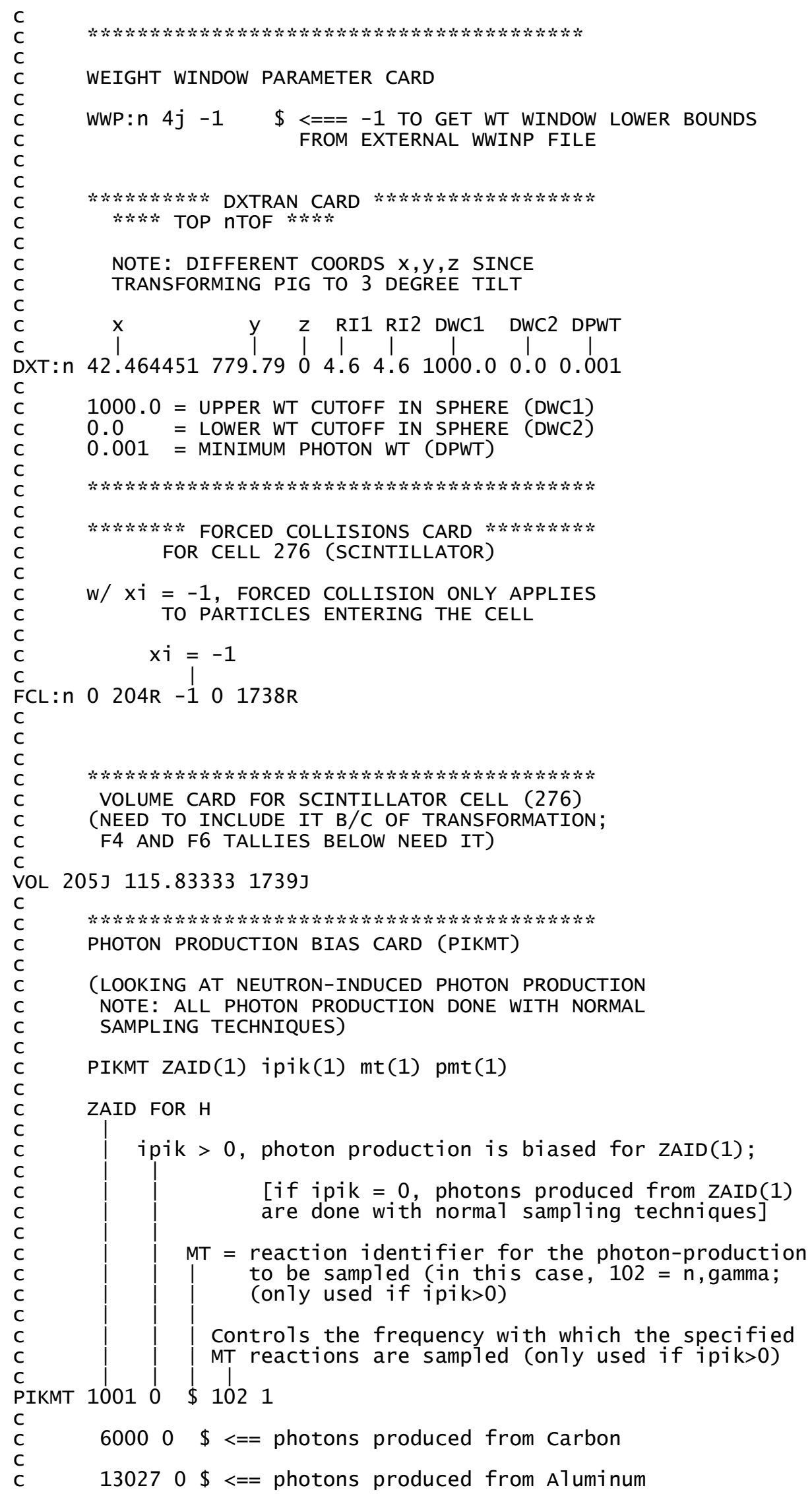




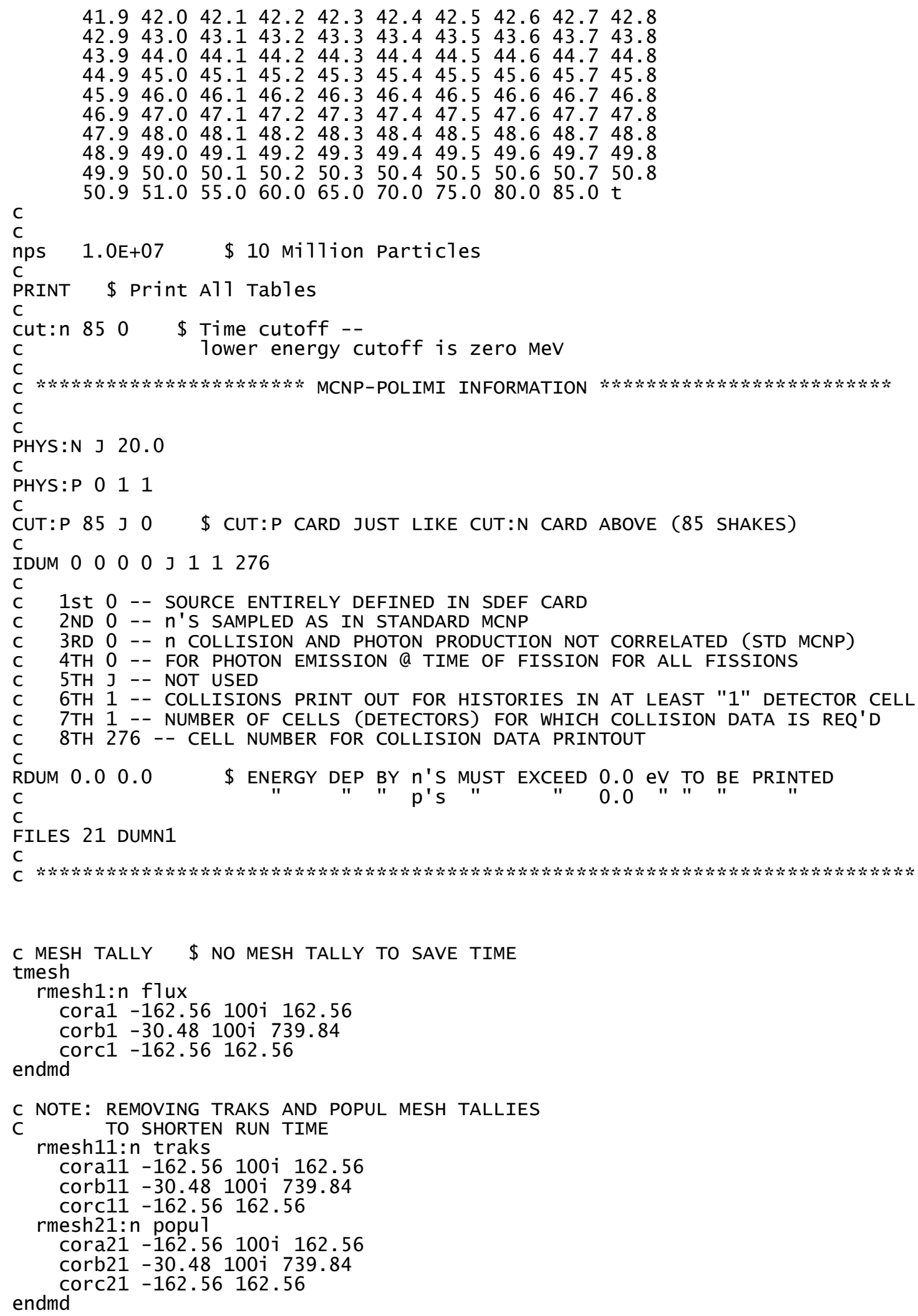




\section{APPENDIX B}

\section{THE nTOF POST-PROCESSING CODE}

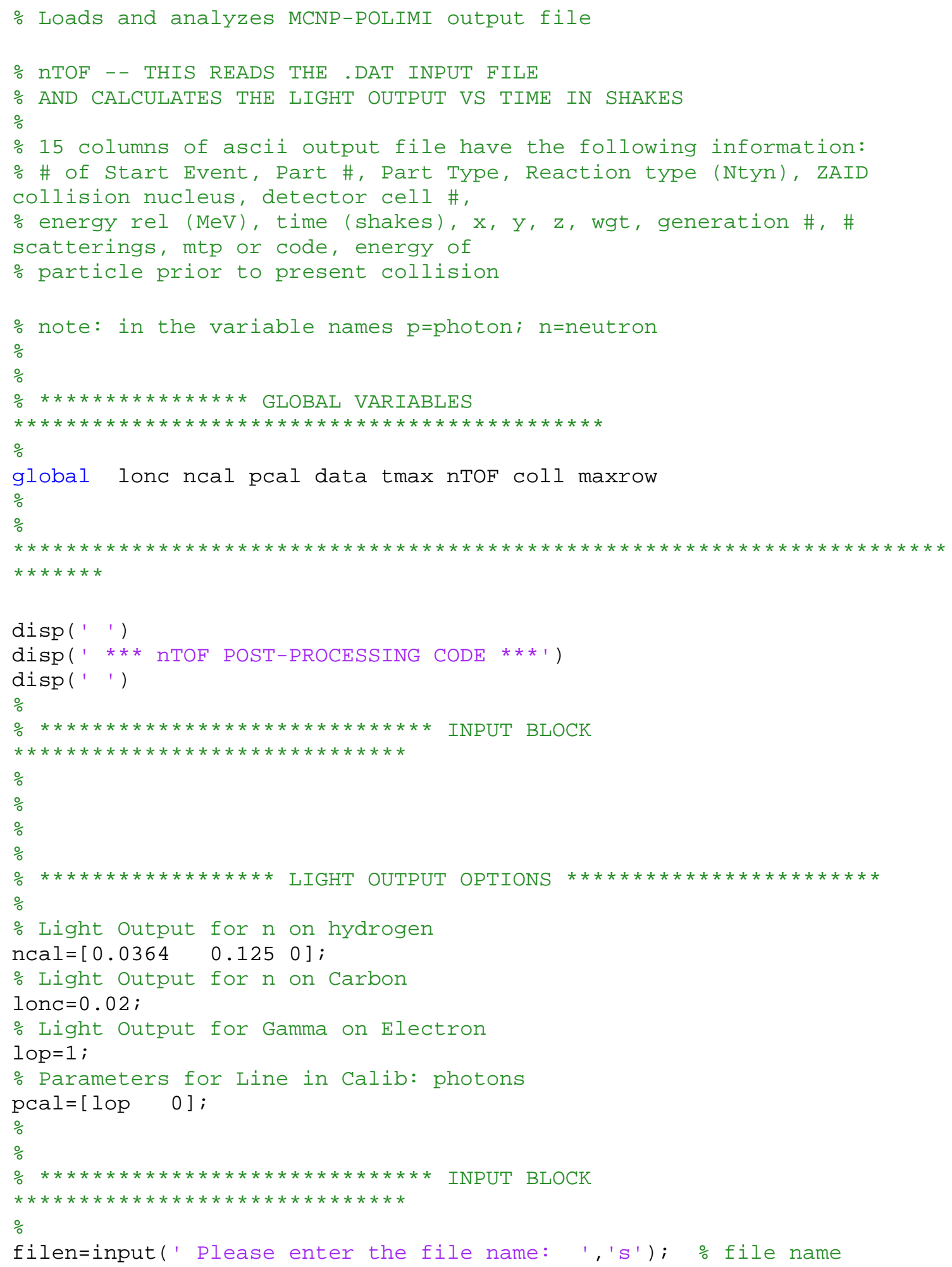




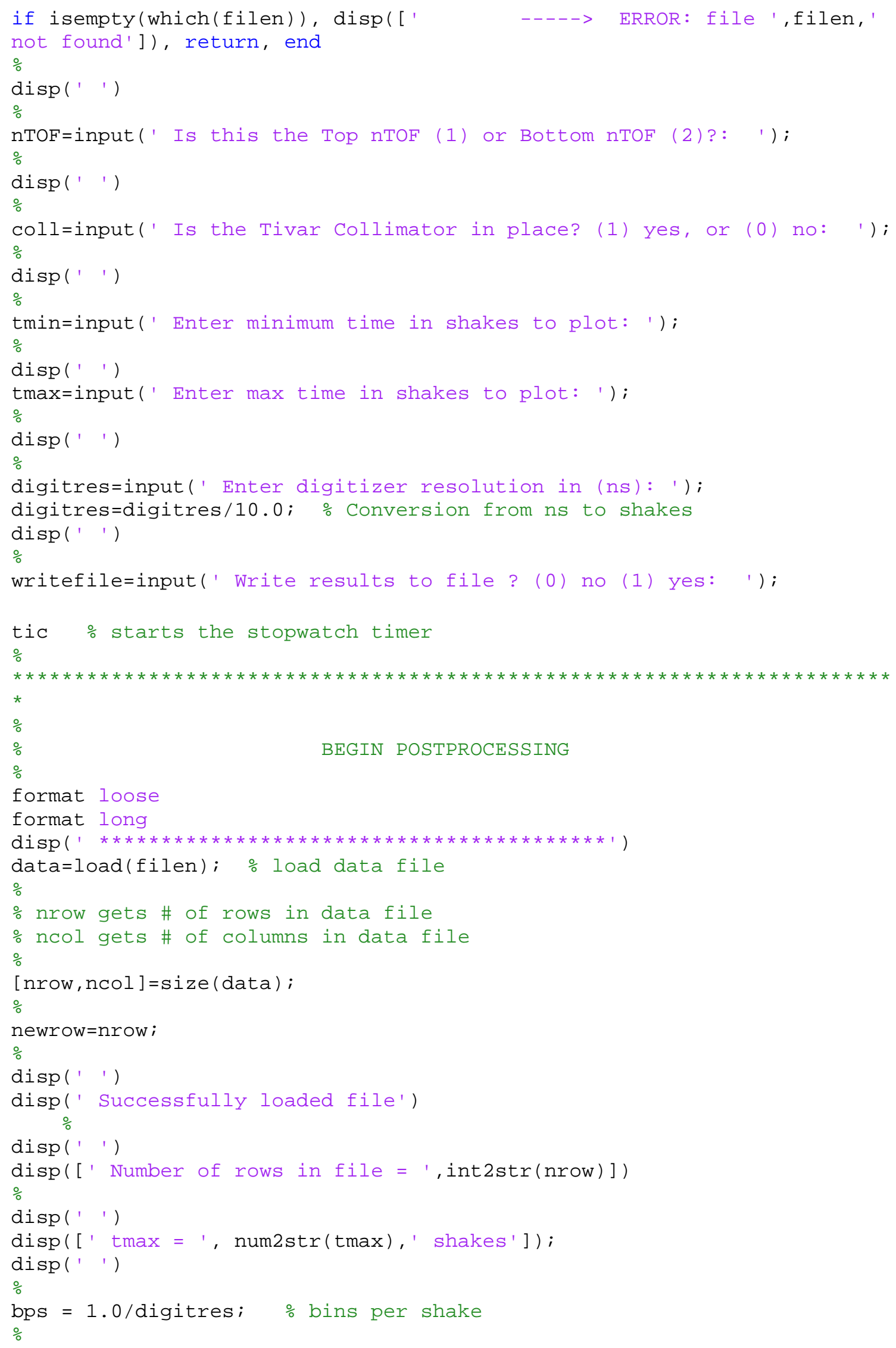




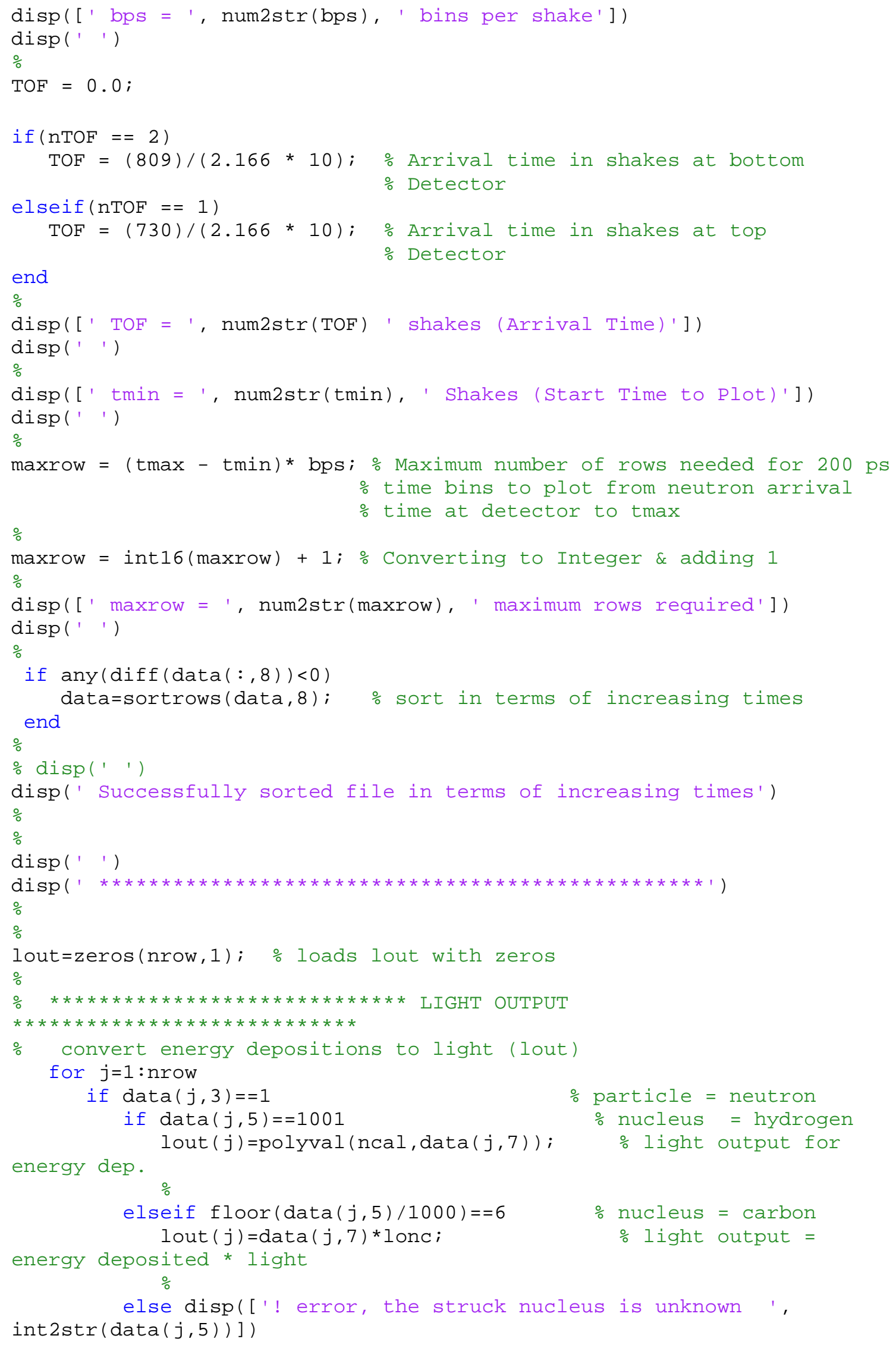




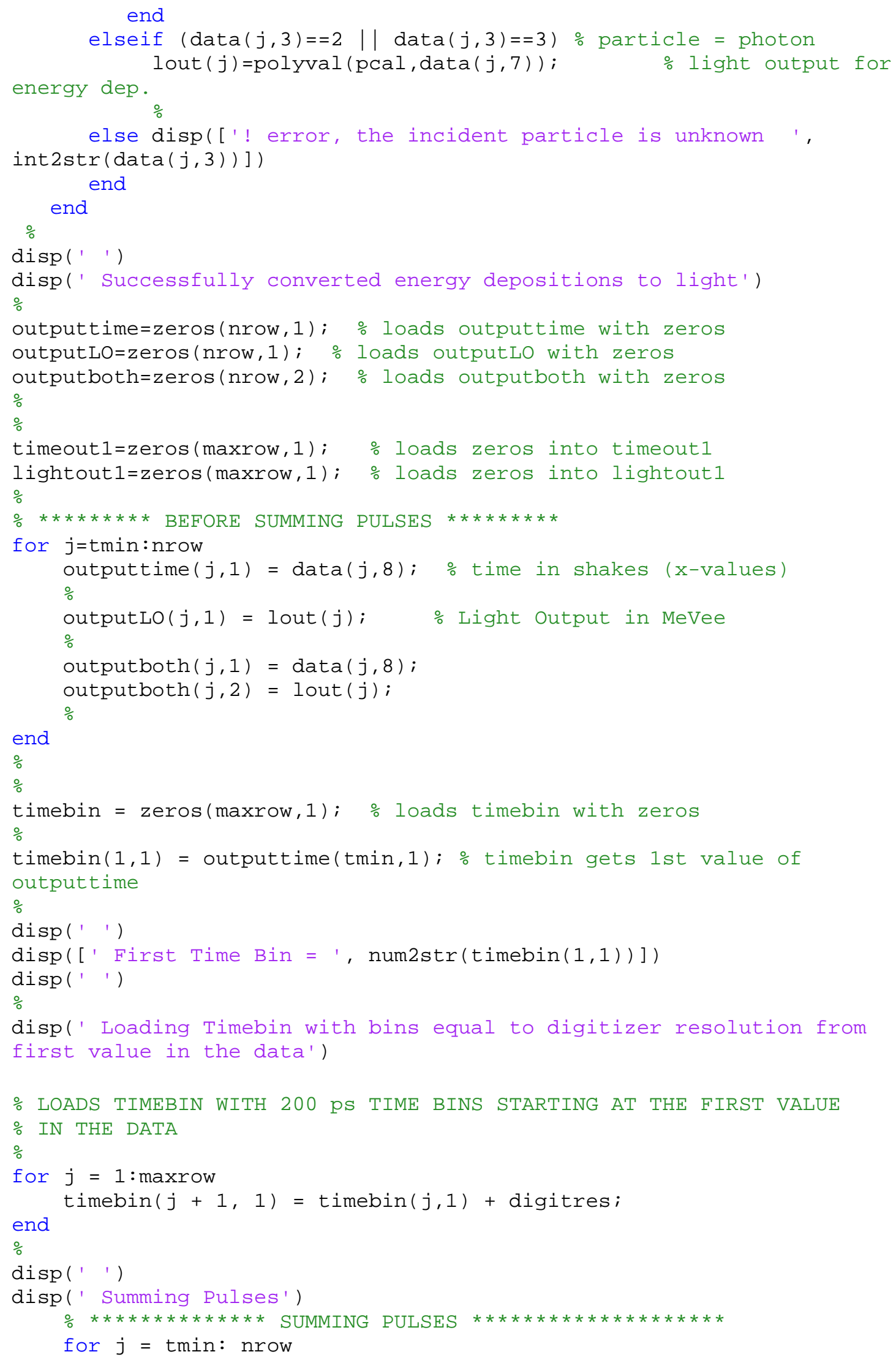




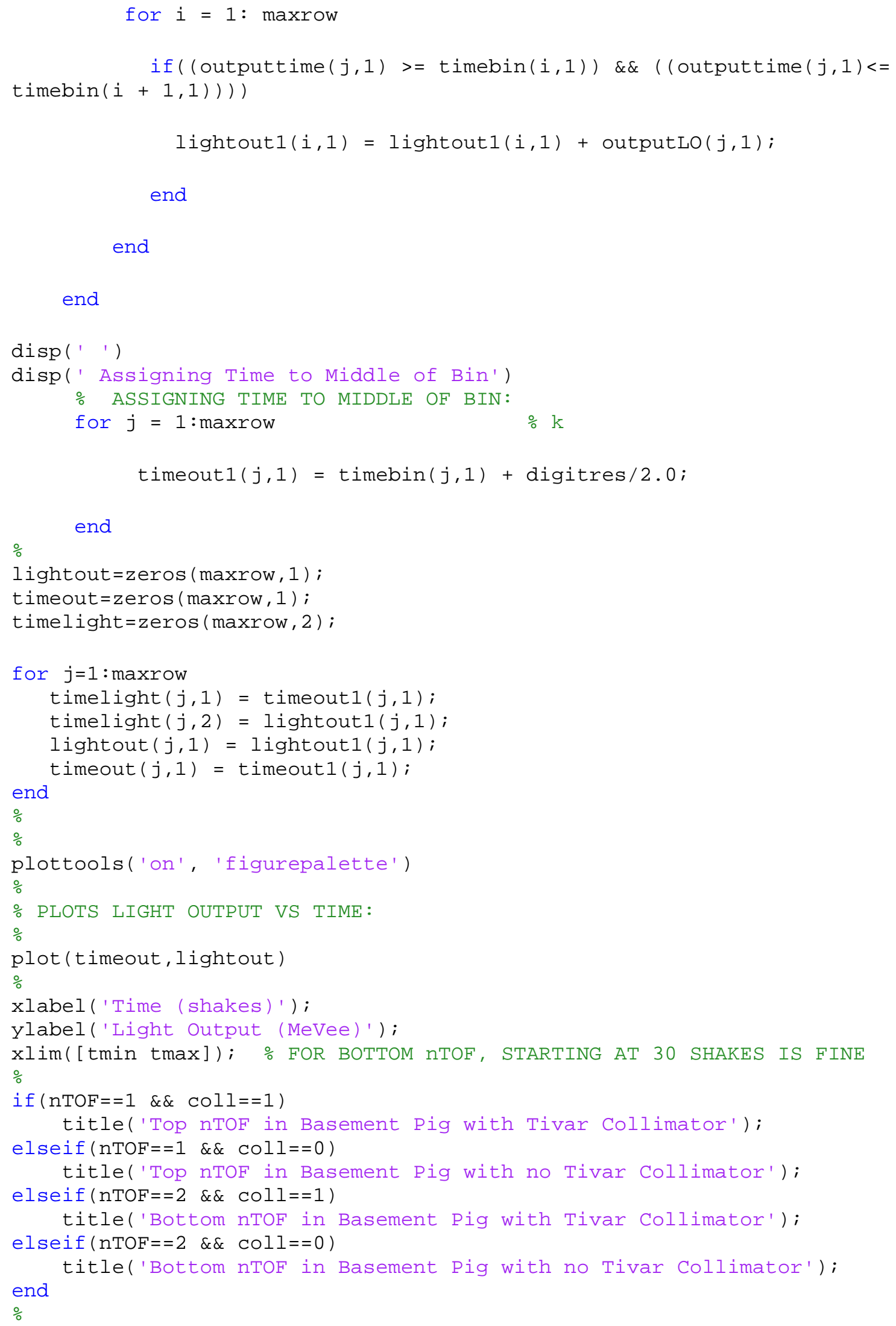




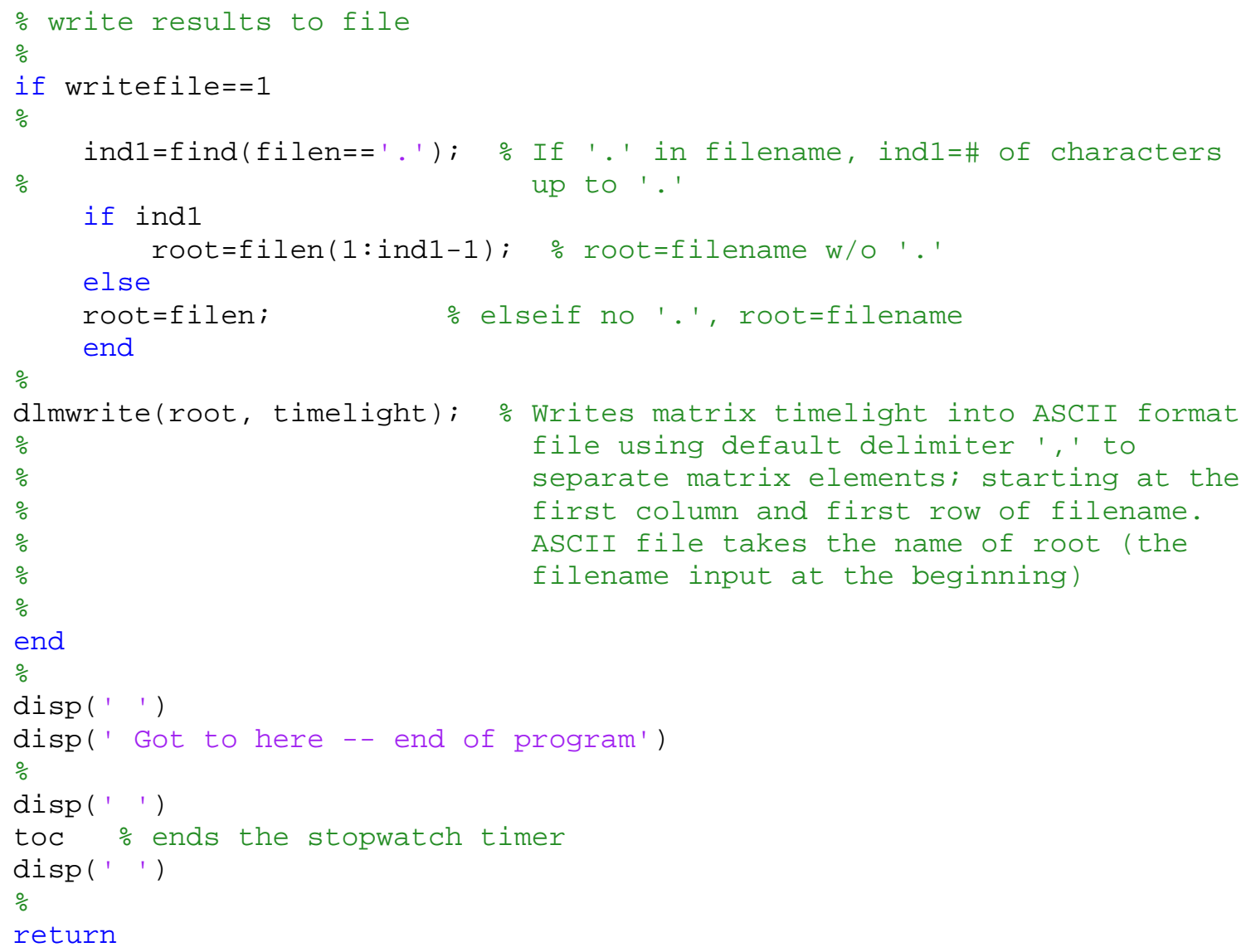




\section{APPENDIX C}

\section{THE CONVOLUTION (“FOLDING IN”) CODE}

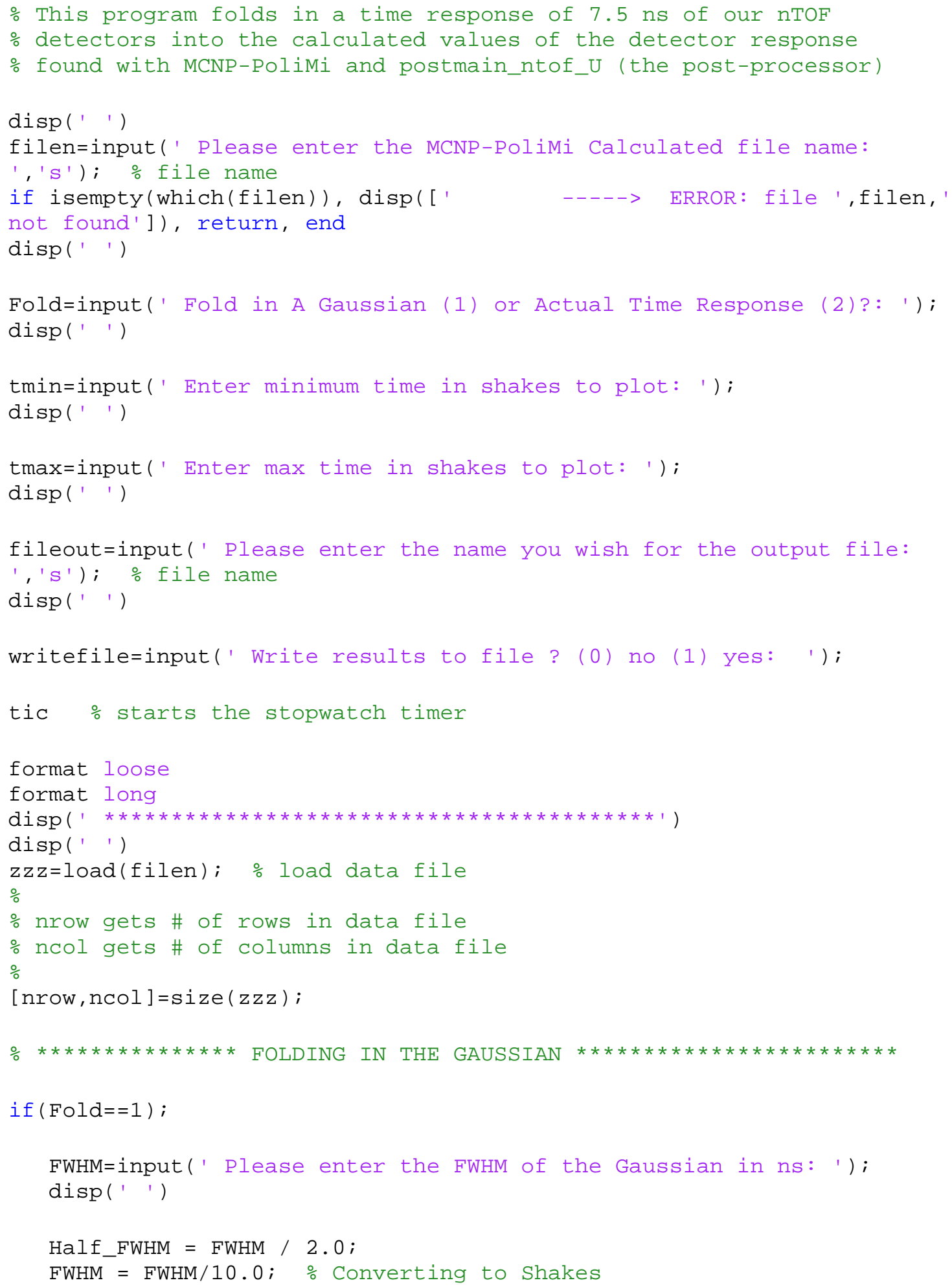




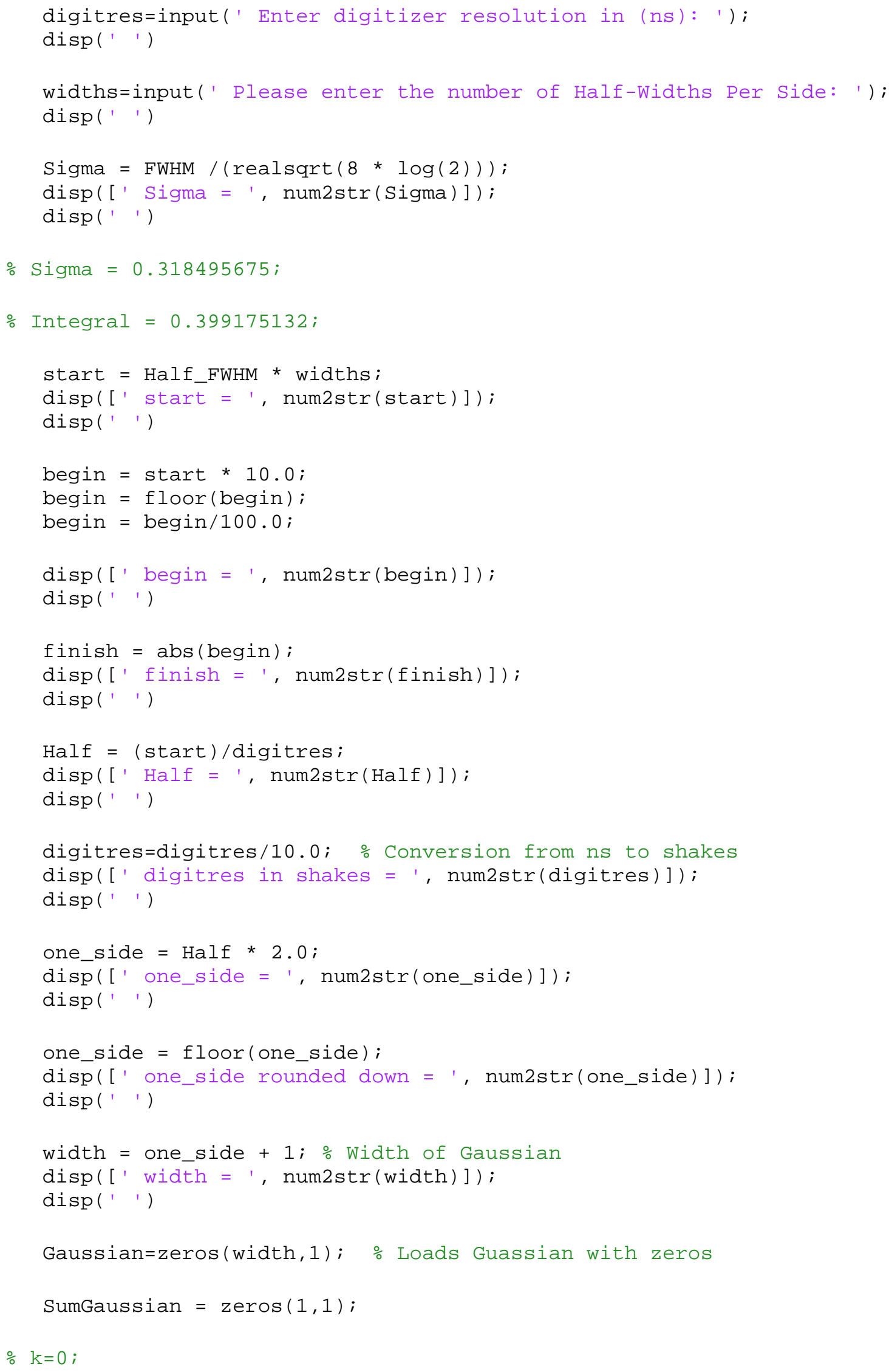




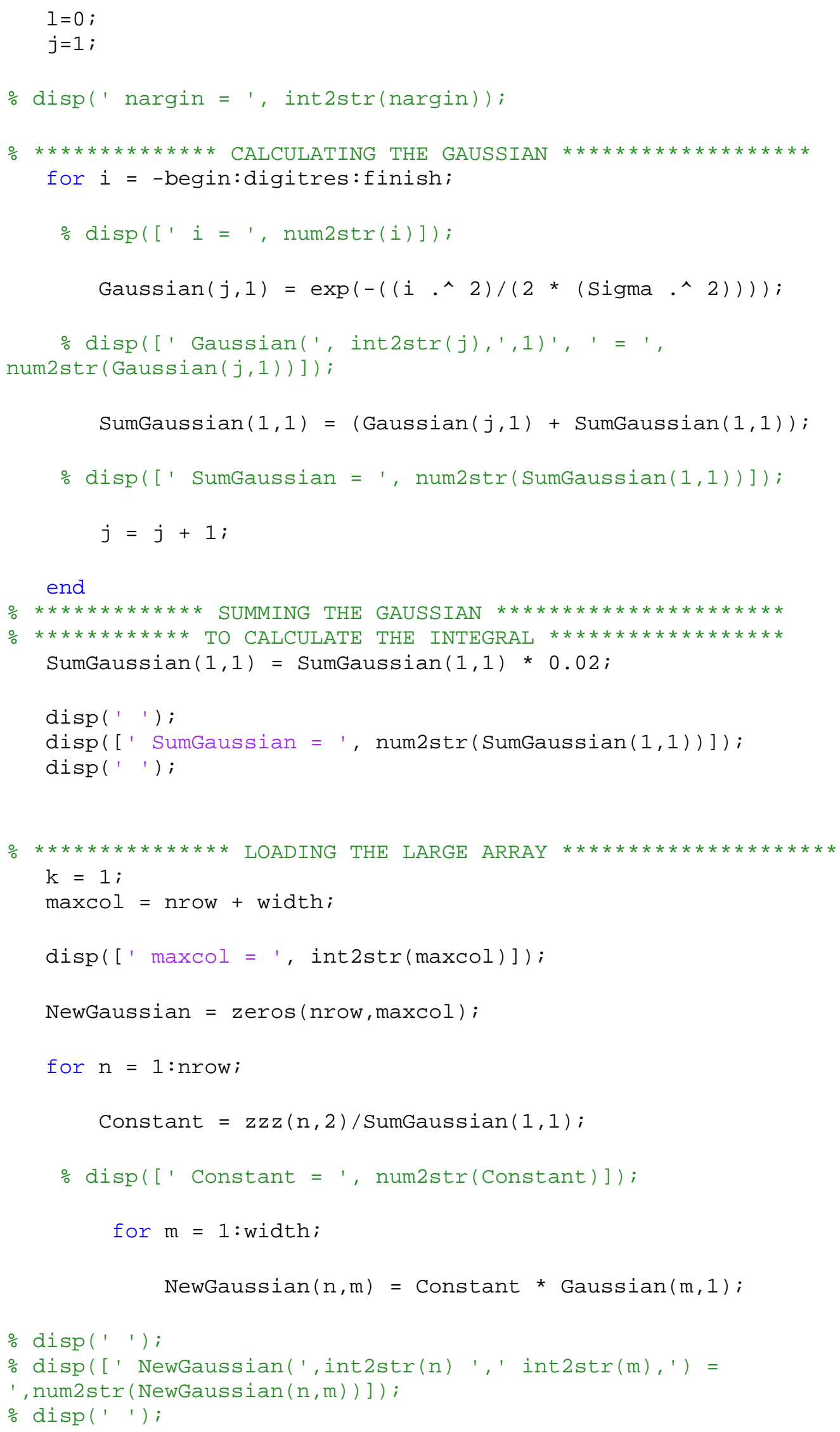




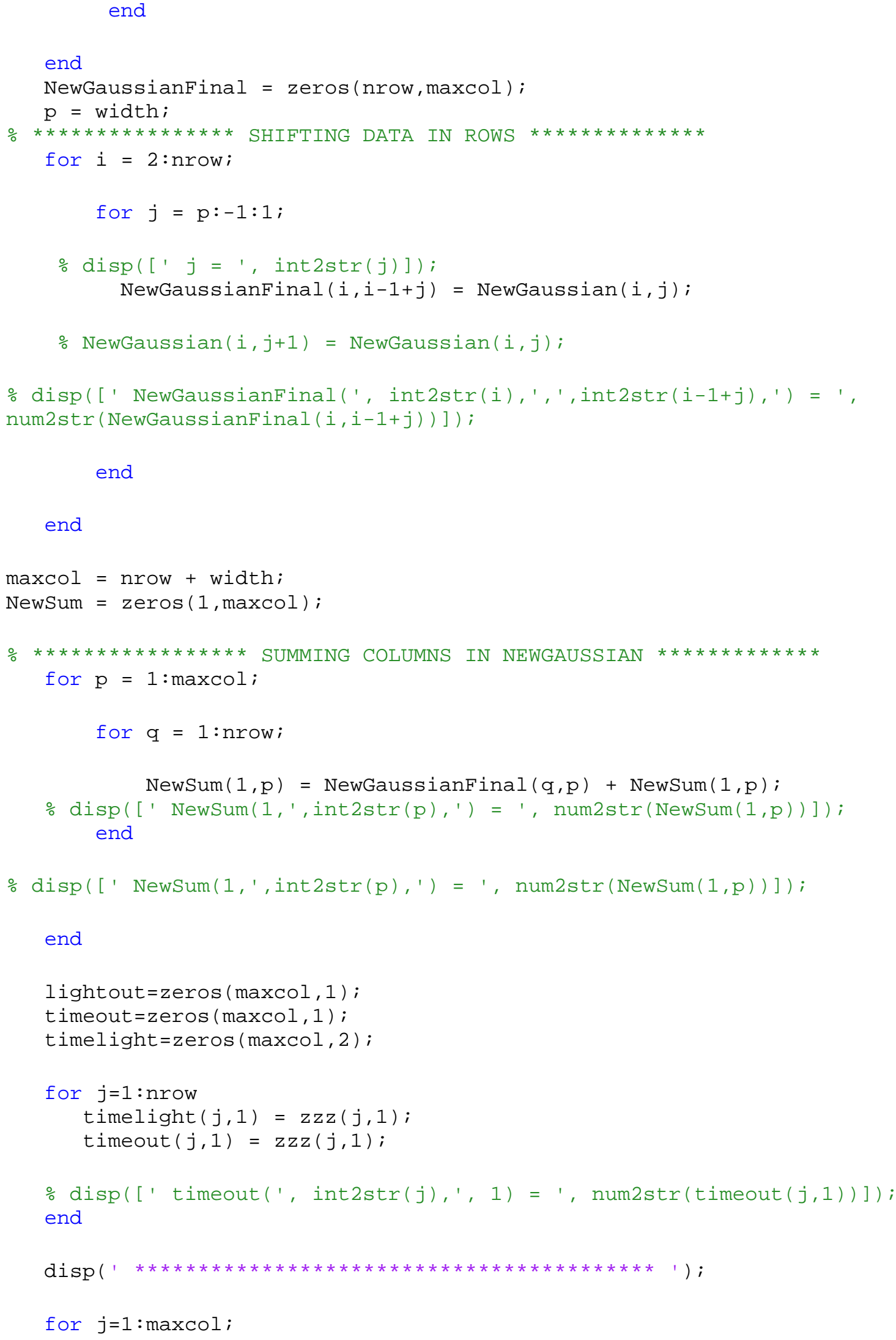




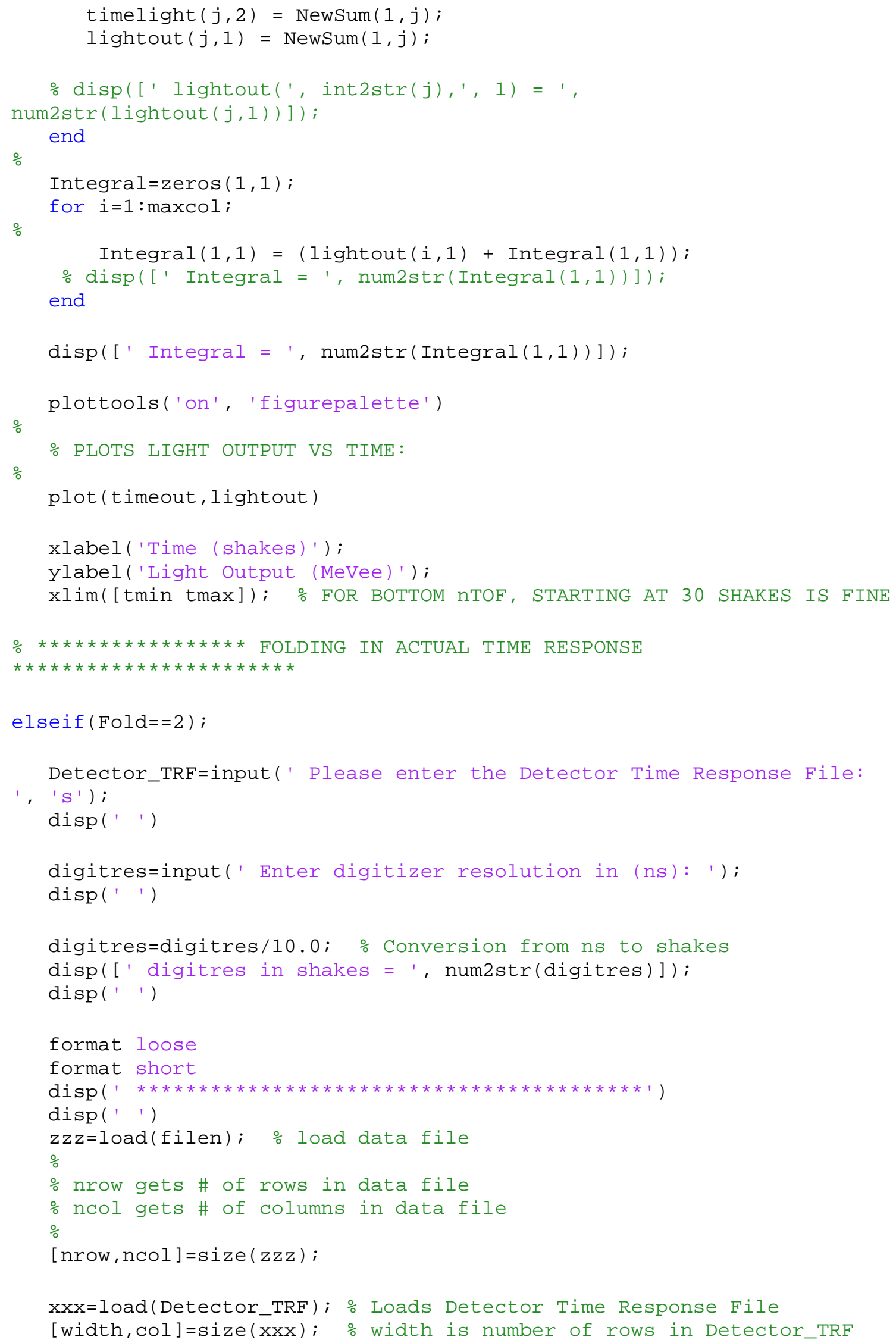




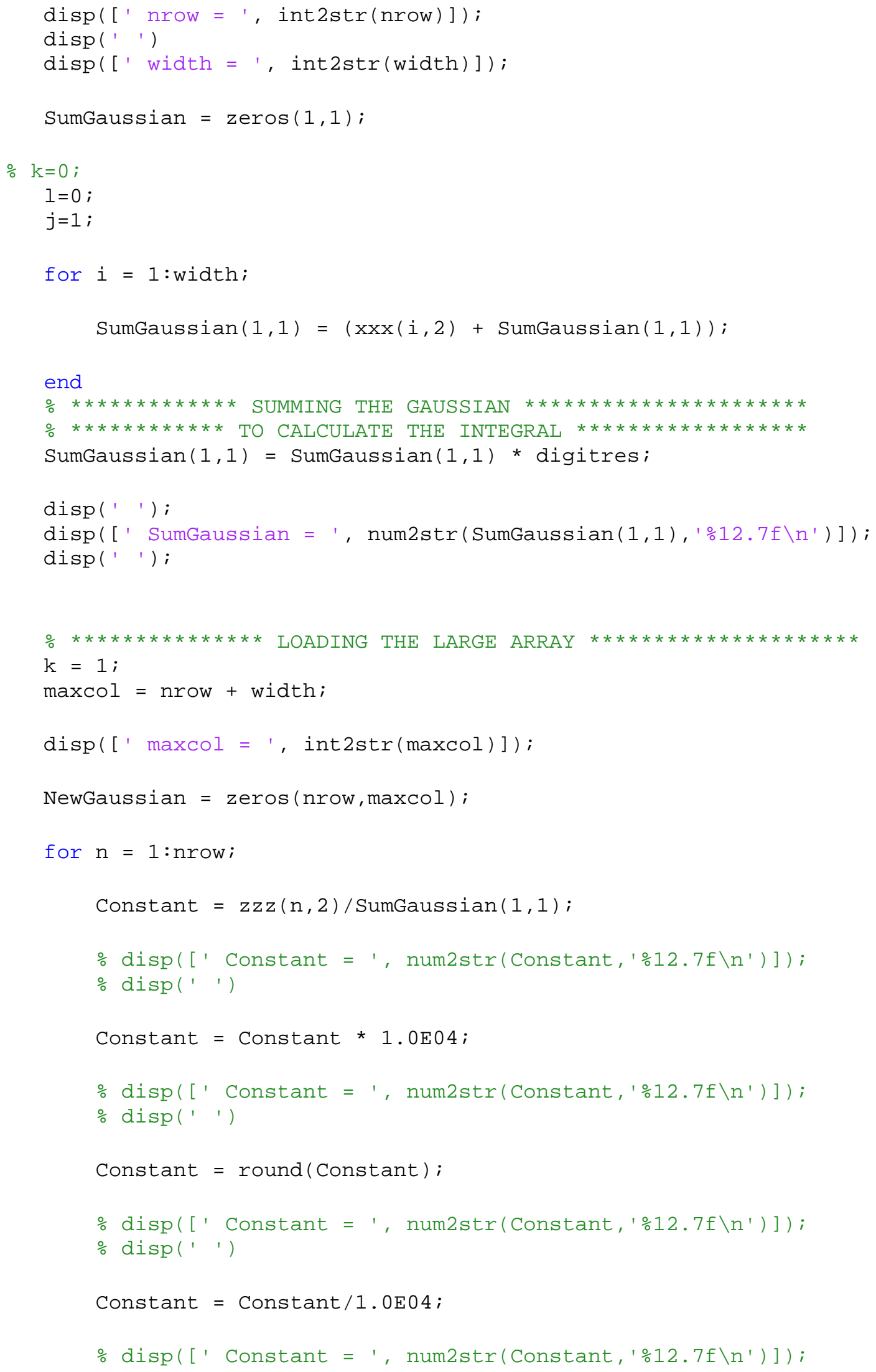




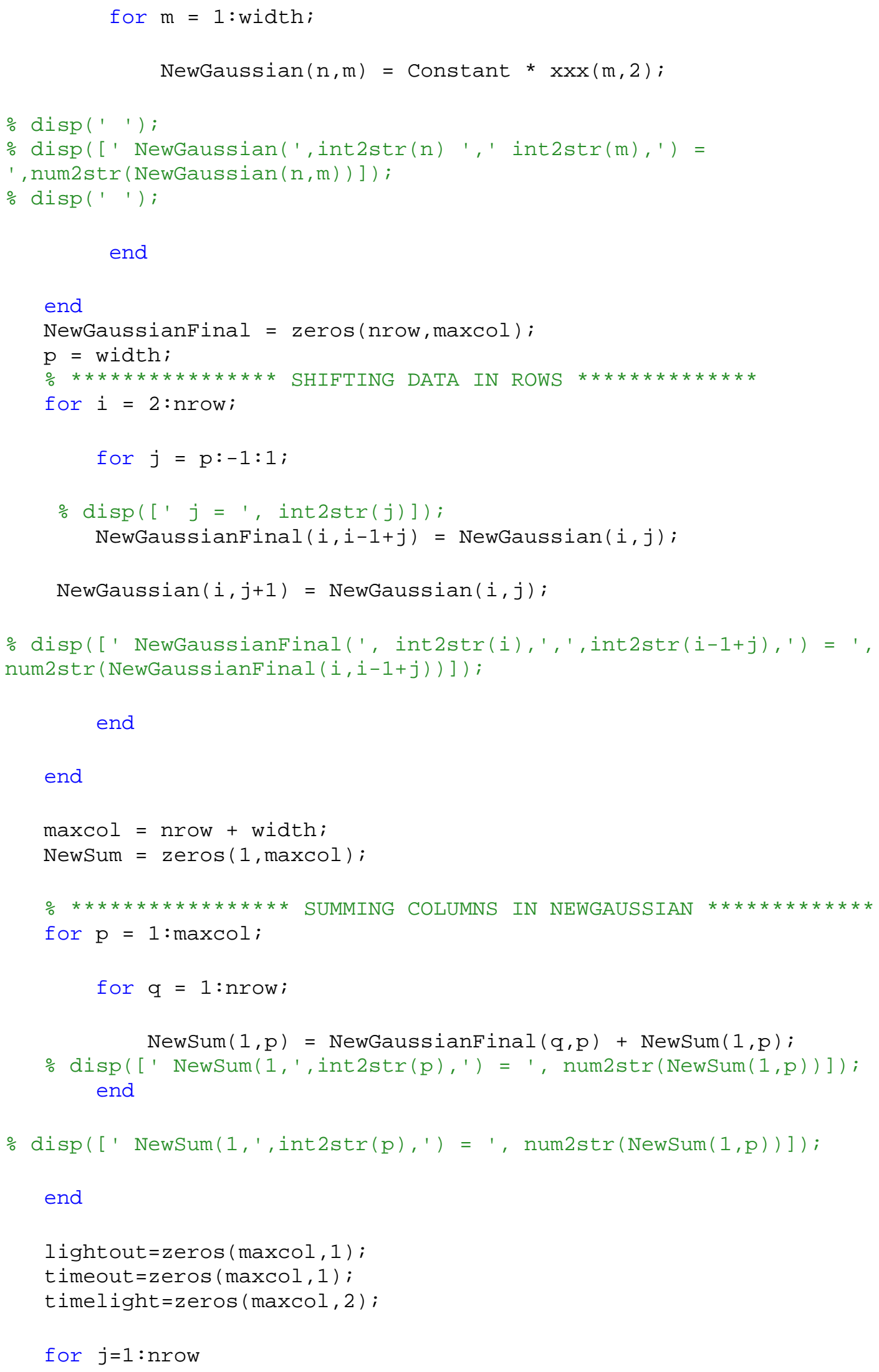




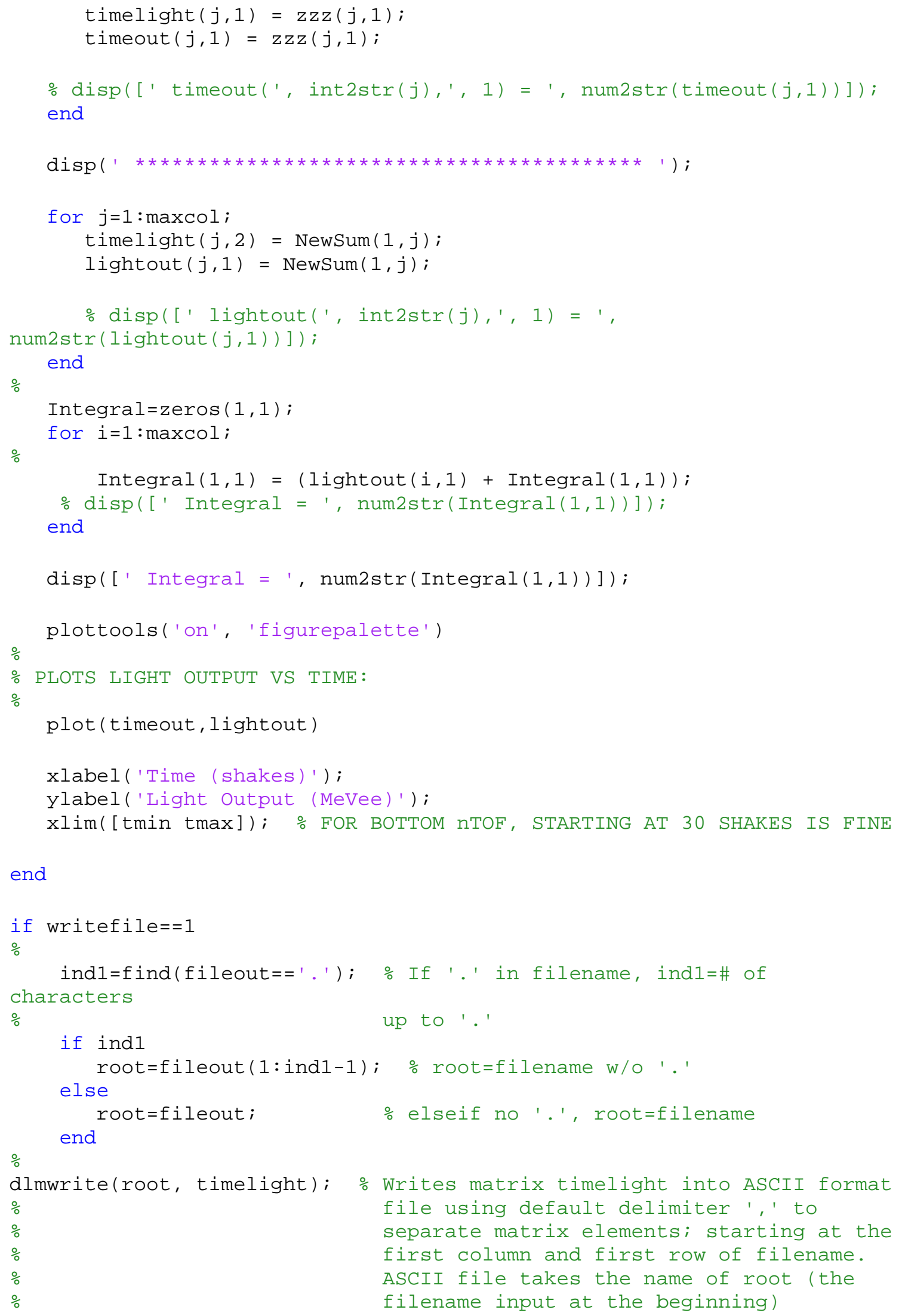




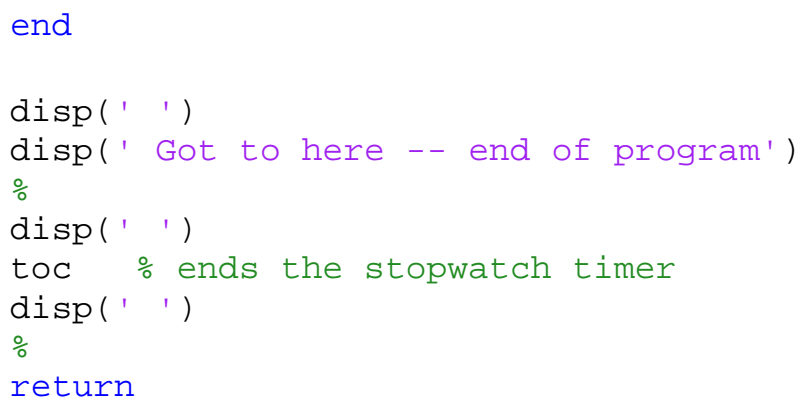


APPENDIX D

\section{THE DECONVOLUTION (“UNFOLDING”) CODE}

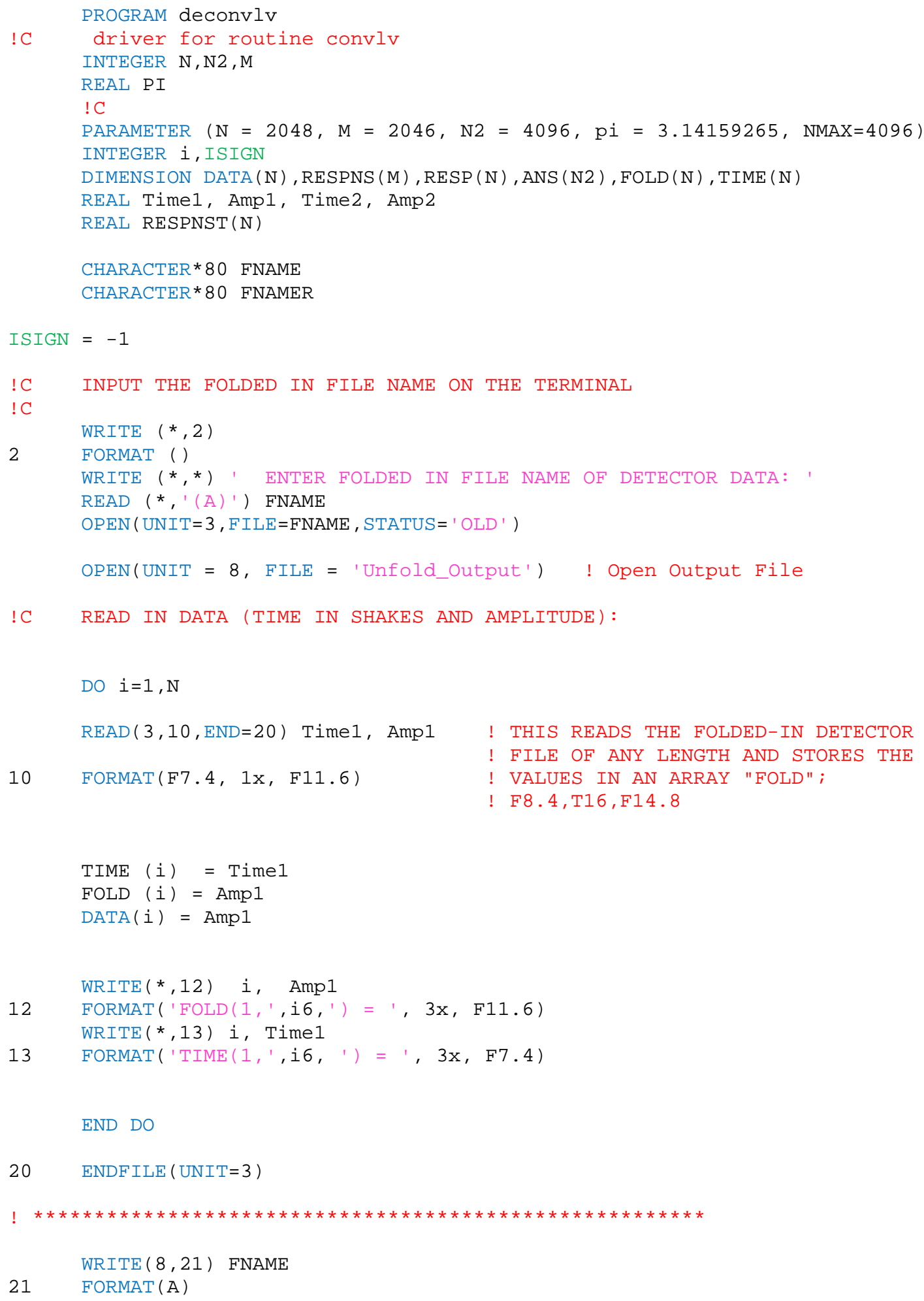




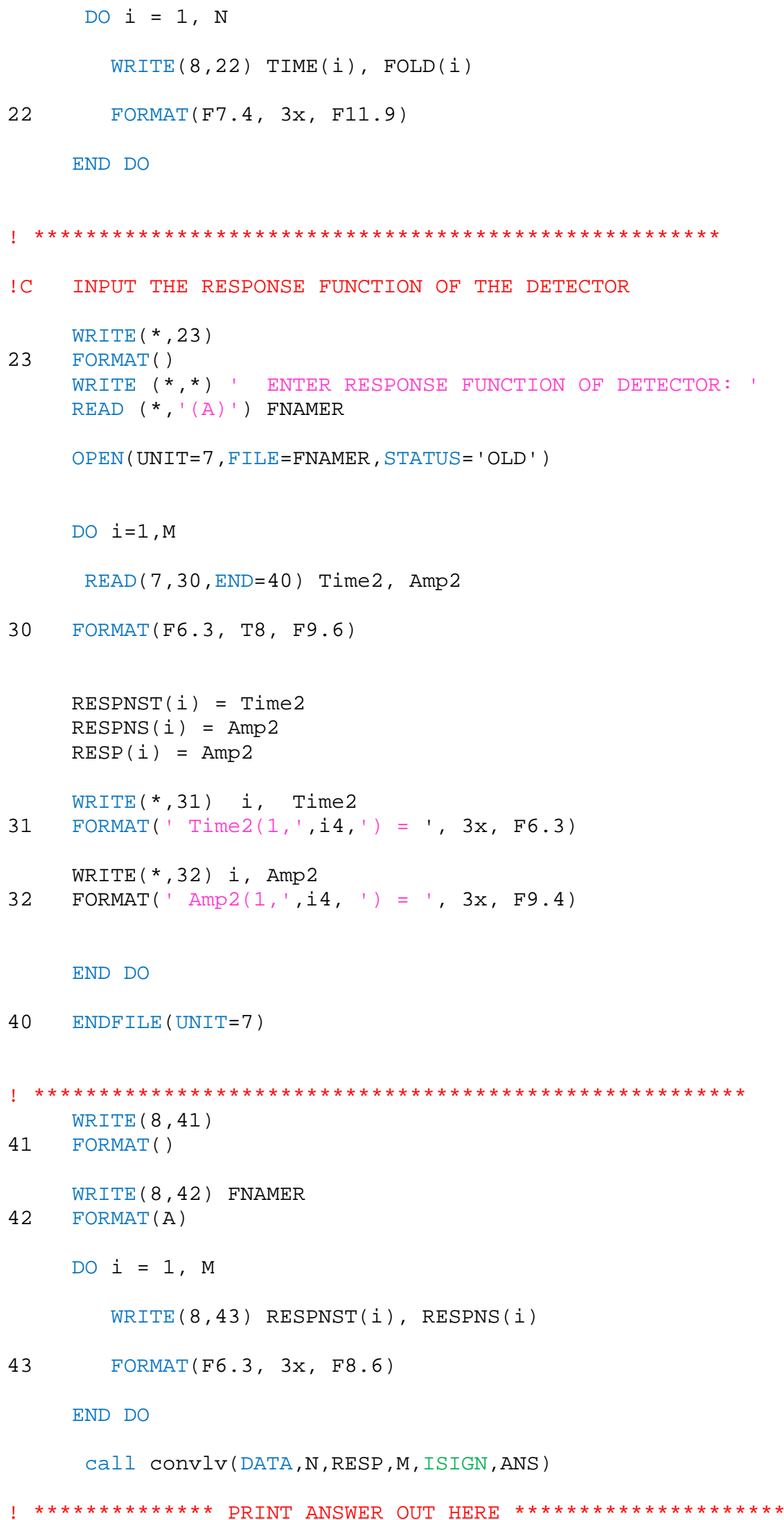




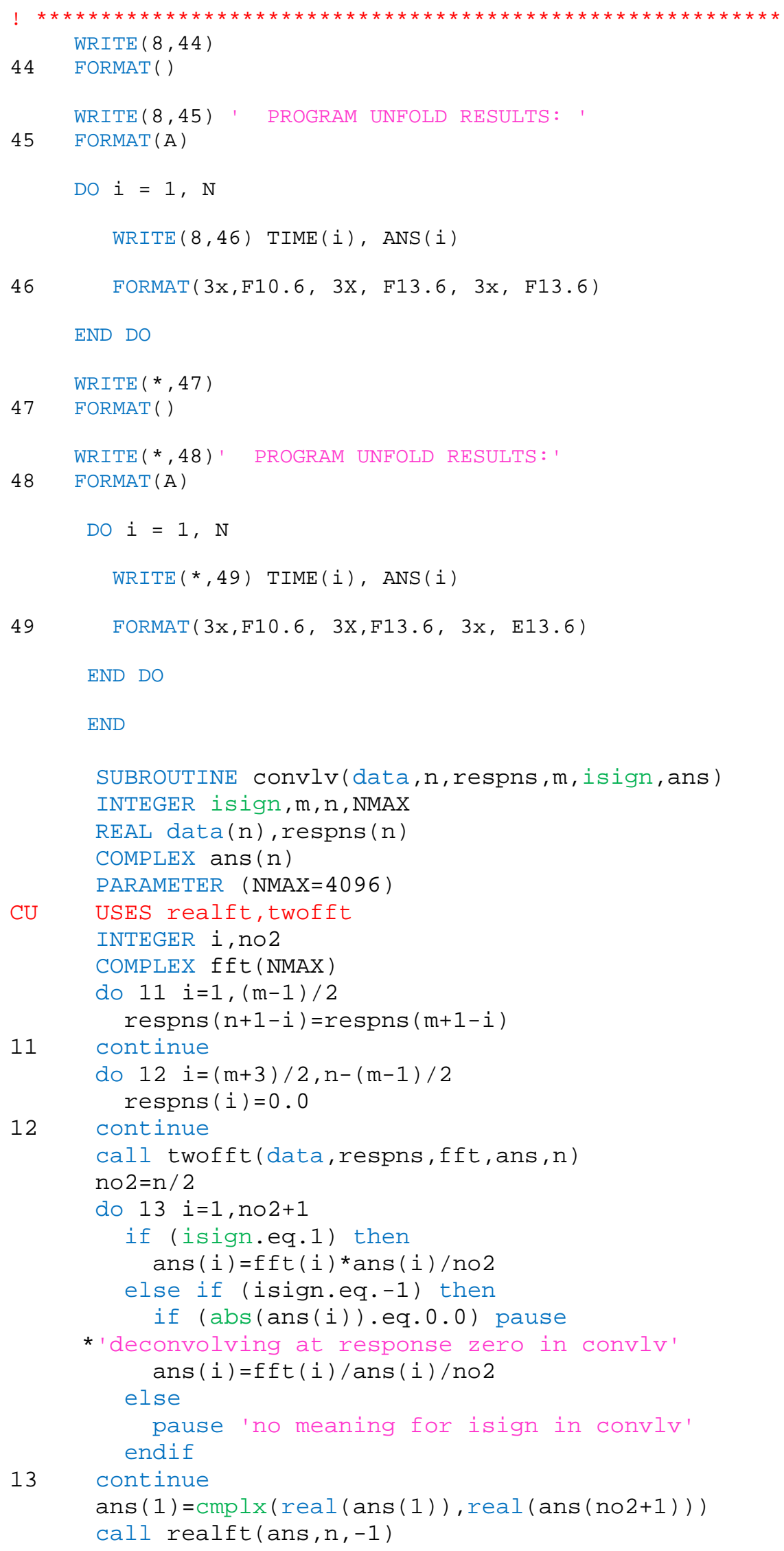




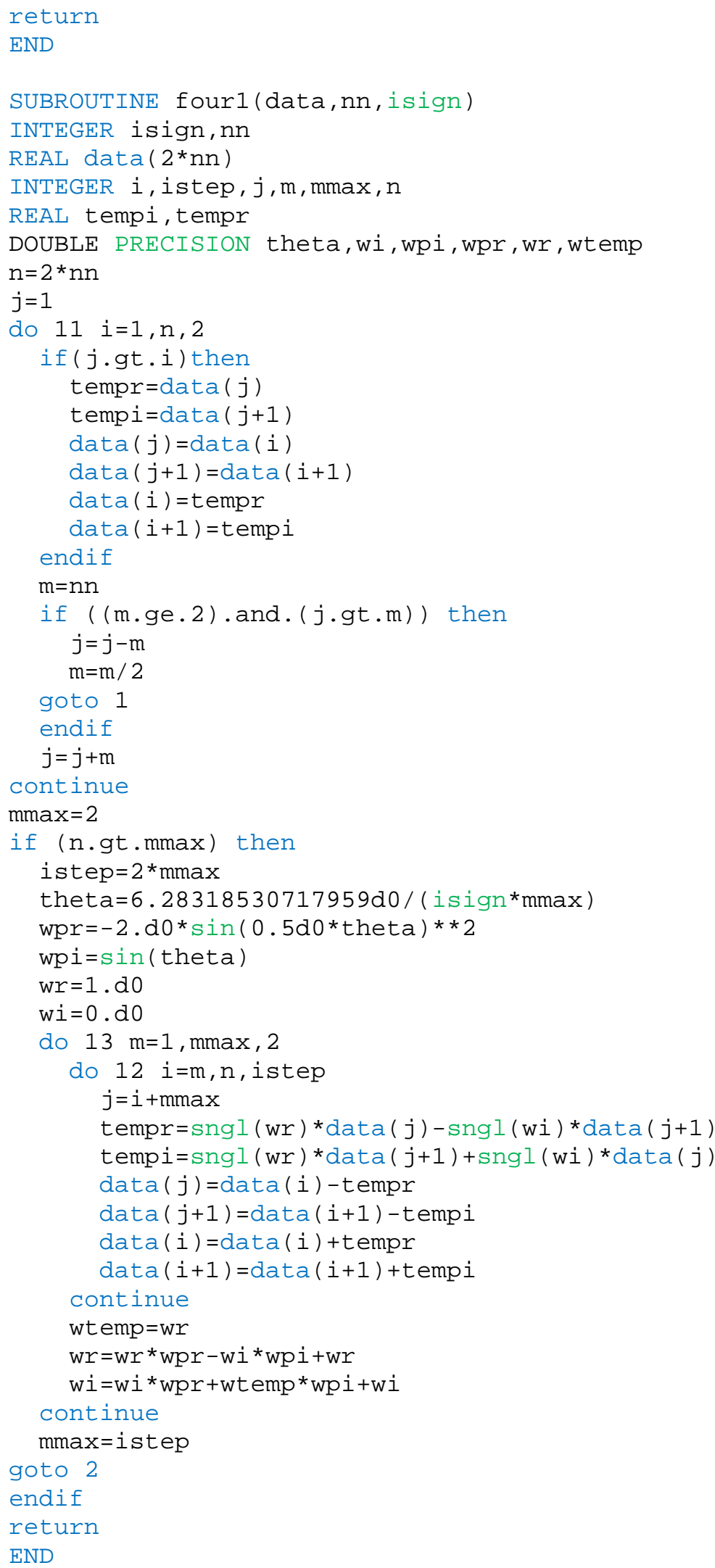




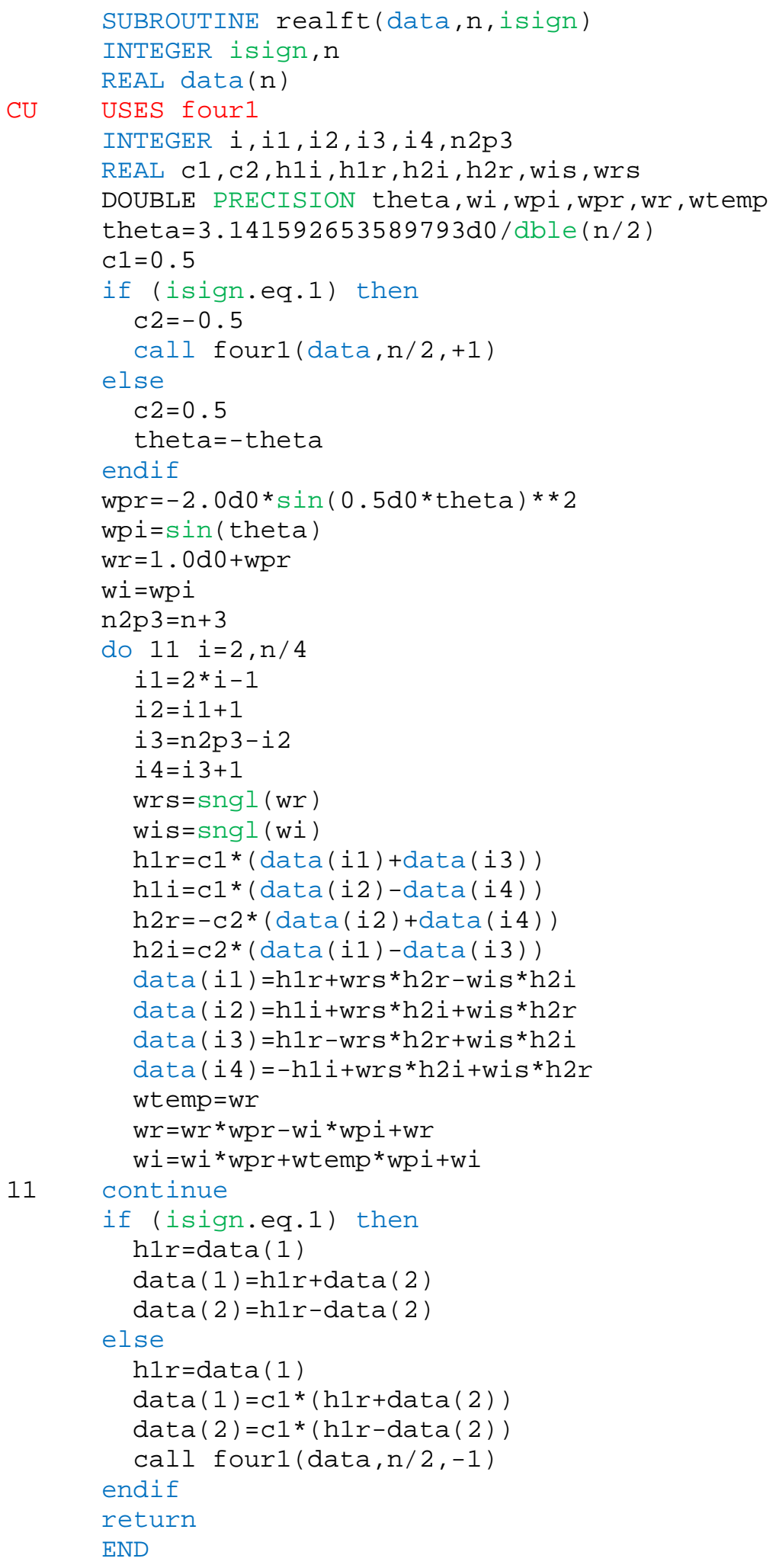




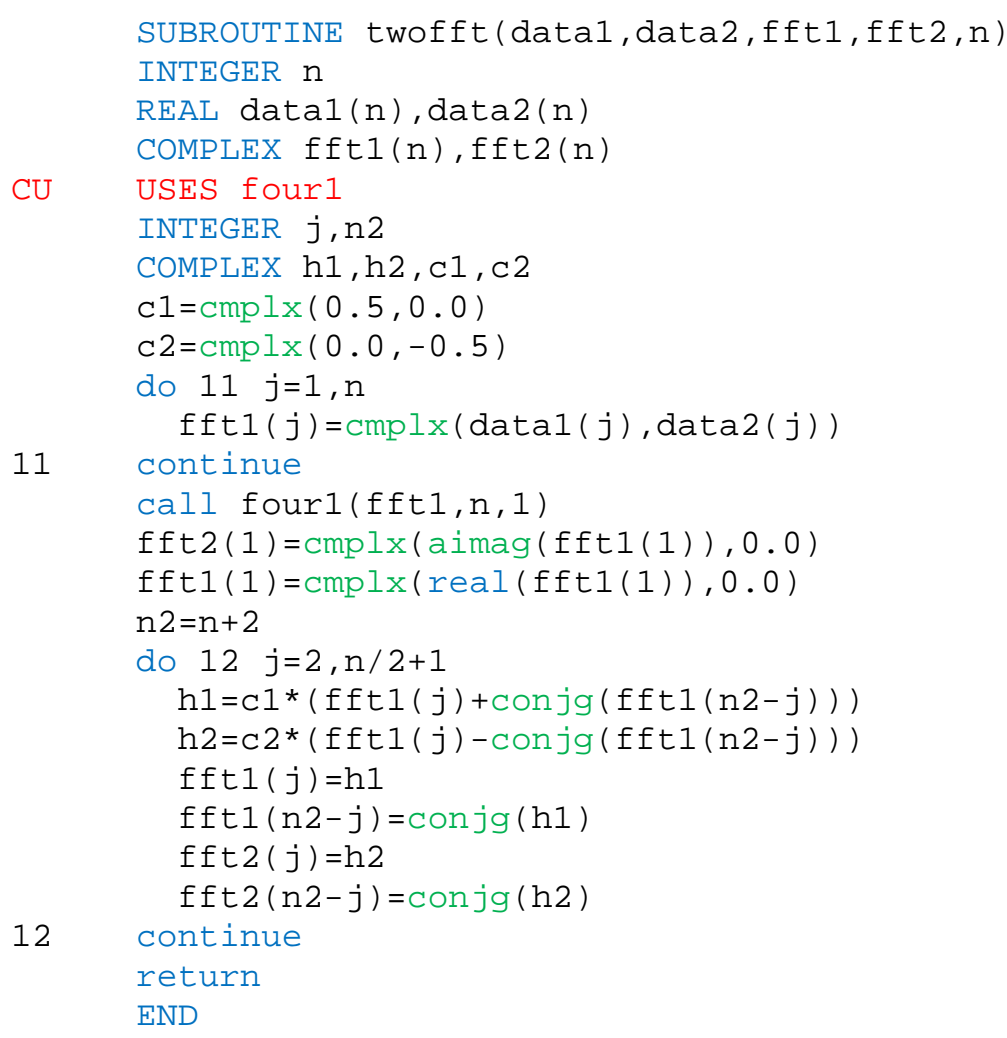




\section{APPENDIX E \\ IDAHO ACCELERATOR CENTER LAYOUT}

Shown in Figure 47 is the layout of the experiments that were performed at the Idaho Accelerator Center located at Idaho State University in Pocatello, Idaho [24]. The goal was to measure the time response of nTOF detectors using 50 ps pulses of $x$-rays. Initially the nTOFs were placed in the "Great Hall" which housed the $15 \mathrm{MeV}$ linac itself but the data had a high degree of background due to scattering, therefore the nTOFs were moved into the "Shielded Cell" - a room separated from the Great Hall by two $30.48 \mathrm{~cm}(1 \mathrm{ft})$ thick concrete walls separated by $1.2192 \mathrm{~m}(4 \mathrm{ft})$ of earth, through which

\section{Idaho Accelerator Center (IAC) Layout}

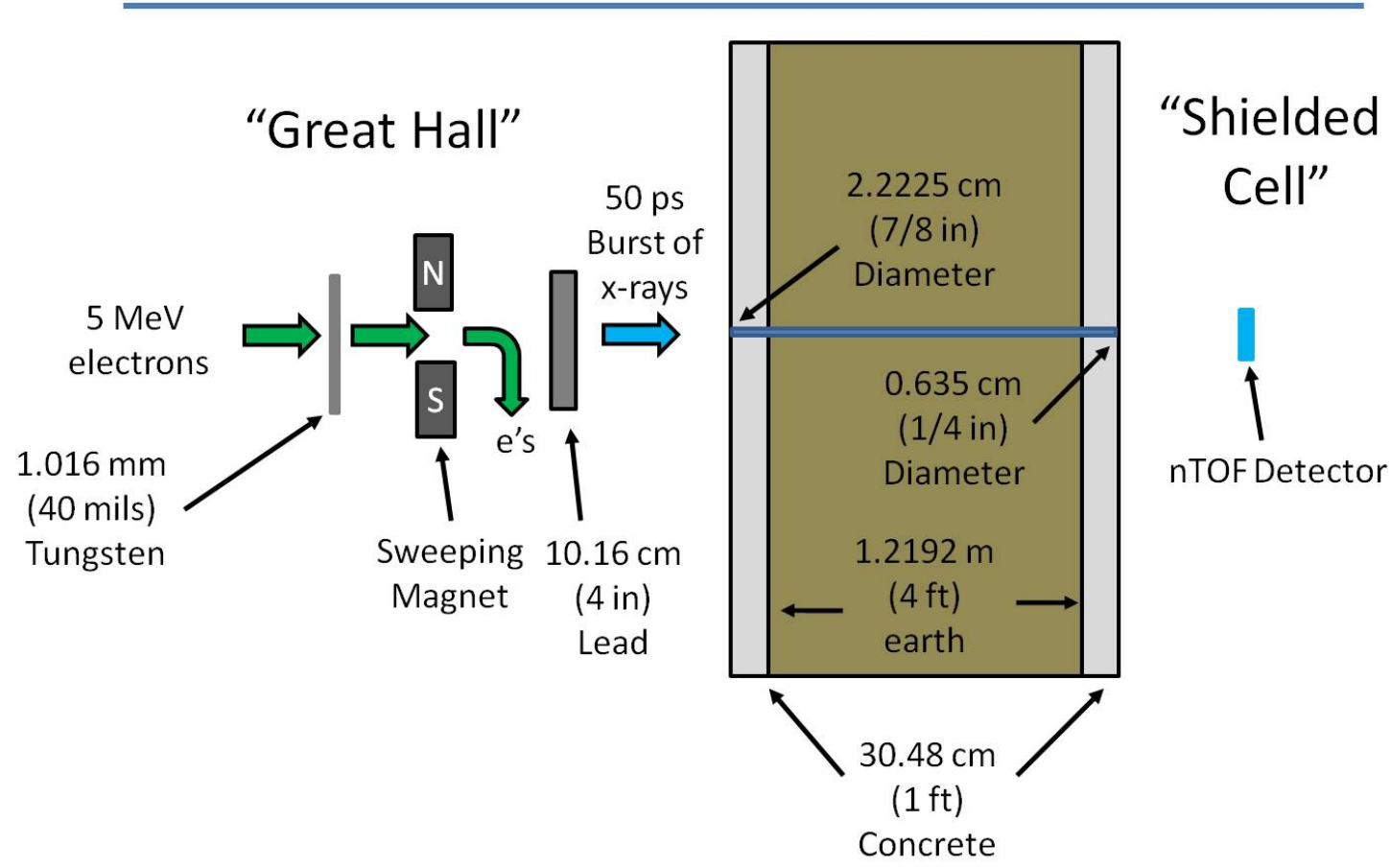

Figure 47. Idaho Accelerator Center (IAC) Layout. The "Great Hall" housed the $15 \mathrm{MeV}$ Linac. The nTOF detectors were placed in the "Shielded Cell" behind two $30.48 \mathrm{~cm}(1 \mathrm{ft})$ thick walls

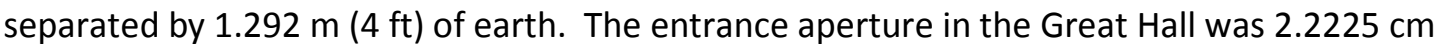
( $7 / 8$ in) diameter and the exit aperture in the Shielded Cell was $0.635 \mathrm{~cm}(1 / 4 \mathrm{in})$. This geometry provided good data with very little background. The goal was to measure the time response of the detectors using 50 ps bursts of $x$-rays. 
a narrow collimator ran from the Great Hall into the Shielded Cell. The entrance aperture of the collimator was $2.2225 \mathrm{~cm}(7 / 8 \mathrm{in})$ and the exit aperture was $0.635 \mathrm{~cm}$ (1/4 in). The data obtained from the nTOFs placed in the Shielded Cell was quite good, and shown in Figure 48.

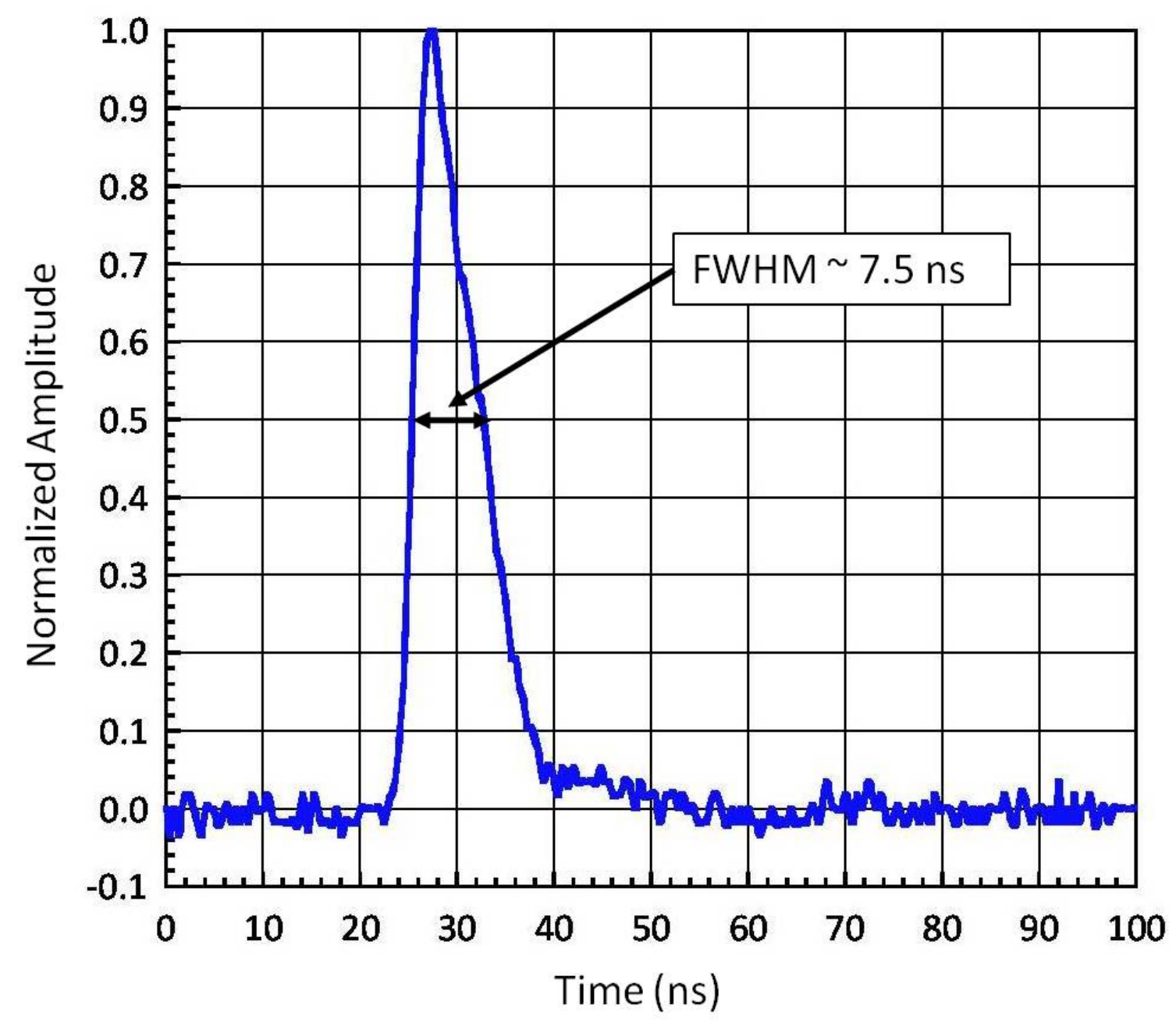

Figure 48. Experimental Time response found at the Idaho Accelerator Center (IAC) using 50 ps bursts of x-rays. The full width at half maxima is approximately $7.5 \mathrm{~ns}$. This data was obtained in the "Shielded Cell" (see Figure 47) where the background was quite low due to the extensive amount of shielding and narrow collimation.

It was found that the best results were obtained when the linac operated at 400 $\mathrm{mA}$, and pulsed $5 \mathrm{MeV}$ electrons into $1.016 \mathrm{~mm}$ (40 mils) of tungsten. A sweeping 
magnet removed the electrons out of the beam, leaving only the 50 ps bursts of $x$-rays which were attenuated by $10.16 \mathrm{~cm}$ (4 in) of lead. The x-rays were collimated through

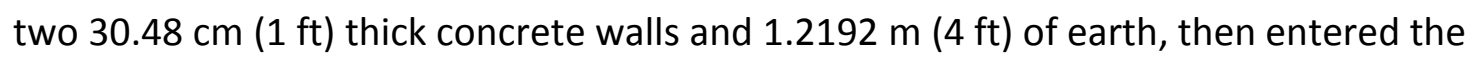
shielded cell. The data was recorded on a Tektronix TDS7254B $2.5 \mathrm{GHz}, 4$ channel digital phosphor digitizer [19] in an average over several pulses (typically 32). In this way, the time response of four nTOF detectors was found with a nominal value of a full width at half maxima to be 7.5 ns. 


\section{APPENDIX $F$ \\ NEW COLLIMATOR DESIGN}

In the 2006 - 2007 timeframe, the Z-Machine was refurbished, and attention was paid to upgrading all diagnostics. It was known that the addition of the collimator under target chamber center greatly improved neutron signals as shown in this work; however, there was room for improvement. Therefore, a design of a new collimator was undertaken, to have a great deal more mass than the first one. Figure 49 shows the model of the first collimator that was shown to improve signals on shot z1549.

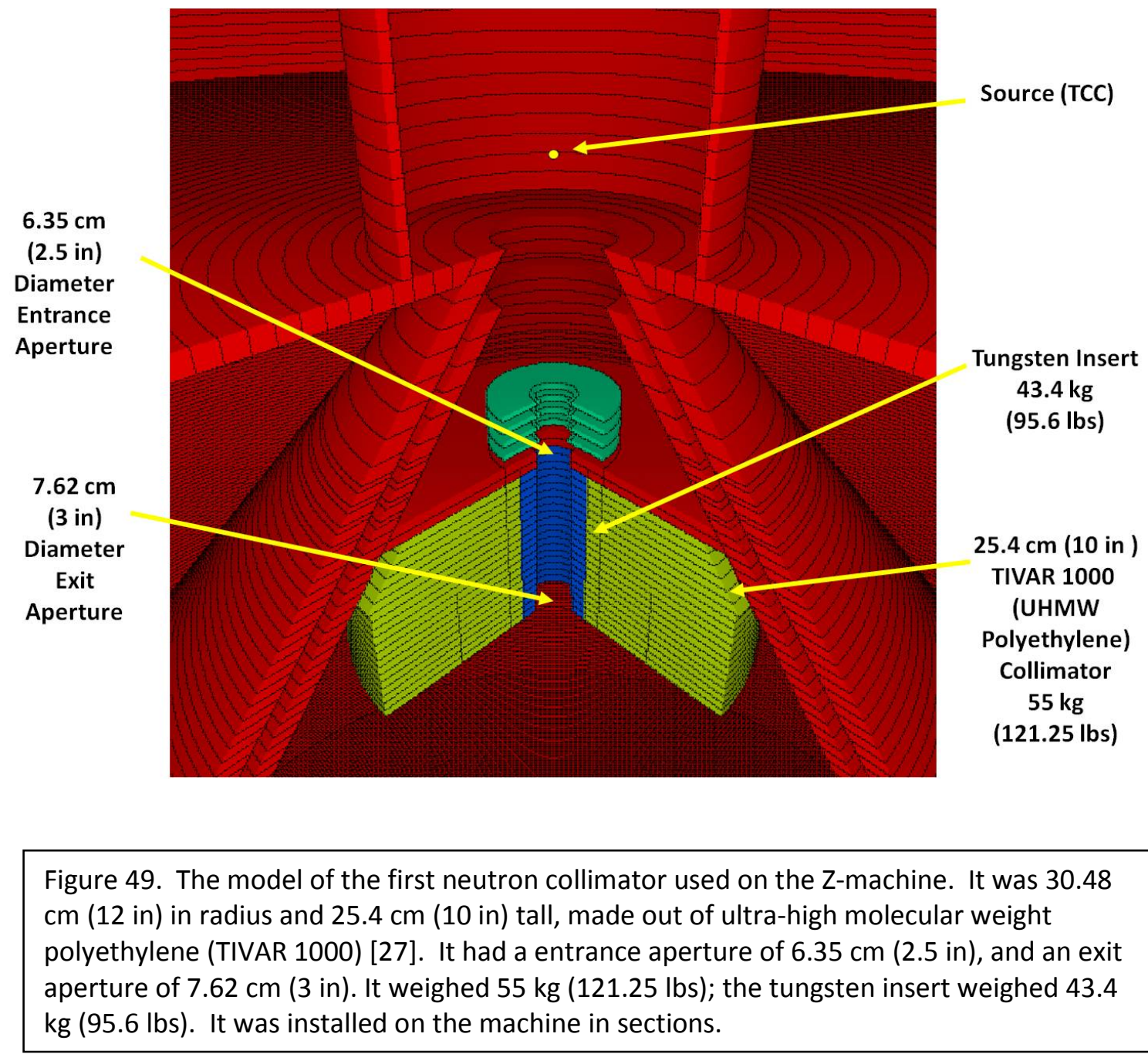


Figure 50 shows the design of the new collimator. The radius was increased the top radius is $24.75 \mathrm{~cm}$ (9.74 in) and the bottom radius is $45 \mathrm{~cm}$ (17.7 in). Also, a cylinder of ultra-high molecular weight polyethylene (TIVAR 1000) [27] $38.1 \mathrm{~cm}$ (15 in) was added along the axis, giving it a mushroom appearance. The aperture along the Zaxis was $3.81 \mathrm{~cm}(1.5 \mathrm{in}$ ) in diameter (note that this is half the diameter of the previous collimator's exit aperture, or $7.62 \mathrm{~cm}(3 \mathrm{in}))$. The mass of the new collimator was 109.5 $\mathrm{kg}(241.3 \mathrm{lbs})$, and the mass of the tungsten insert was $8.3 \mathrm{~kg}(18.4 \mathrm{lbs})$. Since the

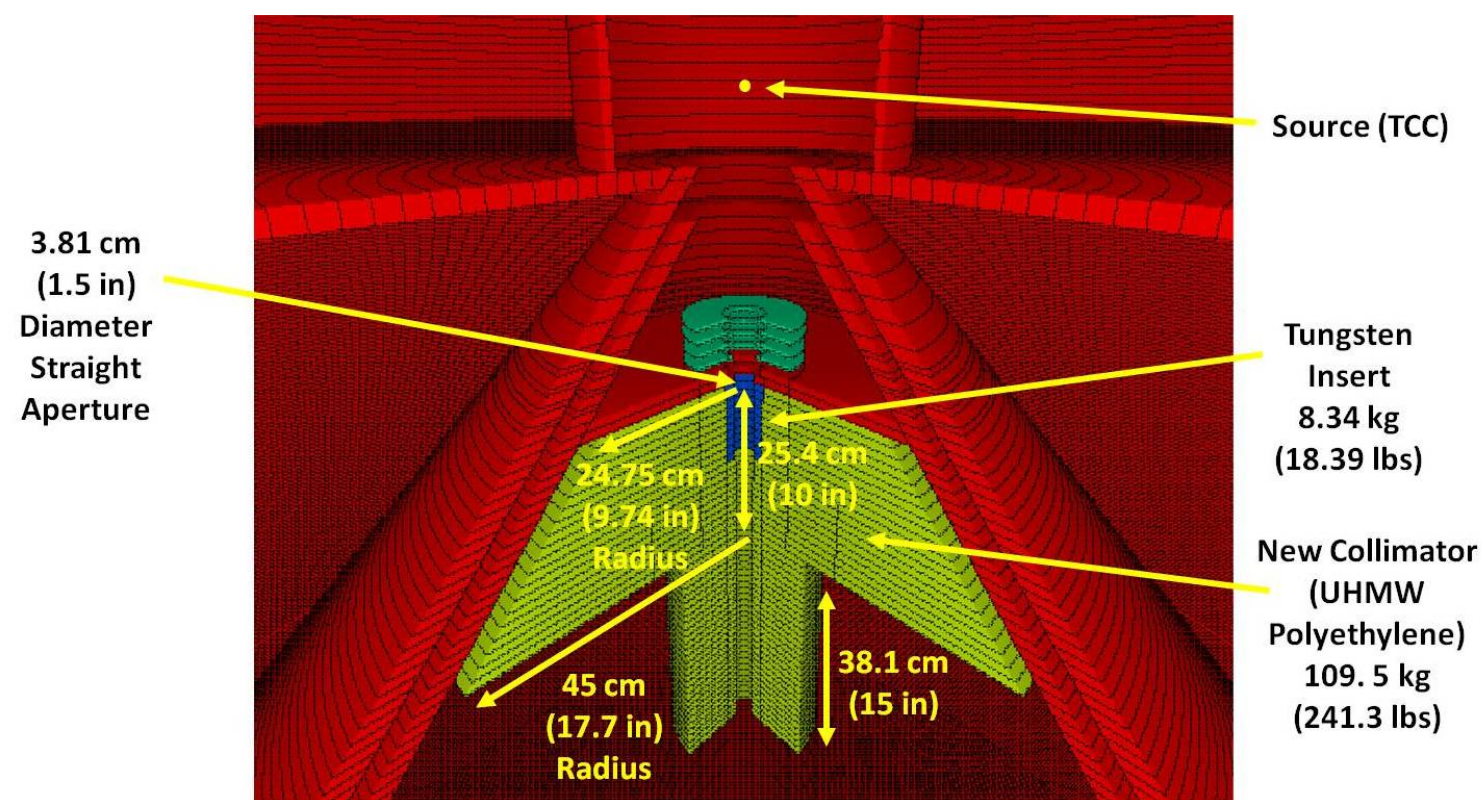

Figure 50. The new collimator design. The top radius is $24.75 \mathrm{~cm}(9.74 \mathrm{in})$ and the bottom radius is $45 \mathrm{~cm}$ (17.7 in). A cylinder of ultra-high molecular weight polyethylene (TIVAR 1000) [27] was added along the axis, giving it a mushroom appearance. The aperture along the Z-axis was $3.81 \mathrm{~cm}$ (1.5 in) in diameter and did not taper. The mass of the collimator was $109.5 \mathrm{~kg}(241.3 \mathrm{lbs})$, and the mass of the tungsten insert was $8.3 \mathrm{~kg}(18.4 \mathrm{lbs})$. Since the collimator had to be installed on the machine by hand by the center section, it was installed in sections due to its massive weight.

collimator had to be installed on the machine by hand by the center section, it was installed in sections due to its massive weight. 
The model with the old collimator (Figure 49) is shown in Figure 51 for the bottom nTOF (location " $D$ " in Figure 1). Of note is the additional, second scattering peak that occurs later in time, at about 460 ns.

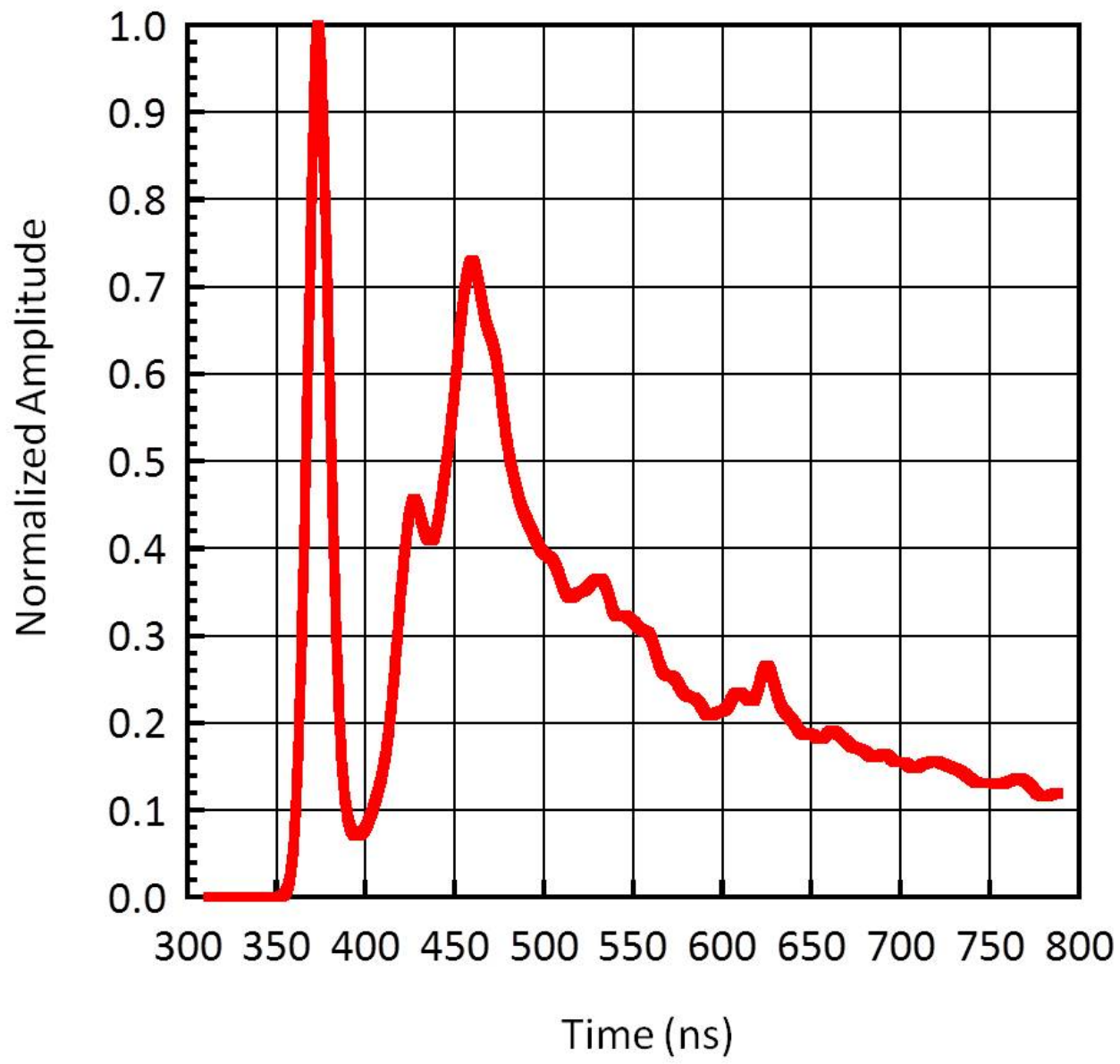

Figure 51. The model with the old collimator (Figure 49) for the bottom nTOF (location " $D$ " in Figure 1). Despite the fact that the addition of the old collimator improved the signal, there is still a second scattering peak which occurs later in time at about $460 \mathrm{~ns}$.

The model with the new collimator (Figure 50) is shown in Figure 52 for the bottom nTOF (location " $\mathrm{D}$ " in Figure 1). Note that the second scattering peak in Figure 51 goes away, leaving a very clean neutron signal. 


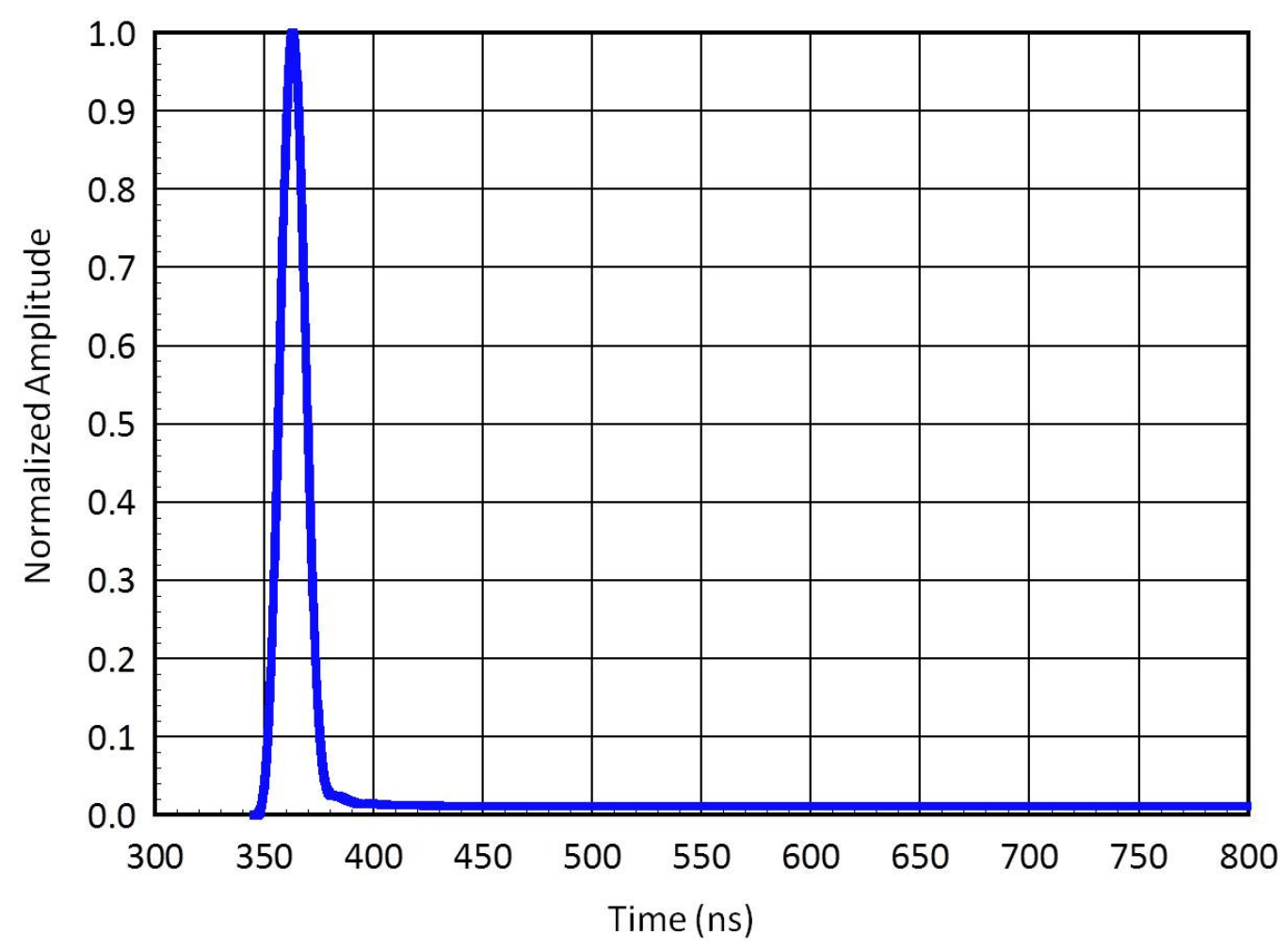

Figure 52. The model with the new collimator (Figure 50) for the bottom nTOF (location " $D$ " in Figure 1). Note that the second scattering peak shown in Figure 51 has gone away, leaving a very clean neutron signal.

Figure 53 shows the model of the old collimator (Figure 49) for the top nTOF (location " $\mathrm{C}$ " in Figure 1). Although the old collimator did improve the signal, there is still some scattering later in time, past the primary neutron peak. Figure 54 shows the model of the new collimator (Figure 50) for the top nTOF (location " $C$ " in Figure 1). Note that the scattering later in time is greatly reduced, showing that the additional mass of the new collimator was necessary to improve the neutron signal. 


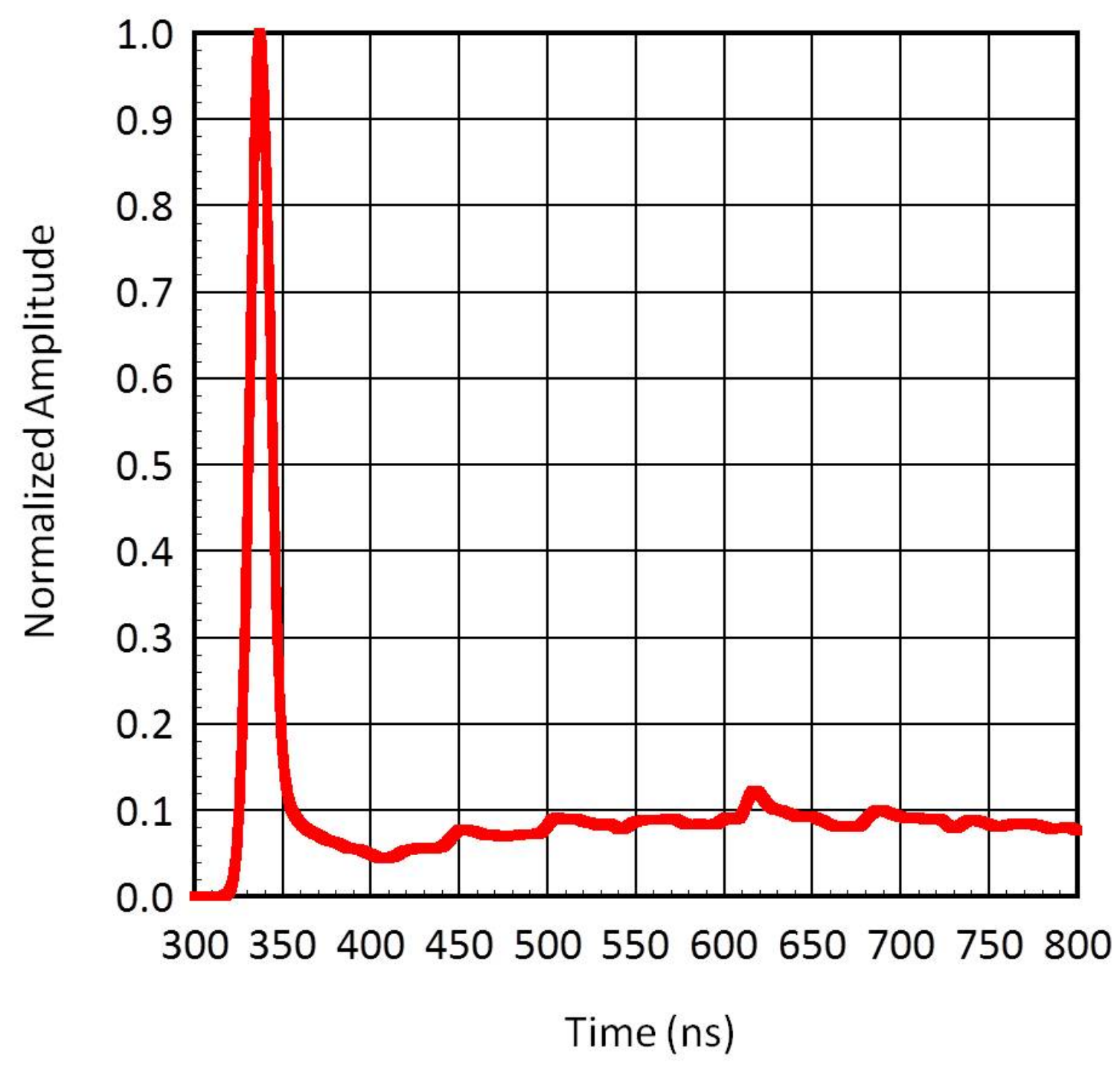

Figure 53. The model of the old collimator (Figure 49) for the top nTOF (location " $C$ " in Figure 1). Although the addition of the old collimator did improve the signal, there is still scattering later in time past the primary neutron peak. 


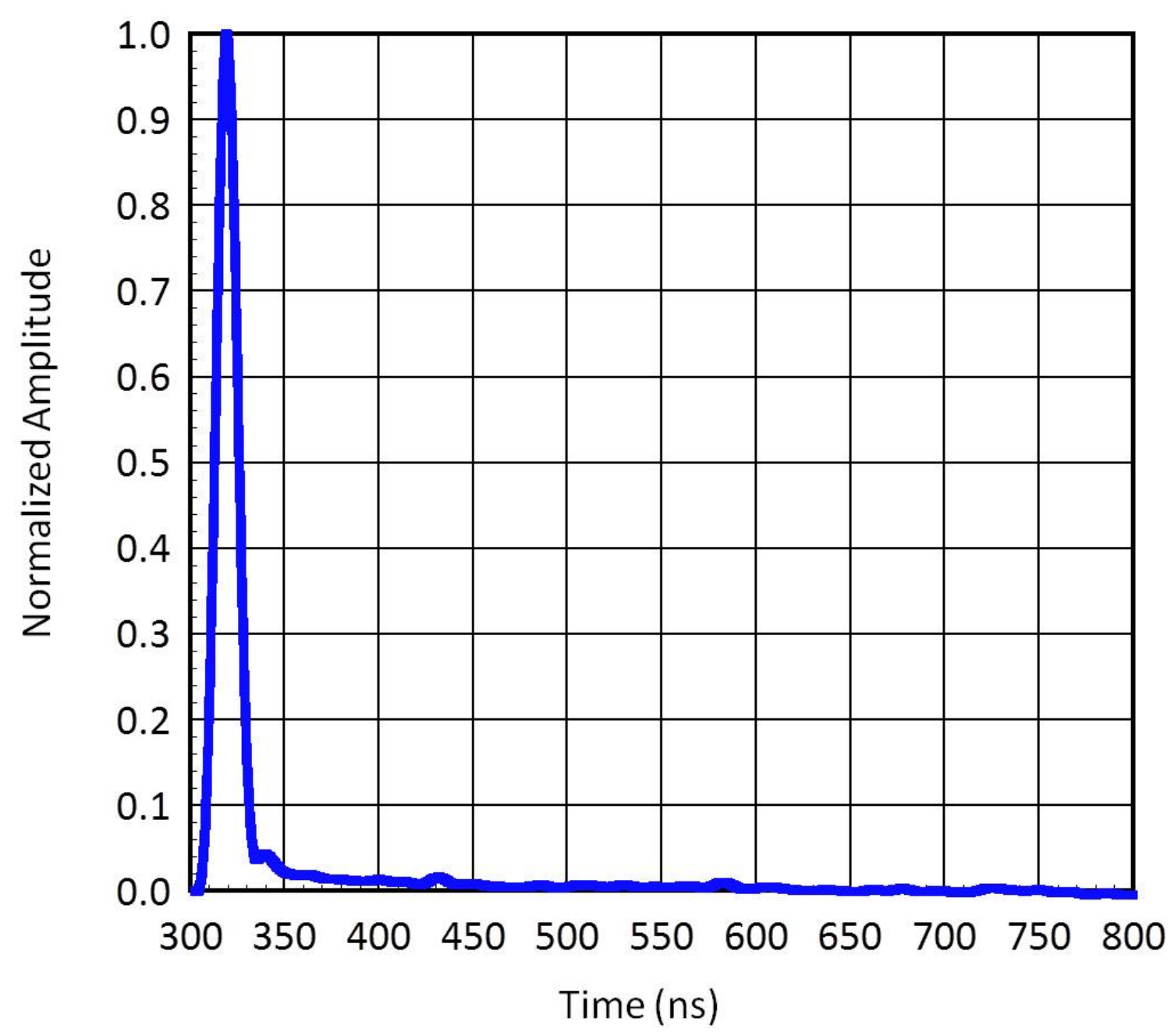

Figure 54. The model with the new collimator for the top nTOF (location " $\mathrm{C}$ " in Figure 1). Note that the scattering later in time is greatly reduced, showing that the additional mass of the new collimator was necessary to improve the neutron signal. 


\section{REFERENCES}

[1] Matzen, K., Phys. Plasmas 4, 1519 (1997).

[2] Spielman, R. B., et al., Proceedings of the 9th IEEE Pulse Power Conference, Albuquerque, NM, edited by R. White and K. Prestwich (Institute of Electrical and Electronics Engineers, New York, 1995), p. 396.

[3] J. Nuckolls et al., Nature 239, 139 (1972).

[4] T. J. Nash et al., Phys. Plasmas 6, 2023 (1999).

[5] J.F. Briesmeister (Ed). MCNP - A General Monte Carlo N-Particle Transport Code, Version 4C, LA-13709-M, Los Alamos National Laboratory, April 2000.

[6] S.A. Pozzi, E. Padovani, M. Marsequerra, Nucl. Instr. and Meth. A 513 (2003) 550558.

[7] J.T. Mihalczo, J.A. Mullens, J.K. Mattingly, T.E. Valentine, Nucl. Instr. and Meth. A 450 (1998) 531.

[8] S.A. Pozzi, J.K. Mattingly, J.T. Mihalczo, E. Padovani, "Validation of the MCNP-PoliMi Code for the Simulation of Nuclear Safeguards Experiments on Uranium and Plutonium 
Metal," Nuclear Mathematical and Computational Sciences: A Century in Review, A Century Anew, Gatlinburg, Tennessee, April 6 - 11, 2003, on CD-ROM, American Nuclear Society, LaGrange Park, IL., 2003.

[9] E. Padovani, S.A. Pozzi, MCNP-PoliMi ver.1.0 User's Manual, CESNEF-021125, Library of Nuclear Engineering Department, Politecnico di Milano, November 2002.

[10] Knoll, G.F., Radiation Detection and Measurement, John Wiley and Sons, NY., 1979.

[11] $1 \mathrm{MeVee}=$ amount of light produced by $1 \mathrm{MeV}$ deposited by a Compton scattered electron.

[12] Birks, J.B., The Theory and Practice of Scintillation Counting, Pergamon Press, NY., 1964.

[13] M. Marseguerra, E. Padovani, S.A. Pozzi, M.D. Ros, Nucl. Inst. and Meth. B 213 (2004) 289-293.

[14] N.R. Stanton, "A Monte Carlo Program for Calculating Neutron Detection Efficiencies in Plastic Scintillator, "Ohio State University Preprint COO-1545-92, Columbus, OH, USA, 1971. 
[15] J.B. Czirr, D.R. Nygren, C.D. Zafiratos, Nucl. Instr. and Meth. 31 (1964) $226-232$.

[16] V.V. Verbinski, W.R. Burrus, T.A. Love, W. Zobel and N.W. Hill, Nucl. Instr. and Meth. 65 (1968) $8-25$.

[17] S.A. Pozzi, J.A. Mullens, and J.T. Mihalczo, " Analysis of neutron and photon detection position for the calibration of plastic (BC-420) and liquid (BC-501) scintillators, “ Nucl. Inst. and Meth. A 524/1-3 pp. 92-101, 2004.

[18] D.B. Pelowitz (Ed), “ MCNPX User's Manual,“ Version 2.5.0, LA-CP-05-0369, Los Alamos National Laboratory, April 2005.

[19] Tektronix Corporation, Beaverton, OR 97077.

[20] J.S. Hendricks and T.E. Booth, " MCNP Variance Reduction Overview, " Radiation Transport Group X-6, LANL, 87544, Los Alamos, NM, 1985.

[21] Shultis, J.K., and Faw, R.E., " An MCNP Primer, " Dept. of Mechanical and Nuclear Engineering, Kansas State University, Manhattan, KS 66506, 2004.

[22] Savitsky, A., and Golay, M. J. E., "Smoothing and Differentiation of Data by Simplified Least Squares Procedures," Analytical Chemistry 36, (8): 1627 - 1639. 
[23] For more information, see "Savitzky-Golay smoothing filter" at http://en.wikeipedia.org

[24] For a summary of IAC facilities and contact information, see http://www.iac.isu.edu

[25] W.H. Press, S.A. Teukolsky, W.T. Vetterling, and B.P. Flannery, NUMERIAL RECIPES

in FORTRAN, The Art of Scientific Computing, $2^{\text {nd }}$ Ed., Cambridge University Press, 1992.

[26] J.R. Taylor, An Introduction to Error Analysis, $2^{\text {nd }}$ ed., University Science Books, Sausalito, CA, 1997.

[27] For a summary TIVAR 1000 properties and contact information, see http://www.quadrantplastics.com

[28] National Security Technologies, North Las Vegas Operations, North Las Vegas, Nevada.

[29] Personal Correspondence with Dr. Carlos Ruiz, PMTS, of Sandia National Laboratories.

[30] 1 shake $=10^{-08} \mathrm{sec}=10 \mathrm{~ns}$. 
[31] C.L. Ruiz, G.W. Cooper, S.A. Slutz, J.E. Bailey, G.A. Chandler, T.J. Nash, T.A. Mehlhorn, R.R. Leeper, D. Fehl, A.J. Nelson, J. Franklin, and L. Ziegler, Physical Review Letters, 93, 1, 2 July 2004.

[32] T.R. Boehly, D.L. Brown, R.S. Craxton, R.L. Keck, J.P. Knauer, J.H. Kelly, T.J. Kessler, S.A. Kumpan, S.J. Loucks, S.A. Letzring, F.J. Marshall, R.L. McCrory, S.F.B. Mose, W. Seka, J.M. Soures, and C.P. Verdon, "Initial performance results of the OMEGA laser system," Opt. Commun., vol. 133, pp. 495-506, 1997.

[33] E.I. Moses, "The National Ignition Facility: Status and plans for the experimental program," Fusion Sci. Technol., vol 44, pp. 11-18, 2003.

[34] Duderstadt and Moses, Inertial Confinement Fusion, John Wiley and Sons, Inc., Madison, WI, 1982.

[35] J. A. Frenje, D. T. Casey, C. K. Li, J. R. Rygg, F. H. Seguin, R. D. Petrasso, V. Yu. Glebov, D. D. Meyerhofer, T. C. Sangster, S. Hatchett, S. Haan, C. Cerjan, O. Landen, M. Moran, P. Song, D. C. Wilson, and R. J. Leeper, Rev. Sci. Instrum. 79, $10 \mathrm{E} 502$ (2008). 